\title{
SYSTEMS APPROACH AND QUANTITATIVE DECISION TOOLS FOR TECHNOLOGY SELECTION IN ENVIRONMENTALLY FRIENDLY DRILLING
}

\author{
A Dissertation \\ by \\ OK-YOUN YU \\ Submitted to the Office of Graduate Studies of \\ Texas A\&M University \\ in partial fulfillment of the requirements for the degree of \\ DOCTOR OF PHILOSOPHY
}

May 2009

Major Subject: Civil Engineering 


\title{
SYSTEMS APPROACH AND QUANTITATIVE DECISION TOOLS FOR TECHNOLOGY SELECTION IN ENVIRONMENTALLY FRIENDLY DRILLING
}

\author{
A Dissertation \\ by \\ OK-YOUN YU

\begin{abstract}
Submitted to the Office of Graduate Studies of
Texas A\&M University

in partial fulfillment of the requirements for the degree of

DOCTOR OF PHILOSOPHY
\end{abstract}

Approved by:

Chair of Committee, Jean-Louis Briaud

Committee Members, Roy Hann

Seth Guikema

David Burnett

Head of Department, David V. Rosowsky

May 2009

Major Subject: Civil Engineering 


\begin{abstract}
Systems Approach and Quantitative Decision Tools for Technology Selection in

Environmentally Friendly Drilling. (May 2009)

Ok-Youn Yu, B.S., KonKuk University; M.S., Texas A\&M University

Chair of Advisory Committee: Dr. Jean-Louis Briaud
\end{abstract}

One of the petroleum industry's goals is to reduce the environmental impact of oil and gas operations in environmentally sensitive areas. To achieve this, a number of Environmentally Friendly Drilling (EFD) technologies have been developed to varying degrees. For example, the use of an elevated platform as an alternative to the gravel pad is less intrusive and leads to a more environmentally friendly approach to drilling operations. Elevated drilling platforms will require the use of piles. Another alternative to the gravel pad is the use of composite mats. Since the demand of low impact technologies for drill site construction has rapidly increased, the parametric study for the feasibility of using pile foundations and composite mats is conducted in this research.

Even though a number of EFD technologies have already been developed to varying degrees, few have been integrated into a field demonstrable drilling system (i.e., combination of technologies) compatible with ecologically sensitive areas. In general, it is difficult to select the best combination of EFD technologies for a given site because there are many possible combinations and many different evaluation criteria. The proposed technology evaluation method is based on a systems analysis that can be used for integrating current and new EFD technologies into an optimal EFD system. An optimization scheme is suggested based on a combination of multi-attribute utility theory and exhaustively enumerating all possible technology combinations to provide a quantitative rationale and suggest the best set of systems according to a set of criteria, with the relative importance of the different criteria defined by the decision-maker. In 
this research, the sensitivity of the optimal solution to the weight factors and the effects of the uncertainty of input scores are also discussed using a case study.

An application of the proposed approach is described by conducting a case study in Green Lake at McFaddin, TX. The main purpose of this case study is to test the proposed technology evaluation protocol in a real site and then to refine the protocol. This research describes the results of the case study which provided a more logical and comprehensive approach that maximized the economic and environmental goals of both the landowner and the oil company leaseholder. 


\section{DEDICATION}

My father, In-Bok Yu, and mother, Sook-Ja Hyun, for their unconditional support, and My wife, Hei-Young, for her unconditional help and lovely nagging, and

My son, Jimin, and Daughter, Soomin, for their lovely smile 


\section{ACKNOWLEDGEMENTS}

To help me come this far, I received strong support and encouragement from my family. My parents financed me through my Ph.D. study and my wife, Hei-Young, had to give up many opportunities and quality of life. Big smile from my kids, Jimin and Soomin, helped me keep going forward. My accomplishment would have not been possible without their love.

I must express my sincere appreciation to my committee chair, Dr. Briaud, and my committee members, Dr. Guikema, Dr. Burnett, and Dr. Hann, for their guidance and support throughout the course of this research.

I also would like to thank the EFD subject matter experts for their assistance with this research, particularly Dr. Jerome Schubert, Ms. Carole Fleming, Dr. John Rogers, and Dr. Richard Haut. I also want to extend my gratitude to all other EFD project participants who shared their knowledge of drilling operations with me, and to $\mathrm{Mr}$. JongWha Chang for helping me find emission standards of various drilling technologies.

The information contained in this dissertation is part of the research project entitled "Field Testing of Environmentally Friendly Drilling Systems" sponsored by the U.S. Department of Energy and companies from the oil and gas industry. I would like to thank all of the sponsors. 


\section{TABLE OF CONTENTS}

Page

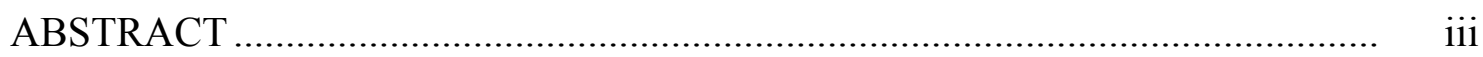

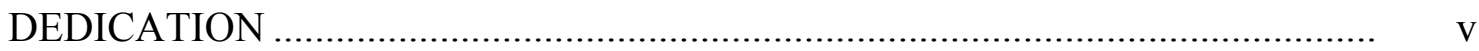

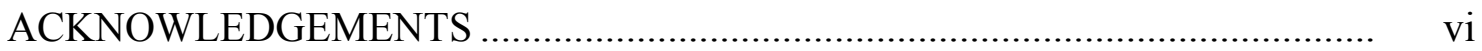

TABLE OF CONTENTS ....................................................................... vii

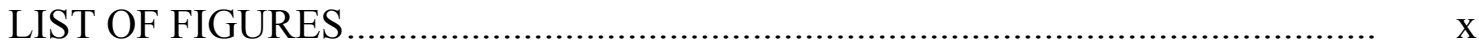

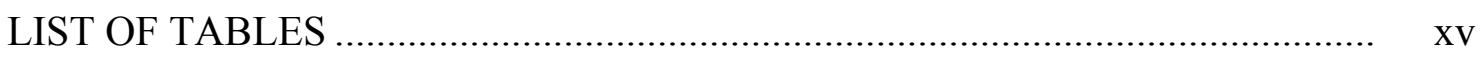

1. INTRODUCTION ..............................................................................

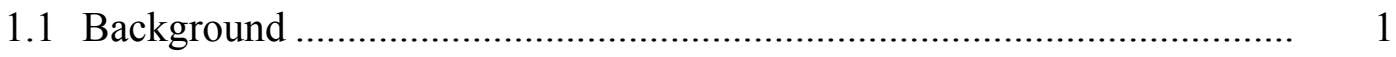

1.2 Problems .................................................................................. 2

1.3 Research Objectives ............................................................................ 3

2. METHODOLOGY ............................................................................. 4

2.1 Parametric Study of Foundations for Drill Sites ................................... 4

2.2 Development of a Systems Approach to Technology Evaluation............. 4

2.3 A Case Study with Pre-Specified Systems .......................................... 6

3. EXISTING KNOWLEDGE ...................................................................

3.1 Onshore Drilling Sequence ............................................................. 7

3.2 Pile Foundation Design ........................................................................ 7

3.2.1 Axial Pile Capacity ............................................................ 8

3.2.1.1 Driven Pile................................................................. 9

3.2.1.2. Bored Pile ................................................................ 11

3.2.2 Lateral Pile Capacity................................................................ 13

3.2.2.1 Free Head Case.............................................................. 14

3.2.2.2 Fixed Head Case.............................................................. 17

3.2.3 Pile Capacity Check................................................................. 19

3.2.3.1 Load and Resistance Factor Design (LRFD) Method........ 20

3.2.3.2 Working Stress Design (WSD) Method .......................... 21

3.3 Decision Analysis................................................................................ 22 
3.3.1 The Assumption of Utility Function ............................................... 23

3.3.2 Forms of the Utility Function.......................................................... 24

3.3.3 Sensitivity Analysis..................................................................... 25

4. PARAMETRIC STUDY OF FOUNDATIONS FOR DRILL SITES ................. 26

4.1 Foundation Options for Drill Sites ………………................................... 26

4.2 Pile Foundation Designs for Low Impact Onshore Platforms .................. 28

4.2.1 Description of the General Case .................................................... 29

4.2.1.1 Soil Conditions ............................................................... 30

4.2.1.2 Weight Distribution on Platform...................................... 31

4.2.1.3 Pile Capacity Check ........................................................... 32

4.2.1.4 Results Summary............................................................ 32

4.2.2 Description of an Elevated Platform with Rapid Rig ..................... 35

4.2.2.1 Soil Conditions ................................................................ 37

4.2.2.2 Weight Distribution on the Platform ................................. 37

4.2.2.3 LRFD Method ............................................................... 41

4.2.2.4 Results Summary............................................................ 42

4.2.3 Description of an Elevated Platform with Rapid Rig and a

Wind Turbine ....................................................................... 43

4.2.3.1 Soil Conditions .................................................................... 47

4.2.3.2 Weight Distribution on Platform ........................................ 47

4.2.3.3 LRFD Method ................................................................. 54

4.2.3.4 Results Summary......................................................... 55

4.2.4 Construction Strategies of Pile Foundation …………………......... 55

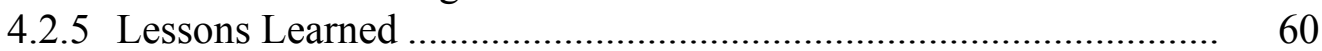

4.3 Feasibility of Using Composite Mat System in Drill Sites ........................ 61

4.3.1 Specification of DURA-BASE Composite Mat System................... 61

4.3.2 Finite Element Analysis for the Composite Mat System................. 61

4.3.3 Lessons Learned ....................................................................... 65

5. SYSTEMS APPROACH FOR TECHNOLOGY SELECTION ………............... 66

5.1 Research Methodology ....................................................................... 66

5.2 Identify the Main Subsystems, Subsets, and Technologies within Each Subset for the EFD Operation ......................................................... 67

5.3 Develop Attributes and Attribute Scales ..................................................... $\quad 70$

5.4 Assign Scores to All Technologies Using the Attribute Scales ................. 75

5.5 Calculate the Overall Attribute Score for Each Attribute .......................... 81

5.6 Develop a Utility Function for Each Attribute.......................................... 82

5.7 Combine Utility Values of Each Attribute into an Overall Utility ............ 84

5.8 Find the Best System............................................................................. 86 
5.9 Lessons Learned ......................................................................... 86

6. A CASE STUDY WITH PRE-SPECIFIED SYSTEMS ……….......................... 88

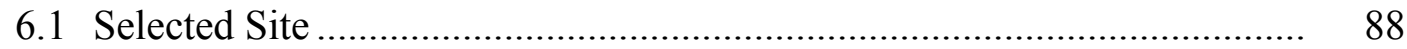

6.2 Description of the Pre-Specified Systems ................................................ 89

6.3 Calculate Overall Utility Values of the Pre-Specified Systems ................. $\quad 90$

6.4 Conduct a Sensitivity Analysis …………………............................... 100

6.4.1 Sensitivity Analysis for Weighting Factors of Each Attribute ........ 100

6.4.2 Sensitivity Analysis for Uncertainty of Overall Attribute Scores ... 114

6.5 Lessons Learned .............................................................................. 120

7. PROTOTYPE OF A WEB-BASED DECISION OPTIMIZATION TOOL ...... 122

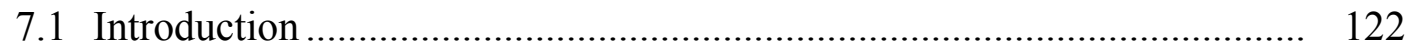

7.2 Input and Output Appearance .......................................................... 122

7.3 Evaluation of the Web-Based Application.............................................. 124

8. SUMMARY AND CONCLUSIONS........................................................ 126

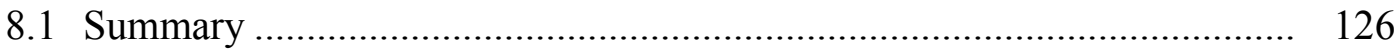

8.1.1 Parametric Study of Foundations for Drill Sites............................ 126

8.1.2 Development of a Systems Approach to Technology Evaluation ... 127

8.1.3 A Case Study with Pre-Specified Systems .................................... 128

8.1.4 Development of a Prototype of a Web-Based Application.............. 128

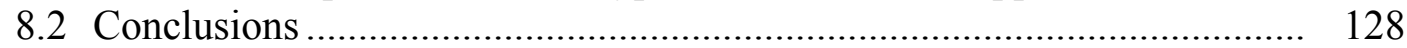

8.3 Future Tasks .................................................................................... 130

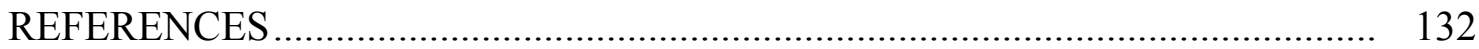

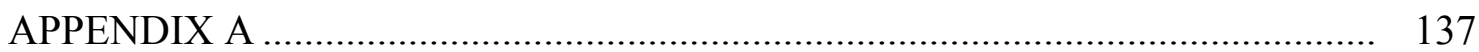

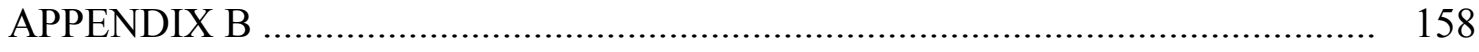

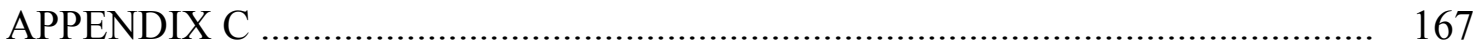

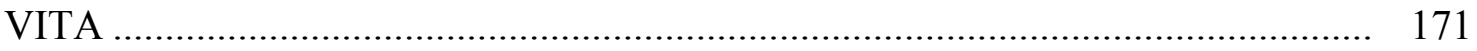




\section{LIST OF FIGURES}

FIGURE Page

3-1 Schematic drawing of an axially loaded pile ..................................... 8

3-2 Types of connections................................................................. 13

3-3 Free body diagram of pile down to zero-shear depth ........................... 15

4-1 Module dimension ..................................................................... 29

4-2 Plan view of modules .................................................................. 29

4-3 Cross section of the platform for one module in design ....................... $\quad 30$

4-4 Rapid Rig layout.............................................................................. 36

4-5 Three dimensional (3-D) layout of Rapid Rig .................................. 36

4-6 The applied load layout of Rapid Rig on the proposed platform .............. 38

4-7 Pile reaction force [kips], (using only four piles per module) ................. 38

4-8 Module deformation [ft], (using only four piles per module) .................. 39

4-9 Module deformed shape (using only four piles per module) .................. 39

4-10 Pile reaction force [kips], (using six piles for critical modules) .............. 40

4-11 Module deformation [ft], (using six piles for critical modules) ............... 40

4-12 Module deformed shape (using six piles for critical modules) ................ 41

4-13 Layout of Rapid Rig and the wind turbine ......................................... 46

4-14 Three dimensional (3-D) layout of Rapid Rig and the wind turbine......... 46

4-15 Applied load layout of Rapid Rig and the wind turbine........................ 47

4-16 Pile reaction force [kips] in operating condition ................................ 48 
FIGURE $\quad$ Page

4-17 Module deformation [ $\mathrm{ft}]$ in operating condition ................................ 49

4-18 Module deformed shape in operating condition................................. 49

4-19 Pile reaction force $[\mathrm{kips}]$ in extreme condition ............................... 50

4-20 Module deformation [ $\mathrm{ft}]$ in extreme condition................................. 50

4-21 Module deformed shape in extreme condition ................................. 51

4-22 Pile reaction force [kips] in operating condition (using six piles) ............ 51

4-23 Module deformation [ $\mathrm{ft}]$ in operating condition (using six piles) ............. 52

4-24 Module deformed shape in operating condition (using six piles) ............ 52

4-25 Pile reaction force [kips] in extreme condition (using six piles)............. 53

4-26 Module deformation [ft] in extreme condition (using six piles) ............... 53

4-27 Module deformed shape in extreme condition (using six piles) .............. 54

4-28 Assumed soil condition ............................................................... 56

4-29 Layout of Rapid Rig on the elevated platform ................................... 56

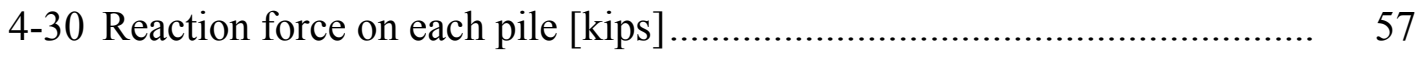

4-31 Numbers assigned to each pile ...................................................... 57

4-32 Actual mesh generated for this parametric analysis ............................ 62

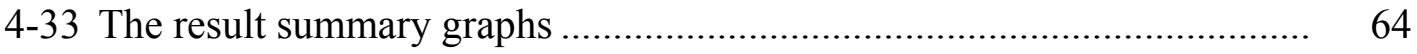

5-1 The structure of the EFD operation ................................................... 67

5-2 List of technologies within each subset........................................... 68

5-3 An example of the EFD technology selection..................................... 69

5-4 A typical layout of a conventional drilling site .................................. 75 
FIGURE $\quad$ Page

5-5 Cost estimation of gravel road ................................................... 76

5-6 Cost estimation of gravel pad ..................................................... 76

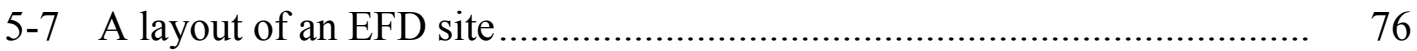

5-8 Cost estimation of Dura-Base Composite Mat................................... 77

5-9 Cost estimation of an elevated platform....................................... 77

5-10 Brief influence diagram of a drilling project.................................... 79

5-11 Selection procedure and constraints for the "Rig" subsystem ................ 80

5-12 An example of input scores ...................................................... 80

5-13 Overall attribute score for each attribute of a system.......................... 82

5-14 The single-attribute utility function curve for cost............................ 83

5-15 Single-attribute utility values of a system ..................................... 84

5-16 An example of the multi-attribute utility value of a system.................... 85

6-1 A satellite map of Green Lake at McFaddin, Texas.............................. 88

6-2 Basic assumptions and key influence variables ............................... 90

6-3 Influence diagram for the drilling site of the case study ....................... 91

6-4 Selection procedure for the "Rig" subsystem of the case study.............. 92

6-5 An example of input scores of the case study .................................. 92

6-6 The single-attribute utility function curves ...................................... 94

6-7 Overall utility score of the conventional drilling system ...................... 95

6-8 Overall utility score of the moderately improved drilling system ............ 96

6-9 Overall utility score of the EFD system in five years .......................... 96 
FIGURE $\quad$ Page

6-10 Total number of possible systems used in this cast study ..................... 97

6-11 Overall utility score of the best system ........................................ 98

6-12 Comparison of single-attribute utility scores and overall utility scores..... 98

6-13 Six systems suggested for Green Lake drilling site .......................... 103

6-14 Comparison of the single-attribute utility scores ............................. 104

6-15 Optimal utility scores of the suggested systems when $\mathrm{W}_{1}$ is varied ......... 106

6-16 Optimal utility scores of the suggested systems when $W_{2}$ is varied ......... 106

6-17 Optimal utility scores of the suggested systems when $W_{3}$ is varied ......... 107

6-18 Optimal utility scores of the suggested systems when $\mathrm{W}_{4}$ is varied ........ 107

6-19 Optimal utility scores of the suggested systems when $W_{5}$ is varied ......... 108

6-20 Optimal system selection as a function of $\mathrm{W}_{1}, \mathrm{~W}_{2}$, and $\mathrm{W}_{3} \ldots \ldots \ldots \ldots \ldots \ldots \ldots . . \ldots 109$

6-21 Optimal system selection as a function of $\mathrm{W}_{1}, \mathrm{~W}_{2}$, and $\mathrm{W}_{4} \ldots \ldots \ldots \ldots \ldots \ldots . . . \ldots 109$

6-22 Optimal system selection as a function of $\mathrm{W}_{1}, \mathrm{~W}_{2}$, and $\mathrm{W}_{5} \ldots \ldots \ldots \ldots \ldots \ldots \ldots . \ldots \ldots$

6-23 Optimal system selection as a function of $\mathrm{W}_{1}, \mathrm{~W}_{3}$, and $\mathrm{W}_{4} \ldots \ldots \ldots \ldots \ldots \ldots \ldots . \ldots \ldots$

6-24 Optimal system selection as a function of $\mathrm{W}_{1}, \mathrm{~W}_{3}$, and $\mathrm{W}_{5} \ldots \ldots \ldots \ldots \ldots \ldots . . . \ldots 111$

6-25 Optimal system selection as a function of $\mathrm{W}_{1}, \mathrm{~W}_{4}$, and $\mathrm{W}_{5} \ldots \ldots \ldots \ldots \ldots \ldots \ldots \ldots \ldots \ldots$

6-26 Optimal system selection as a function of $\mathrm{W}_{2}, \mathrm{~W}_{3}$, and $\mathrm{W}_{4} \ldots \ldots \ldots \ldots \ldots \ldots \ldots . . \ldots \ldots$

6-27 Optimal system selection as a function of $\mathrm{W}_{2}, \mathrm{~W}_{3}$, and $\mathrm{W}_{5} \ldots \ldots \ldots \ldots \ldots \ldots \ldots . . \ldots \ldots \ldots$

6-28 Optimal system selection as a function of $\mathrm{W}_{2}, \mathrm{~W}_{4}$, and $\mathrm{W}_{5} \ldots \ldots \ldots \ldots \ldots \ldots . . \ldots \ldots$

6-29 Optimal system selection as a function of $\mathrm{W}_{3}, \mathrm{~W}_{4}$, and $\mathrm{W}_{5} \ldots \ldots \ldots \ldots \ldots \ldots \ldots . . . \ldots 113$

6-30 Input values and variation of the overall attribute scores of SET $1 \ldots \ldots \ldots . .114$ 
FIGURE $\quad$ Page

6-31 Input values and variation of the overall attribute scores of SET $6 \ldots \ldots \ldots . .115$

6-32 Spider graph for SET 1 with 'Even' weight combination in Table 6-7 .... 116

6-33 Tornado diagram for SET 1 with 'Even’ weight combination................. 116

6-34 Spider graph for SET 1 with 'Base' weight combination in Table 6-7..... 117

6-35 Tornado diagram for SET 1 with 'Base' weight combination ................ 117

6-36 Spider graph for SET 6 with 'Even' weight combination in Table 6-7 ..... 118

6-37 Tornado diagram for SET 6 with 'Even’ weight combination................ 118

6-38 Spider graph for SET 6 with 'Base' weight combination in Table 6-7..... 119

6-39 Tornado diagram for SET 6 with 'Base' weight combination ................ 119

7-1 Evaluation page for 'Road Construction' subset................................ 122

7-2 Assigned weight scenarios ..................................................... 123

7-3 An example of the final result page ................................................ 123

7-4 Result of survey for the web-based decision optimization tool .............. 125 


\section{LIST OF TABLES}

TABLE Page

3-1 Design parameters for coarse grained soils (API RP2A-LRFD, 2003) .... 10

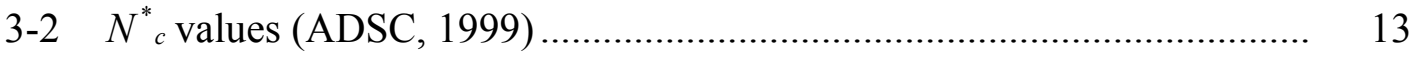

3-3 Load and resistance factors for driven piles (API RP2A-LRFD, 2003) ... 20

3-4 Recommended resistance factors for bored piles (ADSC, 1999)............. 20

3-5 Recommended factor of safety for driven piles (API RP2A-WSD, 2000) 22

3-6 Recommended factor of safety for bored piles (ADSC, 1999) ................ 22

4-1 Foundation options for a drilling site ............................................ 26

4-2 Soil conditions of pile capacity calculations ...................................... 31

4-3 Recommended size of driven piles in the general case (LRFD) .............. 33

4-4 Recommended size of driven piles in the general case (WSD) ............... 33

4-5 Recommended size of bored piles in the general case (LRFD) ............... 34

4-6 Recommended size of bored piles in the general case (WSD)................ 34

4-7 Load breakdown structure of Rapid Rig in operating condition ............... 35

4-8 Recommended size of driven piles in the Rapid Rig case (LRFD) .......... 42

4-9 Recommended size of bored piles in the Rapid Rig case (LRFD)........... 42

4-10 Specification of the wind turbine …................................................ 43

4-11 Load breakdown of Rapid Rig with the wind turbine in operating condition.

4-12 Load breakdown of Rapid Rig with the wind turbine in extreme condition. 
TABLE

4-13 Recommended size of driven piles in the Rapid Rig with wind turbine case

4-14 Recommended size of bored piles in the Rapid Rig with wind turbine case.

4-15 Optimal pile size and cost for each pile

4-16 Pile size for "using same pile" method

4-17 Pile size for "using two piles" method

60

4-18 Pile size for "categorized by reaction forces" method

4-19 Brief information about DURA-BASE Composite Mat System

4-20 $\rho=\mathrm{P}_{(\max )} / \mathrm{P}_{(\text {applied }) \text { values }}$

5-1 An example of air emission score calculation

5-2 Draft attribute scale for solid and liquid emission 72

5-3 Draft attribute scale for government perception. 73

5-4 Draft attribute scale for industry perception 73

5-5 Draft attribute scale for public perception 74

5-6 Draft attribute scale for safety value 74

5-7 Characteristic comparison of power generation technology 78

5-8 Benefits of reduced use of diesel to generate electricity in annual base rate

5-9 An example of assigned weight factor for each attribute

6-1 Pre-specified drilling systems

6-2 The base case weight factor for the each attribute 95

6-3 Weight combinations used in the sensitivity analysis 
TABLE Page

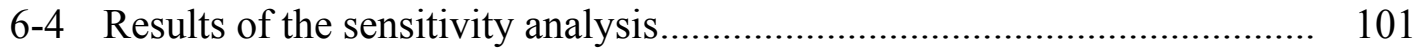

6-5 Range of the allowable weight factor for each attribute ...................... 102

6-6 Proportion of the optimal systems for this case study ........................... 103

6-7 Weight combinations used in the sensitivity analysis ....................... 114 


\section{INTRODUCTION}

\subsection{Background}

In the past 100 years the petroleum industry has provided important economic contributions and benefits to society through energy, wealth generation, and employment creation (Rogers et al. 2006). In the 21 st century the industry is being metamorphosed by the need to meet its social obligations and the need to improve economic performance by considering environmental impact of oil and gas operations. Recent studies conducted by the Department of the Interior show that almost $80 \%$ of federal lands containing more than 20 billion barrels of untapped oil is currently off limits to drilling. Only by utilizing low impact drilling practices can industry gain access to these environmentally sensitive areas.

Nowadays, the petroleum industry is endeavoring to develop such low impact or Environmentally Friendly Drilling (EFD) technologies to minimize the environmental impact during drilling operations. In environmentally sensitive areas, managing environmental impact will lead to greater access to large potential reserves in areas that are currently off-limit (Rogers et al. 2006). For example, directional drilling technology has allowed the industry to contact almost 60 times the volume of subsurface rock material that could be accessed in 1970 while occupying only one-third the surface area (Harrison 2005). Moreover, reducing the environmental footprint during drilling operations using a reusable Modular Platform and small mobile rig in the Arctic was demonstrated in 2003 by Anadarko and Noble's Subsidiary, Maurer technology Inc.. The objective was to drill in an ecologically sensitive area without disturbing the ground surface. The successful demonstration used a small mining rig to evaluate the potential of drilling for hydrates under the frozen tundra of the Alaska North Slope and showed the usefulness of an onshore platform to drill in environmentally sensitive areas (Kadaster and Millheim 2004).

This dissertation follows the style of the Journal of Geotechnical and Geoenvironmental Engineering. 


\subsection{Problems}

One of the petroleum industry's goals is to reduce the environmental impact of oil and gas operations in environmentally sensitive areas. To achieve this, a number of EFD technologies have been developed to varying degrees. For example, the use of an elevated platform as an alternative to the gravel pad for leveling and carrying capacity purposes is less intrusive and leads to a more environmentally friendly approach to oil and gas drilling operations. Elevated drilling platforms will require the use of piles. Another alternative to the gravel pad is the use of composite mats. As the demand of low impact technologies for drill site construction is rapidly increasing, parametric studies for the feasibility of using these technologies have become a more important part of the petroleum industry. In this research, the parametric study for the feasibility of using pile foundations and composite mats is conducted for various soil conditions and applied load areas.

Even though a number of EFD technologies and concepts have already been developed to varying degrees, few have been integrated into a field demonstrable drilling system (i.e., combination of technologies) compatible with ecologically sensitive or offlimits areas. Such sensitive areas include wetlands of the Gulf Coast and federal lands in the Western U.S. In general, it is difficult to select the best combination of EFD technologies for a given site because there are many possible combinations and many different and perhaps competing evaluation criteria. How to logically measure and select the best available EFD system for a specific site is fully described in this research. 


\subsection{Research Objectives}

The key objectives of this research are to:

1. Help the petroleum industry engineers to get a basic idea about environmentally friendly foundation designs of a rig or an elevated platform for various weights and soil conditions in environmentally sensitive areas (e.g., desert environments and wetland applications). In order to encourage petroleum industry people to use environmentally friendly foundations such as elevated platforms and composite mat systems more often for their drilling sites instead of using gravel pads, it is an essential task in this research.

2. Develop a technology evaluation protocol based on a systems analysis to synergistically incorporate a number of current and emerging EFD technologies into a single and clean drilling system with limited environmental impact and then to suggest a small number of systems that should be particularly attractive for a given site. This decision-analytic model will help decision-makers select an optimal drilling system for a given site to minimize environmental impact and maximize profit at that specific site.

3. Develop a prototype of a web-based decision optimization tool to help decisionmakers easily follow the proposed technology evaluation procedure and then select an optimal drilling system for a specific site. The web-based application can also help to manage used input parameters permanently if a central repository is maintained regularly so that decision-makers or drilling operators can easily retrieve a previously designed well model for their future operations in different ecosystems. 


\section{METHODOLOGY}

\subsection{Parametric Study of Foundations for Drill Sites}

Three different types of foundations for drill sites are considered in this research.

1. Two different types of pile foundations (i.e., driven pile and bored pile): elevated platforms will require the use of piles. About one thousand different cases of pile capacity calculations are conducted depending on various soil types, pile types, and design methods. The results of these calculations are organized into a series of tables for the petroleum industry engineer to choose an appropriate pile size for a given condition without performing an extensive pile design analysis. The optimal pile selection procedure is also described in this research.

2. Dura-Base Composite Mat: feasibility study of using the Dura-Base Composite Mat System for the drill site construction is demonstrated with various applied load areas from 6 inches to 10 feets in diameter and soil types.

\subsection{Development of a Systems Approach to Technology Evaluation}

The information contained in this research is part of the research project entitled "Field Testing of Environmentally Friendly Drilling Systems" sponsored by the U.S. Department of Energy and companies from the oil and gas industry. The main purpose of this project is to integrate current and new EFD technologies into a viable drilling system compatible with environmentally sensitive areas and finally to suggest a small number of systems $(1 \sim 5)$ that should be particularly attractive for a given site. The proposed method is based on a systems analysis that can be used for integrating current and new EFD technologies into an optimal EFD system. The system draws upon a large number of technologies (more than 100) identified by a government-industry joint venture studying low impact operations in sensitive ecological areas. In order to provide flexibility to the user, a small number of systems $(1 \sim 5)$ are proposed for a given site, instead of a single best system. An optimization scheme is suggested based on a 
combination of multi-attribute utility theory and exhaustively enumerating all possible technology combinations (i.e., exhaustive search optimization) to provide a quantitative rationale and suggest the best set of systems according to a set of criteria, with the relative importance of the different criteria defined by the decision-maker.

Since an optimal system for a specific site would be based on subjectively assessed data, there can be considerable uncertainty about the input parameters used. Therefore, even if finding the optimal system is valuable to the decision-makers, they also would like to know how robust the decision is to changes in the input parameters such as the attribute scales, weight factors for attributes, risk-attitude (i.e., risk-neutral, risk-averse, and risk-seeking), and single-attribute utility functions assessed by different individuals (Guikema and Milke 2003). In this research, a sensitivity analysis is conducted using a case study to address this problem.

The methodology described in this research is designed to help decision-makers select an optimal drilling system for a given site in order to minimize environmental impact and maximize profit at that specific site. The technology evaluation protocol can be refined based on EFD experts' inputs and feedbacks if necessary. Further interaction with appropriate experts would be valuable in revising this evaluation protocol. The overall procedure is briefly illustrated as follows:

Step 1: Identify the main subsystems, subsets, and technologies within each subset for the EFD operations.

Step 2: Define attributes and develop attribute scales to evaluate technologies.

Step 3: Assign scores to all technologies using the attribute scales.

Step 4: For each attribute, calculate the overall attribute score of a system by adding the technology scores or selecting the minimum technology score.

Step 5: For each attribute and in order to homogenize the scores, develop a "utility function $\left(\mathrm{u}_{\mathrm{i}}\right)$ " to convert the overall dimensional score of a system (e.g., \$, acres, and grades) into a non-dimensional utility value (between 0 and 1) of the system that reflects the decision-maker(s) value.

Step 6: Decide on a weight factor $\left(\mathrm{k}_{\mathrm{i}}\right)$ for each attribute $\left(\mathrm{i}^{\mathrm{th}}\right)$. 
Step 7: Calculate the overall score of the system as " $\sum \mathrm{k}_{\mathrm{i}} \mathrm{u}_{\mathrm{i}}$ " (multi-attribute utility function).

Step 8: Use optimization technique to evaluate all possible systems and to find the best system for a specific site. Once all possible systems have been evaluated, the system with the highest overall score is the best system.

Step 9: Conduct a sensitivity analysis to examine the impacts of possible changes in the attribute scores, weight factors, and utility functions on the optimal system.

Step 10: Suggest a small number of systems that should be attractive for a given site.

\subsection{A Case Study with Pre-Specified Systems}

An application of the proposed approach is described by conducting a case study in Green Lake at McFaddin, TX; some of the difficulties in using this approach in practice are also discussed. The main purpose of this case study is to test the proposed technology evaluation protocol in a real site and then to refine the protocol. Three different systems are pre-specified by an EFD expert in order to identify possible drilling technologies for Green Lake drilling site: (1) conventional drilling; (2) moderately improved drilling; and (3) EFD in five years. First, all technologies selected in these three systems are evaluated with respect to the nine attributes. Second, these three systems' overall scores are evaluated by the proposed technology evaluation protocol. Third, use optimization technique to evaluate all possible systems and to find the best system for Green Lake drilling site. The best system is the system with the highest overall score among all possible systems. After that, a sensitivity analysis is conducted to examine the impacts of possible changes in the attribute scores and weight factors on the optimal system. Finally, a small number of systems (1 5) that should be attractive for the site are suggested.

The results of the case study which provided a more logical and comprehensive approach that maximized the economic and environmental goals of both the landowner and the oil company leaseholder are described in this research. 


\section{EXISTING KNOWLEDGE}

\subsection{Onshore Drilling Sequence}

According to Dyke (1997), the standard drilling operation procedure is briefly illustrated as follows:

Step 1: Receive initial well planning information including Surface Hole Location (SHL) with Bottom Hole Location (BHL) if applicable.

Step 2: Confirm lease issues including surface ownership.

Step 3: Check the site specific state permit requirements.

Step 4: Check the topographical/ cultural requirements.

Step 5: Confirm operational parameters including mud system and disposal options (onsite vs. offsite).

Step 6: Construct access road.

Step 7: Construct pad (site preparation) including mud reserve pits if applicable.

Step 8: Place a rig and other required components.

Step 9: Drill the hole.

\subsection{Pile Foundation Design}

Use of a raised platform in environmentally sensitive areas will require the use of piles to support the elevated platform instead of gravel pads as used in a conventional platform. Piles are used to transfer the load from the structures on/above the ground surface to the underlying soil mass. The axially transferred loads are resisted by the friction between the pile and the surrounding soil as well as the end bearing resistance at the bottom of the pile. It is critical in pile designs to estimate the proper axial capacity of the pile depending on the pile and soil types. In addition, the lateral capacity of the pile also should be checked since most piles must resist the horizontal component of the applied loads. In other words, the designed pile should meet not only the axial capacity criterion but also the lateral capacity criterion. The estimated capacities of piles are 
checked against the applied loads according to a design method, such as the Load and Resistance Factor Design (LRFD) and the Working Stress Design (WSD).

\subsubsection{Axial Pile Capacity}

The ultimate capacity of the pipe piles is obtained by adding the outside skin friction and the end bearing resistance. The end bearing resistance assumes that the bottom of the pile is closed or that the open ended pipe pile would plug during static loading. The ultimate axial bearing capacity of a pile (Figure 3-1) can be expressed as the sum of the skin friction and end bearing resistances in Eq. (3-1):

$$
Q_{u}=Q_{f}+Q_{p}=\sum f_{i} \times A_{s i}+q \times A_{p}
$$

where, $Q_{u}=$ ultimate bearing capacity $(\mathrm{kN}, \mathrm{lbs})$,

$Q_{f}=$ skin friction resistance $(\mathrm{kN}, \mathrm{lbs})$

$Q_{p}=$ total end bearing $(\mathrm{kN}, \mathrm{lbs})$,

$f_{i}=$ unit skin friction capacity in $\mathrm{i}^{\text {th }}$ layer $\left(\mathrm{kPa}, \mathrm{lb} / \mathrm{ft}^{2}\right)$

$A_{s i}=$ side surface area of pile in $\mathrm{i}^{\text {th }}$ layer $\left(\mathrm{m}^{2}, \mathrm{ft}^{2}\right)$,

$A_{p}=$ gross end area of pile $\left(\mathrm{m}^{2}, \mathrm{ft}^{2}\right)$

$q=$ unit end bearing capacity $\left(\mathrm{kPa}, \mathrm{lb} / \mathrm{ft}^{2}\right)$

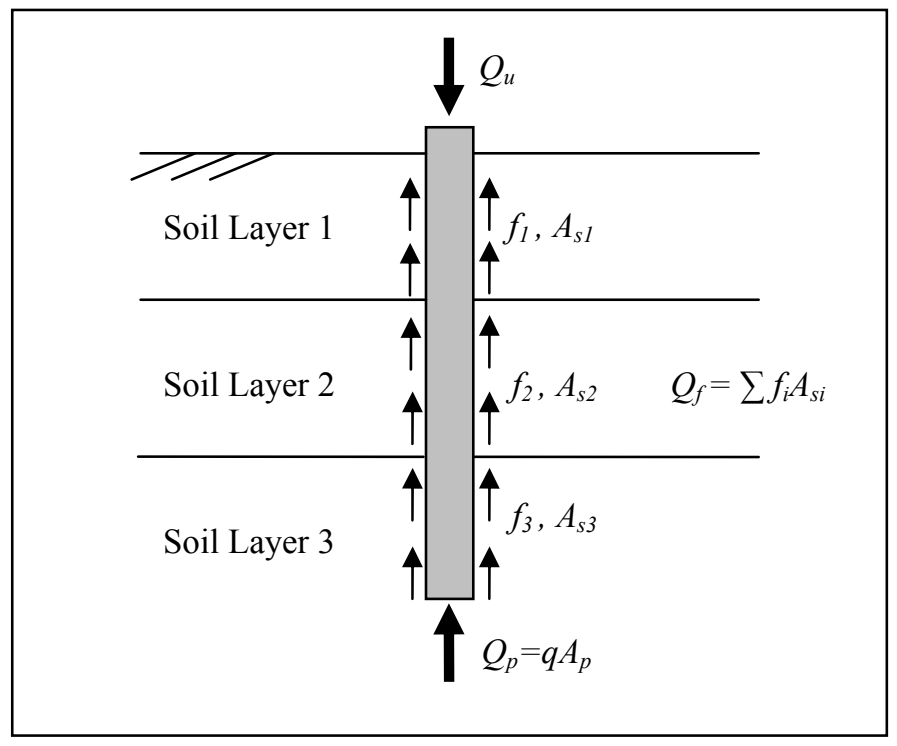

Figure 3-1. Schematic drawing of an axially loaded pile 
The skin friction and end bearing resistances are calculated in different ways depending on the pile type such as driven piles or bored piles. The type of underlying soil (i.e., fine grained or coarse grained soil) also affects the calculation method. The API RP2A-LRFD (2003), API RP2A-WSD (2000), and the ADSC (1999) are referred to the calculation procedures for the unit skin friction, $f_{\mathrm{i}}$, and the end bearing resistance, $q$, of driven piles and bored piles.

\subsubsection{Driven Pile}

The unit skin friction is the shear stress between the pile and soil at failure. According to the API RP2A-LRFD (2003) and API RP2A-WSD (2000), the unit skin friction of a driven pile in coarse grained soils can be calculated by Eq. (3-2):

$$
f=K \times p_{0}{ }^{\prime} \times \tan \delta
$$

where, $K=$ dimensionless coefficient of lateral earth pressure

$p_{0}{ }^{\prime}=$ effective overburden pressure at the point in question $\left(\mathrm{kPa}, \mathrm{lb} / \mathrm{ft}^{2}\right)$

$\delta=$ friction angle between the soil and pile wall

For open-ended pipe piles driven unplugged, it is usually suggested to assume $K$ as 0.8 for both tension and compression loadings while values of $K$ for full displacement piles (plugged or closed end) may be assumed to be 1.0. The friction angle of a soil, $\varphi$, corresponds to the friction coefficient $\mu_{1}$ of a soil-soil interface through: $\mu_{1}=\tan \varphi$. The angle $\delta$ is the friction angle which corresponds to the friction coefficient $\mu_{2}$ of the soilpile interface through $\mu_{2}=\tan \delta$. The unit end bearing of a driven pile in coarse grained soils can be computed by Eq. (3-3):

$$
q=p_{0}{ }^{\prime} \times N_{q}
$$

where, $N_{q}=$ dimensionless bearing capacity factor. Recommended values of $N_{q}$ are tabulated in Table 3-1. 
Table 3-1. Design parameters for coarse grained soils (API RP2A-LRFD, 2003)

\begin{tabular}{|l|l|c|c|c|c|}
\hline \multicolumn{1}{|c|}{ Density } & \multicolumn{1}{|c|}{$\begin{array}{c}\text { Soil } \\
\text { Description }\end{array}$} & $\begin{array}{c}\text { Friction } \\
\text { Angle, } \\
\delta(\mathrm{deg})\end{array}$ & $\begin{array}{c}\text { Limiting Skin } \\
\text { Friction } \\
\mathrm{kPa}\left(\mathrm{kips} / \mathrm{ft}^{2}\right)\end{array}$ & $N_{q}$ & $\begin{array}{c}\text { Limiting Unit } \\
\text { End Bearing } \\
\mathrm{MPa}\left(\mathrm{kips} / \mathrm{ft}^{2}\right)\end{array}$ \\
\hline $\begin{array}{l}\text { Very Loose } \\
\text { Loose } \\
\text { Medium }\end{array}$ & $\begin{array}{l}\text { Sand } \\
\text { Sand-Silt } \\
\text { Silt }\end{array}$ & 15 & $47.8(1.0)$ & 8 & $1.9(40)$ \\
\hline $\begin{array}{l}\text { Loose } \\
\text { Medium } \\
\text { Dense }\end{array}$ & $\begin{array}{l}\text { Sand } \\
\text { Sand-Silt } \\
\text { Silt }\end{array}$ & 20 & $67.0(1.4)$ & 12 & $2.9(60)$ \\
\hline $\begin{array}{l}\text { Medium } \\
\text { Dense }\end{array}$ & $\begin{array}{l}\text { Sand } \\
\text { Sand-Silt }\end{array}$ & 25 & $81.3(1.7)$ & 20 & $4.8(100)$ \\
\hline $\begin{array}{l}\text { Dense } \\
\text { Very Dense }\end{array}$ & $\begin{array}{l}\text { Sand } \\
\text { Sand-Silt }\end{array}$ & 30 & $95.7(2.0)$ & 40 & $9.6(200)$ \\
\hline $\begin{array}{l}\text { Dense } \\
\text { Very Dense }\end{array}$ & $\begin{array}{l}\text { Gravel } \\
\text { Sand }\end{array}$ & 35 & $114.8(2.4)$ & 50 & $12.0(250)$ \\
\hline
\end{tabular}

According to the API RP2A-LRFD (2003) and WSD (2000), the unit skin friction of a driven pile in fine grained soils can be calculated by Eq. (3-4):

$$
f=\alpha_{1} \times s_{u}
$$

where, $\alpha_{1}=$ dimensionless adhesion factor

$$
s_{u}=\text { undrained shear strength of the soil }\left(\mathrm{kPa}, \mathrm{lb} / \mathrm{ft}^{2}\right)
$$

The factor, $\alpha_{1}$ is an empirical adhesive factor for reduction of the average undrained shear strength. The $\alpha_{1}$ value can be calculated by Eq. (3-5) with the constraint that $\alpha_{1} \leq 1$.

$$
\begin{array}{ll}
\alpha_{1}=0.5 \times \psi^{-0.5} & (\psi \leq 1.0) \\
\alpha_{1}=0.5 \times \psi^{-0.25} & (\psi>1.0)
\end{array}
$$

where, $\psi=s_{u} / p_{0}{ }^{\prime}$

The shaft friction acts on both the inside and outside of the pile. The total shaft resistance is the sum of the external friction and the internal shaft friction if the internal shaft friction is less than the end bearing capacity.

The unit end bearing a driven pile in fine grained soils can be computed by Eq. (3-6):

$$
q=9 \times s_{u}
$$

where, $s_{u}=$ undrained shear strength $\left(\mathrm{kPa}, \mathrm{lb} / \mathrm{ft}^{2}\right)$ 
In fine grained soils, the capacity of piles follows an undrained analysis using $s_{u}$. The reason is that a fine grained soil does not have time to drain during the loading and this corresponds to the time where the fine grained soil is the weakest. Indeed right after the loading the pore pressures are high and the effective stress is low while in the long term the pore pressures generated by the loading dissipate, the effective stress increases and so does the shear strength of the fine grained soil. In coarse grained soils, the capacity of piles follows a drained analysis because a coarse grained soil has time to drain during loading.

\subsubsection{Bored Pile}

According to the ADSC (1999), the unit skin friction of a bored pile in coarse grained soils can be calculated by Eq. (3-7):

$$
f=\beta \times p_{0}^{\prime}
$$

where, $\beta=$ dimensionless correlation factor

Suggested values of $\beta$ for granular soils classified as sand can be obtained by Eq. (3-8) if $N_{S P T} \geq 15$ blows per $0.3 \mathrm{~m}$ :

$$
\beta=1.5-0.245 \times z(m)^{0.5}, \quad(0.25 \leq \beta \leq 1.20)
$$

where, $\mathrm{z}=$ depth below the ground surface in meter

If $N_{S P T}<15$ blows per $0.3 \mathrm{~m}, \beta$ value can be computed by Eq. (3-9):

$$
\beta=\left(N_{S P T} / 15\right)\left[1.5-0.245 \times z(m)^{0.5}\right], \quad(0.25 \leq \beta \leq 1.20)
$$

The unit end bearing of a bored pile in coarse grained soils can be computed by Eq. (3$10)$ :

$$
q(\text { tsf })=0.60 \times N_{S P T}
$$

where, $N_{S P T}=$ uncorrected SPT blow count (blows/ft)

The Standard Penetration Test (SPT) is a geotechnical field test. It is performed at the bottom of a borehole which is about 4 inches in diameter. The SPT consists of driving a standard sampler about 2.5 inches in diameter called the split spoon sampler starting at the bottom of an open borehole while using a standard $140 \mathrm{lbs}$ hammer. This 
hammer is raised 30 inches above the anvil and dropped freely for each blow. The number of blows required to drive the sampler one foot into the soil is recorded as the blow count $\mathrm{N}$ (bpf). The $\mathrm{N}$ values are obtained every 5 to 10 feet with depth and a blow count profile is generated.

According to the ADSC (1999), the unit skin friction of a bored pile in fine grained soils can be calculated by Eq. (3-11):

$$
f=\alpha_{2} \times s_{u}
$$

where, $\alpha_{2}=$ shear strength reduction factor

$=0$ between the ground surface and a depth of $1.5 \mathrm{~m}(5 \mathrm{ft})$

$=0$ for a distance of $B_{b}$ above the base

$=0.55$ for $s_{u} / P_{a} \leq 1.5$

$=0.55-0.1\left(s_{u} / P_{a}-1.5\right)$ for $1.5 \leq s_{u} / P_{a} \leq 2.5$

$B_{b}=$ diameter on the base of the bored pile $(\mathrm{m}, \mathrm{ft})$

$P_{a}=$ atmospheric pressure $\left(101 \mathrm{kPa}\right.$ or $\left.2116 \mathrm{lb} / \mathrm{ft}^{2}\right)$

$s_{u}=$ undrained shear strength of the soil $\left(\mathrm{kPa}, \mathrm{lb} / \mathrm{ft}^{2}\right)$

The $\alpha_{2}$ values are developed from measured data on full-scale load tests and depend on the undrained shear strength, $s_{u}$. If the fine grained soil has a value of $s_{u} \geq 96 \mathrm{kPa}$ $\left(2000 \mathrm{lb} / \mathrm{ft}^{2}\right)$, the unit end bearing of a bored pile in fine grained soils can be computed by Eq. (3-12):

$$
q=9 \times s_{u}
$$

However, if the embedded pile length $\left(L_{p}\right)$ is less than three times the diameter of the base of the bored pile $\left(3 B_{b}\right)$, then the unit end bearing capacity $(q)$ should be reduced as follows:

$$
q=0.667\left[1+0.1667\left(L_{p} / B_{b}\right)\right] N^{*} \times s_{u}
$$

where, $L_{p}=$ embedded pile length $(\mathrm{m}, \mathrm{ft})$

$B_{b}=$ diameter on the base of the bored pile $(\mathrm{m}, \mathrm{ft})$

$N^{*}{ }_{c}=$ modified bearing capacity factor

Recommended values of $N^{*}{ }_{c}$ are tabulated in Table 3-2. 
Table 3-2. $N^{*}{ }_{c}$ values (ADSC, 1999)

\begin{tabular}{|c|c|}
\hline$s_{u}$ & $N^{*}{ }_{c}$ \\
\hline $24 \mathrm{kPa}\left(500 \mathrm{lb} / \mathrm{ft}^{2}\right)$ & 6.5 \\
\hline $48 \mathrm{kPa}\left(1000 \mathrm{lb} / \mathrm{ft}^{2}\right)$ & 8.0 \\
\hline $96 \mathrm{kPa}\left(2000 \mathrm{lb} / \mathrm{ft}^{2}\right)$ & 8.7 \\
\hline $192 \mathrm{kPa}\left(4000 \mathrm{lb} / \mathrm{ft}^{2}\right)$ & 8.9 \\
\hline
\end{tabular}

\subsubsection{Lateral Pile Capacity}

Piles are often subjected to relatively large horizontal loads and overturning moment due to wind loads, seismic loads, etc. In this case, the lateral pile capacity should be checked for two criteria. The piles should have enough lateral soil bearing capacity to resist against the horizontal loads and the horizontal deflection of the pile should be within an allowable limit. The methods for performing lateral capacity analyses depend on the type of connection between the pile and the structure. If the pile is connected to the structure in such a way that the top of the pile may freely move laterally and rotate (Figure 3-2 a), it may be assumed to be a free head condition. If the top of the pile may move laterally but is not allowed to rotate (Figure 3-2 b), it may be assumed to be a fixed head condition.

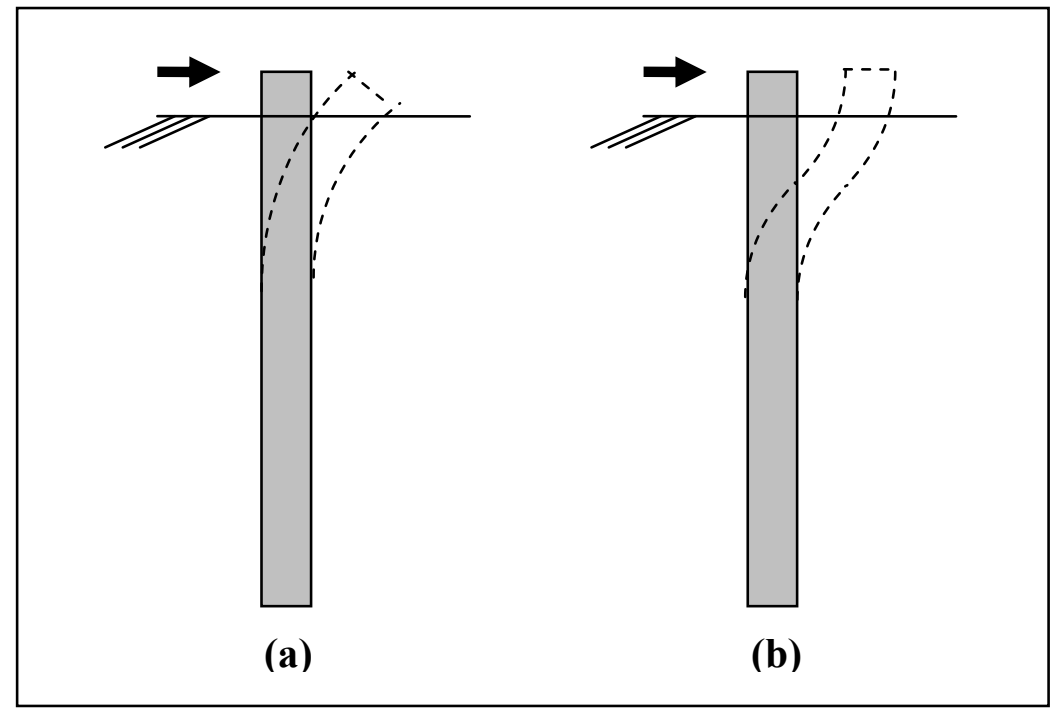

Figure 3-2. Types of connections. (a) free head, and (b) fixed head 


\subsubsection{Free Head Case}

The spring constant, $K_{s}$, is the ratio of the lateral resistance of the soil per unit length of a pile to the lateral displacement of the pile. It can be obtained by Eq. (3-14) (Briaud 1997):

$$
K_{s}=2.3 E_{0}
$$

$E_{0}$ is the first load pressuremeter (PMT) modulus. The pressuremeter is a geotechnical field test. It consist of drilling a 3 inch borehole, removing the drilling tool, lowering a cylindrical probe about $2.5 \mathrm{ft}$ in length and 3 inch in diameter, and expanding that probe laterally against the borehole walls while recording the volume of the probe and the pressure exerted on the soil. This gives an in situ stress strain curve from which a soil modulus $\left(E_{0}\right)$ and a horizontal limit pressure $\left(P_{L}\right)$ are obtained. $E_{0}$ can be obtained by using the following correlations if PMT tests are not available:

$$
\begin{aligned}
E_{0}(\mathrm{kPa}) & =383 N_{S P T}(\text { blow } / 30 \mathrm{~cm}), \text { or } E_{0}(\mathrm{tsf})=4 N_{S P T}(\text { blow } / \mathrm{ft})(\text { Briaud 1992) } \\
& =\text { average pressuremeter modulus }(\mathrm{kPa}, \mathrm{tsf})
\end{aligned}
$$

where, $N_{\mathrm{SPT}}=$ blow count in Standard Penetration Test

The factor 2.3 is determined empirically by comparing measured deflections for over twenty full scale lateral load tests and the predicted deflections (Briaud 1997). For a pipe pile, the moment of inertia of the pile, $I\left(\mathrm{~m}^{4}, \mathrm{ft}^{4}\right)$, can be calculated by Eq. (3-15):

$$
I=\frac{\left(\pi D_{o}^{4}\right)}{64}-\frac{\left(\pi D_{i}^{4}\right)}{64}
$$

where, $D_{0}=$ outside diameter of the pile $(\mathrm{m}, \mathrm{ft})$

$D_{\mathrm{i}}=$ inside diameter of the pile $(\mathrm{m}, \mathrm{ft})$

The transfer length, $l_{0}$, is a parameter which comes from the differential equation. It has no physical meaning except that it indicates the relative stiffness between the pile and the soil in units of length. The transfer length $l_{0}$ can be computed by Eq. (3-16):

$$
l_{0}=\left(\frac{4 E I}{K_{s}}\right)^{1 / 4}
$$

where, $\mathrm{E}=$ modulus of elasticity for the pile material $\left(\mathrm{kPa}, \mathrm{lb} / \mathrm{ft}^{2}\right)$ 
If the embedded pile length, $L_{p}$, is larger than three times the transfer length, the pile can be treated as a long flexible pile. If $L_{p}<l_{0}$, the pile is short and rigid. Since most piles satisfy $L_{p} \geq 3 l_{0}$, the equations only for long flexible piles are considered in this report. The zero-shear depth, $D_{v}$, shown in Figure 3-3 can be determined by Eq. (3-17) depending on the value of $l_{0}$ for the pile:

$$
D_{v}=l_{0} \tan ^{-1}\left(\frac{1}{1+\frac{2 M_{0}}{l_{0} H_{0}}}\right), \text { if } L_{p} \geq 3 l_{0}
$$

where, $L_{p}=$ embedded pile length $(\mathrm{m}, \mathrm{ft})$

$H_{0}=$ applied horizontal load at the ground surface $(\mathrm{kN}, \mathrm{lbs})$

$M_{0}=$ applied moment at the ground surface $(\mathrm{kN}-\mathrm{m}, \mathrm{lbs}-\mathrm{ft})=H_{0} h$

$h=$ height of the point of application of the load, $H_{0}$ above ground surface $(\mathrm{m}, \mathrm{ft})$

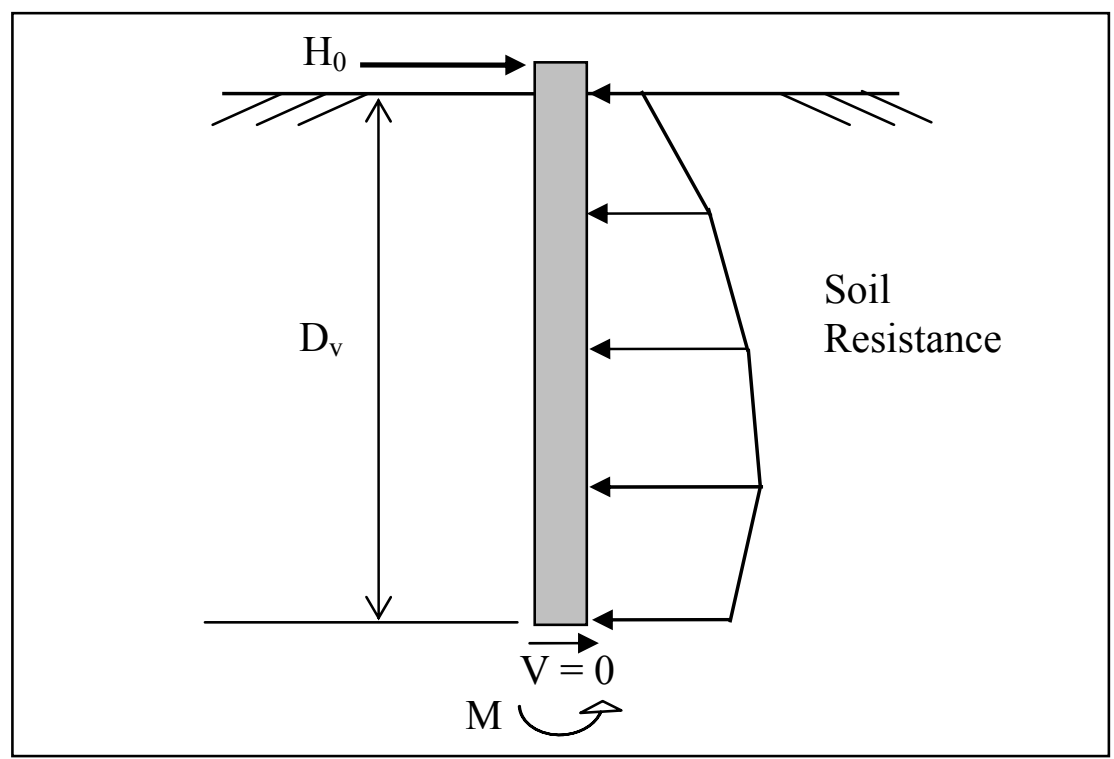

Figure 3-3. Free body diagram of pile down to zero-shear depth (Briaud 1997)

The ultimate lateral capacity of the pile with respect to soil capacity, $H_{o u}$, is computed by Eq. (3-18) (Briaud 1997):

$$
H_{o u}=0.75 P_{L} D_{o} D_{v}
$$


$P_{L}$ is the pre-boring pressuremeter (PMT) limit pressure within $D_{v}\left(\mathrm{kPa}, \mathrm{lb} / \mathrm{ft}^{2}\right)$. If $P_{L}$ is not available from PMT tests at the site, then the following correlations can be used with reduced accuracy:

$P_{L}(\mathrm{kPa})=47.9 N_{S P T}($ blow $/ 30 \mathrm{~cm})$, or $P_{L}(\mathrm{tsf})=0.5 N_{S P T}($ blow $/ \mathrm{ft})($ Briaud 1992)

In addition to the lateral capacity of the pile, both the deflections of the pile at the ground surface and the pile head should be checked and satisfy a certain limit. A deflection of 0.5 inches is a common limit of deflection for many structures. For that reason it is used in this report as a target value. The deflection of a long flexible pile at the ground surface can be calculated by Eq. (3-19) (KNR 1999) and should be less than 0.5 in.:

$$
y_{0}=\frac{\left(1+h / l_{0}\right) H_{0} l_{0}^{3}}{2 E I}
$$

where, $h=$ height of the pile above the ground surface $(\mathrm{m}, \mathrm{ft})$

The deflection at the long flexible pile head can be obtained by Eq. (3-20) (KNR 1999):

$$
y_{h}=\frac{\left\lfloor\left(1+h / l_{0}\right)^{3}+0.5\right\rfloor H_{0} l_{0}{ }^{3}}{3 E I}
$$

where, $h=$ height of the pile above the ground surface $(\mathrm{m}, \mathrm{ft})$

Finally, the maximum bending moment, $M_{\max }$ in the pile should be less than or equal to the allowable moment for the pile. The value of $M_{\max }$ for a long flexible pile can be calculated by Eq. (3-21) (KNR 1999):

$$
M_{\max }=\frac{H_{0} l_{0}}{2} \sqrt{\left(1+2 h / l_{0}\right)^{2}+1} e^{-\left(Z_{\max } / l_{0}\right)}
$$

where, $Z_{\max }=D_{v}$ since $M_{\max }$ occurs where the shear stress is equal to zero

The equation for $M_{\max }$ for a short and rigid pile is not included since all of the piles calculated in this report turned out to be long flexible piles. Although the maximum bending moments are computed, they are not checked against the yield moment of the pile material. In other words, the lateral pile capacity is checked only against failure of the surrounding soil, not failure of the pile itself. 
The procedures for the lateral pile capacity in the fine grained soils are almost the same as those in the case of coarse grained soils. The average pressuremeter modulus, $E_{0}$ and the pre-boring pressuremeter limit pressure within $D_{\mathrm{v}}, P_{L}$ in the fine grained soils can be determined by Eq. (3-22) and Eq. (3-23), respectively (Briaud 1992);

$$
\begin{aligned}
& E_{0}=100 s_{u} \\
& P_{L}=7.5 s_{u}
\end{aligned}
$$

Once these two values are obtained, the same procedures as described in the previous section should be applied to check the lateral pile capacity.

\subsubsection{Fixed Head Case}

The spring constant, $K_{\mathrm{s}}$, is the ratio of the lateral resistance of the soil per unit length of a pile to the lateral displacement of the pile. It can be obtained by Eq. (3-24) (Briaud 1997):

$$
K_{s}=2.3 E_{0}
$$

$E_{0}$ is the first load pressuremeter (PMT) modulus and can be obtained by using the following correlations if PMT tests are not available:

$$
\begin{aligned}
E_{0}(\mathrm{kPa}) & =383 N_{S P T}(\text { blow } / 30 \mathrm{~cm}), \text { or } E_{0}(\mathrm{tsf})=4 N_{S P T}(\text { blow } / \mathrm{ft})(\text { Briaud 1992) } \\
& =\text { average pressuremeter modulus }(\mathrm{kPa}, \mathrm{tsf})
\end{aligned}
$$

where, $N_{\mathrm{SPT}}=$ blow count in Standard Penetration Test

The moment of inertia of the pipe pile, $I\left(\mathrm{~m}^{4}, \mathrm{ft}^{4}\right)$, can be calculated by Eq. (3-25):

$$
I=\frac{\left(\pi D_{0}^{4}\right)}{64}-\frac{\left(\pi D_{i}^{4}\right)}{64}
$$

where, $D_{0}=$ outside diameter of the pile $(\mathrm{m}, \mathrm{ft})$

$$
D_{\mathrm{i}}=\text { inside diameter of the pile }(\mathrm{m}, \mathrm{ft})
$$

The transfer length, $l_{0}$, is a function of the relative stiffness between the pile and the soil, and it can be computed by Eq. (3-26):

$$
l_{0}=\left(\frac{4 E I}{K_{s}}\right)^{1 / 4}
$$


where, $E=$ modulus of elasticity for the pile material $\left(\mathrm{kPa}, \mathrm{lb} / \mathrm{ft}^{2}\right)$

The moment at the pile head can be computed by Eq. (3-27):

$$
M_{h}=-0.5\left(1+\frac{h}{l_{0}}\right) H_{0} l_{0}
$$

where, $H_{0}=$ applied horizontal load $(\mathrm{kN}, \mathrm{lbs})$

If the embedded pile length, $L_{p}$, is larger than three times of the transfer length, the pile can be treated as a long flexible pile. If $L_{p}<l_{0}$, the pile is short and rigid. Since most piles satisfy $L_{p} \geq 3 l_{0}$, the equations only for long flexible piles are considered in this report. The zero-shear depth, $D_{\mathrm{v}}$, can be determined by Eq. (3-28) (KNR 1999) depending on the value of $l_{0}$ for the pile:

$$
D_{v}=l_{0} \tan ^{-1}\left(\frac{l_{0}}{h}\right), \quad \text { if } L_{p} \geq 3 l_{0}
$$

where, $L_{p}=$ embedded pile length $(\mathrm{m}, \mathrm{ft})$

The lateral capacity of the pile, $H_{\mathrm{ou}}$, is computed by Eq. (3-29) (Briaud 1997):

$$
H_{o u}=0.75 P_{L} D_{o} D_{v}
$$

$P_{L}$ is the pre-boring pressuremeter (PMT) limit pressure within $D_{v}\left(\mathrm{kPa}, \mathrm{lb} / \mathrm{ft}^{2}\right)$. If $P_{L}$ is not available from PMT tests at the site, then the following correlations can be used with reduced accuraty:

$P_{L}(\mathrm{kPa})=47.9 N_{S P T}($ blow $/ 30 \mathrm{~cm})$, or $P_{L}(\mathrm{tsf})=0.5 N_{S P T}($ blow $/ \mathrm{ft})($ Briaud 1992)

As checked in the free head case, the deflections of the pile at the ground surface and the pile head should meet the 0.5 in. criterion. The deflection of a long flexible pile at the ground surface can be calculated by Eq. (3-30) (KNR 1999):

$$
y_{0}=\frac{\left(1+h / l_{0}\right) H_{0} l_{0}{ }^{3}}{4 E I}
$$

The deflection at the long flexible pile head can be obtained by Eq. (3-31):

$$
y_{h}=\frac{\left[\left(1+h / l_{0}\right)^{3}+2\right] H_{0} l_{0}{ }^{3}}{12 E I}
$$

where, $h=$ height of the pile above the ground surface $(\mathrm{m}, \mathrm{ft})$ 
Finally, the maximum bending moment, $M_{\max }$ in the pile should be less than or equal to the allowable moment for the pile. The value of $M_{\max }$ for a long flexible pile can be calculated by Eq. (3-32):

$$
M_{\max }=0.5 H_{0} l_{0} e^{-\left(Z_{\max } / l_{0}\right)} \sqrt{\left[1+\left(h / l_{0}\right)^{2}\right]}
$$

where, $Z_{\max }=D_{v}$ since $M_{\max }$ occurs where the shear stress is equal to zero

The equation of $M_{\max }$ for a short and rigid pile is not included since all of the piles calculated in this report turned to be long flexible. Although the maximum bending moments are computed, these are not checked with the yield moment of the pile material. In other words, the lateral pile capacities are checked only against failure of the surrounding soil.

The procedures for lateral pile capacity in fine grained soils are almost the same as those in coarse grained soils. In the absence of site specific pressuremeter data, the average pressuremeter modulus, $E_{0}$ and the pre-boring pressuremeter limit pressure, $P_{L}$ within $D_{\mathrm{v}}$, in fine grained soils can be determined by Eq. (3-33) and Eq. (3-34), respectively with reduced precision (Briaud 1992);

$$
\begin{aligned}
& E_{0}=100 s_{u} \\
& P_{L}=7.5 s_{u}
\end{aligned}
$$

Once these two values are obtained, the same procedures as described in the previous section should be applied to check the lateral pile capacity.

\subsubsection{Pile Capacity Check}

Once the axial and lateral pile capacities are estimated, they should be compared with the applied loads to check if the pile is safe against the loads. There are two different methods used extensively in the field: Load and Resistance Factor Design (LRFD) and Working Stress Design (WSD). 


\subsubsection{Load and Resistance Factor Design (LRFD) Method}

The Load and Resistance Factor Design (LRFD) method is based on a reliability approach to provide a more uniform level of safety on both loads and resistance. The LRFD factors are developed on the basis of a probability of failure varying between 0.0005 to 0.001 . In the LRFD method the applied loads are multiplied by load factors, $\lambda_{\mathrm{i}}$ which are equal or larger than 1 . The resistances are multiplied by resistance factors, $\phi_{\mathrm{i}}$ which are equal or less than 1 . The magnitude of these factors depends on the types of loads and the types of resistance components, respectively. The $\lambda_{\mathrm{i}}$ and $\phi_{\mathrm{i}}$ values are found in various guidelines including AASHTO and API RP2A. All calculations of driven pile capacities in this report followed API RP2A-LRFD (2003), and these values are shown in Table 2.3. The worst case among the three different conditions in Table 3-3 should be checked with correspondingly factored resistance. For bored piles, the values of load factors are obtained from those values for driven piles, and the values of resistance factors in Table 3-4 can be used.

Table 3-3. Load and resistance factors for driven piles (API RP2A-LRFD, 2003)

\begin{tabular}{|l|l|c|}
\hline \multicolumn{1}{|c|}{ Load Condition } & \multicolumn{1}{c|}{ Load Factors } & Resistance Factor \\
\hline Gravity Loads & $1.3 D L+1.5 L L$ & 0.70 \\
\hline Operating environmental & $1.3 D L+1.5 L L+1.2 W_{\mathrm{o}}$ & 0.70 \\
\hline Extreme environmental & $1.1 D L+1.1 L L+1.35 W_{\mathrm{e}}$ & 0.80 \\
\hline Lateral Capacity & - & 0.75 \\
\hline
\end{tabular}

Note: $D L=$ dead load; $L L=$ live load;

$W_{\mathrm{o}}=$ wind load for operating environmental condition;

$W_{\mathrm{e}}=$ wind load for extreme environmental condition

Table 3.4. Recommended resistance factors for bored piles (ADSC, 1999)

\begin{tabular}{|l|l|c|c|}
\hline \multirow{2}{*}{ Load Condition } & \multirow{2}{*}{ Capacity Term } & \multicolumn{2}{|c|}{ Resistance Factor } \\
\cline { 3 - 4 } & & Sand & Clay \\
\hline \multirow{3}{*}{ Operating environmental } & End Bearing & 0.50 & 0.55 \\
\cline { 2 - 4 } & Skin Friction & 0.65 & 0.65 \\
\cline { 2 - 4 } & Uplift & 0.65 & 0.55 \\
\hline Extreme environmental & Overall & 1.00 & 1.00 \\
\hline Lateral Capacity & Overall & \multicolumn{2}{|c|}{0.75} \\
\hline
\end{tabular}


According to API RP2A-LRFD (2003), “The operating environmental condition should be representative of moderately severe conditions at the platform. Typically, a 1year to 5-year winter storm is used as an operating wind condition in the Gulf of Mexico. On the other hand, the extreme environmental condition uses a 100 -year return period event. Return period means the average interval of time between exceedances of the magnitude of an event."

The general equation in the LRFD method can be expressed as:

$$
\sum \lambda_{i} \times L_{i}(\text { Loads })=\sum \phi_{i} \times R_{i}(\text { Resistance })
$$

where, $\lambda_{i}=$ load factors $(\geq 1.0)$

$$
\phi_{i}=\text { resistance factors }(\leq 1.0)
$$

For the pile capacity check, the appropriate factors for the resistance (capacity) obtained in the previous sections should be selected according to the guideline. Then, the factored resistance is to be compared with the factored loads and it should be larger or equal to the factored loads.

\subsubsection{Working Stress Design (WSD) Method}

Working Stress Design (WSD) is a traditional method to achieve a level of conservatism against various uncertainties in many aspects. In the WSD method, the factor of safety is employed to reduce the risk level against failure and it is the ratio of resistance to the applied load:

$$
\text { Factor of Safety }(S F)=\frac{\text { Resistance }(R)}{\operatorname{Load}(L)}
$$

The allowable pile capacities are determined by dividing the ultimate pile capacity by the proper factor of safety. The API RP2A-WSD recommends the following minimum values for driven piles in Table 3-5 depending on the load condition. For bored piles the values in Table 3-6 can be used according to the ADSC (1999). 
Table 3-5. Recommended factor of safety for driven piles (API RP2A-WSD, 2000)

\begin{tabular}{|l|c|}
\hline \multicolumn{1}{|c|}{ Load Condition } & Factor of Safety \\
\hline Operating environmental conditions & 2.0 \\
\hline Extreme environmental conditions & 1.5 \\
\hline Uplift (pullout) conditions & 2.0 \\
\hline Lateral Capacity & 3.0 \\
\hline
\end{tabular}

Table 3-6. Recommended factor of safety for bored piles (ADSC, 1999)

\begin{tabular}{|l|c|}
\hline \multicolumn{1}{|c|}{ Load Condition } & Factor of Safety \\
\hline Operating environmental conditions & 3.0 \\
\hline Extreme environmental conditions & 2.0 \\
\hline Uplift (pullout) conditions & 3.0 \\
\hline Lateral Capacity & 3.0 \\
\hline
\end{tabular}

Briaud (1997) recommend a factor of safety of 3 for their lateral capacity calculation method. In the case of LRFD, it is decided to use a resistance factor for lateral capacity equal to 0.75 . This is a relatively high resistance factor because the data shown by Briaud (1997) indicates little scatter in the predicted vs. measured comparison. For the pile capacity check, the actual resistance (capacity) obtained in the previous sections is to be divided by the actual loads. It becomes the factor of safety for the pile and it should be higher than the recommended value.

\subsection{Decision Analysis}

In general, it is almost impossible to predict with certainty what the best result of each strategy will be because there are many uncertainties in real problems. Therefore, formal analysis is required to consider many complex problems. The goal of decision analysis is to structure and simplify the task of making hard decisions through quantitative basis (Jimenez et al. 2003). This approach provides logical analysis of the alternatives and quantitative rationale for the recommendation. Decision analysis is 
usually concerned with multiple conflicting objectives for many real world problems and, therefore, it is simply not true that "qualitatively speaking, business decisions are simple because the objective function is crystal clear (Keeney and Raiffa 1976).”

According to Keeney and Raiffa (1976) and Keeney (1992), the simple paradigm of decision analysis can be summarized in a five-step process as follows:

1. Preanalysis: the problem has been identified and the viable alternatives are given.

2. Structural analysis: the decision-maker structures the problem which includes specifying objectives, attributes, and attributes scales.

3. Uncertainty analysis: the decision-maker assigns probabilities to the branches emanating from chance nodes. These assignments are based on past empirical data and expert judgment.

4. Utility or value analysis: the decision-maker quantifies his/her preferences and then converts these preferences into utility numbers. The assignment of utility numbers to consequences must be such that the maximization of expected utility becomes the appropriate criterion for the decision-maker's optimal action.

5. Optimization Analysis: once decision-maker assigns utilities, he/she calculates his/her optimal strategy - the strategy that maximizes expected utility. There are various techniques to obtain an optimal strategy for a specific problem.

\subsubsection{The Assumption of Utility Function}

In order to be able to decompose the general multi-attribute utility function with $\mathrm{i}$ attributes into a simple functional form of the $\mathrm{i}$ individual attributes, two assumptions about the nature of the decision-maker's preferences for the underlying attributes must be specified and verified (Hardaker 2004). These two assumptions are mutually preferential independence and utility independence. The preferential independence concerns only ordinal preferences and no probabilistic elements are involved (Keeney and Raiffa 1976). For example, suppose there are two attributes, X and Y. If preferences for levels of attribute $\mathrm{X}$ do not depend on the level of attribute $\mathrm{Y}$, an attribute $\mathrm{X}$ is said to be preference independent of another attribute Y. Utility independence, on the other 
hand, concerns the cardinal preferences of the decision-maker (Keeney and Raiffa 1976). For example, if preferences for uncertain choices such as lotteries involving different levels of attribute $\mathrm{X}$ do not depend on the level of attribute $\mathrm{Y}$, an attribute $\mathrm{X}$ is said to be utility independent of another attribute Y. Full mutual utility independence is almost impossible in reality, but the assumption is commonly made since to do otherwise would make the analysis too difficult (Hardaker 2004). It is very important to ascertain whether any of the preferential independence or utility independence assumptions discussed above is appropriate for this research.

\subsubsection{Forms of the Utility Function}

If mutual preferential and utility independence are satisfied, it is possible to define the multi-attribute utility function in the general form (Clemen and Reilly 2001):

$$
U\left(x_{1}, x_{2}, \ldots, x_{I}\right)=U\left\{u_{1}\left(x_{1}\right), u_{2}\left(x_{2}\right), \ldots, u_{I}\left(x_{I}\right)\right\}
$$

Once each single-attribute utility function $u_{i}\left(x_{i}\right)$ is derived for its attribute measure, these individual utility values are combined in some way into a final utility value.

If single-attribute utility functions $u_{i}\left(x_{i}\right)$ are scaled from zero to one, and if $U$ is also scaled from zero to one, the function $U$ is either of the additive form (Hardaker 2004):

$$
U\left(x_{1}, x_{2}, \ldots, x_{I}\right)=\sum_{i=1}^{I} k_{i} u_{i}\left(x_{i}\right)
$$

or of the multiplicative form (Hardaker 2004):

$$
U\left(x_{1}, x_{2}, \ldots, x_{I}\right)=\left\{\prod_{i=1}^{I}\left(K \cdot k_{i} u_{i}\left(x_{i}\right)+1\right)-1\right\} / K
$$

where $u_{i}\left(x_{i}\right)$ is a single-attribute utility function scaled from 0 to $1, k_{i}$ is a scaling factor between zero and one for $u_{i}\left(x_{i}\right) . K$ is another scaling constant and the value of $K$ depends on the values $k_{i}$. If $\Sigma k_{i}=1$, then $K=0$ and $U$ takes the additive form as expressed in Eq. (3-38) and it indicates there is no interaction between each attribute. In contrast, if $\Sigma k_{i} \neq$ 1, then $K \neq 0$ and $U$ takes the multiplicative form as expressed in Eq. (3-39). If $K$ is greater than 0 , then the attributes interact destructively so that a low utility for one 
attribute can result in a low overall utility $U$. On the other hand, when $K$ is less than 0 , the attributes interact constructively so that a high individual attribute utility results in a high overall utility $U$. Keeney (1974) describes more detail information about the derivation of $K$ from the $k_{i}$ values in the multiplicative case.

\subsubsection{Sensitivity Analysis}

Sensitivity analysis for multi-attribute utility problems can be categorized based on the number of times an optimization routine needs to be run to analyze sensitivity (Guikema and Milke 2003). If various individuals have distinct weight combinations for multi-attribute utility problems, each combination could be given as a discrete weight combination to the optimization routine and any result change in the technology selected would indicate sensitivity to an individual's choice of weight combination. In this case, not only does relatively few optimization need to be run, but also relatively little postprocessing of the optimization results is needed to evaluate sensitivity (Guikema and Milke 2003). The sensitivity analysis for discrete weight combinations of multi-attribute utility problems has been addressed many times in the literature. Call and Merkhofer (1988), for example, developed one approach to sensitivity analysis using predefined weight combinations (i.e., high and low for each attribute).

On the other hand, if decision-makers do not feel confident enough in their assessments to specify precise values, uncertainties of input parameters such as the weights of each attribute in multi-attribute utility problems can arise. In this case the proper values can lie anywhere within a possibly wide range of values specified by the decision-makers. For this type of sensitivity analysis, multiple optimizations need to be run and the breakpoints become important. In this research, for example, the breakpoints where the optimal drilling systems change are very important aspect. This type of sensitivity analysis is more difficult and time consuming than discrete sensitivity analysis. Significantly less has been addressed for this type of sensitivity analysis in the literature than for the discrete sensitivity analysis. 


\section{PARAMETRIC STUDY OF FOUNDATIONS FOR DRILL SITES}

\subsection{Foundation Options for Drill Sites}

After having several meetings with EFD foundation experts, some of possible foundation options for a drilling site containing the advantage and disadvantage associated with those options are identified as shown in Table 4-1.

Table 4-1. Foundation options for a drilling site

\begin{tabular}{|c|c|}
\hline 1. Gravel pad & Advantages \\
\hline \multirow{3}{*}{ 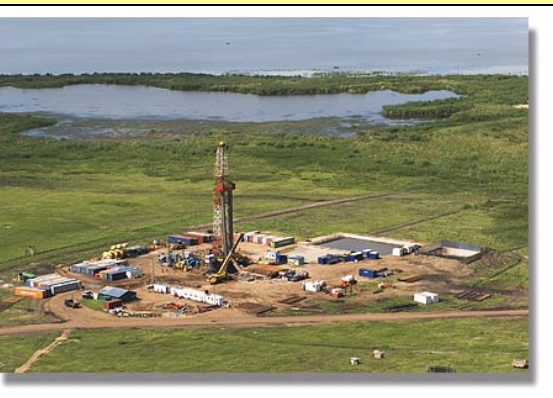 } & $\begin{array}{l}\text { - Easier and faster installation } \\
\text { - Maybe cheaper in construction stage }\end{array}$ \\
\hline & Disadvantages \\
\hline & $\begin{array}{l}\text { - Less environmentally friendly } \\
\text { - Non-resuable }\end{array}$ \\
\hline \multirow[t]{4}{*}{ 2. Composite mat } & Advantages \\
\hline & $\begin{array}{l}\text { - Easier and faster installation } \\
\text { - Great effect on small loading area } \\
\text { over soft soil }(\mathrm{E}<10 \mathrm{MPa})\end{array}$ \\
\hline & Disadvantages \\
\hline & $\begin{array}{l}\text { - Less effect on large loading area } \\
\text { over stiff soil }(\mathrm{E}>50 \mathrm{MPa})\end{array}$ \\
\hline 3. Spread footing & Advantages \\
\hline \multirow{3}{*}{ 焦 } & $\begin{array}{l}\text { - Simple and no discharge } \\
\text { - Uplift on marshes } \\
\text { - Easy to remove on rock }\end{array}$ \\
\hline & Disadvantages \\
\hline & $\begin{array}{l}\text { - No uplift on rock } \\
\text { - Suitable contact on rock } \\
\text { - Hard to remove on marsh }\end{array}$ \\
\hline
\end{tabular}


Table 4-1. Continued

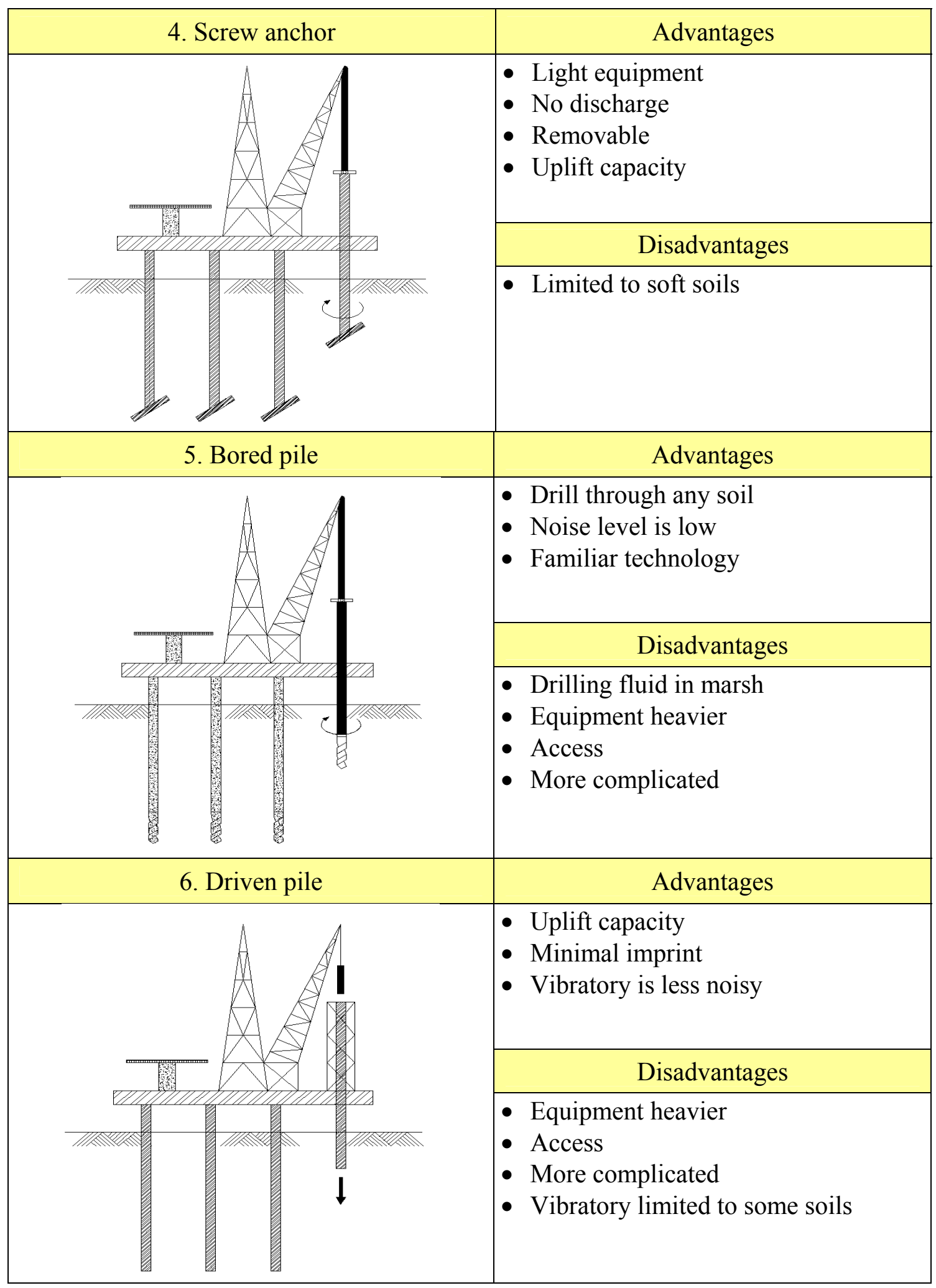


Among those foundation options, three different foundations (i.e., driven pile, bored pile, and composite mat) for drill sites are considered for the parametric study in the following Section 4.2 through 4.3. In order to encourage site location engineers to use environmentally friendly foundations such as elevated platforms and composite mat systems more often for their drilling sites instead of using gravel pads, the parametric study is an essential task in this research.

\subsection{Pile Foundation Designs for Low Impact Onshore Platforms}

Environmental issues are a significant part of every industry. The petroleum industry endeavors to minimize the existing environmental impact during drilling operations whether developing new resources or extending field in environmentally sensitive areas. For example, reducing the environmental footprint during drilling operations using a reusable Modular Platform and small mobile rig in the Arctic was demonstrated in 2003 by Anadarko and Noble's Subsidiary, Maurer technology Inc.. The objective was to drill in an ecologically sensitive area without disturbing the ground surface. The successful demonstration used a small mining rig to evaluate the potential of drilling for hydrates under the frozen tundra of the Alaska North Slope (Kadaster and Millheim 2004) and showed the usefulness of an onshore platform to drill in sensitive areas.

The objective of this study is to help the petroleum industry engineers to get a basic idea regarding pile designs of a platform for various platform weights and soil conditions in environmentally sensitive areas (e.g., desert environments and wetland applications). Use of a raised platform in environmentally sensitive areas will require the use of piles to support the elevated platform instead of gravel pads as used in a conventional platform. About one thousand different cases of pile capacity calculations are conducted depending on various soil types, pile types, and design methods. The results of these calculations are organized into a series of tables in order for the engineer to be able to easily choose an appropriate pile size for a given condition from these tables without performing an extensive pile design analysis. 


\subsubsection{Description of the General Case}

Anadarko's onshore platform in Alaska (Kadaster and Millheim 2004) is adopted for the foundation design of the general case. The platform consists of "bucket" modules (12.5 ft wide, $50 \mathrm{ft}$ long, and $3.5 \mathrm{ft}$ deep), piles for its leg, and drilling rig components. Figure 4-1 shows the dimension of one module, Figure 4-2 shows the plan view of several modules connected each other, and Figure 4-3 shows the cross sectional view of the platform. It is assumed that the mast is $90 \mathrm{ft} \mathrm{high,} 10 \mathrm{ft}$ long and the living quarter is $28 \mathrm{ft}$ high, $40 \mathrm{ft}$ long, respectively.

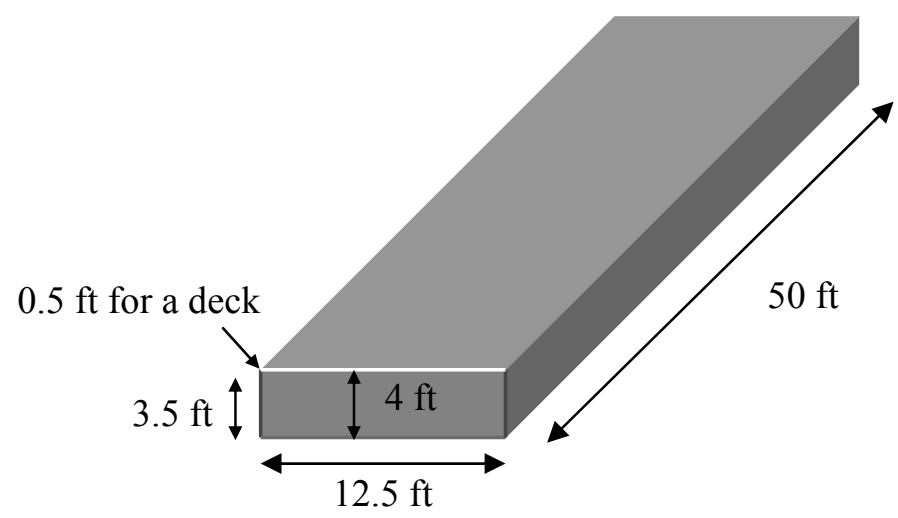

Figure 4-1. Module dimension

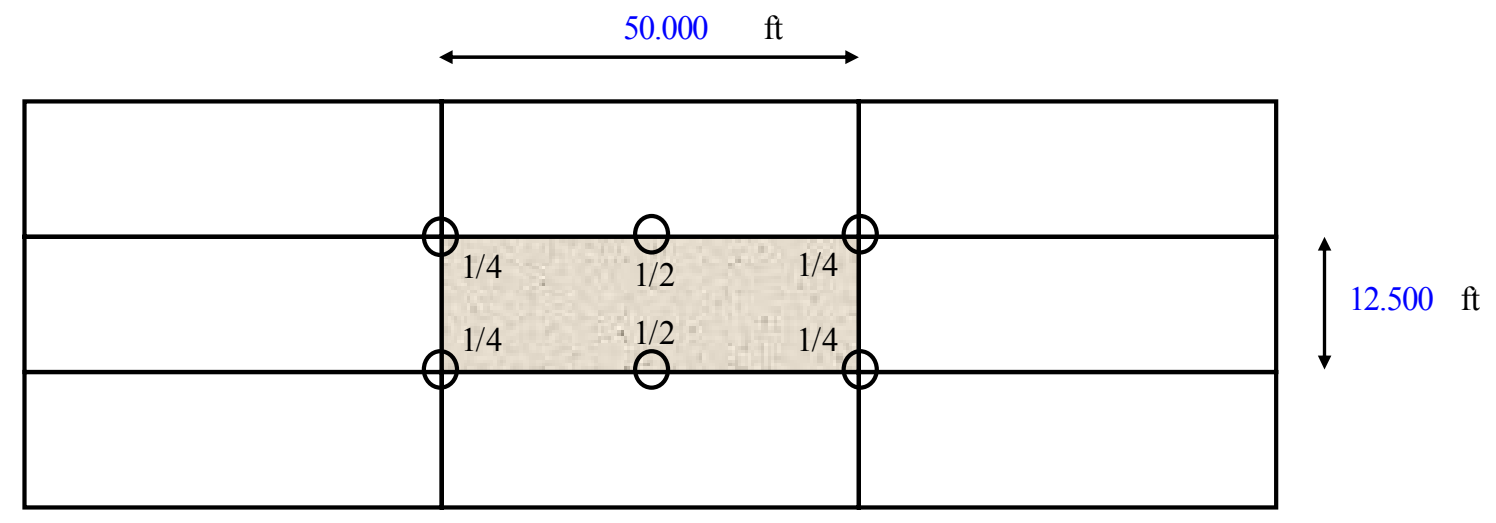

Figure 4-2. Plan view of modules 


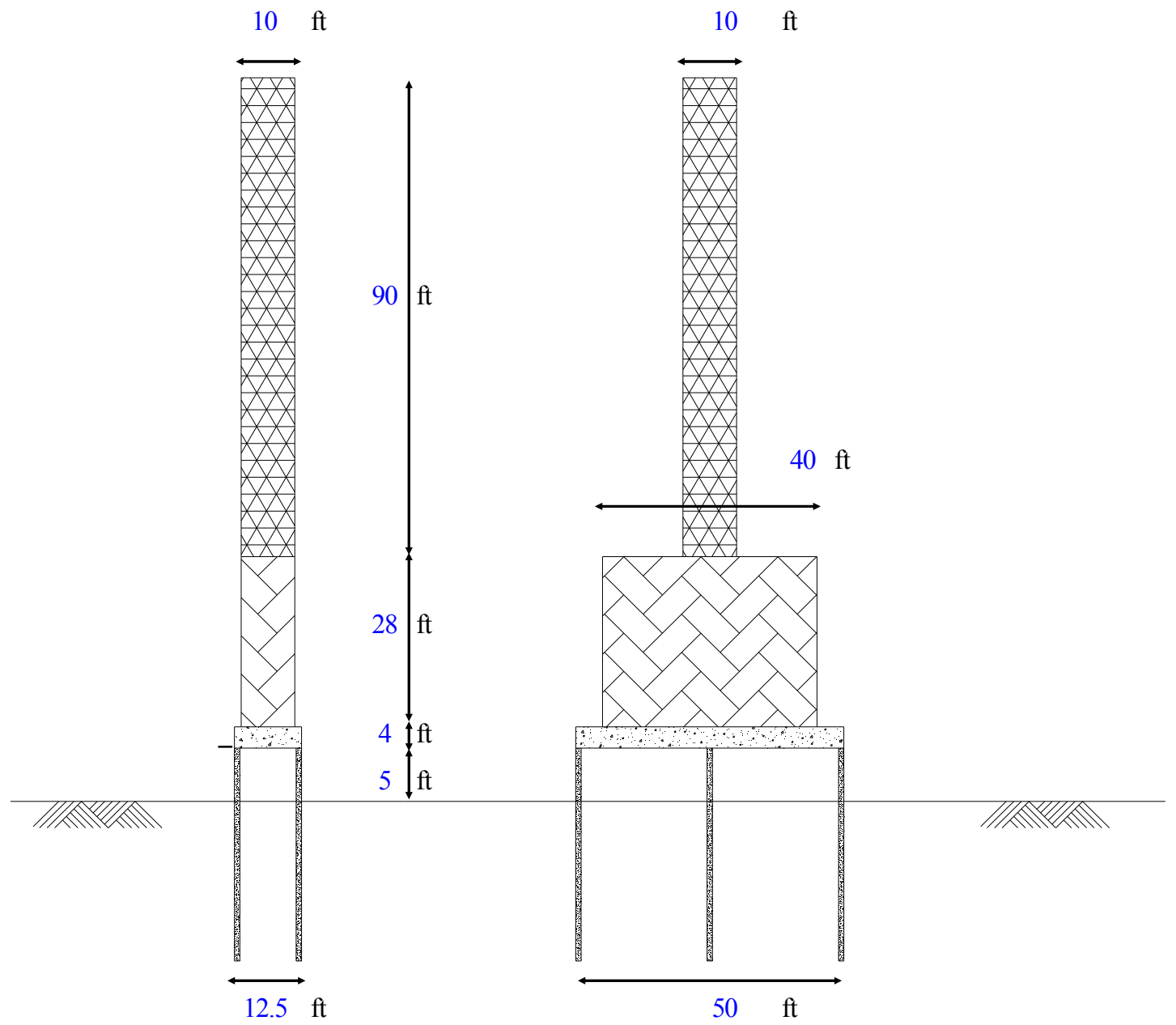

Figure 4-3 Cross section of the platform for one module in design

\subsubsection{Soil Conditions}

Pile capacities are strongly affected by the underlying soil type. If there is very dense sand under the ground, a pile will resist a much higher applied load than a pile in loose sand. Although it is highly desirable to calculate pile capacities in a site specific fashion, six typical types of soils are considered in this report. Furthermore, a homogeneous condition with respect to depth is assumed for simplicity in the calculations. The engineering properties of these soils are shown in Table 4-2. 
Table 4-2. Soil conditions of pile capacity calculations

\begin{tabular}{|c|c|c|c|c|c|}
\hline \multicolumn{3}{|c|}{ Gravels \& Sands } & \multicolumn{3}{c|}{ Silts \& Clays } \\
\hline $\begin{array}{c}\text { Type I } \\
\text { (very dense) }\end{array}$ & $\begin{array}{c}\text { Type II } \\
\text { (medium) }\end{array}$ & $\begin{array}{c}\text { Type III } \\
\text { (very loose) }\end{array}$ & $\begin{array}{c}\text { Type IV } \\
\text { (hard) }\end{array}$ & $\begin{array}{c}\text { Type V } \\
\text { (medium) }\end{array}$ & $\begin{array}{c}\text { Type VI } \\
\text { (soft) }\end{array}$ \\
\hline $\begin{array}{c}\gamma_{\text {sat }}=127 \mathrm{pcf} \\
\text { G.W.L }=20 \mathrm{ft}\end{array}$ & $\begin{array}{c}\gamma_{\mathrm{sat}}=120 \mathrm{pcf} \\
\text { G.W.L }=10 \mathrm{ft}\end{array}$ & $\begin{array}{c}\gamma_{\mathrm{sat}}=115 \mathrm{pcf} \\
\text { G.W.L }=0 \mathrm{ft}\end{array}$ & $\begin{array}{c}\gamma_{\mathrm{sat}}=127 \mathrm{pcf} \\
\text { G.W.L }=20 \mathrm{ft}\end{array}$ & $\begin{array}{c}\gamma_{\mathrm{sat}}=120 \mathrm{pcf} \\
\text { G.W.L }=10 \mathrm{ft}\end{array}$ & $\begin{array}{c}\gamma_{\mathrm{sat}}=115 \mathrm{pcf} \\
\mathrm{G} . \mathrm{W} . \mathrm{L}=0 \mathrm{ft}\end{array}$ \\
$\mathrm{N}_{\mathrm{SPT}}=50 \mathrm{bpf}$ & $\mathrm{N}_{\mathrm{SPT}}=30 \mathrm{bpf}$ & $\mathrm{N}_{\mathrm{SPT}}=10 \mathrm{bpf}$ & $s_{u}=2090 \mathrm{psf}$ & $s_{u}=1255 \mathrm{psf}$ & $s_{u}=0.25 \mathrm{P}_{0}$ \\
\hline
\end{tabular}

Note: $\mathrm{P}_{0}{ }^{\prime}=$ effective overburden pressure $(\mathrm{psf})$

G.W.L = ground water depth measured from the ground surface

\subsubsection{Weight Distribution on Platform}

For the general case, it is assumed that $65 \%$ of the total vertical loads are evenly distributed over 6 modules and that this load consists of dead load (30\%) and live load $(70 \%)$

The wind load is one of the primary sources of horizontal loads against a structure. According to API RP2A-LRFD (2003), wind load may be computed by Eq. $(4-1)$;

$$
W=\frac{\rho}{2} V^{2} C_{s} A
$$

where, $W=$ wind force, $V=$ wind speed

$C_{s}=$ dimensionless shape coefficient for perpendicular wind approach angles with respect to each projected area

$A=$ area of object perpendicular to the wind

$\rho=$ mass density of air at standard temperature and pressures

$$
=1.226 \mathrm{~kg} / \mathrm{m}^{3}=0.00238 \mathrm{lb} \cdot \mathrm{sec}^{2} / \mathrm{ft}^{4} \text { ) }
$$

The one hour mean wind speed at elevation $z$ can be calculated by Eq. (4-2);

$$
V(1 h r, z)=V\left(1 h r, z_{R}\right)\left(\frac{z}{z_{R}}\right)^{0.125}
$$

where, $V\left(1 h r, z_{R}\right)=$ one hour mean speed at the reference elevation $(\mathrm{m} / \mathrm{s}, \mathrm{ft} / \mathrm{s})$

$$
z_{R}=\text { reference elevation }(=10 \mathrm{~m} \text { or } 33 \mathrm{ft})
$$


According to API RP2A-LRFD (2003), the extreme wind speed to be considered in design for the Gulf of Mexico area is $49 \mathrm{~m} / \mathrm{s}$. In this report, $25 \mathrm{~m} / \mathrm{s}$ and $49 \mathrm{~m} / \mathrm{s}$ are assumed for operational and extreme wind speeds, respectively. More detailed load calculations in the general case can be found in APPENDIX A.

\subsubsection{Pile Capacity Check}

For the general case, the capacities of the driven steel pipe piles and bored piles are calculated in accordance with the LRFD and WSD methods. The step-by-step calculations can be found in APPENDIX A. First, the axial capacity is checked against the applied loads. Second, the lateral capacity is checked for the free head condition. Finally, the lateral capacity in the fixed head condition is evaluated.

\subsubsection{Results Summary}

Based on the pile capacity calculations in the general case, the following four tables (Table 4-3 4-6) provide a simple way to choose an appropriate pile size for a given condition. Once the soil type and the applied loads are known, the desirable pile size can be decided by following procedure;

1. Choose a design method: LRFD or WSD

2. Choose a pile type: driven or bored

3. Go to a table corresponding to the selected design method and pile type

4. Read the recommended diameter and length of the pile in the table 
Table 4-3. Recommended size of driven piles in the general case (LRFD)

\begin{tabular}{|c|c|c|c|c|c|c|c|}
\hline \multirow{3}{*}{$\begin{array}{l}\text { Weight of } \\
\text { Rigs \& } \\
\text { Accessories } \\
\text { (Unfactored) }\end{array}$} & & \multicolumn{3}{|c|}{ Sand \& Gravels } & \multicolumn{3}{|c|}{ Silts \& Clays } \\
\hline & & very loose & medium & very dense & soft & medium & hard \\
\hline & $\begin{array}{l}\text { Factored } \\
\text { max. vertica } \\
\text { loads on one }\end{array}$ & $\begin{array}{c}\gamma_{\mathrm{sat}}=115 \mathrm{pcf} \\
\text { G.W.L }=0 \mathrm{ft} \\
\mathrm{N}_{\mathrm{SPT}}=10 \mathrm{bpf}\end{array}$ & $\begin{array}{l}\gamma_{\mathrm{sat}}=120 \mathrm{pcf} \\
\text { G.W.L }=10 \mathrm{ft} \\
\mathrm{N}_{\mathrm{SPT}}=30 \mathrm{bpf}\end{array}$ & $\begin{array}{c}\gamma_{\mathrm{sat}}=127 \mathrm{pcf} \\
\text { G.W.L }=20 \mathrm{ft} \\
\mathrm{N}_{\mathrm{SPT}}=50 \mathrm{bpf}\end{array}$ & $\begin{array}{l}\gamma_{\mathrm{sat}}=115 \mathrm{pcf} \\
\text { G.W.L }=0 \mathrm{ft} \\
\mathrm{Su}=0.25 \mathrm{P}_{\mathrm{o}}{ }^{\prime}\end{array}$ & $\begin{array}{c}\gamma_{\mathrm{sat}}=120 \mathrm{pcf} \\
\text { G.W.L }=10 \mathrm{ft} \\
\mathrm{Su}=60 \mathrm{kPa}\end{array}$ & $\begin{array}{c}\gamma_{\text {sat }}=127 \mathrm{pcf} \\
\text { G.W.L }=20 \mathrm{ft} \\
\mathrm{Su}=100 \mathrm{kPa}\end{array}$ \\
\hline \multirow{2}{*}{1000 kips } & \multirow{2}{*}{178.0 kips } & $\mathrm{D}=24 \mathrm{in}$. & $\mathrm{D}=24 \mathrm{in}$. & $\mathrm{D}=12 \mathrm{in}$. & $\mathrm{D}=24 \mathrm{in}$ & $\mathrm{D}=24 \mathrm{in}$. & $\mathrm{D}=24 \mathrm{in}$. \\
\hline & & $\mathrm{L}=58 \mathrm{ft}$ & $\mathrm{L}=26 \mathrm{ft}$ & $\mathrm{L}=28 \mathrm{ft}$ & $\mathrm{L}=70 \mathrm{ft}$ & $\mathrm{L}=41 \mathrm{ft}$ & $\mathrm{L}=27 \mathrm{ft}$ \\
\hline \multirow{2}{*}{1500 kips } & \multirow{2}{*}{207.8 kips } & $\mathrm{D}=24 \mathrm{in}$. & $\mathrm{D}=24 \mathrm{in}$. & $\mathrm{D}=16 \mathrm{in}$. & $\mathrm{D}=24 \mathrm{in}$ & $\mathrm{D}=24 \mathrm{in}$. & $\mathrm{D}=24 \mathrm{in}$. \\
\hline & & $\mathrm{L}=64 \mathrm{ft}$ & $\mathrm{L}=30 \mathrm{ft}$ & $\mathrm{L}=21 \mathrm{ft}$ & $\mathrm{L}=75 \mathrm{ft}$ & $\mathrm{L}=47 \mathrm{ft}$ & $\mathrm{L}=32 \mathrm{ft}$ \\
\hline \multirow{2}{*}{2000 kips } & \multirow{2}{*}{237.6 kips } & $\mathrm{D}=24 \mathrm{in}$. & $\mathrm{D}=24 \mathrm{in}$. & $\mathrm{D}=20 \mathrm{in}$. & $\mathrm{D}=24 \mathrm{in}$ & $\mathrm{D}=24 \mathrm{in}$. & $\mathrm{D}=24 \mathrm{in}$. \\
\hline & & $\mathrm{L}=69 \mathrm{ft}$ & $\mathrm{L}=33 \mathrm{ft}$ & $\mathrm{L}=17 \mathrm{ft}$ & $\mathrm{L}=81 \mathrm{ft}$ & $\mathrm{L}=52 \mathrm{ft}$ & $\mathrm{L}=36 \mathrm{ft}$ \\
\hline \multirow{2}{*}{3000 kips } & \multirow{2}{*}{297.1 kips } & $\mathrm{D}=24 \mathrm{in}$ & $\mathrm{D}=24 \mathrm{in}$ & $\mathrm{D}=20 \mathrm{in}$. & $\mathrm{D}=24 \mathrm{in}$ & $\mathrm{D}=24 \mathrm{in}$. & $\mathrm{D}=24 \mathrm{in}$. \\
\hline & & $\mathrm{L}=79 \mathrm{ft}$ & $\mathrm{L}=40 \mathrm{ft}$ & $\mathrm{L}=21 \mathrm{ft}$ & $\mathrm{L}=91 \mathrm{ft}$ & $\mathrm{L}=63 \mathrm{ft}$ & $\mathrm{L}=45 \mathrm{ft}$ \\
\hline \multirow{2}{*}{4000 kips } & \multirow{2}{*}{356.7 kips } & $\mathrm{D}=24 \mathrm{in}$. & $\mathrm{D}=24 \mathrm{in}$ & $\mathrm{D}=20 \mathrm{in}$. & $\mathrm{D}=24 \mathrm{in}$ & $\mathrm{D}=24 \mathrm{in}$. & $\mathrm{D}=20 \mathrm{in}$. \\
\hline & & $\mathrm{L}=94 \mathrm{ft}$ & $\mathrm{L}=48 \mathrm{ft}$ & $\mathrm{L}=27 \mathrm{ft}$ & $\mathrm{L}=104 \mathrm{ft}$ & $\mathrm{L}=77 \mathrm{ft}$ & $\mathrm{L}=66 \mathrm{ft}$ \\
\hline
\end{tabular}

Table 4-4. Recommended size of driven piles in the general case (WSD)

\begin{tabular}{|c|c|c|c|c|c|c|c|}
\hline \multirow{3}{*}{$\begin{array}{l}\text { Weight of } \\
\text { Rigs \& } \\
\text { Accessories } \\
\text { (Unfactored) }\end{array}$} & & \multicolumn{3}{|c|}{ Sand \& Gravels } & \multicolumn{3}{|c|}{ Silts \& Clays } \\
\hline & & very loose & medium & very dense & soft & medium & hard \\
\hline & $\begin{array}{l}\text { Unfactored } \\
\text { max. vertical } \\
\text { loads on one }\end{array}$ & $\begin{array}{c}\gamma_{\mathrm{sat}}=115 \mathrm{pcf} \\
\text { G.W.L }=0 \mathrm{ft} \\
\mathrm{N}_{\mathrm{SPT}}=10 \mathrm{bpf}\end{array}$ & $\begin{array}{c}\gamma_{\mathrm{sat}}=120 \mathrm{pcf} \\
\text { G.W.L }=10 \mathrm{ft} \\
\mathrm{N}_{\mathrm{SPT}}=30 \mathrm{bpf}\end{array}$ & $\begin{array}{c}\gamma_{\mathrm{sat}}=127 \mathrm{pcf} \\
\text { G.W.L }=20 \mathrm{ft} \\
\mathrm{N}_{\mathrm{SPT}}=50 \mathrm{bpf}\end{array}$ & $\begin{array}{l}\gamma_{\mathrm{sat}}=115 \mathrm{pcf} \\
\mathrm{G} . \mathrm{W} \cdot \mathrm{L}=0 \mathrm{ft} \\
\mathrm{Su}=0.25 \mathrm{P}_{\mathrm{o}}{ }^{\prime}\end{array}$ & $\begin{array}{c}\gamma_{\mathrm{sat}}=120 \mathrm{pcf} \\
\text { G.W.L }=10 \mathrm{ft} \\
\mathrm{Su}=60 \mathrm{kPa}\end{array}$ & $\begin{array}{c}\gamma_{\mathrm{sat}}=127 \mathrm{pcf} \\
\text { G.W.L }=20 \mathrm{ft} \\
\mathrm{Su}=100 \mathrm{kPa}\end{array}$ \\
\hline \multirow{2}{*}{1000 kips } & \multirow{2}{*}{141.9 kips } & $\mathrm{D}=24 \mathrm{in}$. & $\mathrm{D}=24 \mathrm{in}$. & $\mathrm{D}=16 \mathrm{in}$. & $\mathrm{D}=24 \mathrm{in}$ & $\mathrm{D}=24 \mathrm{in}$. & $\mathrm{D}=24 \mathrm{in}$. \\
\hline & & $\mathrm{L}=57 \mathrm{ft}$ & $\mathrm{L}=25 \mathrm{ft}$ & $\mathrm{L}=20 \mathrm{ft}$ & $\mathrm{L}=68 \mathrm{ft}$ & $\mathrm{L}=39 \mathrm{ft}$ & $\mathrm{L}=26 \mathrm{ft}$ \\
\hline \multirow{2}{*}{1500 kips } & \multirow{2}{*}{169.0 kips } & $\mathrm{D}=24 \mathrm{in}$. & $\mathrm{D}=24 \mathrm{in}$. & $\mathrm{D}=16 \mathrm{in}$. & $\mathrm{D}=24 \mathrm{in}$ & $\mathrm{D}=24 \mathrm{in}$. & $\mathrm{D}=24 \mathrm{in}$. \\
\hline & & $\mathrm{L}=63 \mathrm{ft}$ & $\mathrm{L}=29 \mathrm{ft}$ & $\mathrm{L}=21 \mathrm{ft}$ & $\mathrm{L}=74 \mathrm{ft}$ & $\mathrm{L}=46 \mathrm{ft}$ & $\mathrm{L}=31 \mathrm{ft}$ \\
\hline \multirow{2}{*}{2000 kips } & \multirow{2}{*}{196.0 kips } & $\mathrm{D}=20 \mathrm{in}$. & $\mathrm{D}=24 \mathrm{in}$. & $\mathrm{D}=20 \mathrm{in}$. & $\mathrm{D}=20 \mathrm{in}$ & $\mathrm{D}=24 \mathrm{in}$. & $\mathrm{D}=24 \mathrm{in}$. \\
\hline & & $\mathrm{L}=79 \mathrm{ft}$ & $\mathrm{L}=33 \mathrm{ft}$ & $\mathrm{L}=17 \mathrm{ft}$ & $\mathrm{L}=89 \mathrm{ft}$ & $\mathrm{L}=52 \mathrm{ft}$ & $\mathrm{L}=36 \mathrm{ft}$ \\
\hline \multirow{2}{*}{3000 kips } & \multirow{2}{*}{250.2 kips } & $\mathrm{D}=24 \mathrm{in}$. & $\mathrm{D}=24 \mathrm{in}$. & $\mathrm{D}=20 \mathrm{in}$. & $\mathrm{D}=20 \mathrm{in}$ & $\mathrm{D}=20 \mathrm{in}$. & $\mathrm{D}=24 \mathrm{in}$. \\
\hline & & $\mathrm{L}=79 \mathrm{ft}$ & $\mathrm{L}=40 \mathrm{ft}$ & $\mathrm{L}=21 \mathrm{ft}$ & $\mathrm{L}=101 \mathrm{ft}$ & $\mathrm{L}=74 \mathrm{ft}$ & $\mathrm{L}=45 \mathrm{ft}$ \\
\hline \multirow{2}{*}{4000 kips } & \multirow{2}{*}{304.4 kips } & $\mathrm{D}=24 \mathrm{in}$. & $\mathrm{D}=20 \mathrm{in}$. & $\mathrm{D}=16$ in. & $\mathrm{D}=24 \mathrm{in}$ & $\mathrm{D}=24 \mathrm{in}$. & $\mathrm{D}=24 \mathrm{in}$. \\
\hline & & $\mathrm{L}=93 \mathrm{ft}$ & $\mathrm{L}=58 \mathrm{ft}$ & $\mathrm{L}=37 \mathrm{ft}$ & $\mathrm{L}=104 \mathrm{ft}$ & $\mathrm{L}=77 \mathrm{ft}$ & $\mathrm{L}=56 \mathrm{ft}$ \\
\hline
\end{tabular}


Table 4-5. Recommended size of bored piles in the general case (LRFD)

\begin{tabular}{|c|c|c|c|c|c|c|c|}
\hline \multirow{3}{*}{$\begin{array}{l}\text { Weight of } \\
\text { Rigs \& } \\
\text { Accessories } \\
\text { (Unfactored) }\end{array}$} & \multirow{3}{*}{\begin{tabular}{|l} 
Soil types \\
Factored \\
max. vertical \\
loads on one pile
\end{tabular}} & \multicolumn{3}{|c|}{ Sand \& Gravels } & \multicolumn{3}{|c|}{ Silts \& Clays } \\
\hline & & very loose & medium & very dense & soft & medium & hard \\
\hline & & $\begin{array}{c}\gamma_{\mathrm{sat}}=115 \mathrm{pcf} \\
\text { G.W.L }=0 \mathrm{ft} \\
\text { NSPT }=10 \mathrm{bpf}\end{array}$ & $\begin{array}{l}\gamma_{\mathrm{sat}}=120 \mathrm{pcf} \\
\text { G.W.L }=10 \mathrm{ft} \\
\text { NsPT }=30 \mathrm{bpf}\end{array}$ & $\begin{array}{l}\gamma_{\mathrm{sat}}=127 \mathrm{pcf} \\
\text { G.W.L }=20 \mathrm{ft} \\
\text { NsPT }=50 \mathrm{bpf}\end{array}$ & $\begin{array}{l}\gamma_{\mathrm{sat}}=115 \mathrm{pcf} \\
\mathrm{G} . \mathrm{W} . \mathrm{L}=0 \mathrm{ft} \\
\mathrm{Su}=0.25 \mathrm{P}_{\mathrm{o}}^{\prime}\end{array}$ & $\begin{array}{c}\gamma_{\mathrm{sat}}=120 \mathrm{pcf} \\
\text { G.W.L }=10 \mathrm{ft} \\
\mathrm{Su}=60 \mathrm{kPa}\end{array}$ & $\begin{array}{c}\gamma_{\mathrm{sat}}=127 \mathrm{pcf} \\
\text { G.W.L }=20 \mathrm{ft} \\
\mathrm{Su}=100 \mathrm{kPa}\end{array}$ \\
\hline \multirow{2}{*}{1000 kips } & \multirow{2}{*}{$178.0 \mathrm{kips}$} & $\mathrm{D}=24 \mathrm{in}$. & $\mathrm{D}=20 \mathrm{in}$. & $\mathrm{D}=16$ in. & $\mathrm{D}=24 \mathrm{in}$. & $\mathrm{D}=24$ in. & $\mathrm{D}=24 \mathrm{in}$. \\
\hline & & $\mathrm{L}=38 \mathrm{ft}$ & $\mathrm{L}=19 \mathrm{ft}$ & $\mathrm{L}=19 \mathrm{ft}$ & $\mathrm{L}=85 \mathrm{ft}$ & $\mathrm{L}=41 \mathrm{ft}$ & $\mathrm{L}=24 \mathrm{ft}$ \\
\hline \multirow{2}{*}{1500 kips } & \multirow{2}{*}{207.8 kips } & $\mathrm{D}=24 \mathrm{in}$. & $\mathrm{D}=20 \mathrm{in}$. & $\mathrm{D}=20$ in. & $\mathrm{D}=24 \mathrm{in}$. & $\mathrm{D}=24$ in. & $\mathrm{D}=24 \mathrm{in}$. \\
\hline & & $\mathrm{L}=46 \mathrm{ft}$ & $\mathrm{L}=26 \mathrm{ft}$ & $\mathrm{L}=19 \mathrm{ft}$ & $\mathrm{L}=97 \mathrm{ft}$ & $\mathrm{L}=53 \mathrm{ft}$ & $\mathrm{L}=31 \mathrm{ft}$ \\
\hline \multirow{2}{*}{2000 kips } & \multirow{2}{*}{237.6 kips } & $\mathrm{D}=24 \mathrm{in}$. & $\mathrm{D}=20 \mathrm{in}$. & $\mathrm{D}=16$ in. & $\mathrm{D}=24 \mathrm{in}$. & $\mathrm{D}=24 \mathrm{in}$. & $\mathrm{D}=20 \mathrm{in}$. \\
\hline & & $\mathrm{L}=54 \mathrm{ft}$ & $\mathrm{L}=32 \mathrm{ft}$ & $\mathrm{L}=31 \mathrm{ft}$ & $\mathrm{L}=109 \mathrm{ft}$ & $\mathrm{L}=66 \mathrm{ft}$ & $\mathrm{L}=48 \mathrm{ft}$ \\
\hline \multirow{2}{*}{3000 kips } & \multirow{2}{*}{$297.1 \mathrm{kips}$} & $\mathrm{D}=24 \mathrm{in}$ & $\mathrm{D}=24 \mathrm{in}$ & $\mathrm{D}=20$ in. & $\mathrm{D}=24 \mathrm{in}$ & $\mathrm{D}=24 \mathrm{in}$. & $\mathrm{D}=24 \mathrm{in}$ \\
\hline & & $\mathrm{L}=70 \mathrm{ft}$ & $\mathrm{L}=36 \mathrm{ft}$ & $\mathrm{L}=33 \mathrm{ft}$ & $\mathrm{L}=131 \mathrm{ft}$ & $\mathrm{L}=94 \mathrm{ft}$ & $\mathrm{L}=56 \mathrm{ft}$ \\
\hline \multirow{2}{*}{4000 kips } & \multirow{2}{*}{356.7 kips } & $\mathrm{D}=24 \mathrm{in}$. & $\mathrm{D}=24 \mathrm{in}$. & $\mathrm{D}=24 \mathrm{in}$. & $\mathrm{D}=24 \mathrm{in}$. & $\mathrm{D}=24$ in. & $\mathrm{D}=24 \mathrm{in}$. \\
\hline & & $\mathrm{L}=86 \mathrm{ft}$ & $\mathrm{L}=46 \mathrm{ft}$ & $\mathrm{L}=34 \mathrm{ft}$ & $\mathrm{L}=149 \mathrm{ft}$ & $\mathrm{L}=122 \mathrm{ft}$ & $\mathrm{L}=73 \mathrm{ft}$ \\
\hline
\end{tabular}

(where, $\mathrm{P}_{\mathrm{o}}{ }^{\prime}=$ effective overburden pressure, G.W.L. = ground water depth measured from the ground surface,

Table 4-6. Recommended size of bored piles in the general case (WSD)

\begin{tabular}{|c|c|c|c|c|c|c|c|}
\hline \multirow{3}{*}{$\begin{array}{l}\text { Weight of } \\
\text { Rigs \& } \\
\text { Accessories } \\
\text { (Unfactored) }\end{array}$} & & \multicolumn{3}{|c|}{ Sand \& Gravels } & \multicolumn{3}{|c|}{ Silts \& Clays } \\
\hline & & very loose & medium & very dense & soft & medium & hard \\
\hline & $\begin{array}{l}\text { Unfactored } \\
\text { max. vertical } \\
\text { loads on one }\end{array}$ & $\begin{array}{c}\gamma_{\mathrm{sat}}=115 \mathrm{pcf} \\
\text { G.W.L }=0 \mathrm{ft} \\
\mathrm{N}_{\mathrm{SPT}}=10 \mathrm{bpf}\end{array}$ & $\begin{array}{l}\gamma_{\mathrm{sat}}=120 \mathrm{pcf} \\
\text { G.W.L }=10 \mathrm{ft} \\
\mathrm{N}_{\mathrm{SPT}}=30 \mathrm{bpf}\end{array}$ & $\begin{array}{c}\gamma_{\mathrm{sat}}=127 \mathrm{pcf} \\
\text { G.W.L }=20 \mathrm{ft} \\
\mathrm{N}_{\mathrm{SPT}}=50 \mathrm{bpf}\end{array}$ & $\begin{array}{l}\gamma_{\mathrm{sat}}=115 \mathrm{pcf} \\
\mathrm{G} . \mathrm{W} . \mathrm{L}=0 \mathrm{ft} \\
\mathrm{Su}=0.25 \mathrm{P}_{\mathrm{o}}^{\prime}\end{array}$ & $\begin{array}{c}\gamma_{\text {sat }}=120 \mathrm{pcf} \\
\text { G.W.L }=10 \mathrm{ft} \\
\mathrm{Su}=60 \mathrm{kPa}\end{array}$ & $\begin{array}{c}\gamma_{\text {sat }}=127 \mathrm{pcf} \\
\text { G.W.L }=20 \mathrm{ft} \\
\mathrm{Su}=100 \mathrm{kPa}\end{array}$ \\
\hline \multirow{2}{*}{1000 kips } & \multirow{2}{*}{141.9 kips } & $\mathrm{D}=24 \mathrm{in}$. & $\mathrm{D}=24 \mathrm{in}$. & $\mathrm{D}=20 \mathrm{in}$. & $\mathrm{D}=24 \mathrm{in}$ & $\mathrm{D}=24 \mathrm{in}$. & $\mathrm{D}=24 \mathrm{in}$. \\
\hline & & $\mathrm{L}=53 \mathrm{ft}$ & $\mathrm{L}=24 \mathrm{ft}$ & $\mathrm{L}=21 \mathrm{ft}$ & $\mathrm{L}=108 \mathrm{ft}$ & $\mathrm{L}=65 \mathrm{ft}$ & $\mathrm{L}=39 \mathrm{ft}$ \\
\hline \multirow{2}{*}{1500 kips } & \multirow{2}{*}{169.0 kips } & $\mathrm{D}=24 \mathrm{in}$. & $\mathrm{D}=24 \mathrm{in}$. & $\mathrm{D}=20 \mathrm{in}$. & $\mathrm{D}=24 \mathrm{in}$ & $\mathrm{D}=24 \mathrm{in}$. & $\mathrm{D}=24 \mathrm{in}$. \\
\hline & & $\mathrm{L}=60 \mathrm{ft}$ & $\mathrm{L}=29 \mathrm{ft}$ & $\mathrm{L}=26 \mathrm{ft}$ & $\mathrm{L}=118 \mathrm{ft}$ & $\mathrm{L}=78 \mathrm{ft}$ & $\mathrm{L}=46 \mathrm{ft}$ \\
\hline \multirow{2}{*}{2000 kips } & \multirow{2}{*}{196.0 kips } & $\mathrm{D}=24 \mathrm{in}$. & $\mathrm{D}=24 \mathrm{in}$. & $\mathrm{D}=24 \mathrm{in}$. & $\mathrm{D}=24 \mathrm{in}$ & $\mathrm{D}=24 \mathrm{in}$. & $\mathrm{D}=24 \mathrm{in}$. \\
\hline & & $\mathrm{L}=68 \mathrm{ft}$ & $\mathrm{L}=34 \mathrm{ft}$ & $\mathrm{L}=23 \mathrm{ft}$ & $\mathrm{L}=128 \mathrm{ft}$ & $\mathrm{L}=91 \mathrm{ft}$ & $\mathrm{L}=54 \mathrm{ft}$ \\
\hline \multirow{2}{*}{3000 kips } & \multirow{2}{*}{250.2 kips } & $\mathrm{D}=24 \mathrm{in}$. & $\mathrm{D}=20 \mathrm{in}$. & $\mathrm{D}=20 \mathrm{in}$. & $\mathrm{D}=24 \mathrm{in}$ & $\mathrm{D}=24 \mathrm{in}$. & $\mathrm{D}=24 \mathrm{in}$. \\
\hline & & $\mathrm{L}=89 \mathrm{ft}$ & $\mathrm{L}=57 \mathrm{ft}$ & $\mathrm{L}=43 \mathrm{ft}$ & $\mathrm{L}=153 \mathrm{ft}$ & $\mathrm{L}=129 \mathrm{ft}$ & $\mathrm{L}=77 \mathrm{ft}$ \\
\hline \multirow{2}{*}{4000 kips } & \multirow{2}{*}{304.4 kips } & $\mathrm{D}=24 \mathrm{in}$. & $\mathrm{D}=24 \mathrm{in}$ & $\mathrm{D}=24 \mathrm{in}$. & $\mathrm{D}=24 \mathrm{in}$ & $\mathrm{D}=24 \mathrm{in}$. & $\mathrm{D}=24 \mathrm{in}$. \\
\hline & & $\mathrm{L}=114 \mathrm{ft}$ & $\mathrm{L}=59 \mathrm{ft}$ & $\mathrm{L}=44 \mathrm{ft}$ & $\mathrm{L}=174 \mathrm{ft}$ & $\mathrm{L}=166 \mathrm{ft}$ & $\mathrm{L}=99 \mathrm{ft}$ \\
\hline
\end{tabular}




\subsubsection{Description of an Elevated Platform with Rapid Rig}

In May 2006, National Oilwell Varco (NOV) began offering a smaller, fully automatic land drilling rig called "Rapid Rig." The total vertical load of Rapid Rig is used for the foundation calculation of the proposed modular platform. The load breakdown and the layout of Rapid Rig are shown in Table 4-7, Figure 4-4, and Figure 4-5, respectively. In this case, the operating environmental condition governs the foundation calculation.

Table 4-7. Load breakdown structure of Rapid Rig in operating condition

\begin{tabular}{|c|c|c|c|c|c|c|c|}
\hline \multirow{3}{*}{ No. } & \multirow{3}{*}{ COMPONENTS } & \multirow{3}{*}{$\begin{array}{c}\text { WEIGHTS [lbs] } \\
\text { (DEAD) }\end{array}$} & \multirow{3}{*}{$\begin{array}{c}\text { WEIGHTS [lbs] } \\
\text { (LIVE) }\end{array}$} & \begin{tabular}{c|} 
Factored \\
Weights [lbs]
\end{tabular} & \multicolumn{2}{|c|}{ Dimension } & \multirow{3}{*}{ Notes } \\
\hline & & & & $\mathrm{D} . \mathrm{L}=1.3$ & \multirow{2}{*}{ W } & \multirow{2}{*}{$\mathrm{L}$} & \\
\hline & & & & L.L $=1.5$ & & & \\
\hline 1. & Substructure/Drillfloor package & 80,000 & & 104,000 & 10 & 58.5 & \\
\hline 2. & Mast including installed equipment & 100,000 & & 130,000 & 18 & 25 & \\
\hline 3. & Drawworks package includes Accumulator unit & 70,000 & & 91,000 & 10 & 29 & \\
\hline 4. & Utilities Skid & 25,000 & & 32,500 & 10 & 28 & \\
\hline 5. & Service Skid & 20,000 & & 26,000 & 10 & 38.75 & \\
\hline 6. & Electrical Control House & 30,000 & & 39,000 & 10 & 42 & \\
\hline 7. & Generator House \#1 & 40,000 & & 52,000 & 10 & 27.5 & \\
\hline 8. & Generator House \#2 & 40,000 & & 52,000 & 10 & 27.5 & \\
\hline 9. & Air Compressor House & 30,000 & & 39,000 & 10 & 27.5 & \\
\hline 10. & Mud Pump \#1 & 55,000 & & 71,500 & 8.75 & 22 & \\
\hline 11. & Mud Pump \#2 & 55,000 & & 71,500 & 8.75 & 22 & \\
\hline 12. & Pipe Handling equipment & 35,000 & & 45,500 & 3 & 80 & \\
\hline \multirow{2}{*}{13.} & Control House skid including choke manifold & 23,000 & & 29,900 & 18 & 12.5 & \\
\hline & Choke Manifold hauled on same trailer & 15,000 & & 19,500 & 7.5 & 14 & \\
\hline \multirow{2}{*}{14.} & Mud Tank Skid \#1 (Empty) & 40,000 & & 52,000 & 11.25 & 55 & \\
\hline & Mud Tank Skid \#1 (Full) & & 204,750 & 307,125 & 11.25 & 55 & 375 barrels, 13 lbs/gal \\
\hline \multirow{2}{*}{15.} & Mud Tank Skid \#2 (Empty) & 40,000 & & 52,000 & 11.25 & 55 & \\
\hline & Mud Tank Skid \#2 (Full) & & 204,750 & 307,125 & 11.25 & 55 & 375 barrels, $13 \mathrm{lbs} / \mathrm{gal}$ \\
\hline \multirow{2}{*}{16.} & Water Tank (Empty) & 20,000 & & 26,000 & 7.5 & 45 & \\
\hline & Water Tank (Full) & & 139,440 & 209,160 & 7.5 & 45 & 400 barrels, $8.3 \mathrm{lbs} / \mathrm{ga}$ \\
\hline 17. & Work shop/Storage Skid & 20,000 & & 26,000 & 10 & 27.5 & \\
\hline 18. & Fuel Tank Skid & 10,000 & & 13,000 & 8 & 30 & \\
\hline 19. & Casing & & 530,000 & 795,000 & 18 & 25 & $53 \mathrm{lbs} / \mathrm{ft}, 10000 \mathrm{ft}$ \\
\hline 20. & Pipes & & 234,000 & 351,000 & 18 & 25 & $19.5 \mathrm{lbs} / \mathrm{ft}, 12000 \mathrm{ft}$ \\
\hline 21. & Collars & & 2,720 & 4,080 & 18 & 25 & $80 \mathrm{lbs} / \mathrm{ft}, 34 \mathrm{ft}$ \\
\hline 22. & Drill collars & & 60,000 & 90,000 & 18 & 25 & $6000 \mathrm{lbs} \times 10$ \\
\hline \multicolumn{2}{|c|}{ Total Weights without Casing } & $\underline{748,000}$ & $\underline{845,660}$ & $\underline{2,240,890}$ & & & \\
\hline \multicolumn{2}{|c|}{ Total Weights } & $\underline{748,000}$ & $1,375,660$ & $3,035,890$ & & & \\
\hline
\end{tabular}




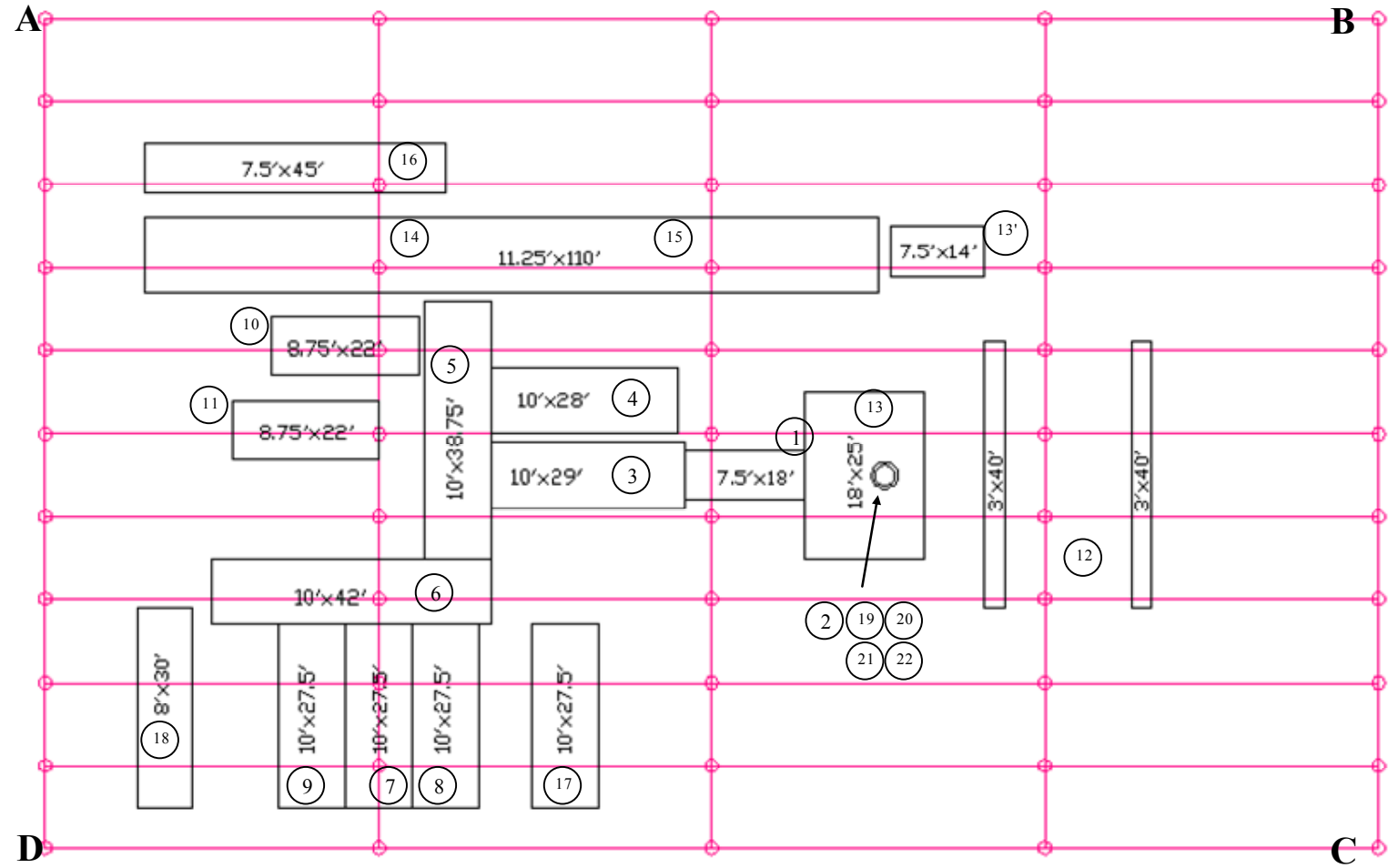

Figure 4-4. Rapid Rig layout

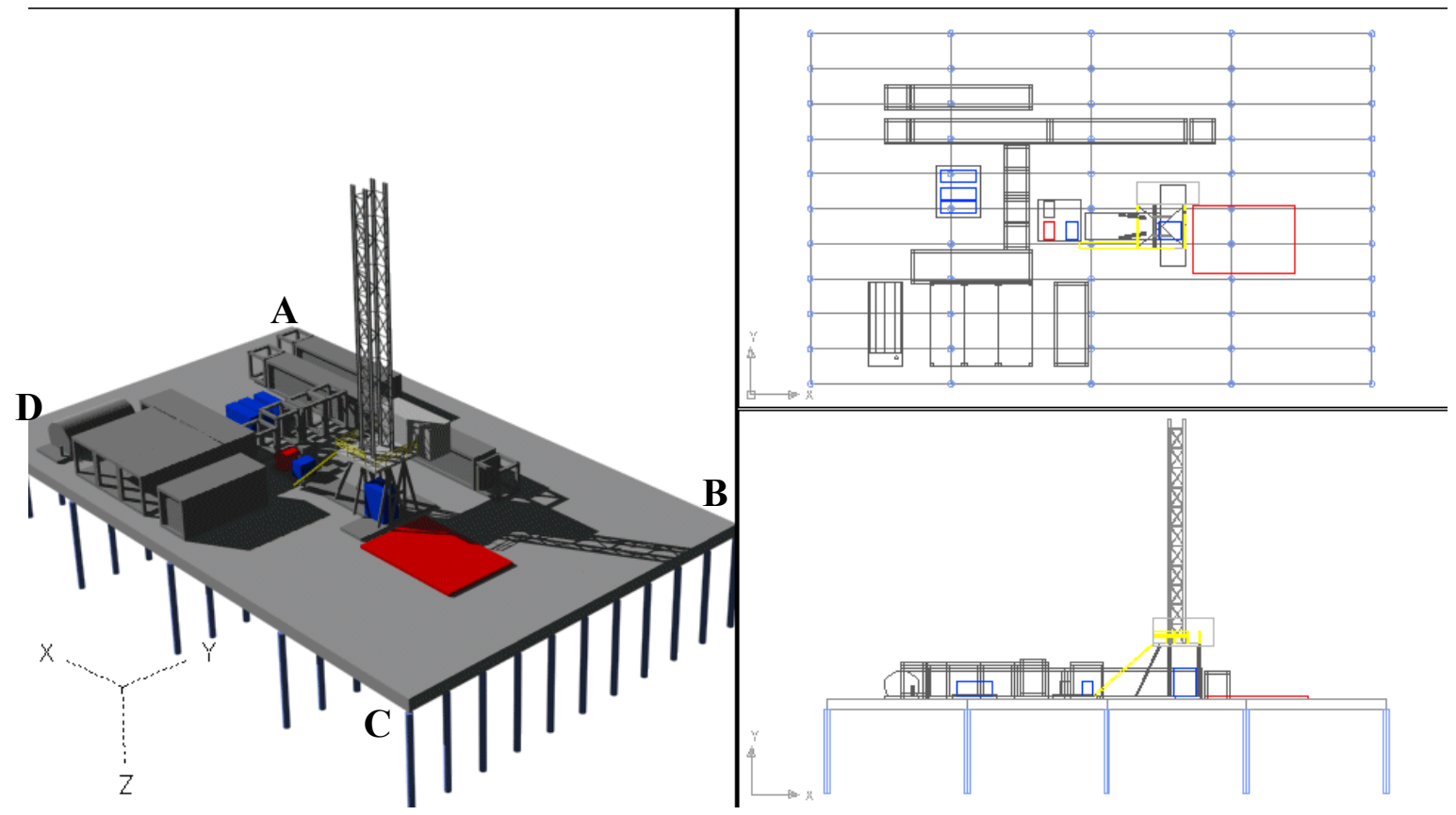

Figure 4-5. Three dimensional (3-D) layout of Rapid Rig 


\subsubsection{Soil Conditions}

The six soil conditions adopted for the general foundation calculations are used for the Rapid Rig foundation calculation (i.e., very dense, medium, and very loose for sand; hard, medium, and soft for clay).

\subsubsection{Weight Distribution on the Platform}

In order to calculate the load distribution of Rapid Rig on the proposed modular platform, a numerical analysis program, VisualFEA, is used. Since the wind load in Rapid Rig is significantly smaller than that in the general case, the dead and live loads governed the design. The following assumptions are made to perform the numerical analysis for this problem:

1. Young's modulus (E) for the aluminum material of each module is $1.44 \mathrm{E}+09 \mathrm{psf}$.

2. The modules are in the form of upside down aluminum boxes. The deck of these modules is 6 inches thick. In order to simplify the mesh generation for the numerical simulations, the modules are modeled as flat plates (called thin shells in Finite Element Analysis) which are 6 inches thick. This is a conservative assumption since it ignores the stiffness benefit derived from the $4 \mathrm{ft}$ thickness of the side beams (Figure 4-1).

3. Self weight of modules is not considered in this analysis.

4. Rigid boundary conditions are adopted (The supports of the platform do not settle). Four node quadrilateral elements are used in this analysis and the applied load layout of Rapid Rig is shown in Figure 4-6. 


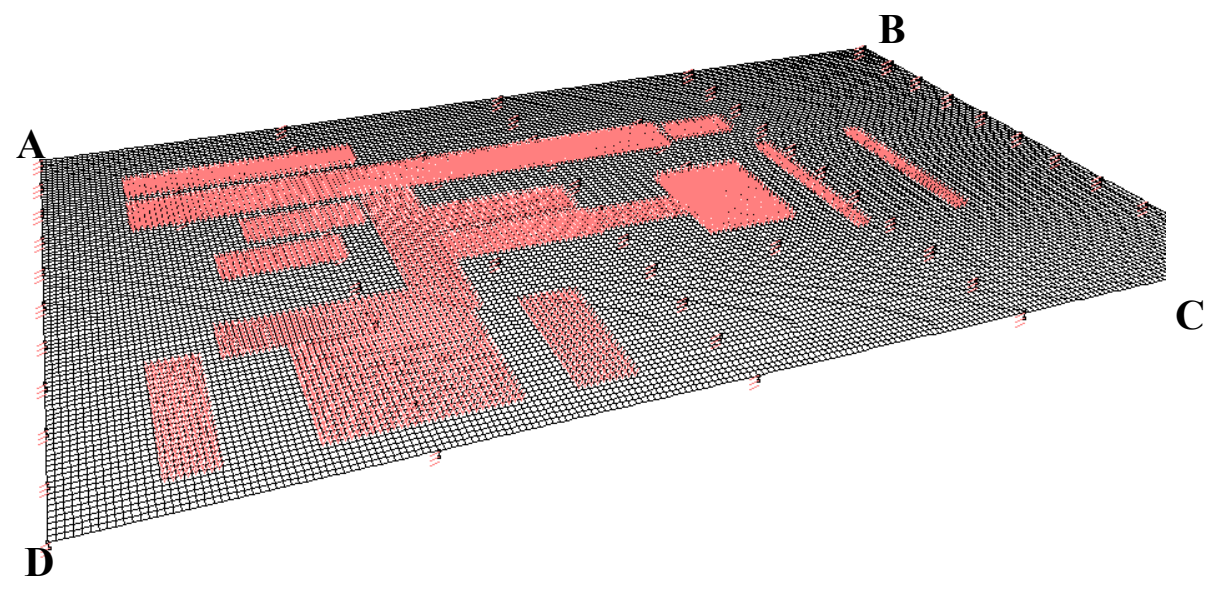

Figure 4-6. The applied load layout of Rapid Rig on the proposed platform

The pile reaction forces and the deformation of the deck on the modules are calculated by using only four piles for each module as shown in Figures 4-7 4-9. According to the results, the most critical pile reaction force and deformation are 208.3 kips, and $0.934 \mathrm{ft}$, respectively. Since $0.934 \mathrm{ft}$ is not an acceptable deformation, several critical modules are required to have six piles, each. The results of the analysis using six piles for critical modules are shown in Figures 4-10 4-12.

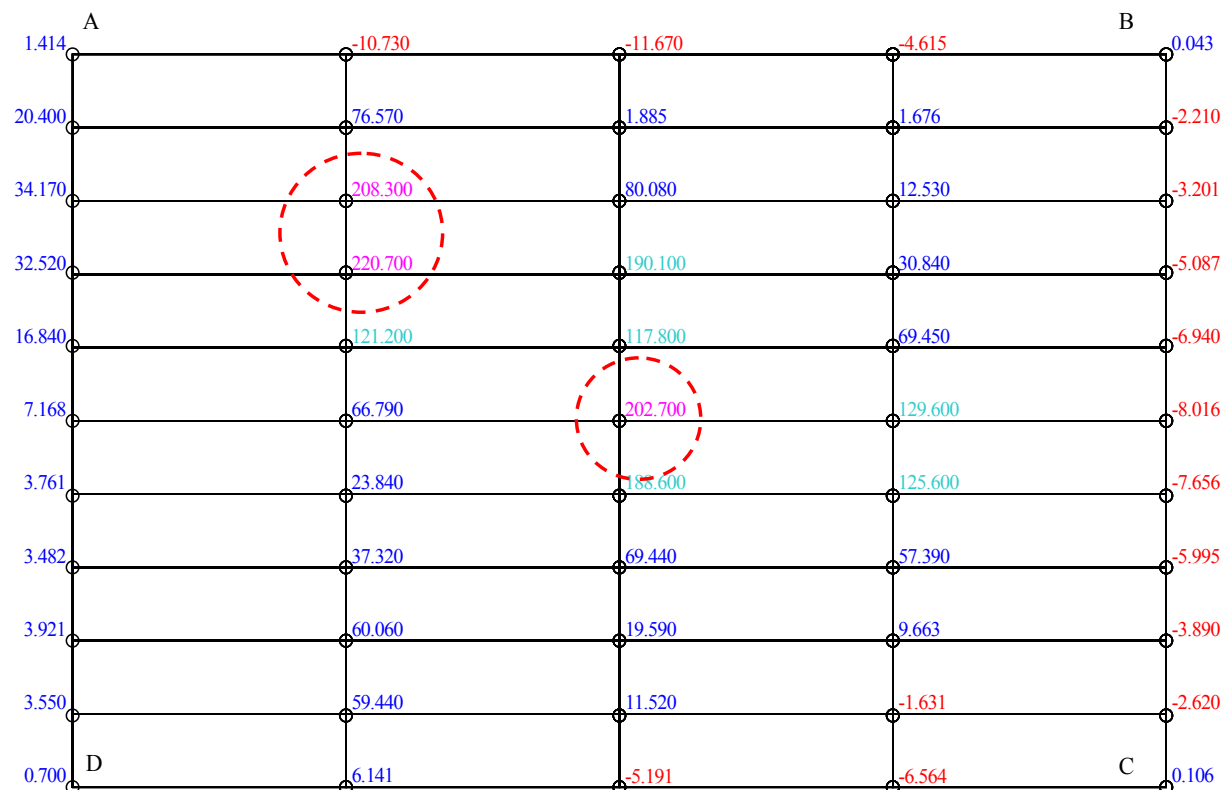

Figure 4-7. Pile reaction force [kips], (using only four piles per module) 


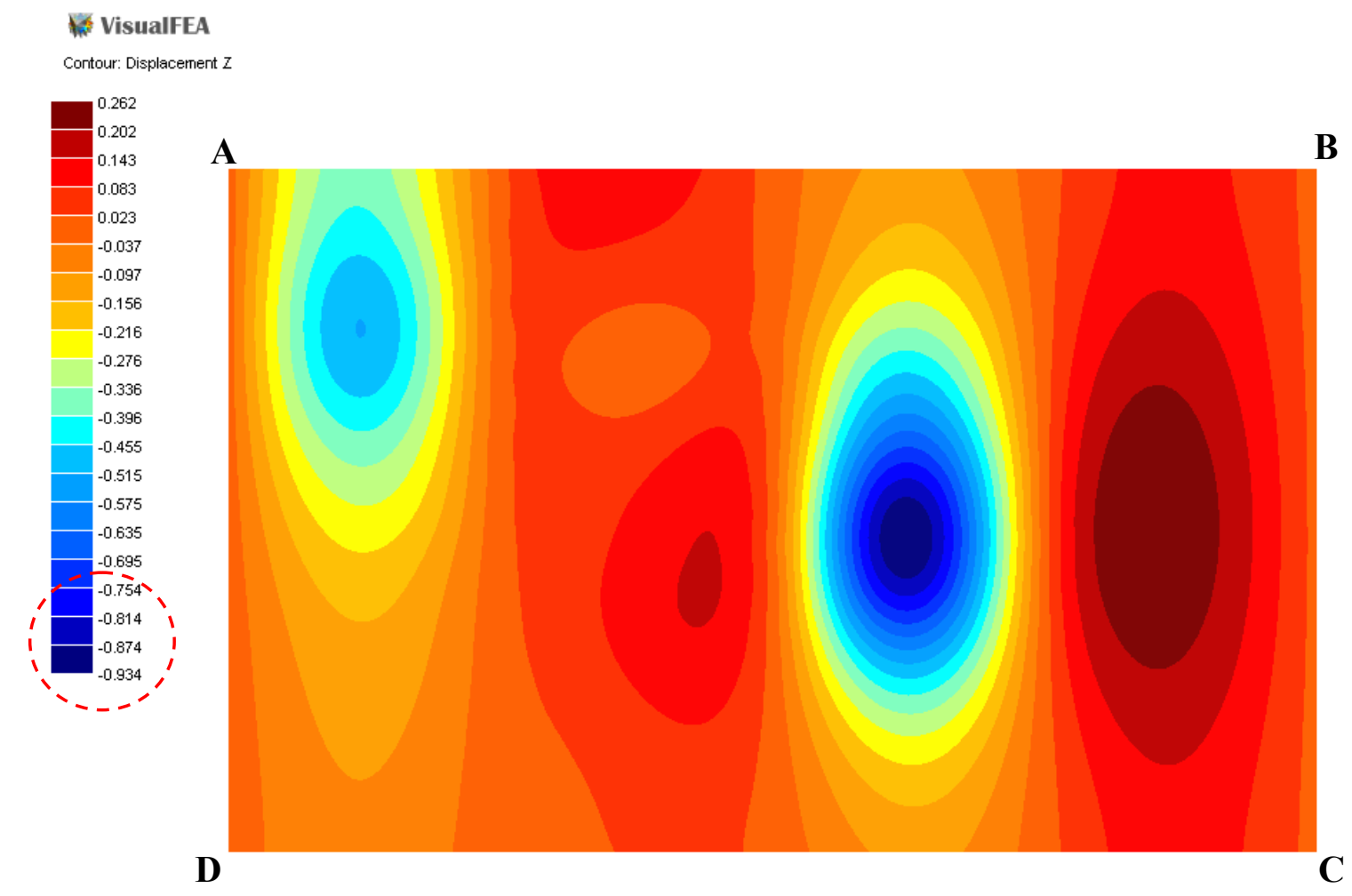

Figure 4-8. Module deformation [ft], (using only four piles per module)

\section{W VisualFEA}

Contour: Displacement $Z$
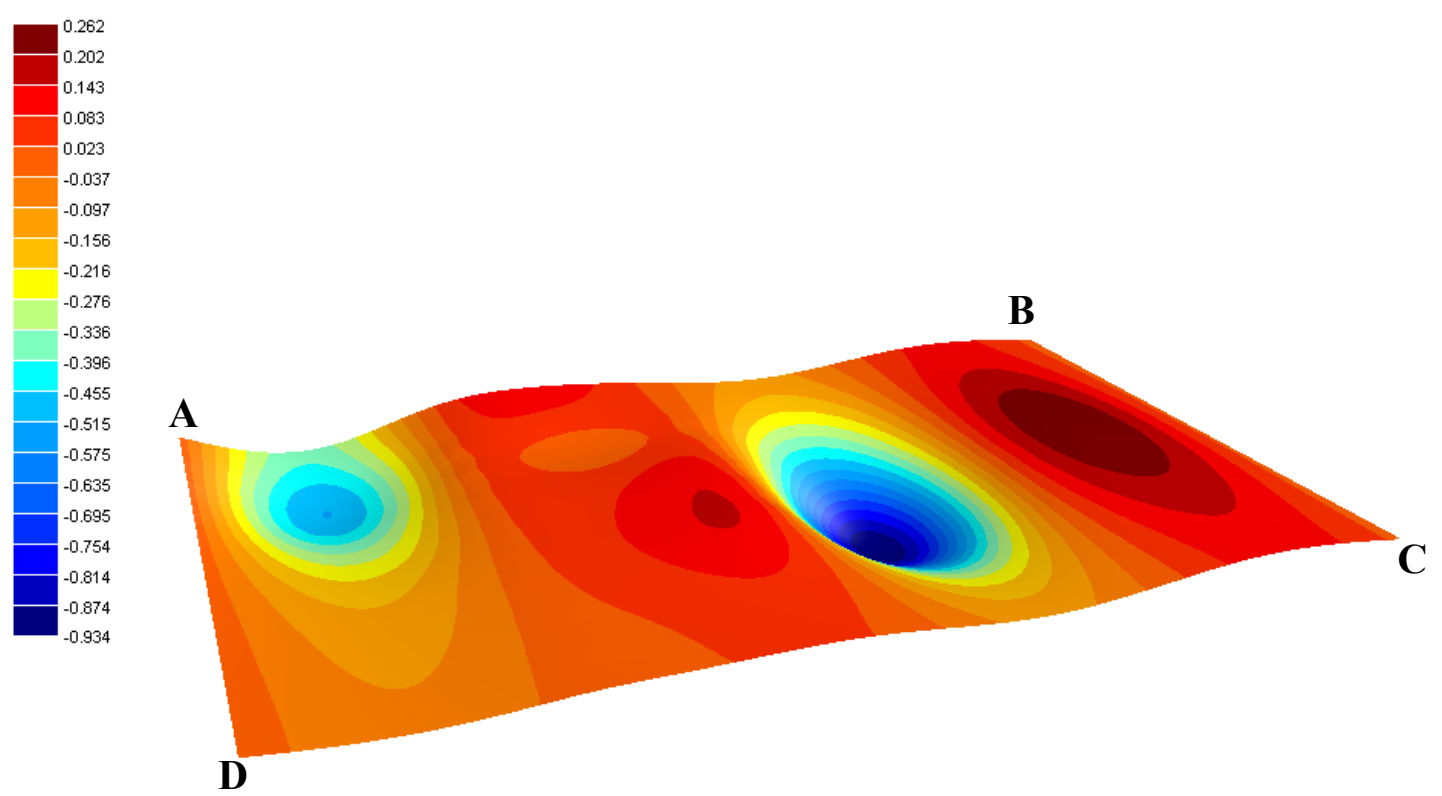

Figure 4-9. Module deformed shape (using only four piles per module) 


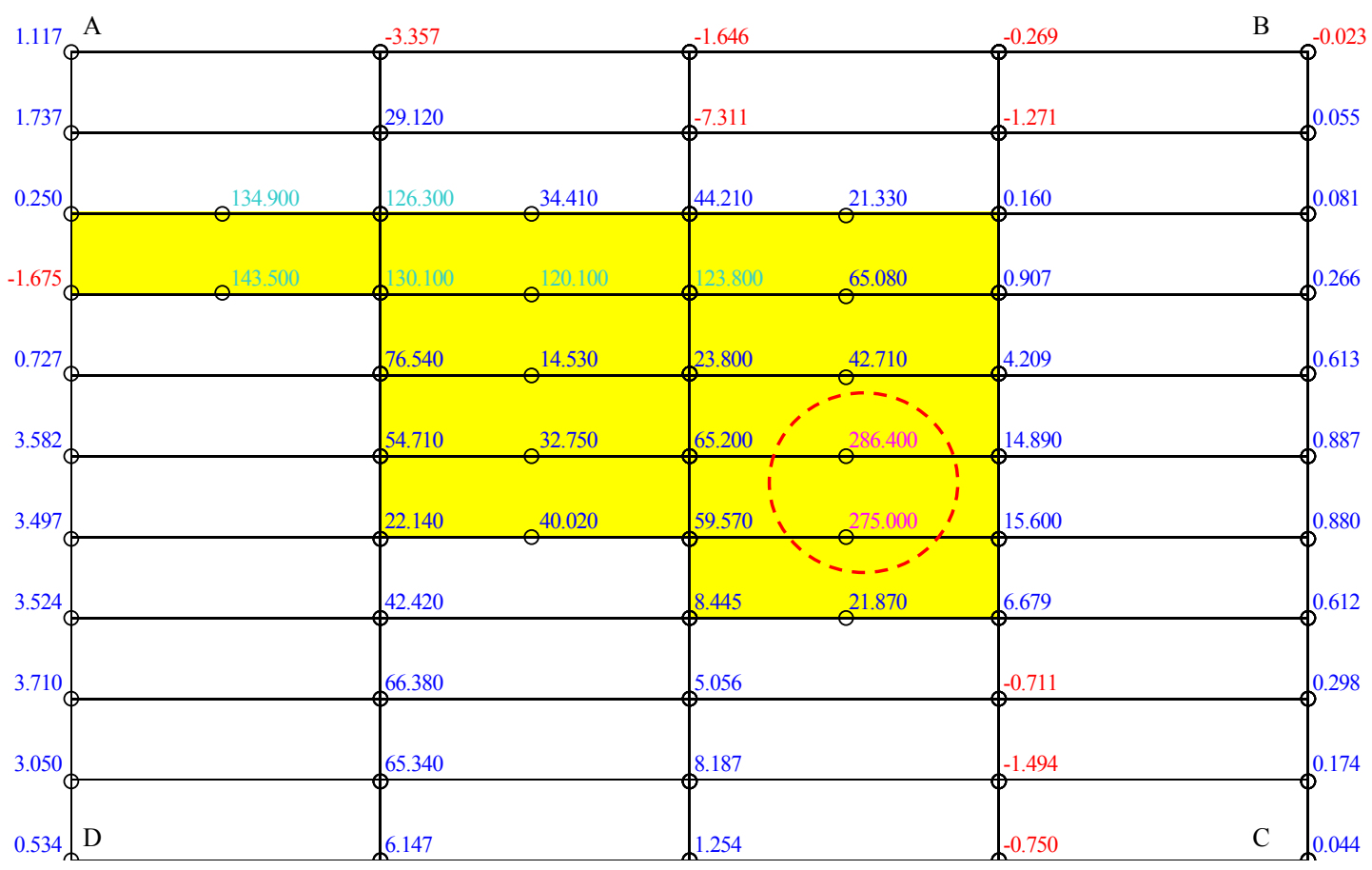

Figure 4-10. Pile reaction force [kips], (using six piles for critical modules)

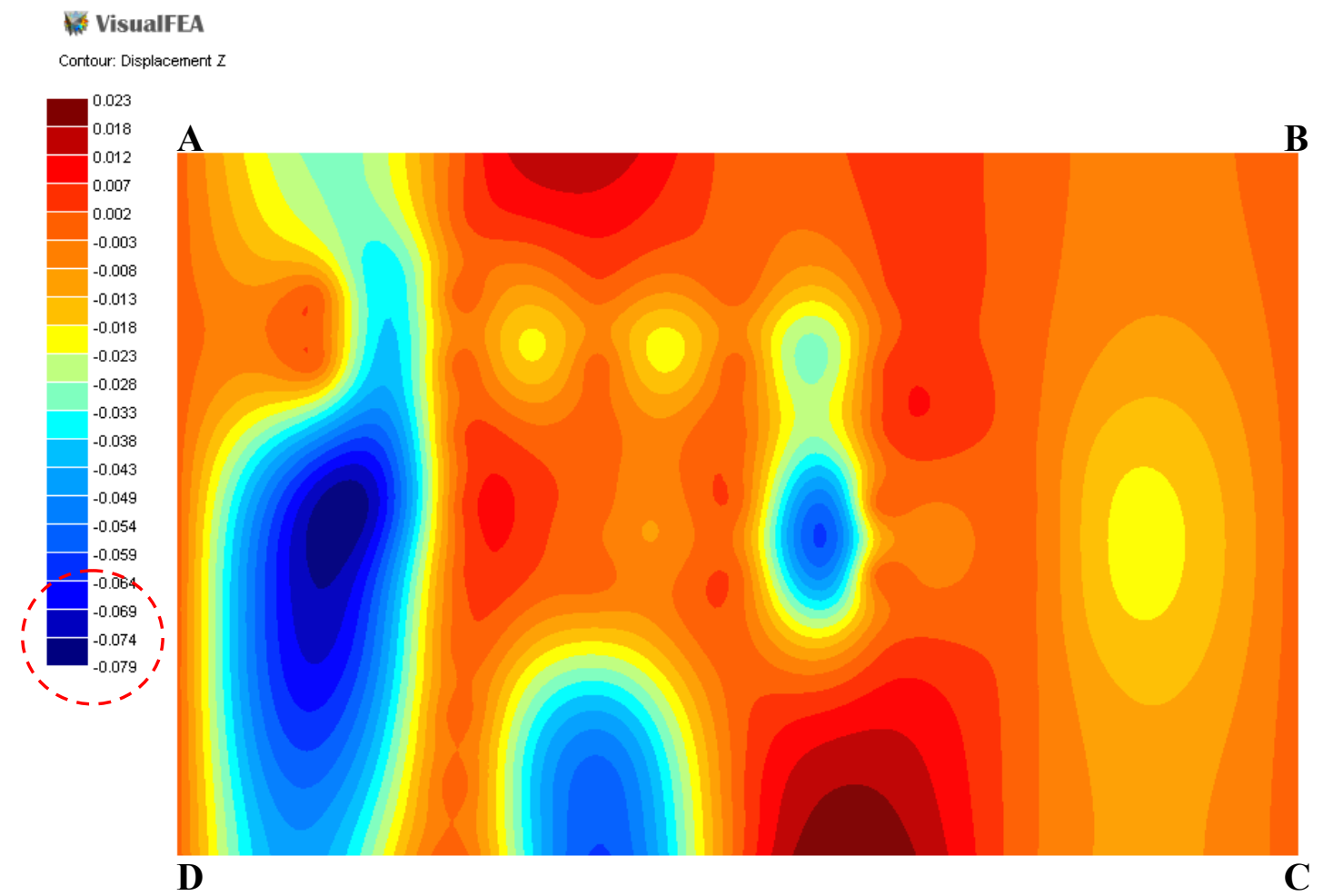

Figure 4-11. Module deformation [ft], (using six piles for critical modules) 


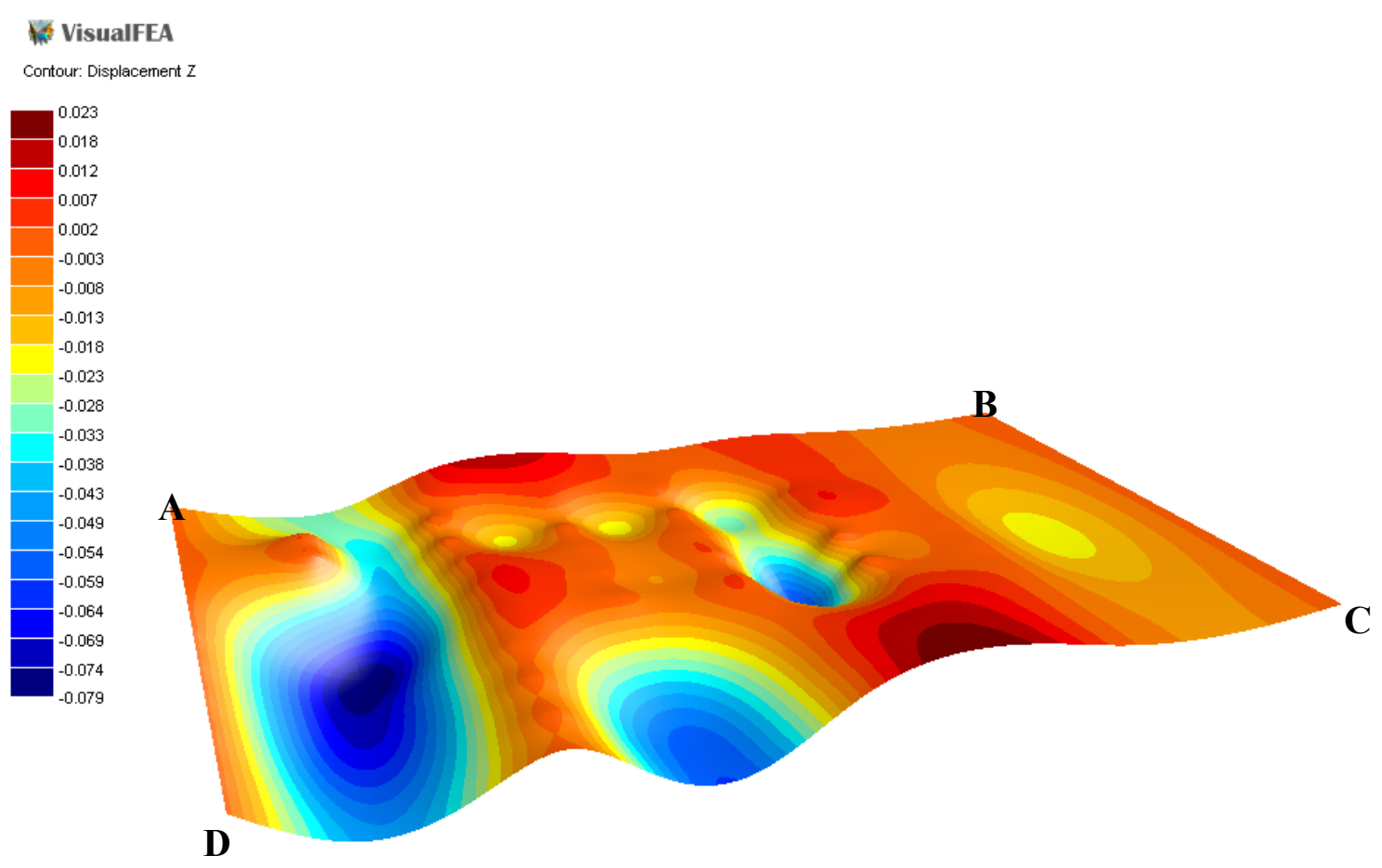

Figure 4-12. Module deformed shape (using six piles for critical modules)

\subsubsection{LRFD Method}

In the case of Rapid Rig, the pile capacity calculations are performed for the driven steel pipe piles and bored piles in accordance with the LRFD method. The maximum reaction force obtained from the Finite Element simulations as described in Section 4.2.2.2 is considered as an applied load for generating the recommended pile selection tables in the Rapid Rig case similar to the tables in the general case. Since this force is the maximum value over the platform, it is conservative to choose one size of pile based on the maximum force and to apply it for all other piles.

As can be seen in Figure 4-10, the reaction forces on most piles besides the two piles right underneath the mast are significantly lower than the maximum reaction value. Therefore, if a single size of pile is used for the whole area, it is not an economical design. Instead, Table 4-3 and 4-6 developed in the general case can be used to choose a proper size for those piles subjected to relatively low reaction forces. 


\subsubsection{Results Summary}

Based on the pile capacity calculations in the case of Rapid Rig, two tables (Table 4-8 4-9) are developed to provide a simple way to choose an appropriate pile size for various soil conditions according to the LRFD method.

Table 4-8. Recommended size of driven piles in the Rapid Rig case (LRFD)

\begin{tabular}{|c|c|c|c|c|c|c|c|}
\hline \multirow{3}{*}{$\begin{array}{l}\text { Weight of } \\
\text { Rigs \& } \\
\text { Accessories } \\
\text { (Unfactored) }\end{array}$} & \multirow{3}{*}{$\begin{array}{l}\text { Soil types } \\
\text { ored } \\
\text { vertical } \\
\text { s on one pild }\end{array}$} & \multicolumn{3}{|c|}{ Sand \& Gravels } & \multicolumn{3}{|c|}{ Silts \& Clays } \\
\hline & & very loose & medium & very dense & soft & medium & hard \\
\hline & & $\begin{array}{l}\gamma_{\mathrm{sat}}=115 \mathrm{pcf} \\
\text { G.W.L }=0 \mathrm{ft} \\
\mathrm{N}_{\mathrm{SPT}}=10 \mathrm{bpf}\end{array}$ & $\begin{array}{l}\gamma_{\mathrm{sat}}=120 \mathrm{pcf} \\
\text { G.W.L }=10 \mathrm{ft} \\
\mathrm{N}_{\mathrm{SPT}}=30 \mathrm{bpf}\end{array}$ & $\begin{array}{l}\gamma_{\mathrm{sat}}=127 \mathrm{pcf} \\
\text { G.W.L }=20 \mathrm{ft} \\
\mathrm{N}_{\mathrm{SPT}}=50 \mathrm{bpf}\end{array}$ & $\begin{array}{l}\gamma_{\text {sat }}=115 \mathrm{pcf} \\
\text { G.W.L }=0 \mathrm{ft} \\
\mathrm{Su}=0.25 \mathrm{P}_{\mathrm{o}}{ }^{\prime}\end{array}$ & $\begin{array}{c}\gamma_{\mathrm{sat}}=120 \mathrm{pcf} \\
\text { G.W.L }=10 \mathrm{ft} \\
\mathrm{Su}=60 \mathrm{kPa}\end{array}$ & $\begin{array}{l}\gamma_{\mathrm{sat}}=127 \mathrm{pcf} \\
\mathrm{G} \cdot \mathrm{W} \cdot \mathrm{L}=20 \mathrm{ft} \\
\mathrm{Su}=100 \mathrm{kPa}\end{array}$ \\
\hline \multirow{2}{*}{594 kips } & \multirow{2}{*}{286.4 kips } & $\mathrm{D}=24 \mathrm{in}$ & $\mathrm{D}=24 \mathrm{in}$. & $\mathrm{D}=20 \mathrm{in}$. & $\mathrm{D}=24 \mathrm{in}$. & $\mathrm{D}=24 \mathrm{in}$. & $\mathrm{D}=24 \mathrm{in}$. \\
\hline & & $\mathrm{L}=84 \mathrm{ft}$ & $\mathrm{L}=43 \mathrm{ft}$ & $\mathrm{L}=23 \mathrm{ft}$ & $\mathrm{L}=96 \mathrm{ft}$ & $\mathrm{L}=68 \mathrm{ft}$ & $\mathrm{L}=48 \mathrm{ft}$ \\
\hline
\end{tabular}

Table 4-9. Recommended size of bored piles in the Rapid Rig case (LRFD)

\begin{tabular}{|c|c|c|c|c|c|c|c|}
\hline \multirow{3}{*}{$\begin{array}{l}\text { Weight of } \\
\text { Rigs \& } \\
\text { Accessories } \\
\text { (Unfactored) }\end{array}$} & \multirow{3}{*}{$\begin{array}{l}\text { Soil types } \\
\text { ored } \\
\text { vertical } \\
\text { on one pile }\end{array}$} & \multicolumn{3}{|c|}{ Sand \& Gravels } & \multicolumn{3}{|c|}{ Silts \& Clays } \\
\hline & & very loose & medium & very dense & soft & medium & hard \\
\hline & & $\begin{array}{c}\gamma_{\mathrm{sat}}=115 \mathrm{pcf} \\
\text { G.W.L }=0 \mathrm{ft} \\
\text { NSPT }=10 \mathrm{bpf}\end{array}$ & $\begin{array}{l}\gamma_{\mathrm{sat}}=120 \mathrm{pcf} \\
\text { G.W.L }=10 \mathrm{ft} \\
\text { NsPT }=30 \mathrm{bpf}\end{array}$ & $\begin{array}{l}\gamma_{\mathrm{sat}}=127 \mathrm{pcf} \\
\text { G.W.L }=20 \mathrm{ft} \\
\text { Nsp }=50 \mathrm{bpf}\end{array}$ & $\begin{array}{l}\gamma_{\mathrm{sat}}=115 \mathrm{pcf} \\
\mathrm{G} . \mathrm{W} . \mathrm{L}=0 \mathrm{ft} \\
\mathrm{Su}=0.25 \mathrm{P}_{\mathrm{o}}^{\prime}\end{array}$ & $\begin{array}{c}\gamma_{\mathrm{sat}}=120 \mathrm{pcf} \\
\mathrm{G} . \mathrm{W} . \mathrm{L}=10 \mathrm{ft} \\
\mathrm{Su}=60 \mathrm{kPa}\end{array}$ & $\begin{array}{l}\gamma_{\mathrm{sat}}=127 \mathrm{pcf} \\
\mathrm{G} \cdot \mathrm{W} \cdot \mathrm{L}=20 \mathrm{ft} \\
\mathrm{Su}=100 \mathrm{kPa}\end{array}$ \\
\hline \multirow{2}{*}{1594 kips } & \multirow{2}{*}{286.4 kips } & $\mathrm{D}=24 \mathrm{in}$ & $\mathrm{D}=24 \mathrm{in}$ & $\mathrm{D}=20 \mathrm{in}$ & $\mathrm{D}=24 \mathrm{in}$ & $\mathrm{D}=24 \mathrm{in}$ & $\mathrm{D}=24 \mathrm{in}$. \\
\hline & & $\mathrm{L}=75 \mathrm{ft}$ & $\mathrm{L}=40 \mathrm{ft}$ & $\mathrm{L}=36 \mathrm{ft}$ & $\mathrm{L}=137 \mathrm{ft}$ & $\mathrm{L}=103 \mathrm{ft}$ & $\mathrm{L}=62 \mathrm{ft}$ \\
\hline
\end{tabular}

(where, $\mathrm{P}_{\mathrm{o}}{ }^{\prime}=$ effective overburden pressure, G.W.L. = ground water depth measured from the ground surface, 


\subsubsection{Description of an Elevated Platform with Rapid Rig and a Wind Turbine}

Generation of power for drilling and production operations by wind is a feasible approach in environmentally sensitive areas. The total vertical load of a wind turbine manufactured by Made, (a Spanish company with a specialty in wind and solar technology), is used for the foundation calculation of the proposed modular platform. Technical characteristics of the wind turbine chosen for this calculation are shown in Table 4-10. The load breakdown and the layout of Rapid Rig with the wind turbine are shown in Table 4-11, Table 4-12, Figure 4-13, and Figure 4-14, respectively. Since the wind load is considerably high for the wind turbine, the operating environmental condition and extreme environmental condition are both considered in this foundation calculation.

Table 4-10. Specification of the wind turbine

\begin{tabular}{|c|l|l|}
\hline \multirow{4}{*}{ Rotor } & Rated power & $660 \mathrm{~kW}$ \\
\cline { 2 - 3 } & Rotor diameter & $46 \mathrm{~m}$ \\
\cline { 2 - 3 } & Power control & $1662 \mathrm{~m}^{2}$ \\
\cline { 2 - 3 } & Yaw system & Upwind, active \\
\cline { 2 - 3 } & Rotor swept area & $1662 \mathrm{~m} 2$ \\
\cline { 2 - 3 } & Number of blades & 3 \\
\cline { 2 - 3 } & Blade type & LM 21 \\
\cline { 2 - 3 } & Rotor speed & $25,5 / 17 \mathrm{rpm}$ \\
\cline { 2 - 3 } & Hub height & $45 \mathrm{~m}$ \\
\cline { 2 - 3 } & Tilt angle & $5^{\circ}$ \\
\hline \multirow{3}{*}{ Weights } & Rotor & $12.000 \mathrm{~kg}$ \\
\cline { 2 - 3 } & Nacelle (without rotor) & $25.000 \mathrm{~kg}$ \\
\cline { 2 - 3 } & Tower & $40.000 \mathrm{~kg}(43.5 \mathrm{~m})$ \\
\cline { 2 - 3 } & Total weight & $70.000 \mathrm{~kg}(43.5 \mathrm{tower})$ \\
\hline
\end{tabular}

[Source: http://www.made.es/06/english/html/ae_46.html] 
Table 4-11. Load breakdown of Rapid Rig with the wind turbine in operating condition

\begin{tabular}{|c|c|c|c|c|c|c|c|}
\hline \multirow{3}{*}{ No. } & \multirow{3}{*}{ COMPONENTS } & \multirow{3}{*}{$\begin{array}{c}\text { WEIGHTS [lbs] } \\
\text { (DEAD) }\end{array}$} & \multirow{3}{*}{$\begin{array}{c}\text { WEIGHTS [lbs] } \\
\text { (LIVE) }\end{array}$} & $\begin{array}{c}\text { Factored } \\
\text { Weights [lbs] }\end{array}$ & \multicolumn{2}{|c|}{ Dimension } & \multirow{3}{*}{ Notes } \\
\hline & & & & $D . L=1.3$ & \multirow{2}{*}{ W } & \multirow{2}{*}{$\mathrm{L}$} & \\
\hline & & & & L.L $=1.5$ & & & \\
\hline 1. & Substructure/Drillfloor package & 80,000 & & 104,000 & 10 & 58.5 & \\
\hline 2. & Mast including installed equipment & 100,000 & & 130,000 & 18 & 25 & \\
\hline 3. & Drawworks package includes Accumulator unit & 70,000 & & 91,000 & 10 & 29 & \\
\hline 4. & Utilities Skid & 25,000 & & 32,500 & 10 & 28 & \\
\hline 5. & Service Skid & 20,000 & & 26,000 & 10 & 38.75 & \\
\hline 6. & Electrical Control House & 30,000 & & 39,000 & 10 & 42 & \\
\hline 7. & Generator House \#1 & 40,000 & & 52,000 & 10 & 27.5 & \\
\hline 8. & Generator House \#2 & 40,000 & & 52,000 & 10 & 27.5 & \\
\hline 9. & Air Compressor House & 30,000 & & 39,000 & 10 & 27.5 & \\
\hline 10. & Mud Pump \#1 & 55,000 & & 71,500 & 8.75 & 22 & \\
\hline 11. & Mud Pump \#2 & 55,000 & & 71,500 & 8.75 & 22 & \\
\hline 12. & Pipe Handling equipment & 35,000 & & 45,500 & 3 & 80 & \\
\hline \multirow{2}{*}{13.} & Control House skid including choke manifold & 23,000 & & 29,900 & 18 & 12.5 & \\
\hline & Choke Manifold hauled on same trailer & 15,000 & & 19,500 & 7.5 & 14 & \\
\hline \multirow{2}{*}{14.} & Mud Tank Skid \#1 (Empty) & 40,000 & & 52,000 & 11.25 & 55 & \\
\hline & Mud Tank Skid \#1 (Full) & & 204,750 & 307,125 & 11.25 & 55 & 375 barrels, 13 lbs/gal \\
\hline \multirow{2}{*}{15.} & Mud Tank Skid \#2 (Empty) & 40,000 & & 52,000 & 11.25 & 55 & \\
\hline & Mud Tank Skid \#2 (Full) & & 204,750 & 307,125 & 11.25 & 55 & 375 barrels, $13 \mathrm{lbs} / \mathrm{gal}$ \\
\hline \multirow{2}{*}{16.} & Water Tank (Empty) & 20,000 & & 26,000 & 7.5 & 45 & \\
\hline & Water Tank (Full) & & 139,440 & 209,160 & 7.5 & 45 & 400 barrels, $8.3 \mathrm{lbs} / \mathrm{gal}$ \\
\hline 17. & Work shop/Storage Skid & 20,000 & & 26,000 & 10 & 27.5 & \\
\hline 18. & Fuel Tank Skid & 10,000 & & 13,000 & 8 & 30 & \\
\hline 19. & Casing & & 530,000 & 795,000 & 18 & 25 & $53 \mathrm{lbs} / \mathrm{ft}, 10000 \mathrm{ft}$ \\
\hline 20. & Pipes & & 234,000 & 351,000 & 18 & 25 & $19.5 \mathrm{lbs} / \mathrm{ft}, 12000 \mathrm{ft}$ \\
\hline 21. & Collars & & 2,720 & 4,080 & 18 & 25 & $80 \mathrm{lbs} / \mathrm{ft}, 34 \mathrm{ft}$ \\
\hline 22. & Drill collars & & 60,000 & 90,000 & 18 & 25 & 6000 lbs $\times 10$ \\
\hline 23. & Wind turbine $(500 \mathrm{Kw})$ & 154,000 & & 200,200 & 36 & 36 & \\
\hline \multicolumn{8}{|l|}{24.} \\
\hline \multicolumn{2}{|c|}{ Total Weights without Casing } & $\underline{902,000}$ & $\underline{845,660}$ & $\underline{2,441,090}$ & & & \\
\hline \multicolumn{2}{|c|}{ Total Weights } & $\underline{902,000}$ & $1,375,660$ & $\underline{3,236,090}$ & & & \\
\hline
\end{tabular}

For the operating environmental condition, the load factors of dead load, live load, and wind load are 1.3,1.5, and 1.2, respectively. 
Table 4-12. Load breakdown of Rapid Rig with the wind turbine in extreme condition

\begin{tabular}{|c|c|c|c|c|c|c|c|}
\hline \multirow{3}{*}{ No. } & \multirow{3}{*}{ COMPONENTS } & \multirow{3}{*}{$\begin{array}{c}\text { WEIGHTS [lbs] } \\
\text { (DEAD) }\end{array}$} & \multirow{3}{*}{$\begin{array}{c}\text { WEIGHTS [lbs] } \\
\text { (LIVE) }\end{array}$} & $\begin{array}{c}\text { Factored } \\
\text { Weights [lbs] }\end{array}$ & \multicolumn{2}{|c|}{ Dimension } & \multirow{3}{*}{ Notes } \\
\hline & & & & $\mathrm{D} . \mathrm{L}=1.1$ & \multirow{2}{*}{ W } & \multirow{2}{*}{ L } & \\
\hline & & & & L.L $=1.1$ & & & \\
\hline 1. & Substructure/Drillfloor package & 80,000 & & 88,000 & 10 & 58.5 & \\
\hline 2. & Mast including installed equipment & 100,000 & & 110,000 & 18 & 25 & \\
\hline 3. & Drawworks package includes Accumulator unit & 70,000 & & 77,000 & 10 & 29 & \\
\hline 4. & Utilities Skid & 25,000 & & 27,500 & 10 & 28 & \\
\hline 5. & Service Skid & 20,000 & & 22,000 & 10 & 38.75 & \\
\hline 6. & Electrical Control House & 30,000 & & 33,000 & 10 & 42 & \\
\hline 7. & Generator House \#1 & 40,000 & & 44,000 & 10 & 27.5 & \\
\hline 8. & Generator House \#2 & 40,000 & & 44,000 & 10 & 27.5 & \\
\hline 9. & Air Compressor House & 30,000 & & 33,000 & 10 & 27.5 & \\
\hline 10. & Mud Pump \#1 & 55,000 & & 60,500 & 8.75 & 22 & \\
\hline 11. & Mud Pump \#2 & 55,000 & & 60,500 & 8.75 & 22 & \\
\hline 12. & Pipe Handling equipment & 35,000 & & 38,500 & 3 & 80 & \\
\hline \multirow{2}{*}{13.} & Control House skid including choke manifold & 23,000 & & 25,300 & 18 & 12.5 & \\
\hline & Choke Manifold hauled on same trailer & 15,000 & & 16,500 & 7.5 & 14 & \\
\hline \multirow{2}{*}{14.} & Mud Tank Skid \#1 (Empty) & 40,000 & & 44,000 & 11.25 & 55 & \\
\hline & Mud Tank Skid \#1 (Full) & & 204,750 & 225,225 & 11.25 & 55 & 375 barrels, $13 \mathrm{lbs} / \mathrm{gal}$ \\
\hline \multirow{2}{*}{15.} & Mud Tank Skid \#2 (Empty) & 40,000 & & 44,000 & 11.25 & 55 & \\
\hline & Mud Tank Skid \#2 (Full) & & 204,750 & 225,225 & 11.25 & 55 & 375 barrels, 13 lbs/gal \\
\hline \multirow{2}{*}{16.} & Water Tank (Empty) & 20,000 & & 22,000 & 7.5 & 45 & \\
\hline & Water Tank (Full) & & 139,440 & 153,384 & 7.5 & 45 & 400 barrels, $8.3 \mathrm{lbs} / \mathrm{gal}$ \\
\hline 17. & Work shop/Storage Skid & 20,000 & & 22,000 & 10 & 27.5 & \\
\hline 18. & Fuel Tank Skid & 10,000 & & 11,000 & 8 & 30 & \\
\hline 19. & Casing & & 530,000 & 583,000 & 18 & 25 & $53 \mathrm{lbs} / \mathrm{ft}, 10000 \mathrm{ft}$ \\
\hline 20. & Pipes & & 234,000 & 257,400 & 18 & 25 & $19.5 \mathrm{lbs} / \mathrm{ft}, 12000 \mathrm{ft}$ \\
\hline 21. & Collars & & 2,720 & 2,992 & 18 & 25 & $80 \mathrm{lbs} / \mathrm{ft}, 34 \mathrm{ft}$ \\
\hline 22. & Drill collars & & 60,000 & 66,000 & 18 & 25 & 6000 lbs $\times 10$ \\
\hline 23. & Wind turbine $(500 \mathrm{Kw})$ & 154,000 & & 169,400 & 36 & 36 & \\
\hline \multicolumn{8}{|l|}{24.} \\
\hline \multicolumn{2}{|c|}{ Total Weights without Casing } & $\underline{902,000}$ & $\underline{845,660}$ & $1,922,426$ & & & \\
\hline \multicolumn{2}{|c|}{ Total Weights } & $\underline{902,000}$ & $\underline{1,375,660}$ & $\underline{2,505,426}$ & & & \\
\hline
\end{tabular}

For the extreme environmental condition, the load factors of dead load, live load, and wind load are $1.1,1.1$, and 1.35 , respectively. 


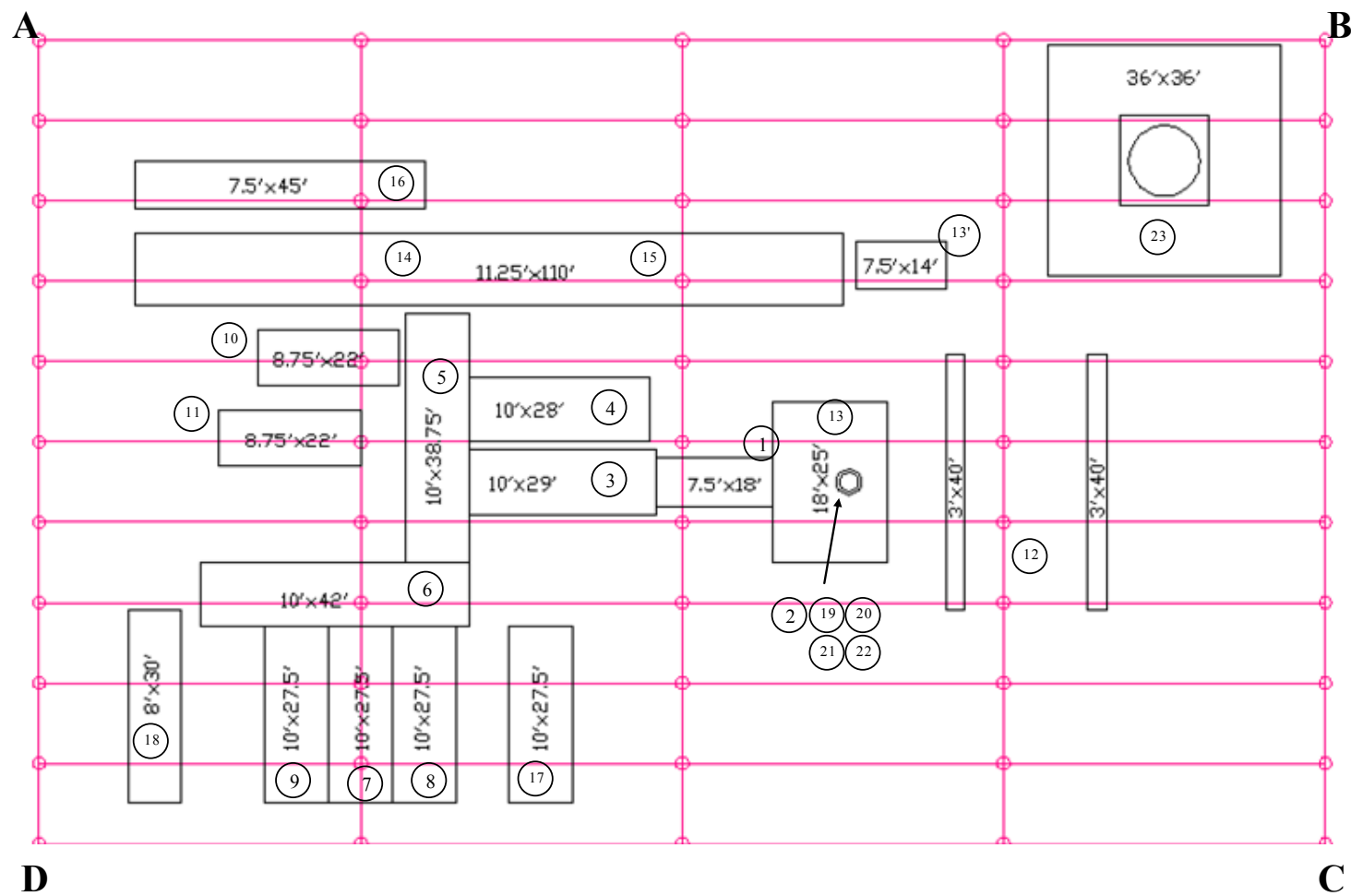

Figure 4-13. Layout of Rapid Rig and the wind turbine

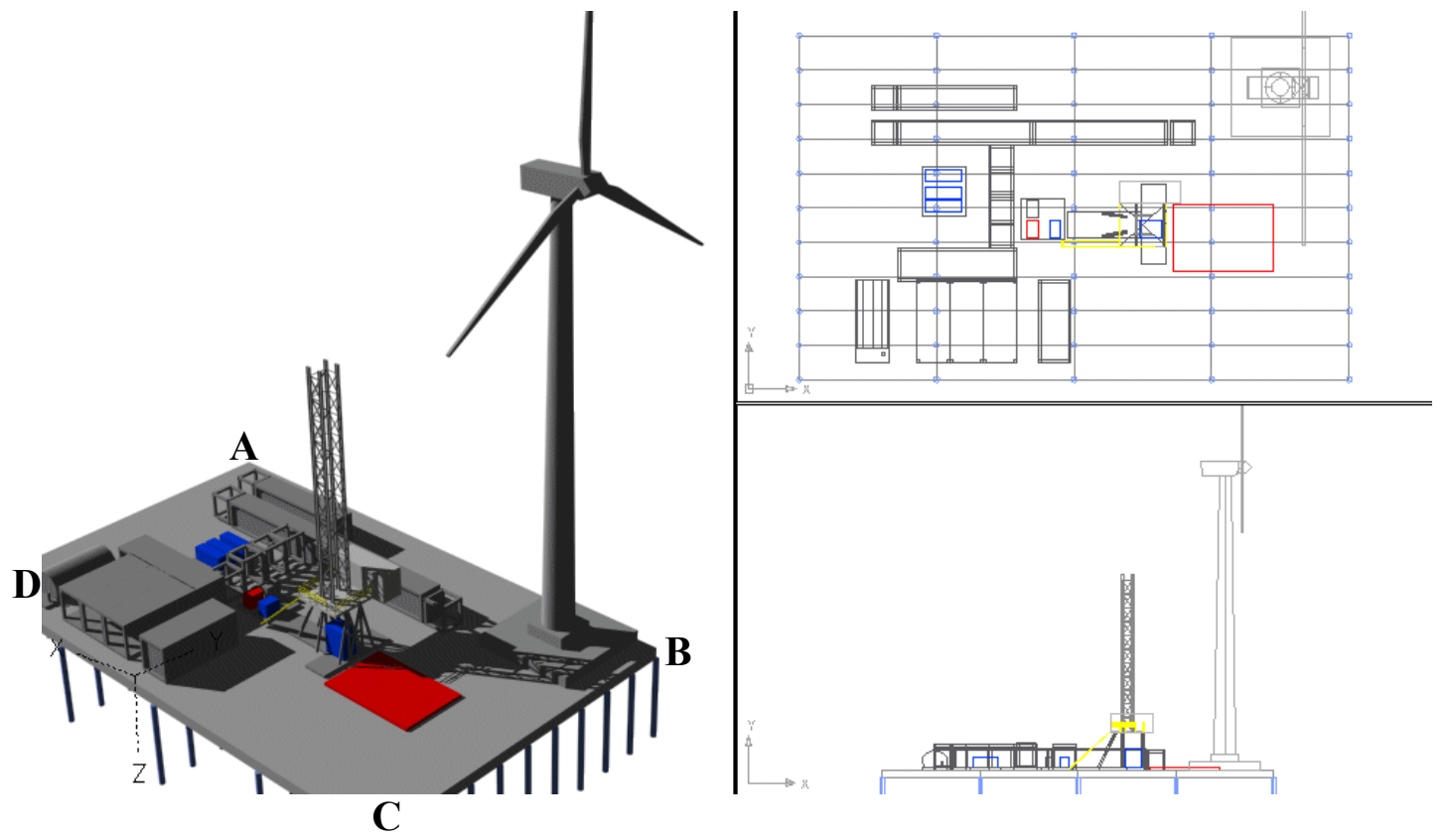

Figure 4-14. Three dimensional (3-D) layout of Rapid Rig and the wind turbine 


\subsubsection{Soil Conditions}

The six soil conditions adopted for the general foundation calculations are used for this foundation calculation (i.e., very dense, medium, very loose for sand; hard, medium, soft for clay).

\subsubsection{Weight Distribution on Platform}

In order to calculate the load distribution of Rapid Rig with the wind turbine on the proposed modular platform, a numerical analysis program, VisualFEA, is used. Since the height of the wind turbine is around $150 \mathrm{ft}$, the wind load should be considered in this analysis. Following assumptions are made to perform the numerical analysis for this problem:

1. Young's modulus (E) for the aluminum material of each module is $1.44 \mathrm{E}+09 \mathrm{psf}$.

2. The modules are represented by a 6 inches thick plate.

3. Self weight of modules is not considered in this analysis.

4. Rigid boundary conditions are adopted (The supports of the platform do not settle). Four node quadrilateral elements are used in this analysis and the applied load layout of Rapid Rig with the wind turbine is shown in Figure 4-15.

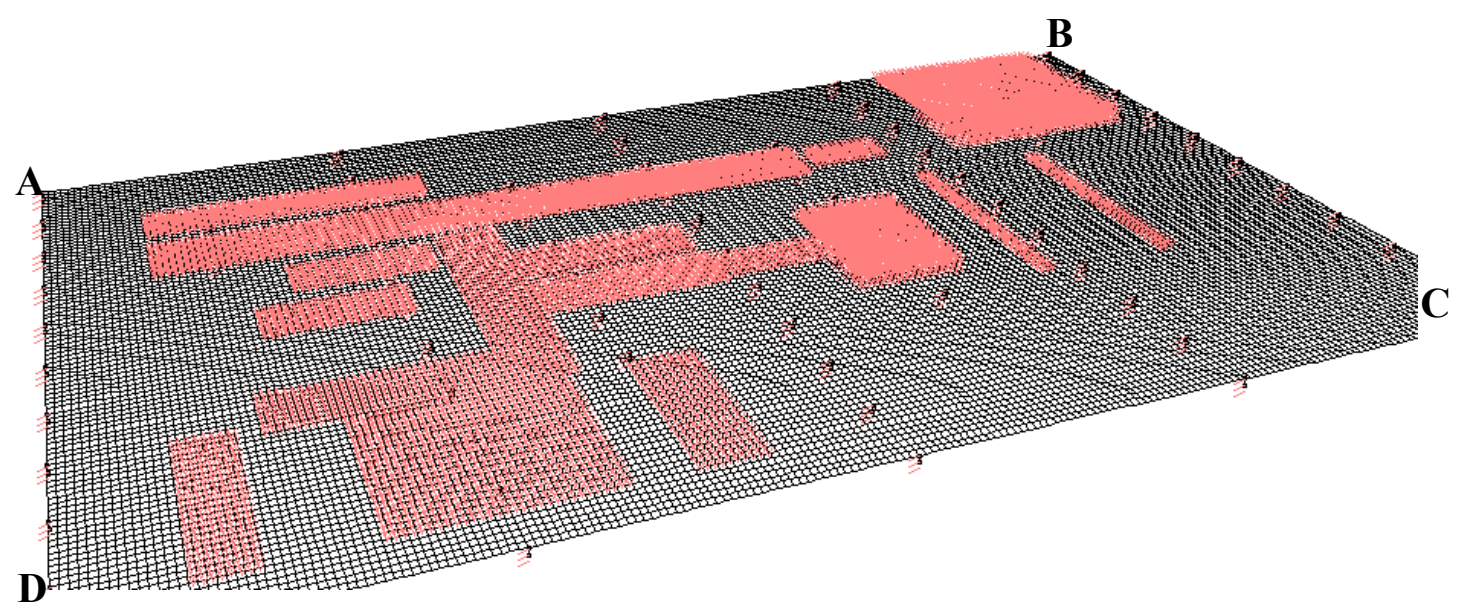

Figure 4-15. Applied load layout of Rapid Rig and the wind turbine 
The pile reaction forces and the deformation of the deck on the modules are calculated by using only four piles for each module as shown in Figures 4-16 4-21. According to the results, the most critical pile reaction force and deformation for the operating environmental condition and the extreme environmental condition are 221.7 kips, $0.909 \mathrm{ft}, 167.4 \mathrm{kips}$, and $1.054 \mathrm{ft}$, respectively. Since $1.0 \mathrm{ft}$ is not an acceptable deformation, several critical modules are required to have six piles, each. The results of the analysis using six piles for critical modules are shown in Figure 4-22 4-27.

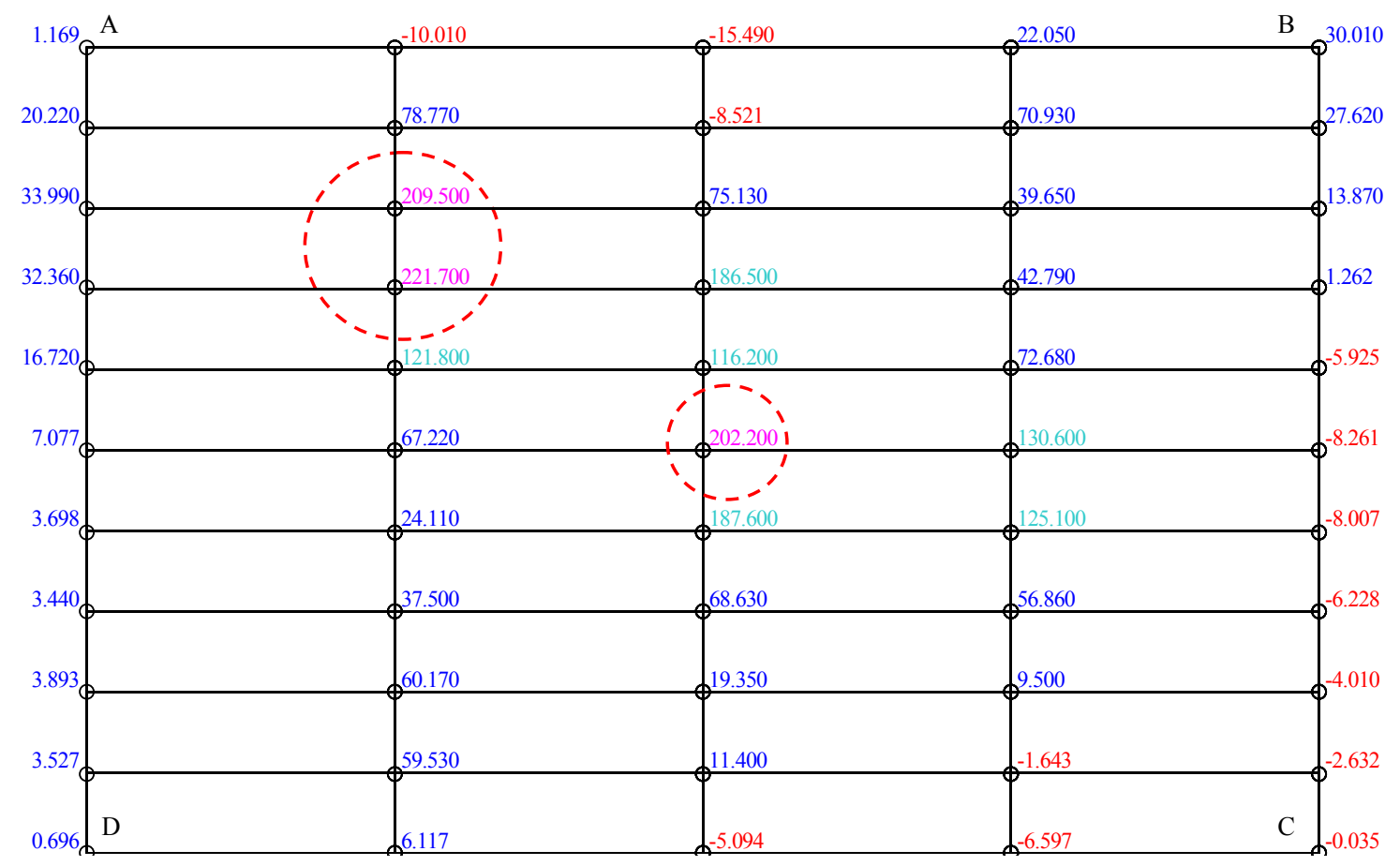

Figure 4-16. Pile reaction force [kips] in operating condition 


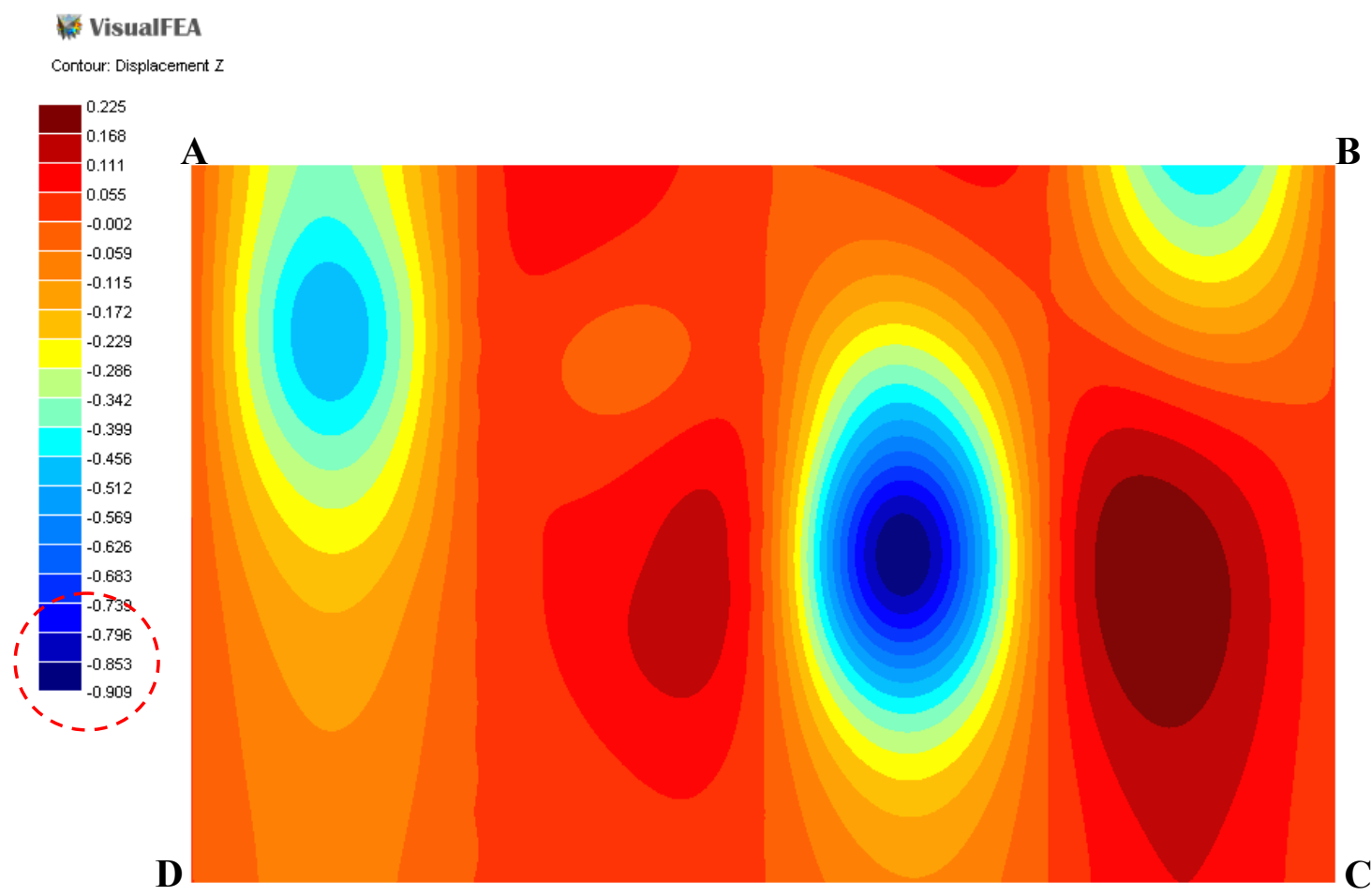

Figure 4-17. Module deformation [ft] in operating condition

\section{VisualFEA}

Contour: Displacement Z
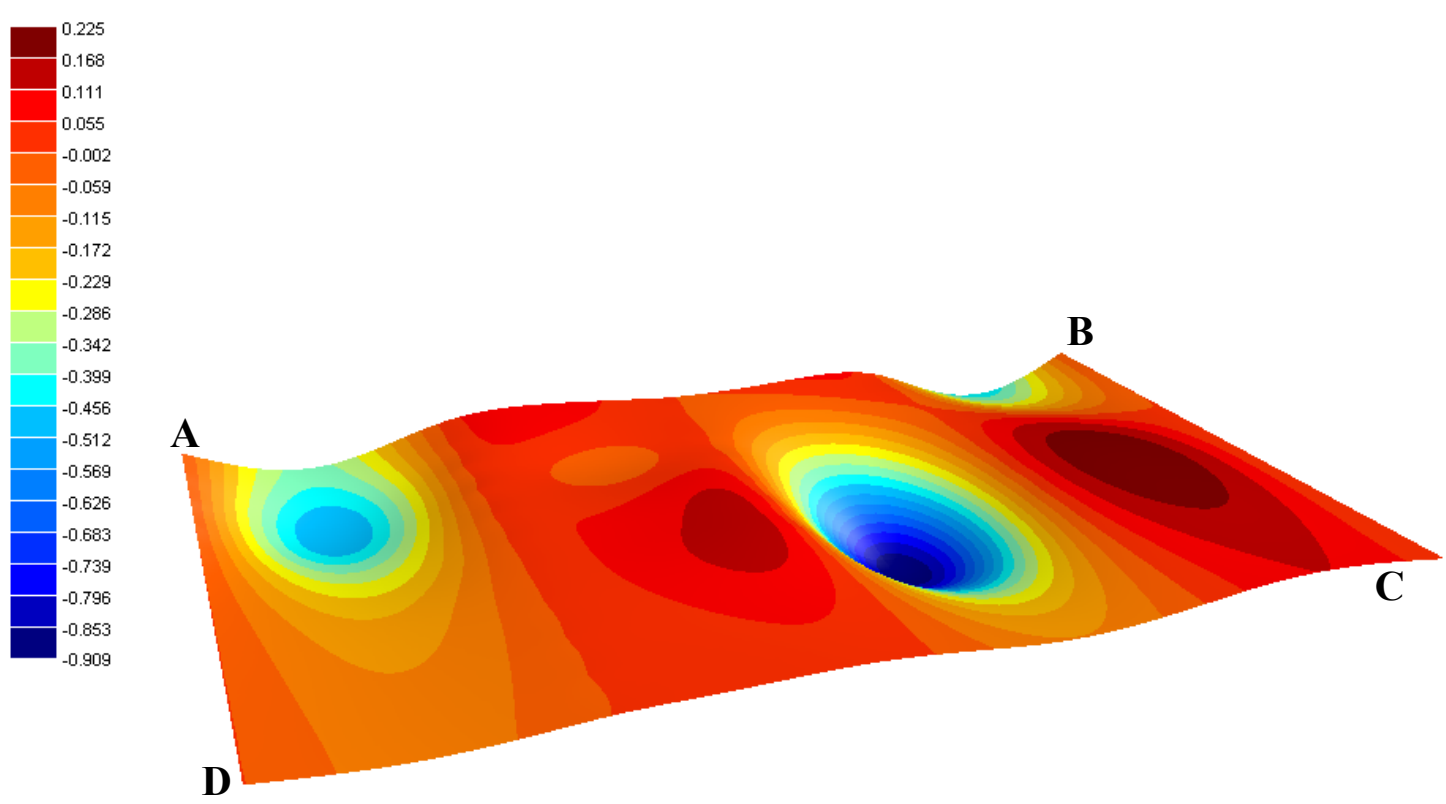

Figure 4-18. Module deformed shape in operating condition 


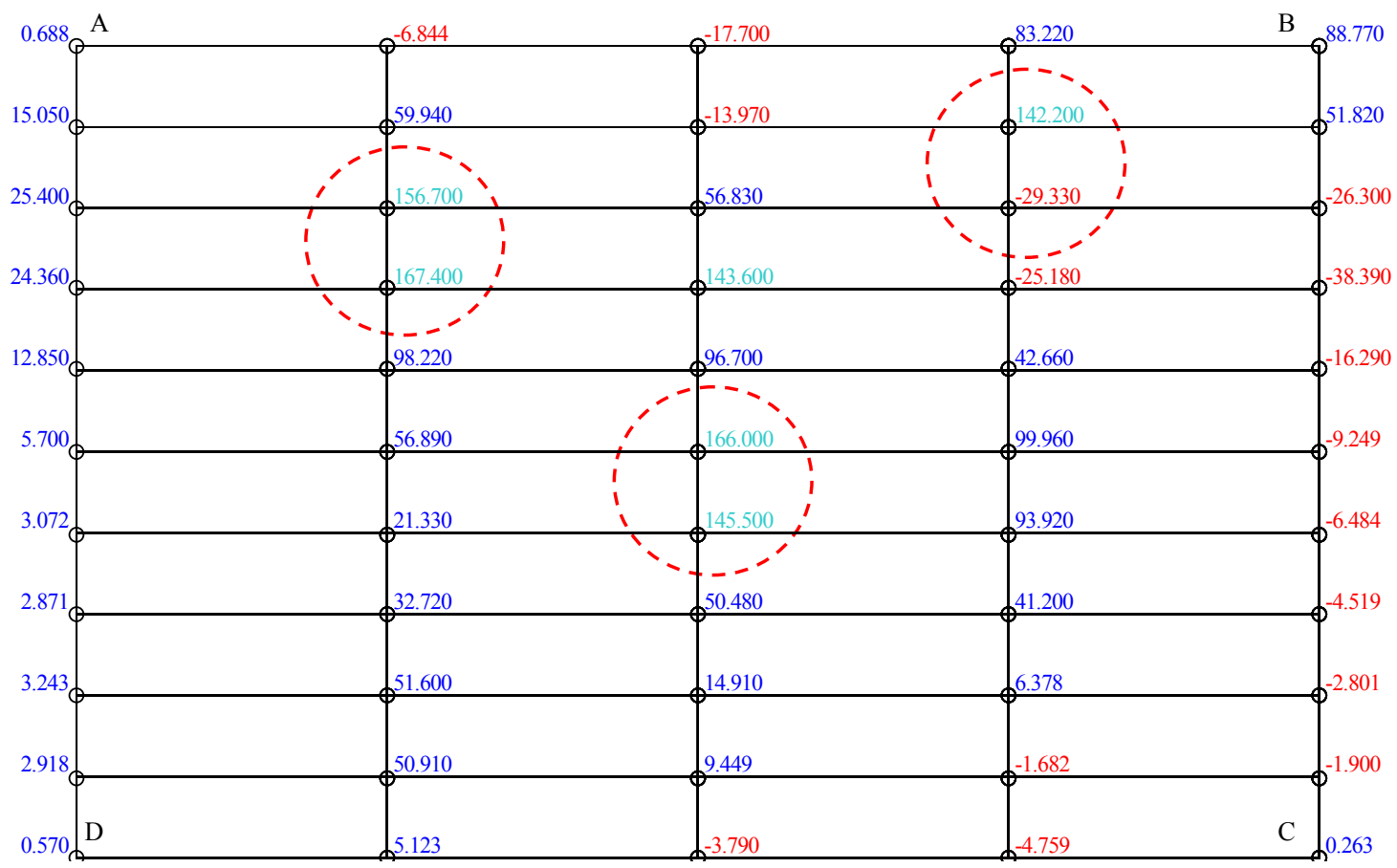

Figure 4-19. Pile reaction force [kips] in extreme condition

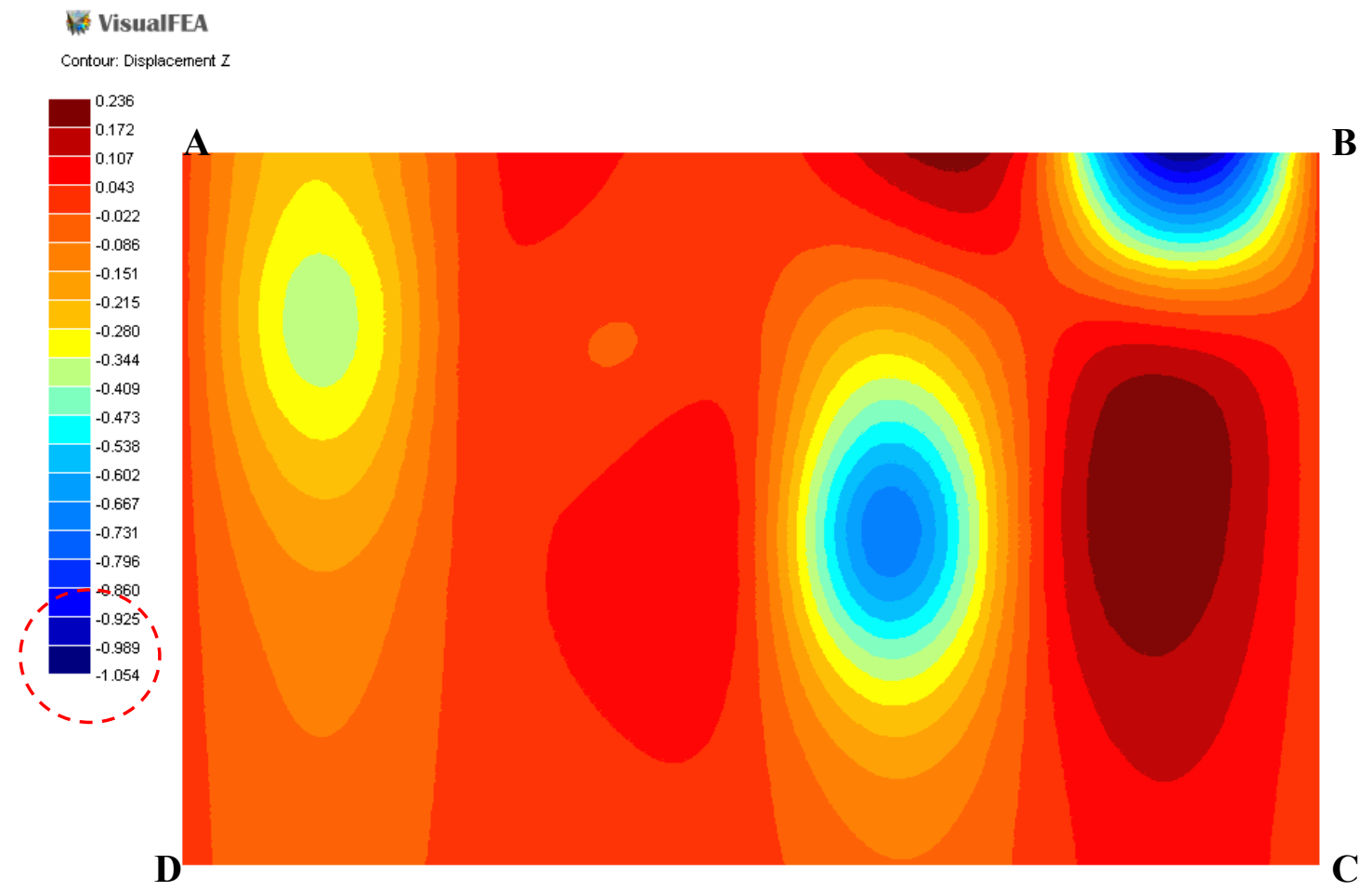

Figure 4-20. Module deformation [ft] in extreme condition 


\section{VisualFEA}

Contour: Displacement Z
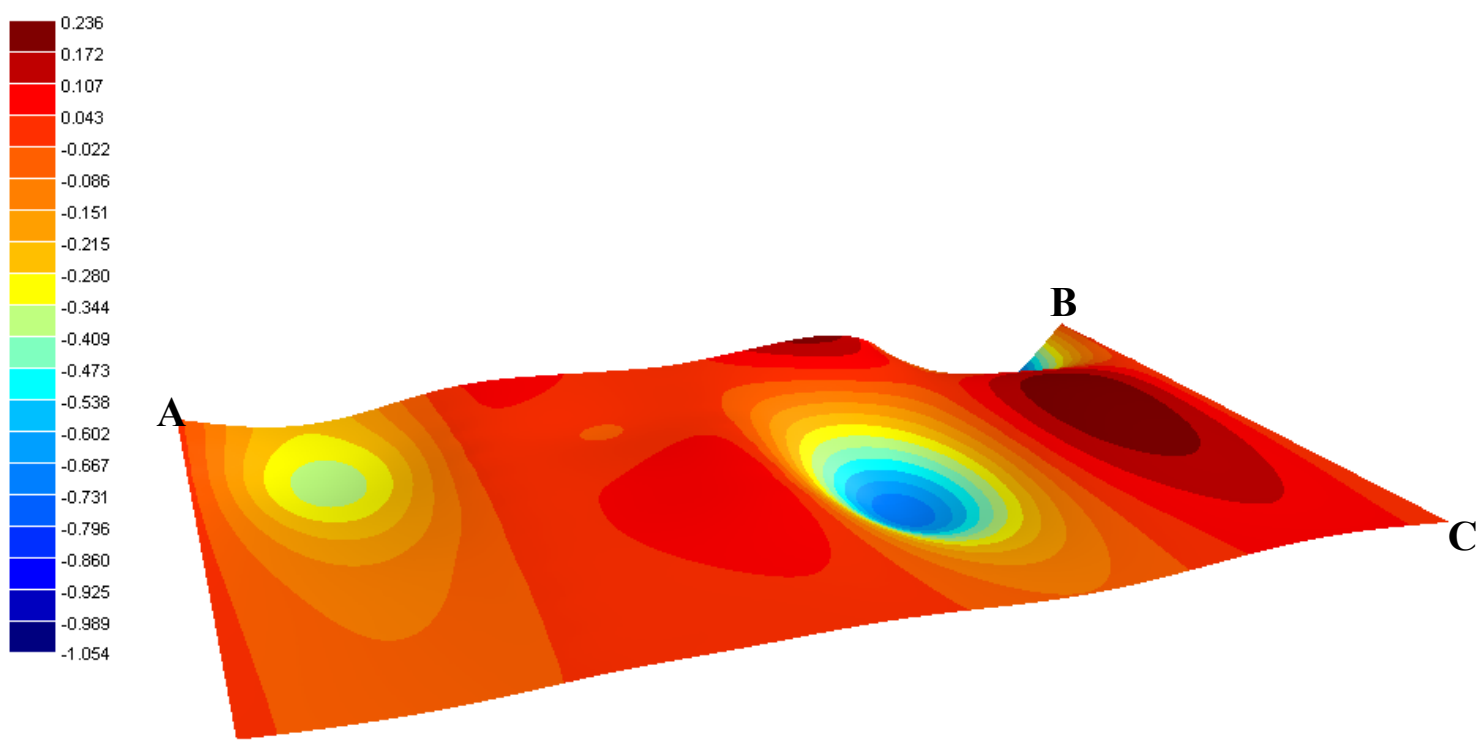

D

Figure 4-21. Module deformed shape in extreme condition

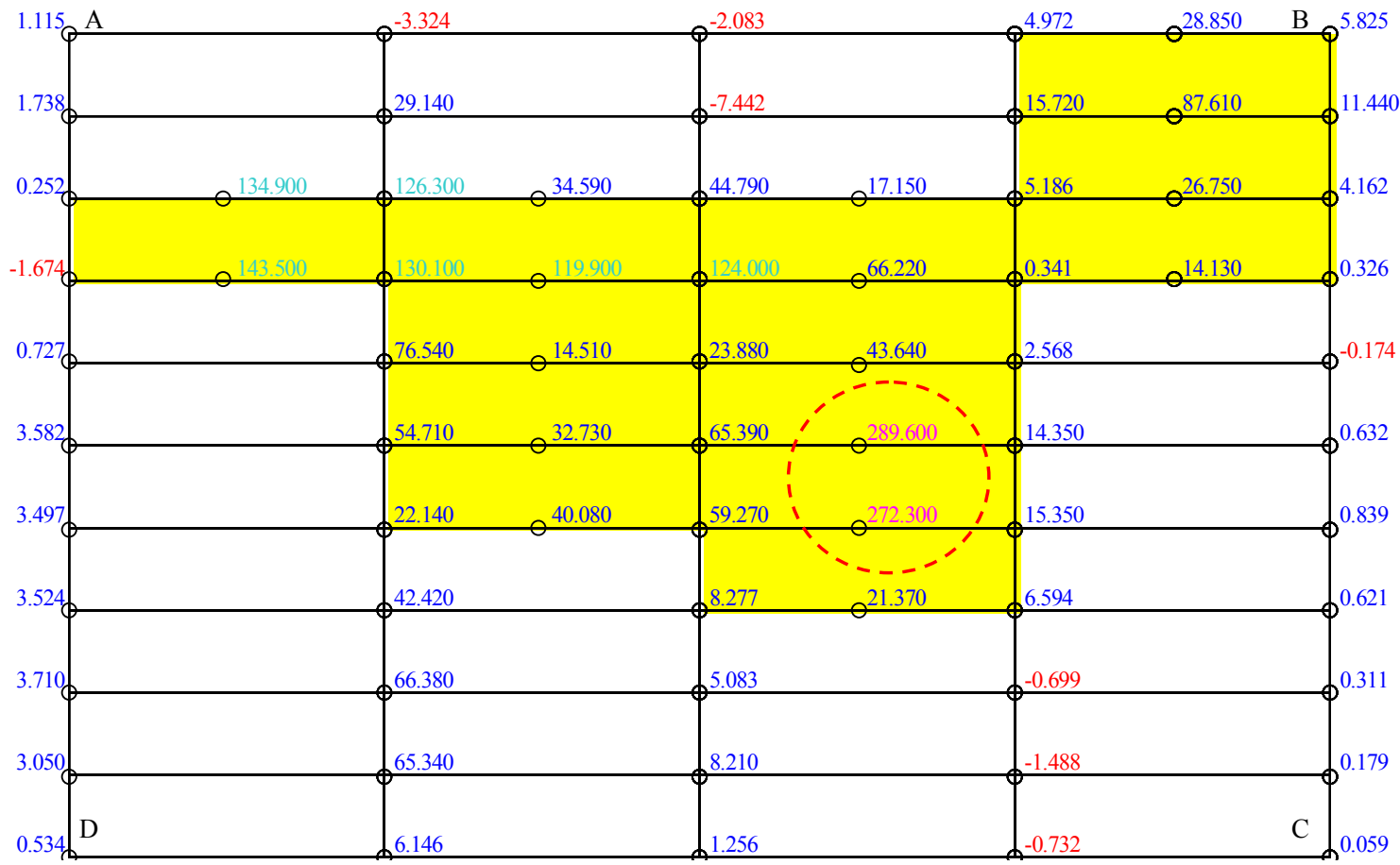

Figure 4-22. Pile reaction force [kips] in operating condition (using six piles) 


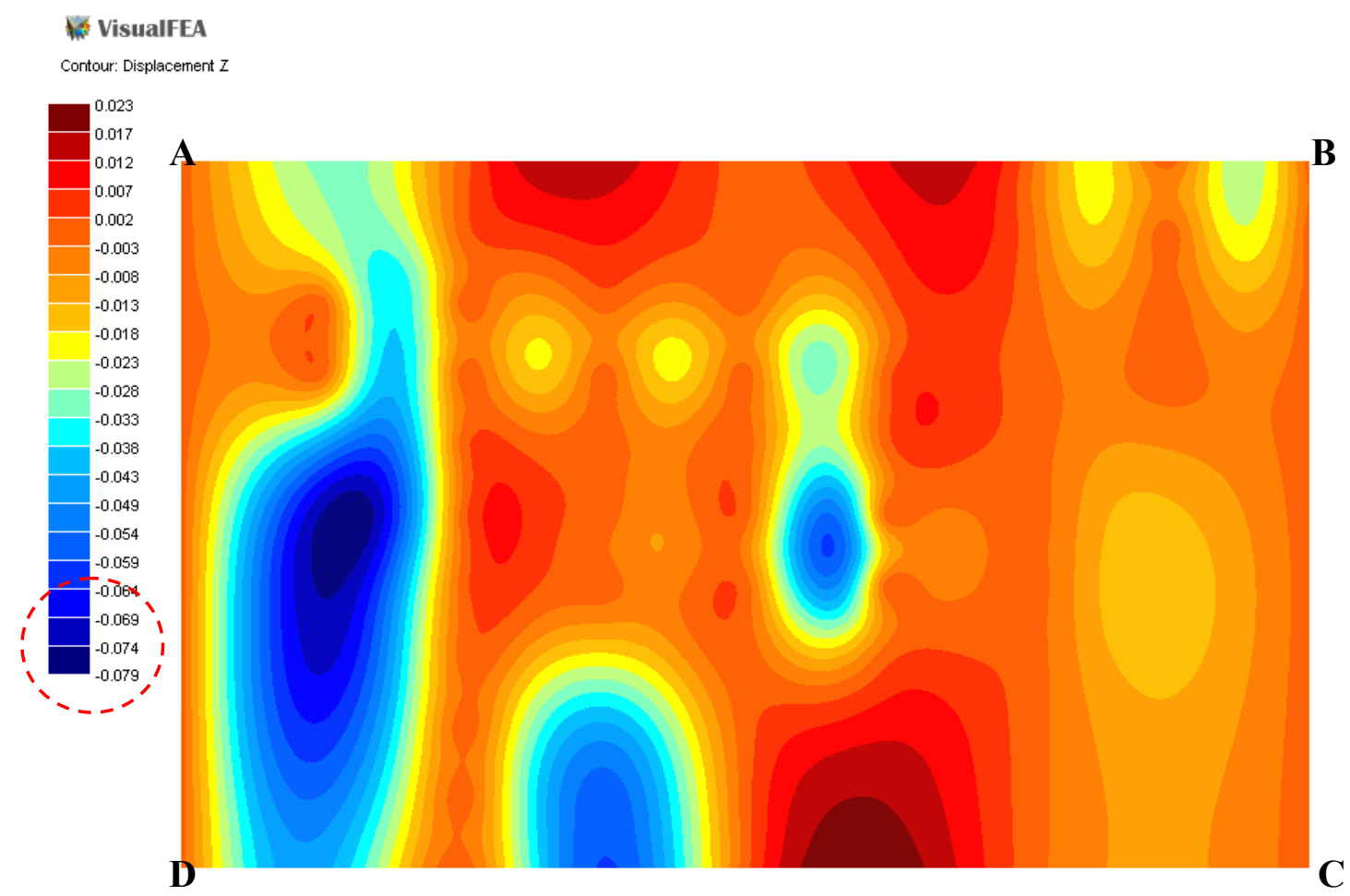

Figure 4-23. Module deformation [ft] in operating condition (using six piles)

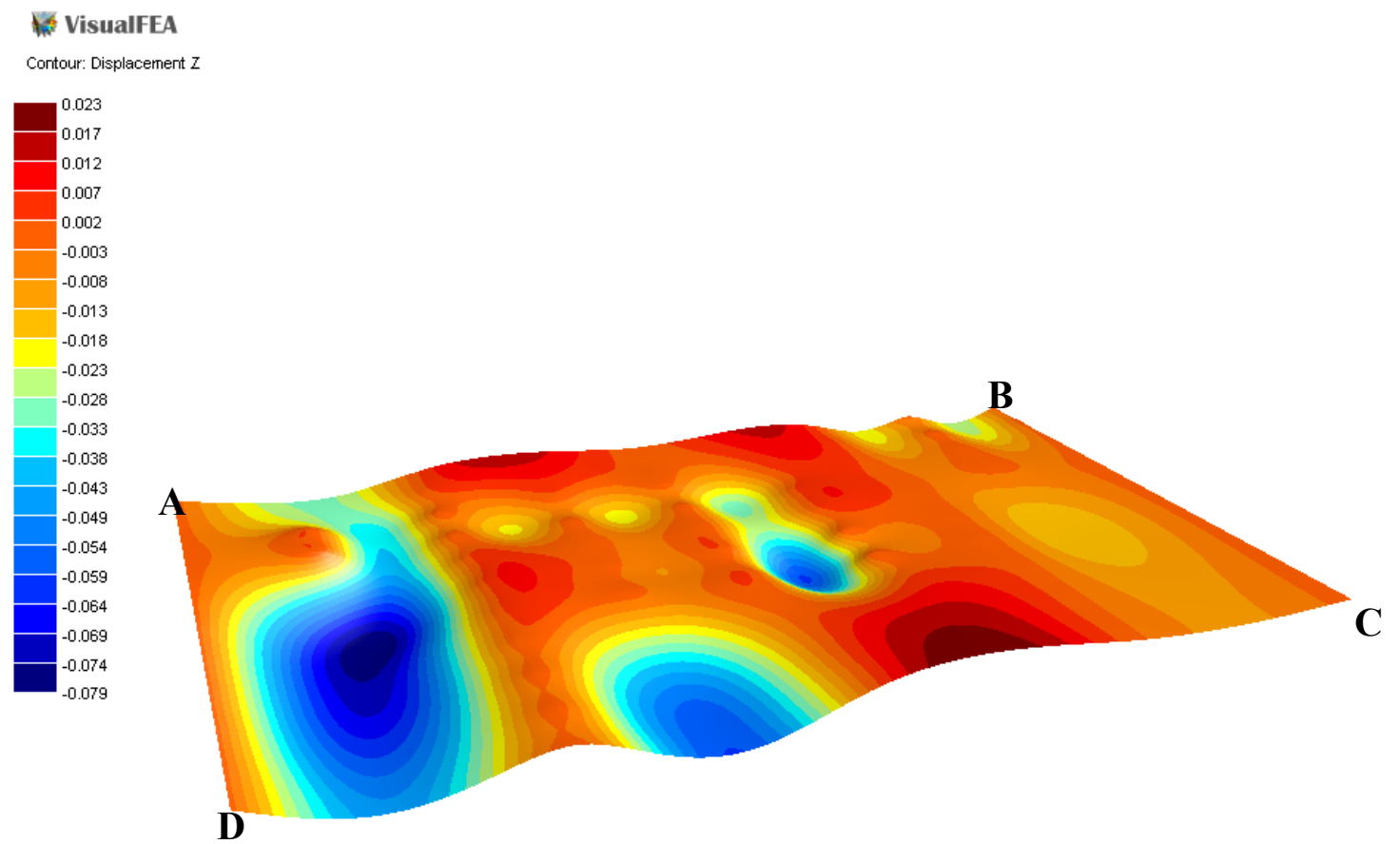

Figure 4-24. Module deformed shape in operating condition (using six piles) 


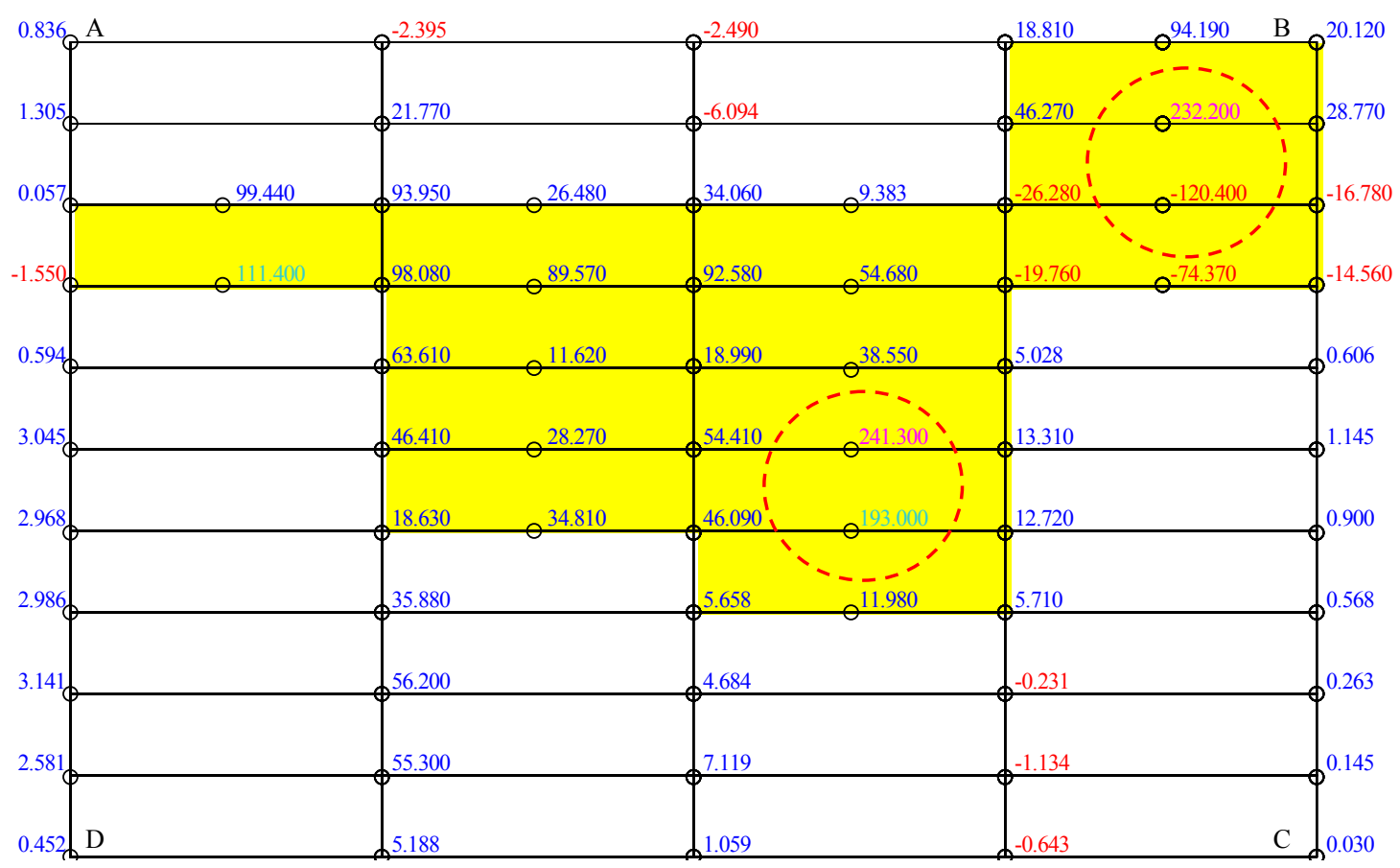

Figure 4-25. Pile reaction force [kips] in extreme condition (using six piles)

\section{VisualFEA}

Contour: Displacement Z

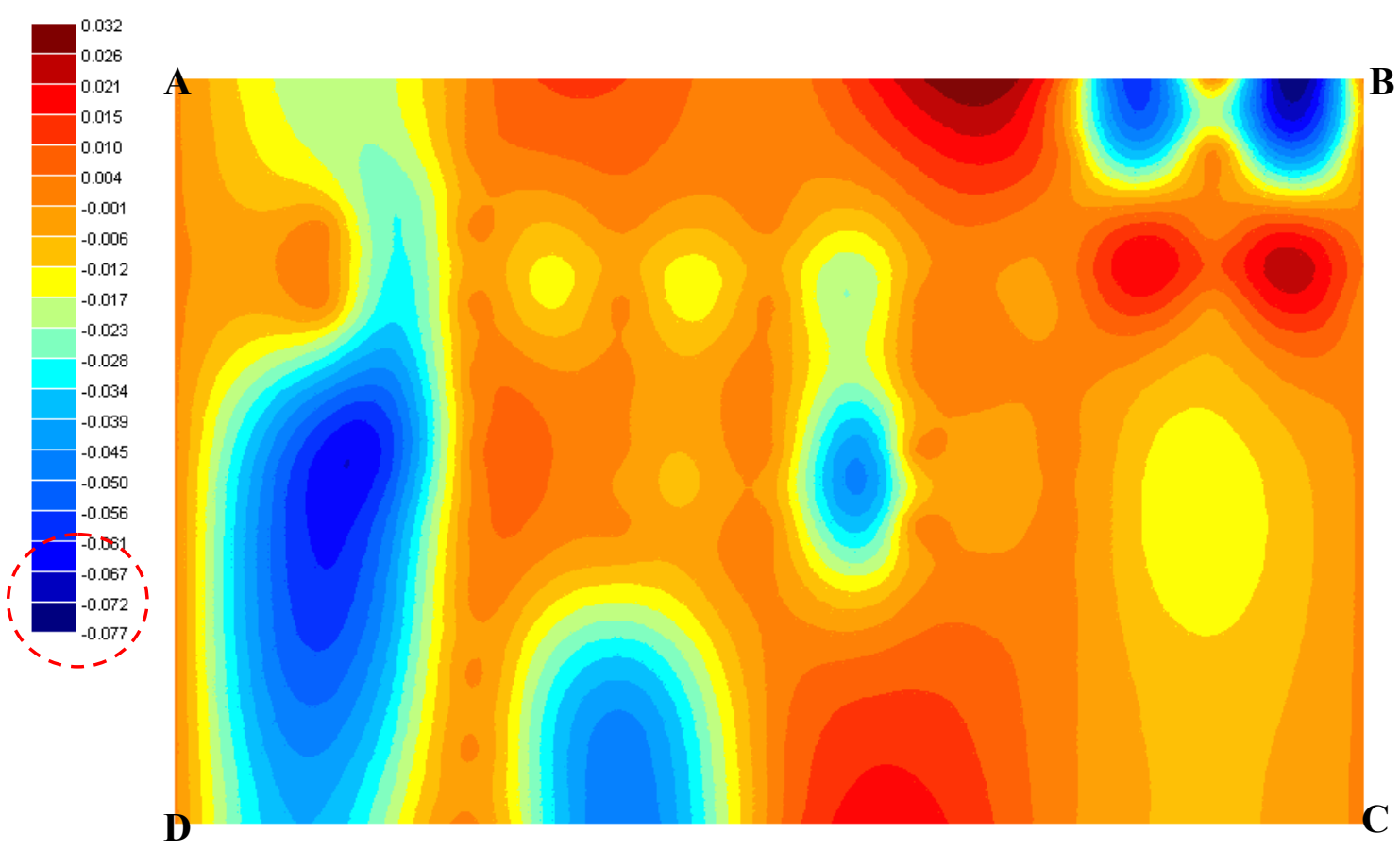

Figure 4-26. Module deformation [ft] in extreme condition (using six piles) 


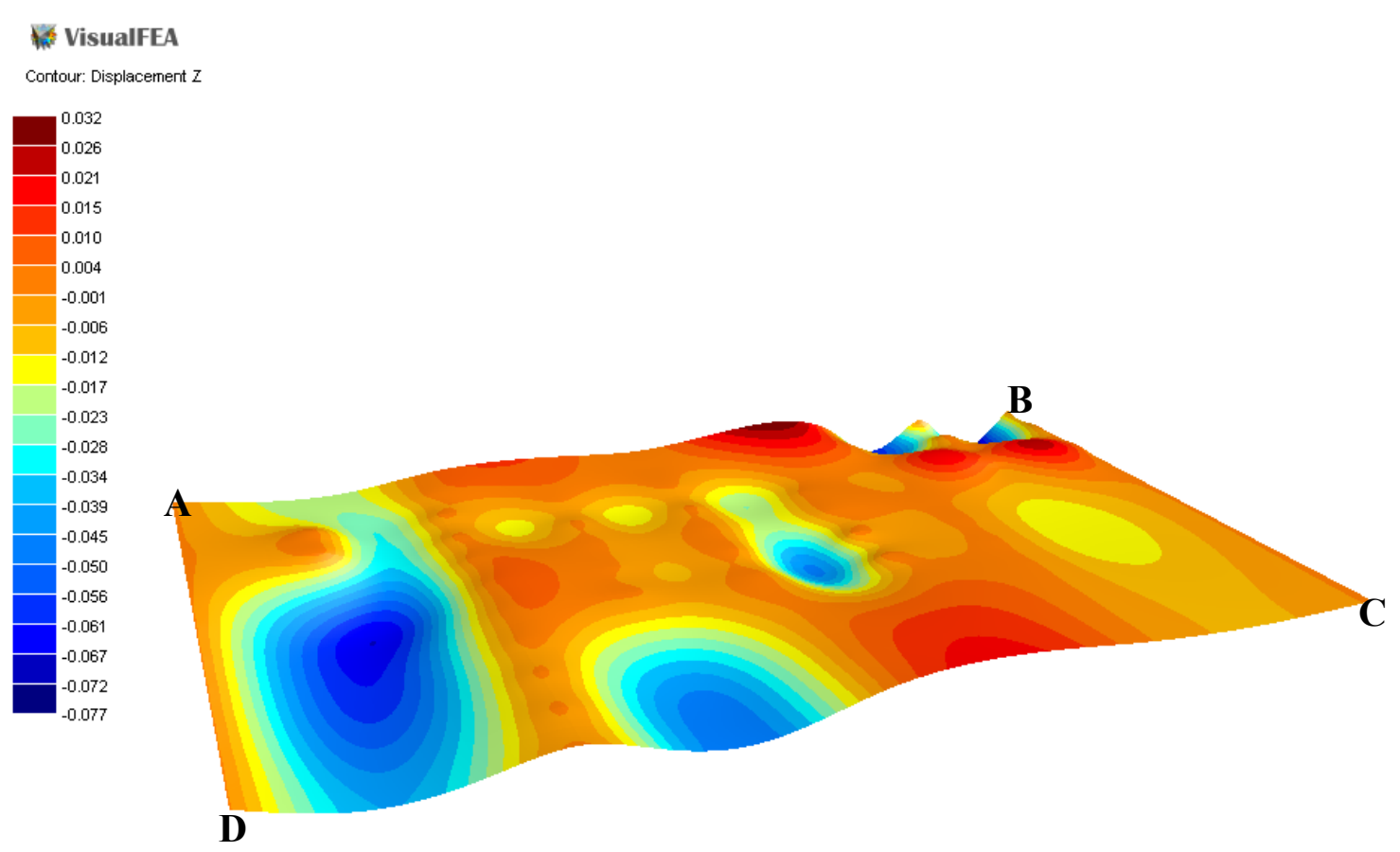

Figure 4-27. Module deformed shape in extreme condition (using six piles)

\subsubsection{LRFD Method}

In the case of Rapid Rig and the wind turbine, the pile capacity calculations are performed for the driven steel pipe piles and bored piles in accordance with the LRFD method. The maximum reaction force obtained from the 3-D simulations as described in Section 4.2.3.2 is considered as an applied load for generating the recommended pile selection tables in the Rapid Rig with wind turbine case similar to the tables in the general case. Since this force is the maximum value over the platform, it is conservative to choose one size of pile based on the maximum force and to apply it for all other piles.

As can be seen in Figure 4-22 and Figure 4-25, the reaction forces on most piles besides the four piles right underneath the mast and the wind turbine are significantly lower than the maximum reaction value. Therefore, if a single size of pile is used for the whole area, it is not an economical design. Instead, Table 4-3 and 4-6 developed in the general case can be used to choose a proper size for those piles subjected to relatively low reaction forces. 


\subsubsection{Results Summary}

Based on the pile capacity calculations in the case of Rapid Rig with the wind turbine, two tables (Table 4-13 4-14) are developed to provide a simple way to choose an appropriate pile size for various soil conditions according to the LRFD method.

Table 4-13. Recommended size of driven piles in the Rapid Rig with wind turbine case

\begin{tabular}{|c|c|c|c|c|c|c|c|}
\hline \multirow{3}{*}{$\begin{array}{l}\text { Weight of } \\
\text { Rigs \& } \\
\text { Accessories } \\
\text { (Unfactored) }\end{array}$} & \multirow[b]{3}{*}{$\begin{array}{l}\text { Factored } \\
\text { max. vertical } \\
\text { loads on one pile }\end{array}$} & \multicolumn{3}{|c|}{ Sand \& Gravels } & \multicolumn{3}{|c|}{ Silts \& Clays } \\
\hline & & very loose & medium & very dense & soft & medium & hard \\
\hline & & $\begin{array}{c}\gamma_{\mathrm{sat}}=115 \mathrm{pcf} \\
\text { G.W.L }=0 \mathrm{ft} \\
\mathrm{NSPT}=10 \mathrm{bpf}\end{array}$ & $\begin{array}{l}\gamma_{\mathrm{sat}}=120 \mathrm{pcf} \\
\text { G.W.L }=10 \mathrm{ft} \\
\text { NSPT }=30 \mathrm{bpf}\end{array}$ & $\begin{array}{l}\gamma_{\text {sat }}=127 \mathrm{pcf} \\
\text { G.W.L }=20 \mathrm{ft} \\
\text { NsPT }=50 \mathrm{bpf}\end{array}$ & $\begin{array}{l}\gamma_{\mathrm{sat}}=115 \mathrm{pcf} \\
\mathrm{G} . \mathrm{W} . \mathrm{L}=0 \mathrm{ft} \\
\mathrm{Su}=0.25 \mathrm{Po}^{\prime}\end{array}$ & $\begin{array}{c}\gamma_{\mathrm{sat}}=120 \mathrm{pcf} \\
\text { G.W.L }=10 \mathrm{ft} \\
\mathrm{Su}=60 \mathrm{kPa}\end{array}$ & $\begin{array}{l}\gamma_{\mathrm{sat}}=127 \mathrm{pcf} \\
\text { G.W.L }=20 \mathrm{ft} \\
\mathrm{Su}=100 \mathrm{kPa}\end{array}$ \\
\hline \multirow{2}{*}{1748 kips } & \multirow{2}{*}{289.6 kips } & $\mathrm{D}=24 \mathrm{in}$. & $\mathrm{D}=24 \mathrm{in}$ & $\mathrm{D}=16 \mathrm{in}$. & $\mathrm{D}=24 \mathrm{in}$. & $\mathrm{D}=24 \mathrm{in}$. & $\mathrm{D}=24 \mathrm{in}$ \\
\hline & & $\mathrm{L}=84 \mathrm{ft}$ & $\mathrm{L}=43 \mathrm{ft}$ & $\mathrm{L}=33 \mathrm{ft}$ & $\mathrm{L}=96 \mathrm{ft}$ & $\mathrm{L}=68 \mathrm{ft}$ & $\mathrm{L}=49 \mathrm{ft}$ \\
\hline
\end{tabular}

(where, $\mathrm{P}_{\mathrm{o}}{ }^{\prime}=$ effective overburden pressure, G.W.L. = ground water depth measured from the ground surface,

Table 4-14. Recommended size of bored piles in the Rapid Rig with wind turbine case

\begin{tabular}{|c|c|c|c|c|c|c|c|}
\hline \multirow{3}{*}{$\begin{array}{l}\text { Weight of } \\
\text { Rigs \& } \\
\text { Accessories } \\
\text { (Unfactored) }\end{array}$} & \multirow{3}{*}{$\begin{array}{l}\text { Soil types } \\
\text { ored } \\
\text { vertical } \\
\text { on one pile }\end{array}$} & \multicolumn{3}{|c|}{ Sand \& Gravels } & \multicolumn{3}{|c|}{ Silts \& Clays } \\
\hline & & very loose & medium & very dense & soft & medium & hard \\
\hline & & $\begin{array}{c}\gamma_{\mathrm{sat}}=115 \mathrm{pcf} \\
\text { G.W.L }=0 \mathrm{ft} \\
\text { NSPT }=10 \mathrm{bpf}\end{array}$ & $\begin{array}{l}\gamma_{\mathrm{sat}}=120 \mathrm{pcf} \\
\text { G.W.L }=10 \mathrm{ft} \\
\text { NSPT }=30 \mathrm{bpf}\end{array}$ & $\begin{array}{l}\gamma_{\mathrm{sat}}=127 \mathrm{pcf} \\
\mathrm{G} . \mathrm{W} \cdot \mathrm{L}=20 \mathrm{ft} \\
\mathrm{NSPT}=50 \mathrm{bpf}\end{array}$ & $\begin{array}{l}\gamma_{\mathrm{sat}}=115 \mathrm{pcf} \\
\mathrm{G} . \mathrm{W} . \mathrm{L}=0 \mathrm{ft} \\
\mathrm{Su}=0.25 \mathrm{P}_{\mathrm{o}}{ }^{\prime}\end{array}$ & $\begin{array}{c}\gamma_{\mathrm{sat}}=120 \mathrm{pcf} \\
\mathrm{G} . \mathrm{W} \cdot \mathrm{L}=10 \mathrm{ft} \\
\mathrm{Su}=60 \mathrm{kPa}\end{array}$ & $\begin{array}{l}\gamma_{\mathrm{sat}}=127 \mathrm{pcf} \\
\mathrm{G} \cdot \mathrm{W} \cdot \mathrm{L}=20 \mathrm{ft} \\
\mathrm{Su}=100 \mathrm{kPa}\end{array}$ \\
\hline \multirow{2}{*}{1748 kips } & \multirow{2}{*}{289.6 kips } & $\mathrm{D}=24 \mathrm{in}$. & $\mathrm{D}=24 \mathrm{in}$. & $\mathrm{D}=24 \mathrm{in}$ & $\mathrm{D}=24 \mathrm{in}$. & $\mathrm{D}=24 \mathrm{in}$. & $\mathrm{D}=24 \mathrm{in}$ \\
\hline & & $\mathrm{L}=76 \mathrm{ft}$ & $\mathrm{L}=40 \mathrm{ft}$ & $\mathrm{L}=29 \mathrm{ft}$ & $\mathrm{L}=137 \mathrm{ft}$ & $\mathrm{L}=104 \mathrm{ft}$ & $\mathrm{L}=62 \mathrm{ft}$ \\
\hline
\end{tabular}

(where, $\mathrm{P}_{\mathrm{o}}{ }^{\prime}=$ effective overburden pressure, G.W.L. = ground water depth measured from the ground surface,

\subsubsection{Construction Strategies of Pile Foundation}

In this section, four different construction methods of a driven steel pipe pile for an elevated platform with Rapid Rig are described for one specific soil condition with the LRFD method. The soil condition is assumed "Very dense sand" and the required soil parameters are shown in Figure 4-28.

Rapid Rig is placed on the platform as shown in Figure 4-29. It is noted that the layout shown in Figure 4-29 is not the same as the one shown in Figure 4-4. This is because EFD subject matter experts decided to reduce the number of modules being used in this study as many as possible since the cost of each module is very high. The refined 
platform consists of 24 aluminum modules (40 modules were initially used in Figure 44) and 45 piles as shown in Figure 4-30. Figure 4-30 also shows the reaction force on each pile and each pile is numbered as shown in Figure 4-31. The dimension of each component of Rapid Rig is provided by National Oilwell Varco.

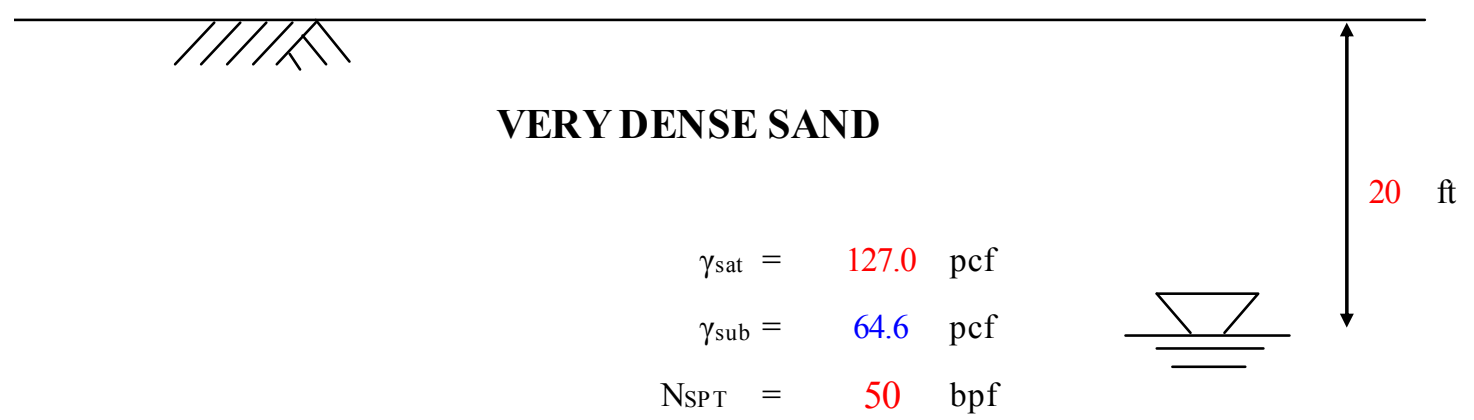

Figure 4-28. Assumed soil condition

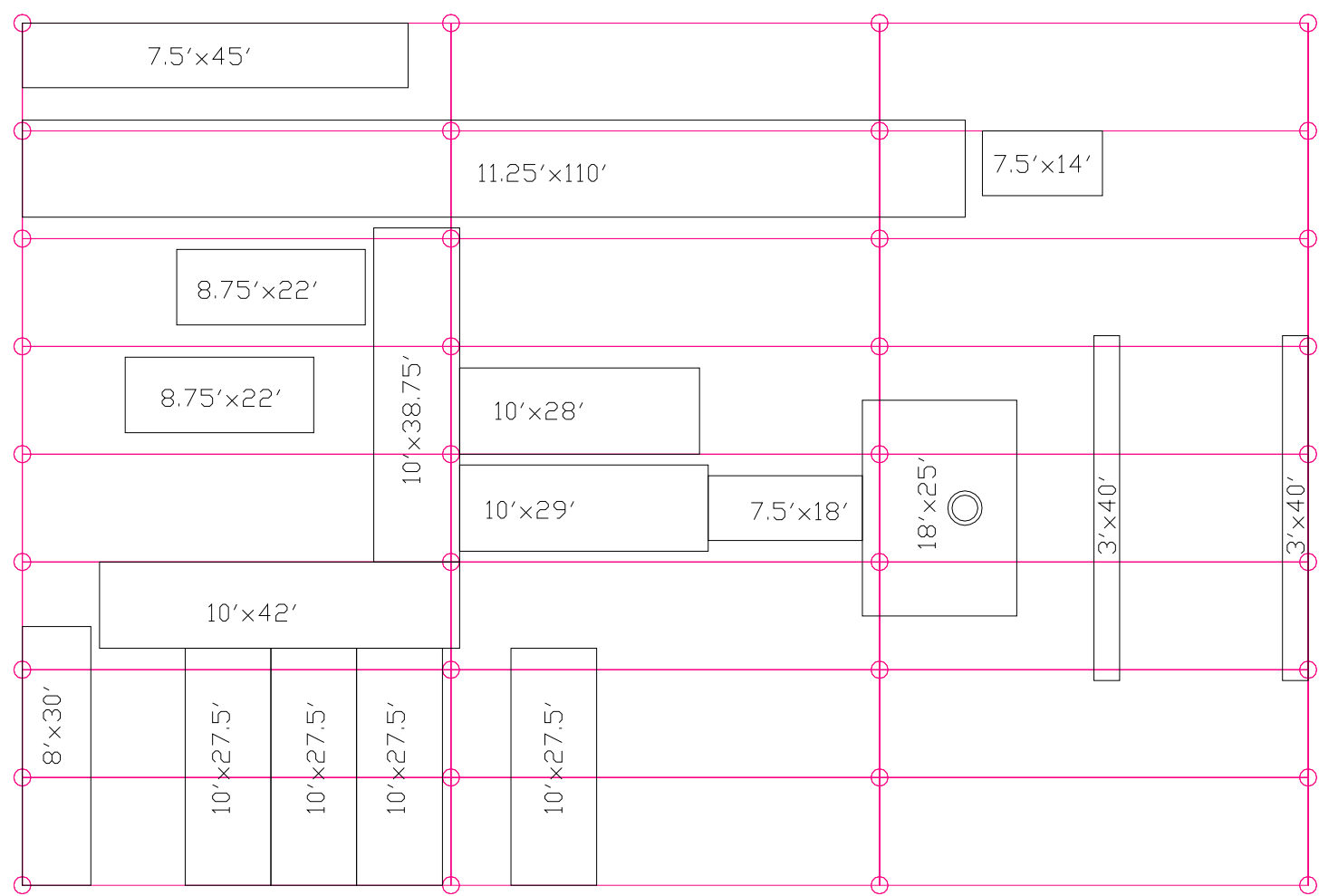

Figure 4-29. Layout of Rapid Rig on the elevated platform 


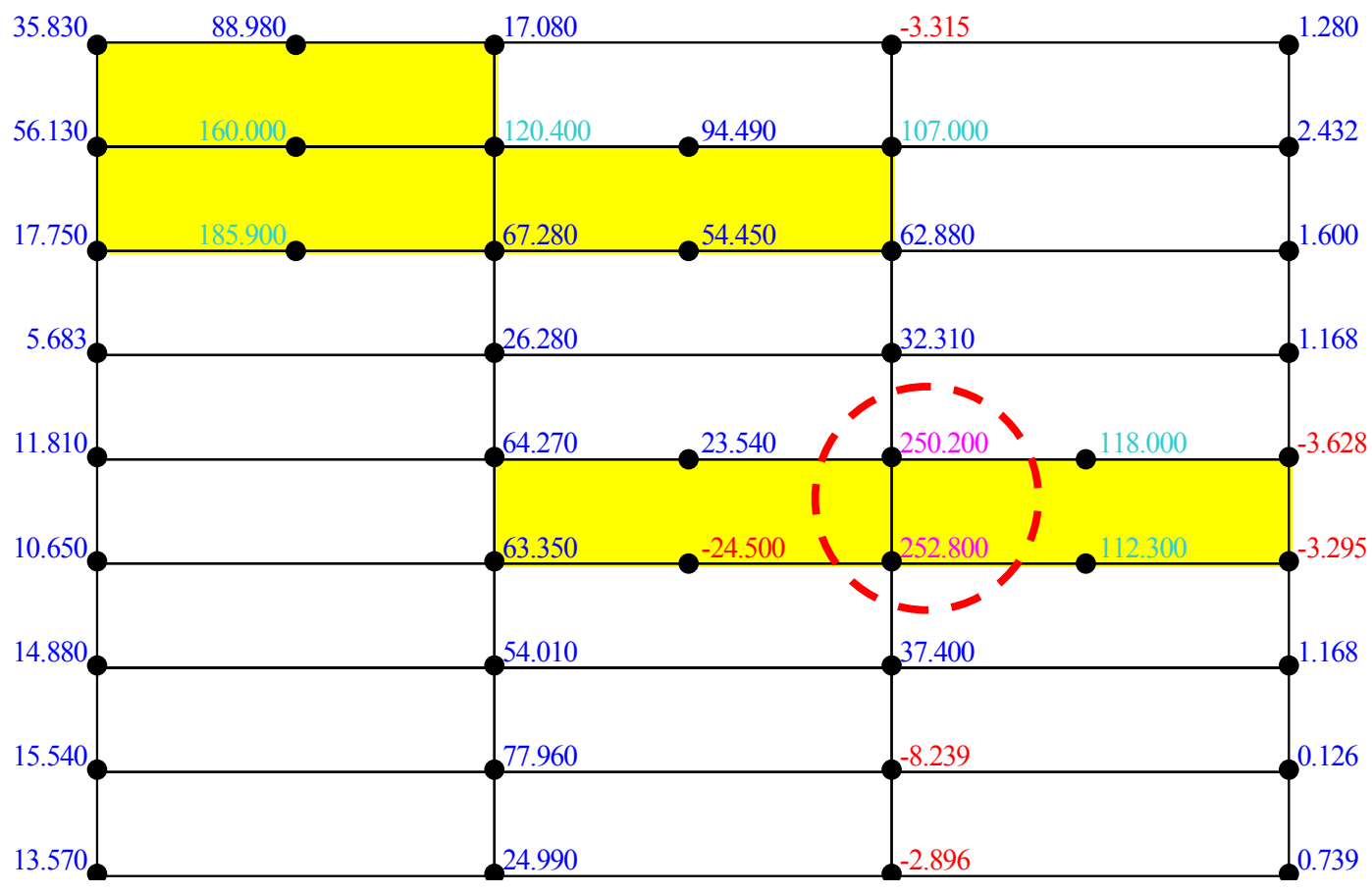

Figure 4-30. Reaction force on each pile [kips]

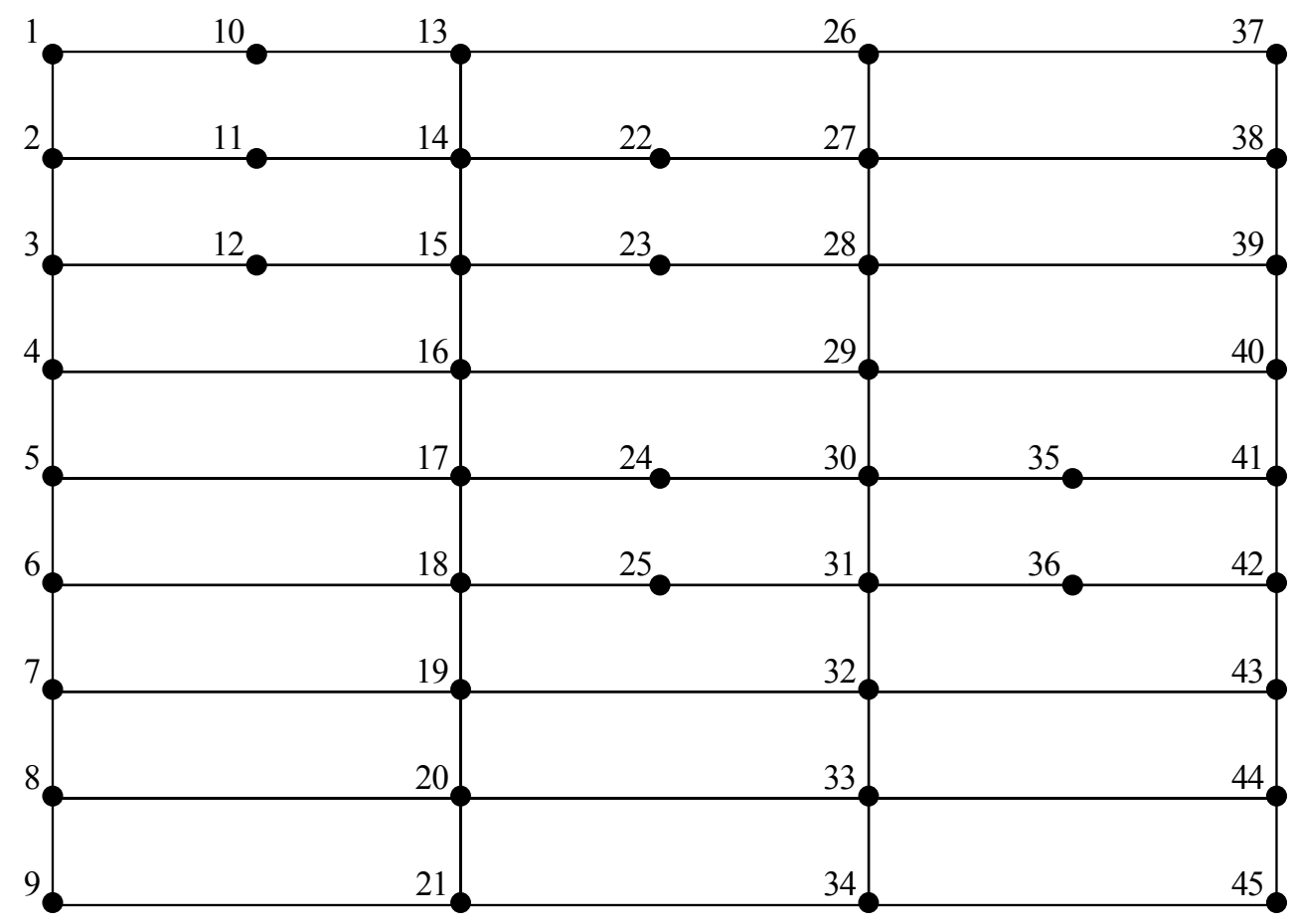

Figure 4-31. Numbers assigned to each pile 
In order to estimate the cost of pile foundations, 'RSMeans (2006)', the reference book of construction cost information, and several EFD experts' inputs are used in this study. After gathering the cost information, Eq. (4-3) is developed to be used for the cost estimation of piles. Eq. (4-3) is the best fit for the piles, the range from 10 to 24 inches in diameter and from 10 to 100 feets in length.

$$
\operatorname{Cost}=[50+(\mathrm{D}-10) \times 2.8] \times \mathrm{L}
$$

where $\mathrm{D}$ is the diameter in inches and $\mathrm{L}$ is the length in feets.

Four different construction strategies of pile foundation are described as follows:

1. Optimal pile size for each pile: for each pile with its reaction force, an exhaustive search optimization routine is run to find the optimized pile size (i.e., diameter and length). Once all possible pile sizes, which satisfy the pile capacity design criteria described in Section 3.2, have been evaluated, the size with the lowest cost is the best pile size. Table 4-15 shows the optimized pile size and cost for each pile.

2. Using same piles for the entire platform area: This is the simplest method to construct piles. Since no. 31 and no. 32 piles shown in Figure 4-31 sustain the biggest applied load among all 45 piles, the pile size of no. 31 and no. 32 are used for the entire platform area. Table 4-16 shows the total cost of using this method.

3. Using two piles: Since the reaction forces on only two piles (no. 31 and 32) are significantly greater than other piles, two pile sizes are used. One is for those two piles (no. 31 and 32) and the other one is for the remaining piles. Table 4-17 shows the total cost of using this method.

4. Categorized by reaction forces: This method categorizes pile size by three different reaction forces: (1) the reaction force is greater than $200 \mathrm{kips}$; (2) the reaction force is greater than $100 \mathrm{kips}$ and less than or equal to $200 \mathrm{kips}$; (3) the reaction force is less than or equal to 100 kips. Table 4-18 shows the total cost of using this method. 
Table 4-15. Optimal pile size and cost for each pile

\begin{tabular}{|c|c|c|c|c|c|c|c|c|c|}
\hline $\begin{array}{c}\text { Pile } \\
\text { no. }\end{array}$ & $\begin{array}{c}\text { Diameter } \\
\text { (in.) }\end{array}$ & $\begin{array}{c}\text { Length } \\
\text { (ft) }\end{array}$ & Quantities & Total & $\begin{array}{c}\text { Pile } \\
\text { no. }\end{array}$ & $\begin{array}{c}\text { Diameter } \\
\text { (in.) }\end{array}$ & $\begin{array}{c}\text { Length } \\
(\mathrm{ft})\end{array}$ & Quantities & Total \\
\hline 1 & 10 & 11 & 1 & $\$ 550.00$ & 24 & 10 & 10 & 1 & $\$ 500.00$ \\
\hline 2 & 14 & 10 & 1 & $\$ 612.00$ & 25 & 12 & 16 & 1 & $\$ 889.60$ \\
\hline 3 & 10 & 10 & 1 & $\$ 500.00$ & 26 & 10 & 10 & 1 & $\$ 500.00$ \\
\hline 4 & 10 & 10 & 1 & $\$ 500.00$ & 27 & 20 & 10 & 1 & $\$ 780.00$ \\
\hline 5 & 10 & 10 & 1 & $\$ 500.00$ & 28 & 16 & 10 & 1 & $\$ 668.00$ \\
\hline 6 & 10 & 10 & 1 & $\$ 500.00$ & 29 & 10 & 10 & 1 & $\$ 500.00$ \\
\hline 7 & 10 & 10 & 1 & $\$ 500.00$ & 30 & 24 & 15 & 1 & $\$ 1,338.00$ \\
\hline 8 & 10 & 10 & 1 & $\$ 500.00$ & 31 & 24 & 15 & 1 & $\$ 1,338.00$ \\
\hline 9 & 10 & 10 & 1 & $\$ 500.00$ & 32 & 12 & 10 & 1 & $\$ 556.00$ \\
\hline 10 & 18 & 10 & 1 & $\$ 724.00$ & 33 & 10 & 11 & 1 & $\$ 550.00$ \\
\hline 11 & 24 & 11 & 1 & $\$ 981.20$ & 34 & 10 & 10 & 1 & $\$ 500.00$ \\
\hline 12 & 24 & 12 & 1 & $\$ 1,070.40$ & 35 & 22 & 10 & 1 & $\$ 836.00$ \\
\hline 13 & 10 & 10 & 1 & $\$ 500.00$ & 36 & 20 & 10 & 1 & $\$ 780.00$ \\
\hline 14 & 22 & 10 & 1 & $\$ 836.00$ & 37 & 10 & 10 & 1 & $\$ 500.00$ \\
\hline 15 & 16 & 10 & 1 & $\$ 668.00$ & 38 & 10 & 10 & 1 & $\$ 500.00$ \\
\hline 16 & 10 & 10 & 1 & $\$ 500.00$ & 39 & 10 & 10 & 1 & $\$ 500.00$ \\
\hline 17 & 16 & 10 & 1 & $\$ 668.00$ & 40 & 10 & 10 & 1 & $\$ 500.00$ \\
\hline 18 & 16 & 10 & 1 & $\$ 668.00$ & 41 & 10 & 10 & 1 & $\$ 500.00$ \\
\hline 19 & 14 & 10 & 1 & $\$ 612.00$ & 42 & 10 & 10 & 1 & $\$ 500.00$ \\
\hline 20 & 18 & 10 & 1 & $\$ 724.00$ & 43 & 10 & 10 & 1 & $\$ 500.00$ \\
\hline 21 & 10 & 10 & 1 & $\$ 500.00$ & 44 & 10 & 10 & 1 & $\$ 500.00$ \\
\hline 22 & 20 & 10 & 1 & $\$ 780.00$ & 45 & 10 & 10 & 1 & $\$ 500.00$ \\
\hline 23 & 14 & 10 & 1 & $\$ 612.00$ & & & $\Sigma$ & $\underline{45}$ & $\$ 28,741.20$ \\
\hline
\end{tabular}

Table 4-16. Pile size for "using same pile" method

\begin{tabular}{|c|c|c|c|c|}
\hline Pile no. & Diameter (in.) & Length (ft) & Quantities & Total \\
\hline all & 24 & 15 & 45 & $\$ 60,210.00$ \\
\hline & & $\Sigma$ & $\underline{45}$ & $\$ 60,210.00$ \\
\hline
\end{tabular}


Table 4-17. Pile size for "using two piles" method

\begin{tabular}{|c|c|c|c|c|}
\hline Pile no. & Diameter (in.) & Length (ft) & Quantities & Total \\
\hline $30 \& 31$ & 24 & 15 & 2 & $\$ 2,676.00$ \\
\hline others & 24 & 12 & 43 & $\$ 46,027.00$ \\
\hline
\end{tabular}

Table 4-18. Pile size for "categorized by reaction forces" method

\begin{tabular}{|c|c|c|c|c|}
\hline Category & Diameter (in.) & Length (ft) & Quantities & Total \\
\hline $\mathrm{P}>200$ kips & 24 & 15 & 2 & $\$ 2,676.00$ \\
\hline $100<\mathrm{P} \leq 200$ & 24 & 12 & 6 & $\$ 6,422.00$ \\
\hline$P \leq 100$ & 20 & 10 & 37 & $\$ 28,860.00$ \\
\hline
\end{tabular}

\subsubsection{Lessons Learned}

Conventional onshore drilling for oil and gas consists of placing a gravel pad for leveling and carrying capacity purposes. The use of an elevated platform as an alternative to the gravel pad is less intrusive and leads to a more environmentally friendly approach to oil and gas drilling. Since elevated drilling platforms require the use of piles, many different cases of pile design are conducted through Section 4.2 to give site location engineers a basic idea about pile foundation designs of a platform for various platform weights and soil conditions. The four different construction strategies of pile foundation are also described in Section 4.2.4. "Using optimal pile size for each pile" method is the least expensive method while "using same pile size for the entire platform" method is the most expensive method. However, in real construction, some other construction factors such as pile set up time and possibility of wrong pile placement are also required to be considered. Therefore, site location engineers should select the appropriate pile construction strategy based on each site condition. 


\subsection{Feasibility of Using Composite Mat System in Drill Sites}

Another alternative of environmentally friendly foundations for drill sites is composite mats. Since the total construction cost of an elevated platform is considerably high and the construction is time consuming, a composite mat system can be a good alternative to the gravel pad. DURA-BASE Composite Mat System from Newpark mats and Integrated Services is considered for the feasibility study in this section.

\subsubsection{Specification of DURA-BASE Composite Mat System}

The large size of DURA-BASE Composite Mat System is used in this feasibility study. Table 4-19 shows the specification of this mat. More specific information about this mat system can be found in the following website (http://www.newparkmats.com).

Table 4-19. Brief information about DURA-BASE Composite Mat System

\begin{tabular}{|l|l|}
\hline Dimensions & $8 \mathrm{ft}$ wide, $14 \mathrm{ft}$ long, and 4 inches depth (for one layer) \\
\hline Weight & $1,050 \mathrm{lbs}$ \\
\hline Material & High density polyethylene \\
\hline Young's Modulus & $1 \mathrm{GPa} \approx 2.09 \mathrm{e}+07 \mathrm{psf}$ \\
\hline Purchase rate & $\$ 20.50 / \mathrm{ft}^{2}$ (the rate was obtained in 2006$)$ \\
\hline Rent rate $(90$ days) & $\$ 2.00 / \mathrm{ft}^{2}$ (the rate subject to change) \\
\hline
\end{tabular}

\subsubsection{Finite Element Analysis for the Composite Mat System}

In order to conduct a parametric study of the composite mat, a finite element mesh (i.e., two-dimensional axisymmetric mesh and three node triangular elements) is generated using a numerical analysis program, VisualFEA as shown in Figure 4-32. For this parametric study, the applied load area is varied from 6 inches to 10 feets in diameter (i.e., $\mathrm{D}=0.5,1.0,2.0,4.0,6.0$, and $10 \mathrm{ft}$ ) and the ratio of Young's Modulus between the composite mat and the soil is varied from 1 to 100 (i.e., 1, 10, 20, and 100). The results of the analysis are summarized by $\rho$-values. The $\rho$-values are calculated by $\mathrm{P}_{(\max )} / \mathrm{P}_{(\text {applied) }}$. The $\mathrm{P}_{\text {(applied) }}$ is the applied load on the mat system and the $\mathrm{P}_{(\max )}$ is the 
maximum pressure obtained from the ground. In this parametric analysis, the applied load is 1 psf and the result summary is shown in Table 4-20. In order to better display the results, result graphs are summarized as shown in Figure 4-33.

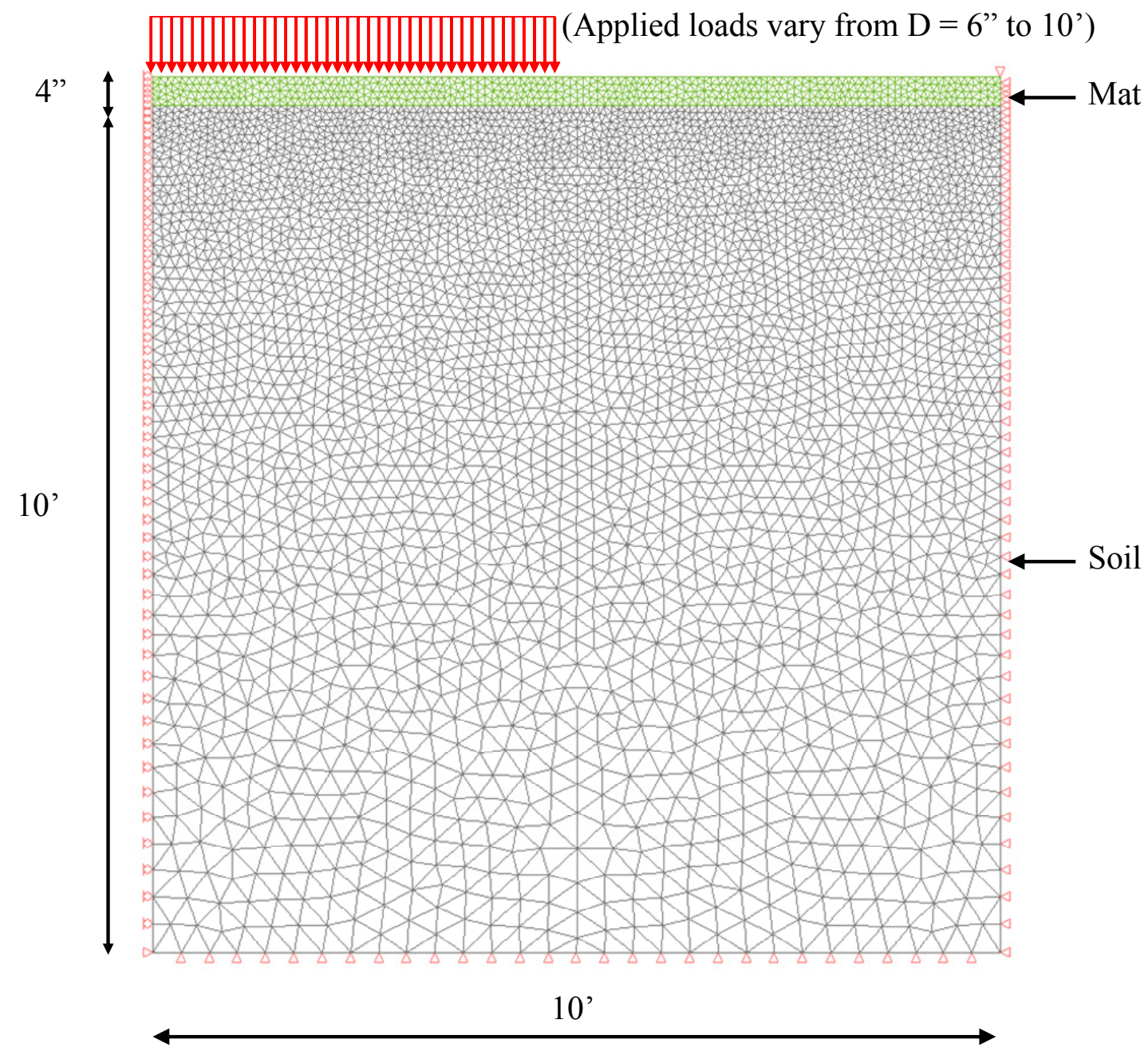

Figure 4-32. Actual mesh generated for this parametric analysis 
Table 4-20. $\left.\rho=\mathrm{P}_{(\max )} / \mathrm{P}_{(\text {applied }}\right)$ values

\begin{tabular}{|c|c|c|c|c|}
\hline \multicolumn{2}{|r|}{ Parameters } & No Mat & One Mat & Two Mats \\
\hline \multirow{4}{*}{$\mathrm{D}=0.5 \mathrm{ft}$} & $\mathrm{E}_{\text {(mat) }} / \mathrm{E}_{\text {(soil) }}=1$ & \multirow{4}{*}{1.000} & 0.437 & 0.175 \\
\hline & $\mathrm{E}_{\text {(mat) }} / \mathrm{E}_{\text {(soil) }}=10$ & & 0.218 & 0.077 \\
\hline & $\mathrm{E}_{\text {(mat) }} / \mathrm{E}_{\text {(soil) }}=20$ & & 0.158 & 0.053 \\
\hline & $\mathrm{E}_{\text {(mat) }} / \mathrm{E}_{\text {(soil) }}=100$ & & 0.064 & 0.020 \\
\hline \multirow{4}{*}{$\mathrm{D}=1 \mathrm{ft}$} & $\mathrm{E}_{(\mathrm{mat})} / \mathrm{E}_{(\text {soil) }}=1$ & \multirow{4}{*}{1.000} & 0.776 & 0.454 \\
\hline & $\mathrm{E}_{\text {(mat) }} / \mathrm{E}_{\text {(soil) }}=10$ & & 0.501 & 0.224 \\
\hline & $\mathrm{E}_{\text {(mat) }} / \mathrm{E}_{\text {(soil) }}=20$ & & 0.394 & 0.160 \\
\hline & $\mathrm{E}_{\text {(mat) }} / \mathrm{E}_{\text {(soil) }}=100$ & & 0.189 & 0.063 \\
\hline \multirow{4}{*}{$\mathrm{D}=2 \mathrm{ft}$} & $\mathrm{E}_{\text {(mat) }} / \mathrm{E}_{\text {(soil) }}=1$ & \multirow{4}{*}{1.000} & 0.955 & 0.807 \\
\hline & $\mathrm{E}_{\text {(mat) }} / \mathrm{E}_{\text {(soil) }}=10$ & & 0.853 & 0.519 \\
\hline & $\mathrm{E}_{\text {(mat) }} / \mathrm{E}_{\text {(soil) }}=20$ & & 0.771 & 0.409 \\
\hline & $\mathrm{E}_{(\mathrm{mat})} / \mathrm{E}_{(\text {soil })}=100$ & & 0.486 & 0.193 \\
\hline \multirow{4}{*}{$\mathrm{D}=4 \mathrm{ft}$} & $\mathrm{E}_{\text {(mat) }} / \mathrm{E}_{\text {(soil) }}=1$ & \multirow{4}{*}{1.000} & 0.991 & 0.961 \\
\hline & $\mathrm{E}_{\text {(mat) }} / \mathrm{E}_{\text {(soil) }}=10$ & & 0.992 & 0.856 \\
\hline & $\mathrm{E}_{\text {(mat) }} / \mathrm{E}_{(\text {(soil) }}=20$ & & 0.988 & 0.773 \\
\hline & $\mathrm{E}_{\text {(mat) }} / \mathrm{E}_{\text {(soil) }}=100$ & & 0.895 & 0.487 \\
\hline \multirow{4}{*}{$\mathrm{D}=6 \mathrm{ft}$} & $\mathrm{E}_{\text {(mat) }} / \mathrm{E}_{\text {(soil) }}=1$ & \multirow{4}{*}{1.000} & 0.995 & 0.985 \\
\hline & $\mathrm{E}_{\text {(mat) }} / \mathrm{E}_{\text {(soil) }}=10$ & & 0.993 & 0.962 \\
\hline & $\mathrm{E}_{\text {(mat) }} / \mathrm{E}_{\text {(soil) }}=20$ & & 1.000 & 0.936 \\
\hline & $\mathrm{E}_{\text {(mat) }} / \mathrm{E}_{\text {(soil) }}=100$ & & 1.000 & 0.729 \\
\hline \multirow{4}{*}{$\mathrm{D}=10 \mathrm{ft}$} & $\mathrm{E}_{\text {(mat) }} / \mathrm{E}_{(\text {soil })}=1$ & \multirow{4}{*}{1.000} & 0.999 & 0.996 \\
\hline & $\mathrm{E}_{\text {(mat) }} / \mathrm{E}_{\text {(soil) }}=10$ & & 0.994 & 0.987 \\
\hline & $\mathrm{E}_{\text {(mat) }} / \mathrm{E}_{\text {(soil) }}=20$ & & 0.996 & 0.984 \\
\hline & $\mathrm{E}_{\text {(mat) }} / \mathrm{E}_{\text {(soil) }}=100$ & & 1.000 & 0.972 \\
\hline
\end{tabular}




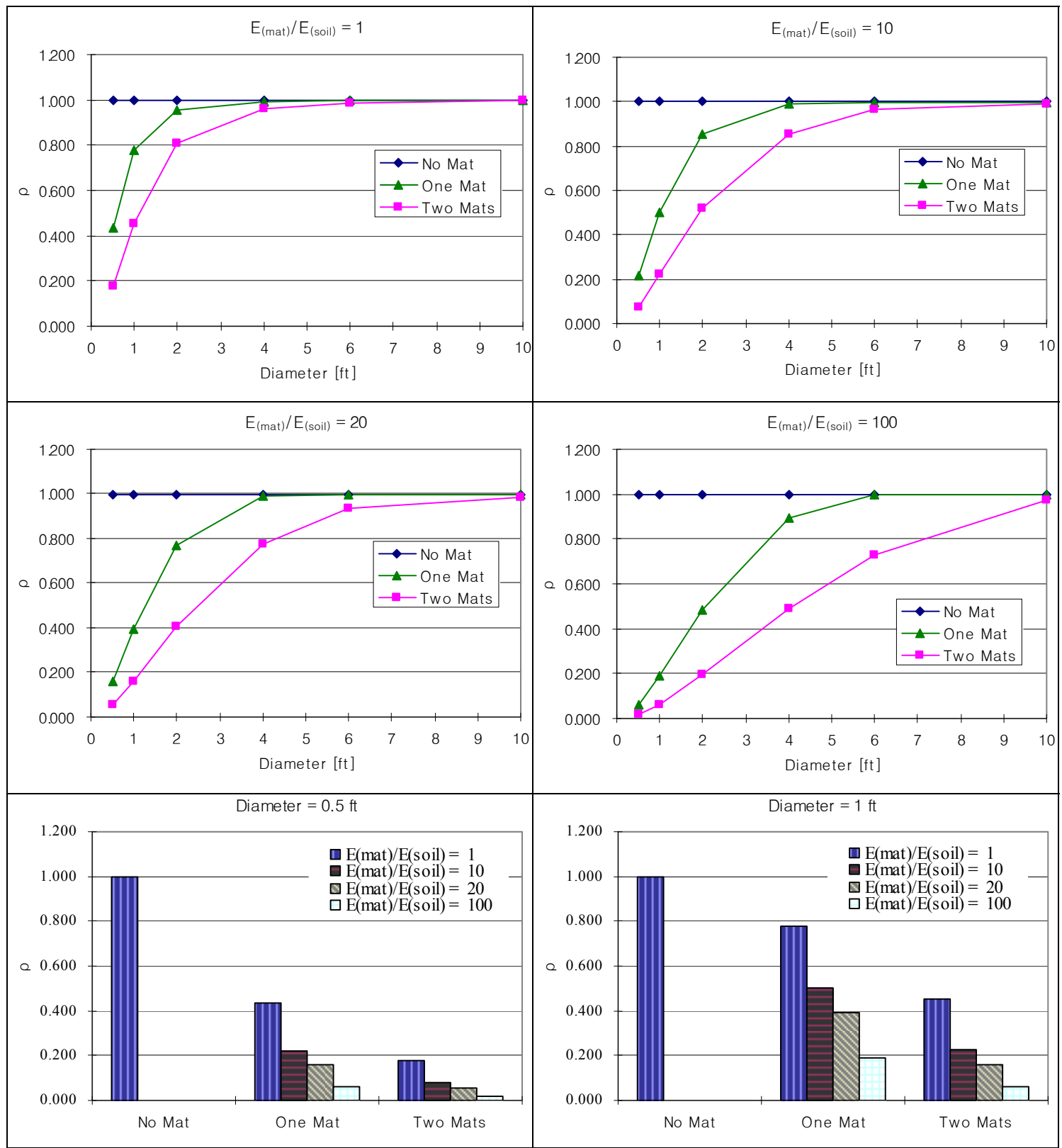

Figure 4-33. The result summary graphs 


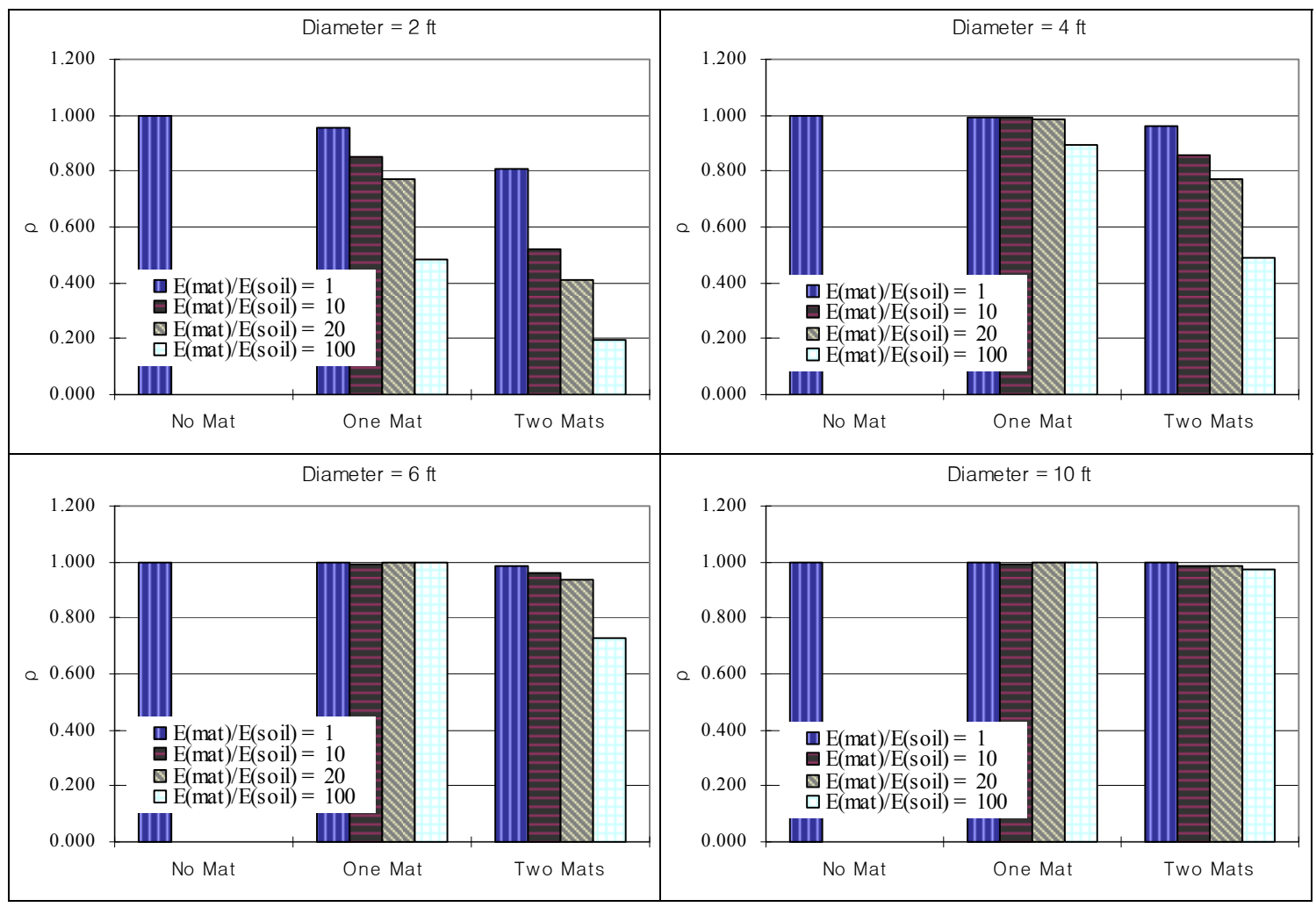

Figure 4-33. Continued

\subsubsection{Lessons Learned}

Throughout this parametric study, it is indicated that the single layer mat system (one mat) can decrease the pressure up to $95 \%$ for small loading areas (i.e., $\mathrm{D}=6$ " such as car or truck tire areas) over soft soil (i.e., Young's Modulus is less than $10 \mathrm{MPa}$ ). Therefore, the mat system seems to be very beneficial for traffic areas such as small tires over soft soils. On the other hand, the single layer mat system does not provide significant decrease in pressure for large loading areas (i.e., D $>$ 6') over stiff soil (i.e., Young's Modulus is greater than $50 \mathrm{MPa}$ ). Therefore, the mat system seems not to be significantly beneficial for large bins on desert soils. The double layer mat system (two mats) also looks beneficial for small loading areas but as applied load area increases, it seems to lose the benefit of using it especially on hard soils. 


\section{SYSTEMS APPROACH FOR TECHNOLOGY SELECTION}

\subsection{Research Methodology}

The main purpose of this research is to help decision-makers select an optimal drilling system for a given site in order to minimize environmental impact and maximize profit at that specific site. A technology evaluation protocol has been developed by EFD project participants and then refined based on EFD experts' inputs and feedbacks. Further interaction with appropriate experts would be valuable in revising this evaluation protocol. The overall procedure is briefly illustrated as follows:

Step 1: Identify main subsystems, subsets, and technologies within each subset for the EFD operations.

Step 2: Define attributes and develop attribute scales to evaluate technologies.

Step 3: Assign scores to all technologies using the attribute scales.

Step 4: For each attribute, calculate the overall attribute score of a system by adding the technology scores or selecting the minimum technology score.

Step 5: For each attribute and in order to homogenize the scores, develop a "utility function $\left(\mathrm{u}_{\mathrm{i}}\right)$ " to convert the overall dimensional score of a system (e.g., \$, acres, and grades) into a non-dimensional utility value (between 0 and 1) of the system that reflects the decision-maker(s) value.

Step 6: Decide on a weight factor $\left(k_{i}\right)$ for each attribute $\left(i^{\text {th }}\right)$.

Step 7: Calculate the overall score of the system as " $\sum \mathrm{k}_{\mathrm{i}} \mathrm{u}_{\mathrm{i}}$ " (multi-attribute utility function).

Step 8: Use optimization technique to evaluate all possible systems and to find the best system for a specific site. Once all possible systems have been evaluated, the system with the highest overall score is the best system.

Step 9: Conduct a sensitivity analysis to examine the impacts of possible changes in the attribute scores, weight factors, and utility functions on the optimal system.

Step 10: Suggest a small number of systems that should be attractive for a given site. 
By performing the procedure illustrated above, this research provides a quantitative basis for suggesting appropriate drilling systems, explicitly evaluates alternatives against selected criteria, uses the best available information - both expert knowledge and data in a coherent and logical way, and can help decision-makers with their choices of EFD technology for a given situation and best meet the goals of those involved. How to evaluate all possible systems with given information is fully described in Section 5.2 through Section 5.9.

\subsection{Identify the Main Subsystems, Subsets, and Technologies within Each Subset for the EFD Operation}

Four main subsystems and thirteen subsets including over hundred technologies have been identified for the EFD operation as shown in Figure 5-1 and Figure 5-2.

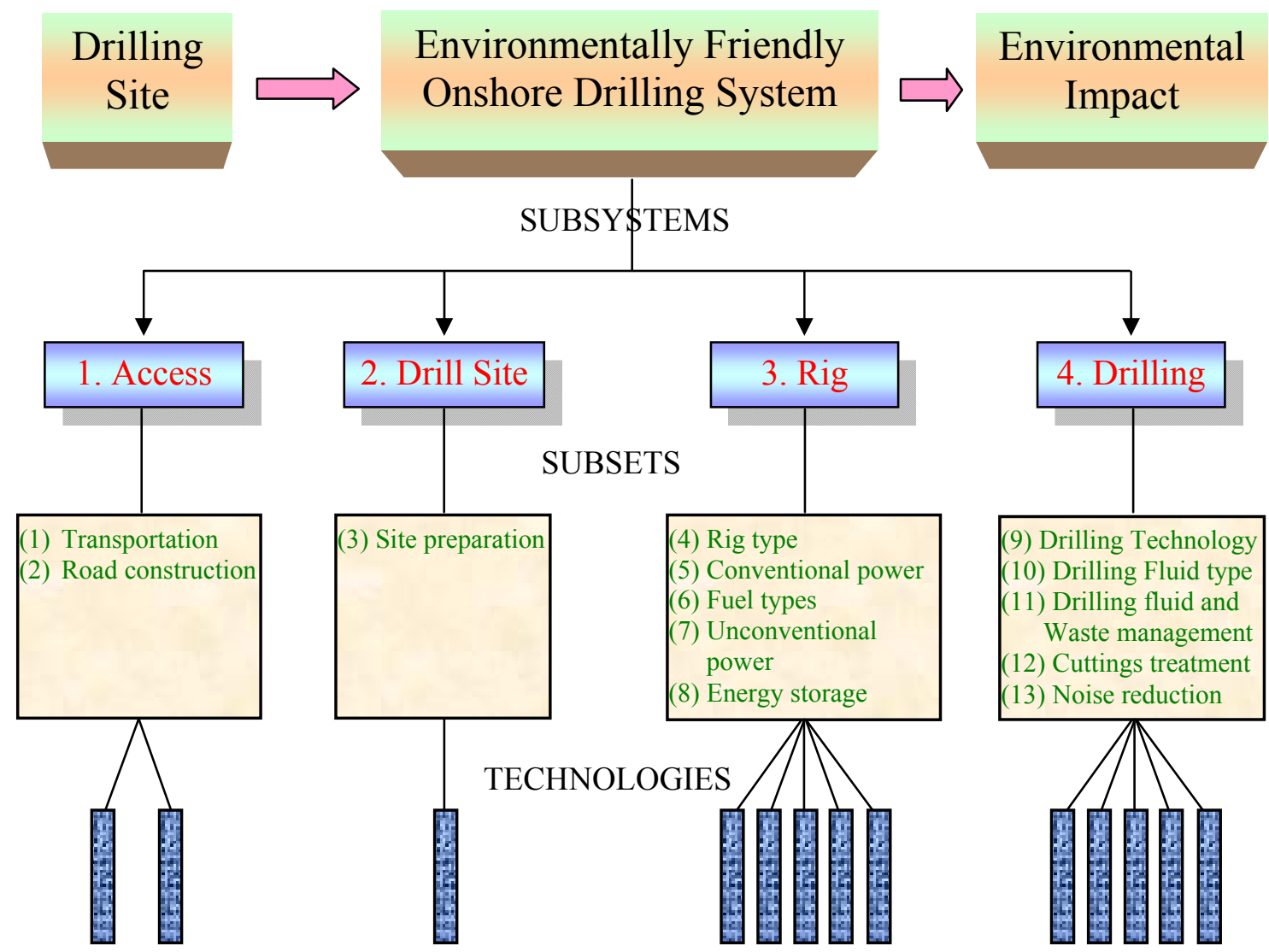

Figure 5-1. The structure of the EFD operation 


\begin{tabular}{|c|c|c|c|}
\hline (1) Transportation & (3) Site Preparation & (4) Rig Type & (9) Drilling Technology \\
\hline Conventional diesel truck & Aluminum modules + bored piles & Barge rig & Overbalanced drilling \\
\hline $\begin{array}{l}\text { Conventional diesel truck } \\
\text { w/noise suppressor }\end{array}$ & Aluminum modules + driven piles & Casing drilling rig & $\begin{array}{l}\text { Overbalanced drilling } \\
\text { w/noise suppressor }\end{array}$ \\
\hline $\begin{array}{l}\text { Low sulphur diesel truck } \\
\text { w/tier III engine }\end{array}$ & Aluminum modules + spread footing & Coiled tubing rig & Underbalanced drilling \\
\hline $\begin{array}{l}\text { Low sulphur diesel truck } \\
\text { w/tier III engine, } \\
\text { w/noise suppressor }\end{array}$ & Board location & Flex rig & $\begin{array}{l}\text { Underbalanced drilling } \\
\text { w/noise suppressor }\end{array}$ \\
\hline Helicopter & Caliche & Ideal rig & Managed pressure drilling \\
\hline Helicopter w/noise suppressor & Cement pad & LOC250 (CWD) & $\begin{array}{l}\text { Managed pressure drilling } \\
\text { w/noise suppressor }\end{array}$ \\
\hline Hovercraft & Compacted fill material & Rapid Rig & (10) Drilling Fluid Type \\
\hline Hovercraft w/noise suppressor & Compacted native material & Traditional older vintage rig & Aerated muds (inject gas) \\
\hline Mattracks & DURA-BASE Composite Mat & Trailer mounted rig & Dry gas and mist \\
\hline Mattracks w/noise suppressor & Gravel pad & Truck mounted rig & Energized and foam \\
\hline Rolligon & Ice pad (Arctic) & (5) Conventional Power & Oil-based muds \\
\hline Rolligon w/noise suppressor & Just level out & Internal combustion engine & Synthetic-based muds \\
\hline (2) Road Construction & Multiple well pad $<10 \mathrm{ft}$. well spacin & $\begin{array}{l}\text { Internal combustion engine } \\
\text { w/noise suppressor }\end{array}$ & Visco-elastic fluids \\
\hline Board road & Multiple well pad 10-20 ft. well spac & Internal combustion engine w/SCR & Water-based muds \\
\hline Bridge decks (small wetland) & Piling & $\begin{array}{l}\text { Internal combustion engine w/SCR, } \\
\text { w/noise suppressor }\end{array}$ & $\begin{array}{l}\text { (11) Drilling Fluid and } \\
\text { Waste Management }\end{array}$ \\
\hline Cable \& wood & Recycle drill cuttings & Large scale utility turbines & $\begin{array}{l}\text { Closed loop + containers } \\
+ \text { solid control equipment }\end{array}$ \\
\hline Corduroy crossings & & $\begin{array}{l}\text { Large scale utility turbines } \\
\text { w/noise suppressor }\end{array}$ & Lined reserve pit + solid control \\
\hline DURA-BASE Composite Mat & & Lean-burn natural gas engines & Open reserve pit + solid control \\
\hline Expanded metal grating & & $\begin{array}{l}\text { Lean-burn natural gas engines } \\
\text { w/noise suppressor }\end{array}$ & (12) Cuttings Treatment \\
\hline Gravel roads & & (6) Fuel Type & Bioremediation \\
\hline Hexadeck & & Bi-fuel system concept & $\begin{array}{l}\text { Chemical fixation and } \\
\text { solidification (CFS) }\end{array}$ \\
\hline Pole rail crossings & & Biodiesel & Co-composting \\
\hline PVC or HDPE pipe mats & & Conventional diesel & Composting \\
\hline Recycled drill cutting road base & & Low sulphur diesel & Cuttings injection \\
\hline Roll-out road & & Synthetic fuels & Evaporation and burial onsite \\
\hline Roverdeck & & Bio-gas & In-situ vitrification \\
\hline Tire mats & & Natural gas & Land-spreading \\
\hline Wood mats & & (7) Unconv. Power & Thermal desorption \\
\hline \multirow[t]{11}{*}{ Wood panels } & & Electric power from grid & Plasma arc \\
\hline & & Fuel cells & (13) Noise Reduction \\
\hline & & Photovoltaic & Construct buildings \\
\hline & & Wind turbines & Construct walls \\
\hline & & $\mathrm{N} / \mathrm{A}$ & N/A \\
\hline & & (8) Energy Storage & \\
\hline & & Battery & \\
\hline & & Capacitor banks & \\
\hline & & Electrolysis to hydrogen & \\
\hline & & Flywheels & \\
\hline & & $\mathrm{N} / \mathrm{A}$ & \\
\hline
\end{tabular}

Figure 5-2. List of technologies within each subset 
Although the technology list shown in Figure 5-2 is not an exhaustive search, what it shows is the current and state of the art technologies for onshore operations. The following Figure 5-3 shows an example of the EFD technology selection. Each path through the subset tables represents one example of a possible EFD system.

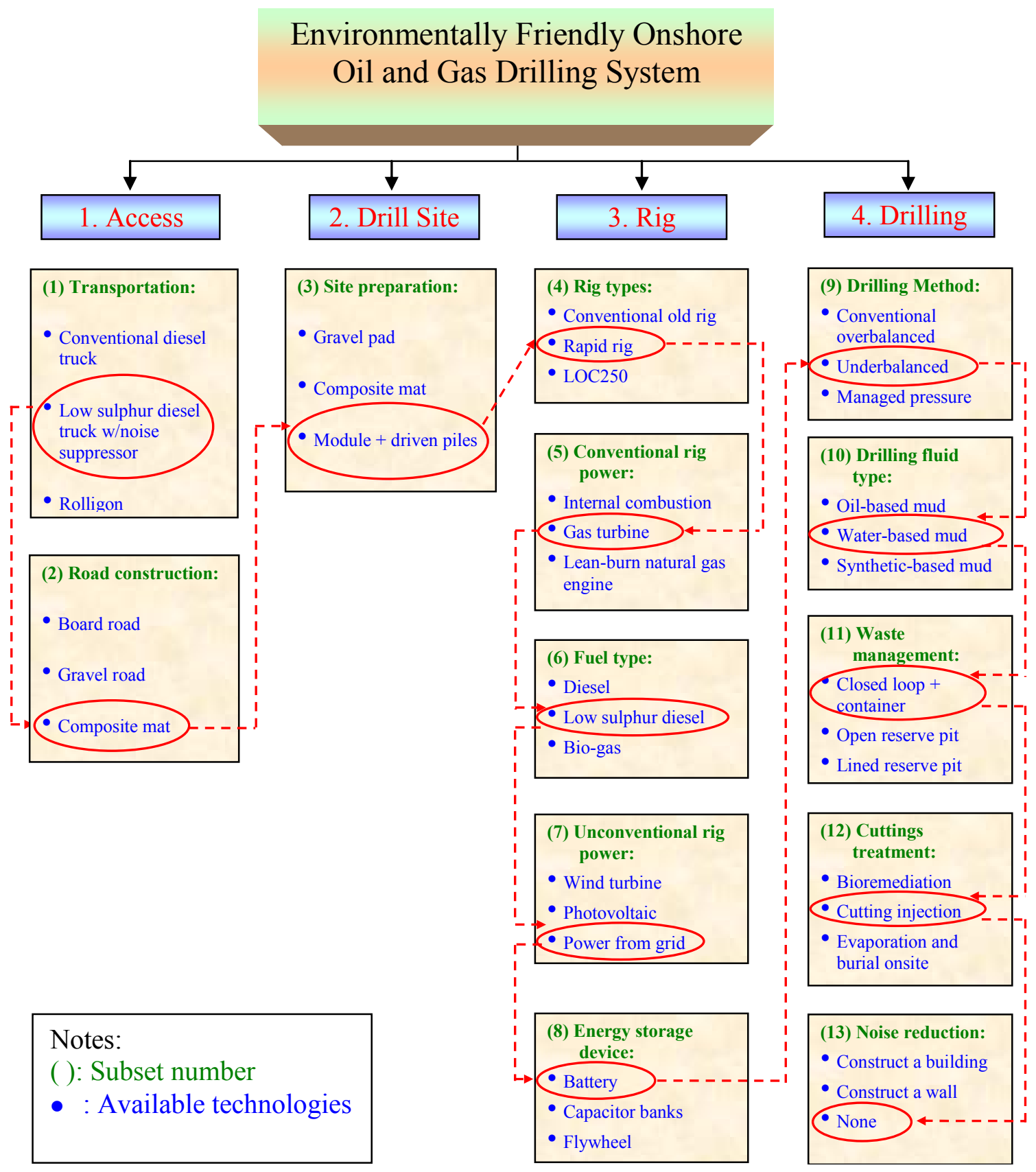

Figure 5-3. An example of the EFD technology selection 


\subsection{Develop Attributes and Attribute Scales}

An attribute is one of the parameters considered in the evaluation of the system (e.g., cost, land area, emission, perception, and safety). Each attribute has an attribute scale used to score the technology on how well it meets the objective for this attribute (e.g., minimizes cost, footprint, and emission while maximizes positive perception and safety value)

In order to evaluate available technologies for onshore oil and gas drilling projects against each attribute, attribute scales that explicitly described their possible impacts on a project need to be specified (Keeney and Raiffa 1976). Nine attributes and their draft scales as defined by EFD subject matter experts are given in this section. These attributes should be both comprehensive and measurable (Keeney and Raiffa 1976) but it should be noted that the attributes do not need to be directly measurable

entity (i.e., $\$$ and acres). Constructed attributes (i.e., perception) can be, and often are, used instead (Keeney 1992). The attribute scales developed in this section are draft scales and thus further interaction with appropriate experts would be valuable in revising these scales.

1. Total cost $\left(\mathrm{x}_{1}\right)=$ if purchasing a technology, then it is suggested to assume the resale value of the technology so as to estimate the total expenditure for the technology during the drilling operation. In the case study described in Section 6, the resale value is assumed as $80 \%$ of the original technology cost. On the other hand, if renting a technology, then a daily rate of the technology is required to estimate the total expenditure during the drilling operation; minimizing cost is preferred.

2. Ecological footprint $\left(\mathrm{x}_{2}\right)=$ the total used land area in acres; minimizing ecological footprint is preferred.

3. Emissions of Environmental Protection Agency (EPA) and state regulated air pollutants $\left(\mathrm{x}_{3}\right)=$ it is suggested by an environmental expert to consider three air contaminants (i.e., CO, Nox, and PM) for this attribute. The relative importance of those contaminants is CO (20\%), Nox (40\%), and PM (40\%) as shown in Table 5-1. Table 5-1 shows an example of how to calculate air emission score for each 
technology. First, estimate three contaminants' real value for each technology in pounds per operating hour. Second, in order to get an overall air emission score for each technology, transform each contaminant's score into a non-dimensional score (U-value) between 0 and 1 using the proportional scoring approach, (x - worst score $) /($ best score - worst score). In this calculation, the best and worst scores should be obtained among all possible technologies being used. Finally, calculate the overall air emission score of a technology as $\sum \mathrm{k}_{\mathrm{i}} \mathrm{u}_{\mathrm{i}}$ (where $\mathrm{k}_{\mathrm{i}}$ is a weight factor for each air contaminant, $\mathrm{u}_{\mathrm{i}}$ is a non-dimensional score for each contaminant). This approach allows the decision-maker to make all air emission scores uniform and comparable; minimizing air emissions is preferred.

Table 5-1. An example of air emission score calculation

\begin{tabular}{|c|c|c|c|c|c|}
\hline \multirow{2}{*}{ Technologies } & \multirow{2}{*}{ Unit } & 0.2 & 0.4 & 0.4 & \multirow{2}{*}{$\begin{array}{l}\text { Overall } \\
\text { score }\end{array}$} \\
\hline & & $\mathrm{CO}$ & $\mathrm{NO}_{\mathrm{x}}$ & PM & \\
\hline \multirow{5}{*}{ Gravel road: Diesel truck + dust } & (gram/hp-hr) & 15.5 & 4 & 0.1 & \multirow{5}{*}{$\underline{0.566}$} \\
\hline & (lb/hp-hr) & 0.03418 & 0.00882 & 0.00022 & \\
\hline & (lb/hr)/unit & 10.253 & 2.646 & 0.216 & \\
\hline & (lb/operating) & 3250.280 & 838.782 & 68.520 & \\
\hline & U-value & \begin{tabular}{l|l}
0.000 \\
\end{tabular} & \begin{tabular}{l|l}
0.822 \\
\end{tabular} & 0.593 & \\
\hline \multirow{5}{*}{$\begin{array}{l}\text { Composite mat: Low sulphur diesel } \\
\text { truck w/tier III engine }\end{array}$} & (gram/hp-hr) & 15.5 & 0.2 & 0.01 & \multirow{5}{*}{$\underline{0.976}$} \\
\hline & (lb/hp-hr) & 0.03418 & 0.00044 & 0.00002 & \\
\hline & $(\mathrm{lb} / \mathrm{hr}) / \mathrm{unit}$ & 10.253 & 0.132 & 0.007 & \\
\hline & (lb/operating) & 369.117 & 4.763 & 0.238 & \\
\hline & U-value & 0.886 & 0.999 & 0.999 & \\
\hline \multirow{5}{*}{ Internal Combustion Engine } & (lb/MWh) & 6.2 & 21.8 & 0.78 & \multirow{5}{*}{$\underline{0.118}$} \\
\hline & $(\mathrm{lb} / \mathrm{hr}) /$ unit & 6.200 & 21.800 & 0.780 & \\
\hline & $(\mathrm{lb} / \mathrm{hr})^{*}$ portion & 6.200 & 21.800 & 0.780 & \\
\hline & (lb/operating) & 1339.200 & 4708.800 & 168.480 & \\
\hline & U-value & 0.588 & 0.000 & 0.000 & \\
\hline \multirow{5}{*}{ Internal Combustion Engine with SCR } & (lb/MWh) & 6.2 & 4.7 & 0.78 & \multirow{5}{*}{$\underline{0.431}$} \\
\hline & $(\mathrm{lb} / \mathrm{hr}) /$ unit & 6.200 & 4.700 & 0.780 & \\
\hline & $(\mathrm{lb} / \mathrm{hr})^{*}$ portion & 6.200 & 4.700 & 0.780 & \\
\hline & (lb/operating) & 1339.200 & 1015.200 & 168.480 & \\
\hline & U-value & 0.588 & 0.784 & 0.000 & \\
\hline \multirow{5}{*}{ Lean-burn natural gas engine } & (lb/MWh) & 5 & 2.2 & 0.03 & \multirow{5}{*}{$\underline{0.878}$} \\
\hline & $(\mathrm{lb} / \mathrm{hr}) / \mathrm{unit}$ & 5.000 & 2.200 & 0.030 & \\
\hline & $(\mathrm{lb} / \mathrm{hr})^{*}$ portion & 5.000 & 2.200 & 0.030 & \\
\hline & (lb/operating) & 1080.000 & 475.200 & 6.480 & \\
\hline & U-value & 0.668 & 0.899 & 0.962 & \\
\hline \multirow{5}{*}{ Power from grid } & (lb/MWh) & 0 & 0 & 0 & \multirow{5}{*}{$\underline{1.000}$} \\
\hline & $(\mathrm{lb} / \mathrm{hr}) / \mathrm{unit}$ & 0.000 & 0.000 & 0.000 & \\
\hline & $(\mathrm{lb} / \mathrm{hr})^{*}$ portion & 0.000 & 0.000 & 0.000 & \\
\hline & (lb/operating) & 0.000 & 0.000 & 0.000 & \\
\hline & U-value & 1.000 & \begin{tabular}{l|l}
1.000 \\
\end{tabular} & 1.000 & \\
\hline
\end{tabular}


It is noted that the linear transformation of emissions to utility is a placeholder and this needs to be reevaluated by experts, perhaps on a case-by-case basis.

4. Emissions of EPA and state regulated solid and liquid pollutants $\left(\mathrm{x}_{4}\right)=$ the ordinal draft scale was constructed by an EFD subject matter expert as shown in Table 5-2; minimizing solid and liquid emissions is preferred.

Table 5-2. Draft attribute scale for solid and liquid emission

\begin{tabular}{|l|l|c|}
\hline $\begin{array}{c}\text { Waste } \\
\text { Management } \\
\text { Technologies }\end{array}$ & \multicolumn{1}{|c|}{ Cuttings treatment } & $\begin{array}{c}\text { Solid/liquid } \\
\text { emission } \\
\text { score }\end{array}$ \\
\hline Closed loop & Cutting injection & 1.00 \\
\hline- & $\begin{array}{l}\text { Bioremediation, Composting, In-situ vitrification, } \\
\text { Land spreading, Plasma arc, Microwave } \\
\text { technology }\end{array}$ & 0.75 \\
\hline Lined reserve pit & Thermal desorption. & 0.50 \\
\hline- & Chemical fixation and solidification & 0.25 \\
\hline Open reserve pit & Evaporation and burial onsite & 0.00 \\
\hline
\end{tabular}

5. Emissions of EPA and state regulated noise pollutants $\left(\mathrm{x}_{5}\right)=$ according to Occupational Safety \& health Administration (OSHA), the eight-hour time-weight average sound level (TWA), in decibels, is recommended as the noise emission's scale. TWA may be computed from the dose, in percent, by means of the formula: TWA $=16.61 \log (\mathrm{D} / 100)+90 . \mathrm{D}$ is the noise dose, in percent: $\mathrm{D}=100 \mathrm{C} / \mathrm{T}$ (where $\mathrm{C}$ is the total length of the work day, in hours, and $\mathrm{T}$ is the reference duration corresponding to the measured sound level, $\mathrm{L}$ in decibel). $\mathrm{T}=8 / 2^{(\mathrm{L}-90) / 5} ;$ minimizing noise emission is preferred.

6. Government, as regulators, perception $\left(\mathrm{x}_{6}\right)=$ the ordinal draft scale was constructed as shown in Table 5-3; maximizing government perception is preferred. 
Table 5-3. Draft attribute scale for government perception

\begin{tabular}{|l|c|}
\hline \multicolumn{1}{|c|}{ Description } & $\begin{array}{c}\text { Perception } \\
\text { score }\end{array}$ \\
\hline $\begin{array}{l}\text { Strongly Support. All parties will encourage its use and are willing to } \\
\text { appropriate funds for the cause. }\end{array}$ & 1.00 \\
\hline $\begin{array}{l}\text { Moderate Support. There is interest from a majority. Its use will be } \\
\text { encouraged, but funds will not be appropriated. }\end{array}$ & 0.75 \\
\hline $\begin{array}{l}\text { Neutrality. All parties are indifferent. There is no resistance, but there } \\
\text { is also no help. }\end{array}$ & 0.50 \\
\hline $\begin{array}{l}\text { Moderate opposition. } \text { Some resistance from the majority. Its use may } \\
\text { be discouraged, but fines or restrictions won't be imposed. }\end{array}$ & 0.25 \\
\hline $\begin{array}{l}\text { Strong opposition. } \text { Strong resistance to its use from all parties. } \\
\text { Restrictions or fines will be set up to eliminate this option. }\end{array}$ & 0.00 \\
\hline
\end{tabular}

7. Industry, as decision-makers, perception $\left(\mathrm{x}_{7}\right)=$ the ordinal draft scale was constructed as shown in Table 5-4; maximizing industry perception is preferred.

Table 5-4. Draft attribute scale for industry perception

\begin{tabular}{|l|c|}
\hline \multicolumn{1}{|c|}{ Description } & $\begin{array}{c}\text { Perception } \\
\text { score }\end{array}$ \\
\hline $\begin{array}{l}\text { Strongly Support. All parties are very interested and willing to invest } \\
\text { for the facility. }\end{array}$ & 1.00 \\
\hline $\begin{array}{l}\text { Moderate Support. All parties are interested but somewhat hesitate to } \\
\text { invest for the facility. }\end{array}$ & 0.75 \\
\hline Neutrality. All parties are indifferent or uninterested. & 0.50 \\
\hline $\begin{array}{l}\text { Moderate opposition. } \\
\text { are indifferent or uninterested. }\end{array}$ & 0.25 \\
\hline Strong opposition. No parties are willing to invest for the facility. & 0.00 \\
\hline
\end{tabular}

8. General public perception $\left(\mathrm{x}_{8}\right)=$ the ordinal draft scale was adapted from Keeney (1992) as shown in Table 5-5; maximizing public perception is preferred. 
Table 5-5. Draft attribute scale for public perception

\begin{tabular}{|l|c|}
\hline \multicolumn{1}{|c|}{ Description } & $\begin{array}{c}\text { Perception } \\
\text { score }\end{array}$ \\
\hline $\begin{array}{l}\text { Support. No groups are opposed to the facility, and at least one group } \\
\text { has organized support for the facility. }\end{array}$ & 1.00 \\
\hline Neutrality. All groups are indifferent or uninterested. & 0.75 \\
\hline $\begin{array}{l}\text { Controversy. One or more groups have organized opposition, } \\
\text { although no groups have action-oriented opposition (for example, } \\
\text { letterwriting, protests, lawsuits). Other groups may either be neutral } \\
\text { or support the facility. }\end{array}$ & 0.50 \\
\hline $\begin{array}{l}\text { Action-oriented opposition. Exactly one group has action-oriented } \\
\text { opposition. The other groups have organized support, indifference, or } \\
\text { organized opposition. }\end{array}$ & 0.25 \\
\hline $\begin{array}{l}\text { Strong action-oriented opposition. Two or more groups have action- } \\
\text { oriented opposition. }\end{array}$ & 0.00 \\
\hline
\end{tabular}

9. Safety value $\left(\mathrm{x}_{9}\right)=$ the ordinal draft scale was constructed referring to OSHA's safety standards as shown in Table 5-6; maximizing safety value is preferred.

Table 5-6. Draft attribute scale for safety value

\begin{tabular}{|l|c|}
\hline \multicolumn{1}{|c|}{ Description } & Safety score \\
\hline Very safe. No hazard associated with a technology. & 1.00 \\
\hline $\begin{array}{l}\text { Safe. It is recommended for workers constructing a technology be } \\
\text { instructed on the hazards of the technology but it is not the } \\
\text { mandatory. No hazard associated with the technology for other } \\
\text { workers. }\end{array}$ & 0.75 \\
\hline $\begin{array}{l}\text { Neutrality. It is recommended for workers in a site be instructed on } \\
\text { the hazards of a technology but it is not the mandatory. }\end{array}$ & 0.50 \\
\hline $\begin{array}{l}\text { Somewhat dangerous. Workers constructing a technology have to be } \\
\text { instructed on the hazards associated with the technology, and it is } \\
\text { recommended for other workers be instructed on the hazards of the } \\
\text { technology, but it is not the mandatory. }\end{array}$ & 0.25 \\
\hline $\begin{array}{l}\text { Very dangerous. Every worker in a site has to be instructed on the } \\
\text { hazards associated with a technology. }\end{array}$ & 0.00 \\
\hline
\end{tabular}

It is required that these attributes and their scales discussed above be revised and restructured, if necessary, through a series of meetings with EFD subject matter experts until the attributes are clearly and meaningfully defined and meet the independence 
assumptions implied by an additive utility function used in this research. A list of EFD experts contacted is available from the author. These nine attributes are assigned to each potential technology. In this research, it is explicitly assumed that the attributes are independent for each possible technology in conducting the technology evaluation over one attribute at a time. In discussion with subject matter experts to date, this assumption seems reasonable.

\subsection{Assign Scores to All Technologies Using the Attribute Scales}

In order to evaluate available technologies with respect to the nine attributes (i.e., $\mathrm{x}_{1}$ through $\mathrm{x}_{9}$ ), EFD subject matter experts' inputs, basic assumptions, and other references are used. Some examples of the cost estimation are shown in Figure 5-4 through Figure 5-9. Moreover, Table 5-7 and Table 5-8 are used to evaluate attribute scores of technologies within subset (5) and subset (7), rig power generation subsets.

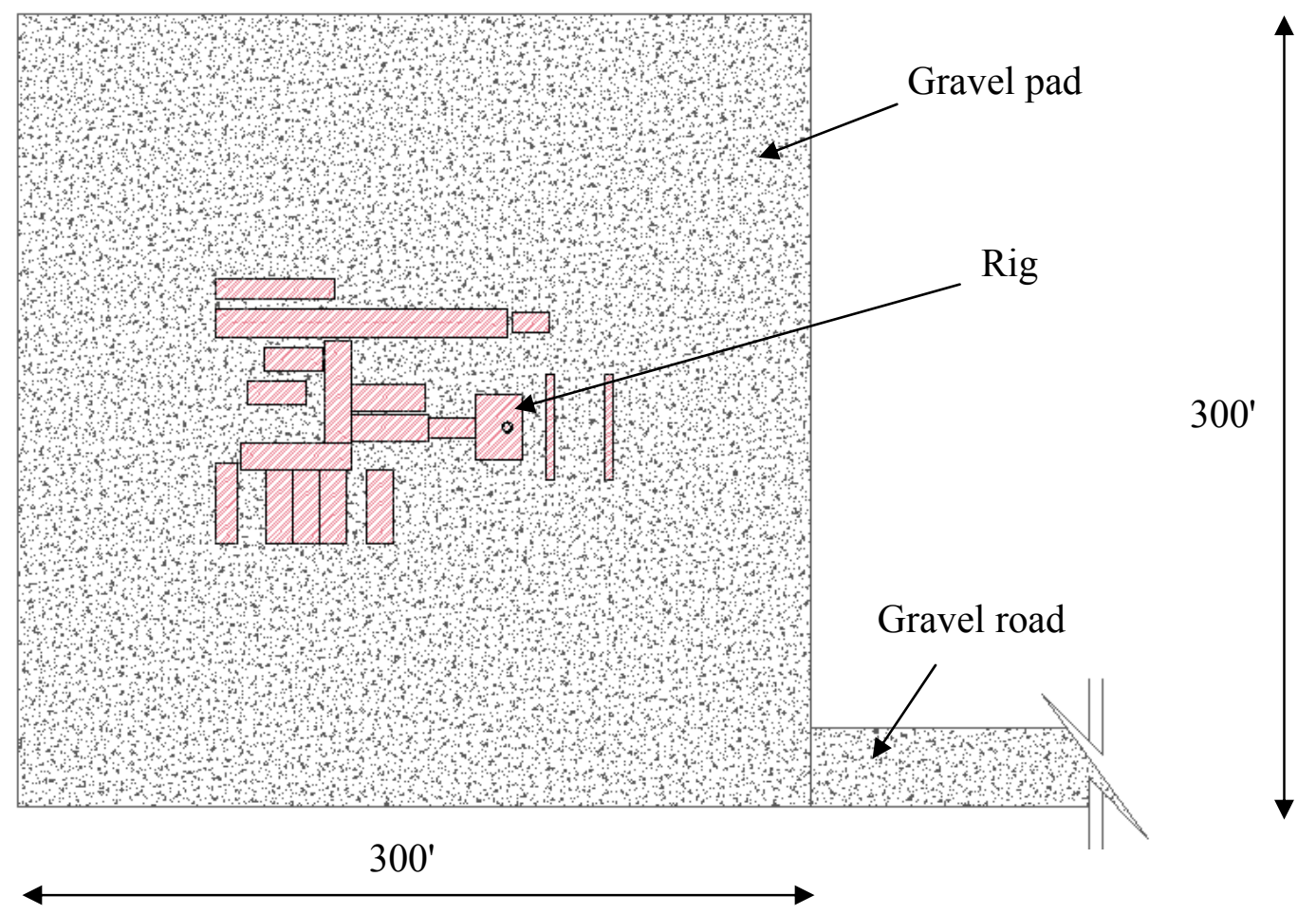

Figure 5-4. A typical layout of a conventional drilling site 


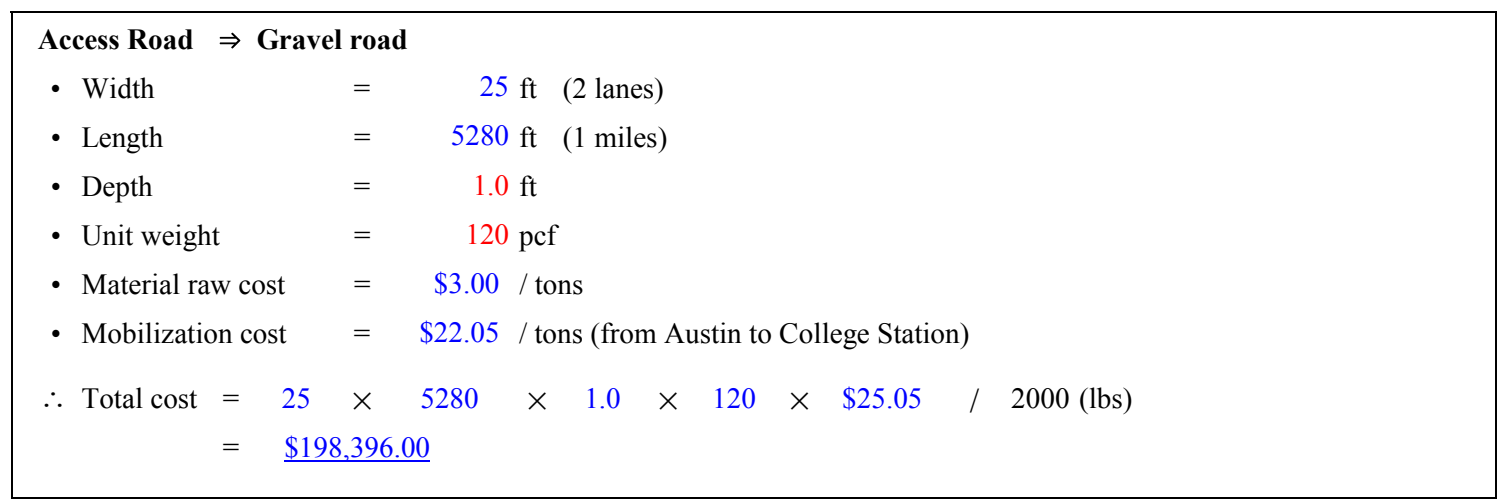

Figure 5-5. Cost estimation of gravel road

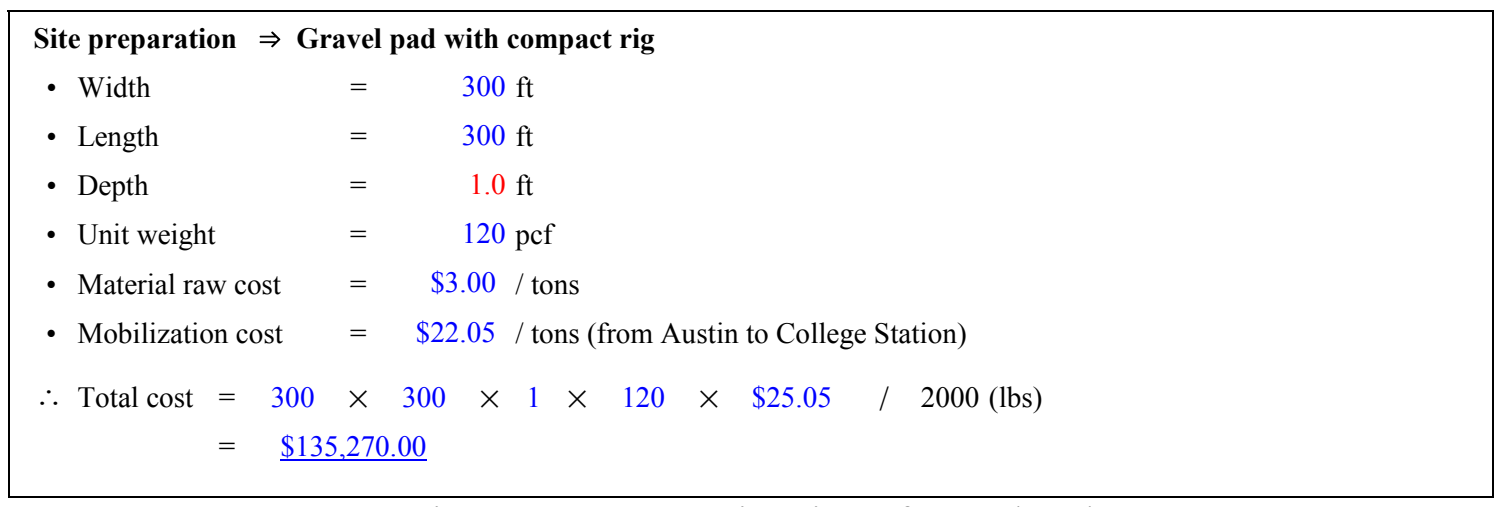

Figure 5-6. Cost estimation of gravel pad

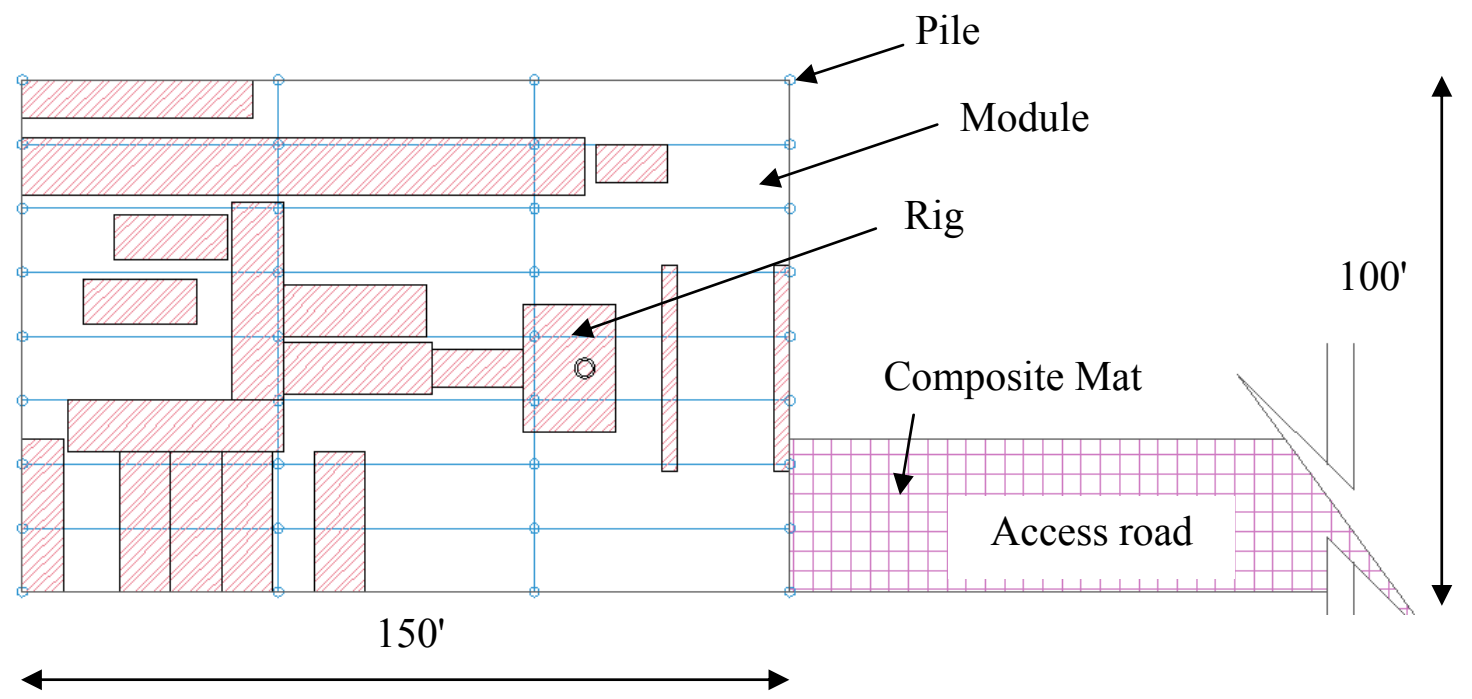

Figure 5-7. A layout of an EFD site 


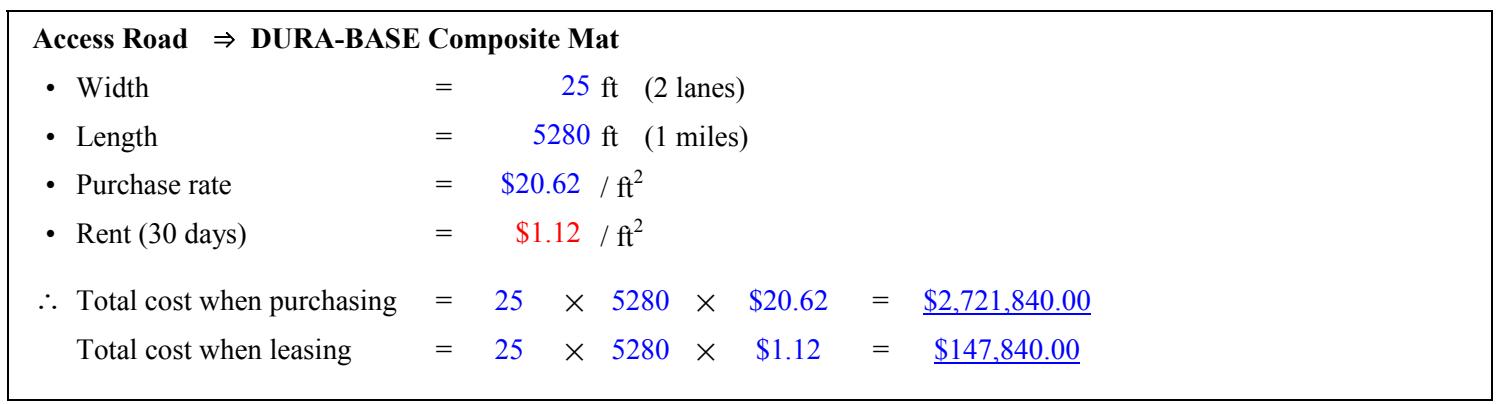

Figure 5-8. Cost estimation of Dura-Base Composite Mat

\section{Site preparation $\rightarrow$ Aluminum modules + Driven piles}

- Aluminum modules:

$>$ Buckets:

$\checkmark \quad$ Dimension $=12.5^{\prime}(\mathrm{W}) \times 50^{\prime}(\mathrm{L}) \times 3.5^{\prime}(\mathrm{D})$

$\checkmark$ Weight $=10,000 \mathrm{lbs}$, each

$>$ Decks:

$\checkmark 6$ " thick aluminum laminates filled with construction form (pre-fabricated)

$\checkmark$ Weight $=12,500 \mathrm{lbs}$, each

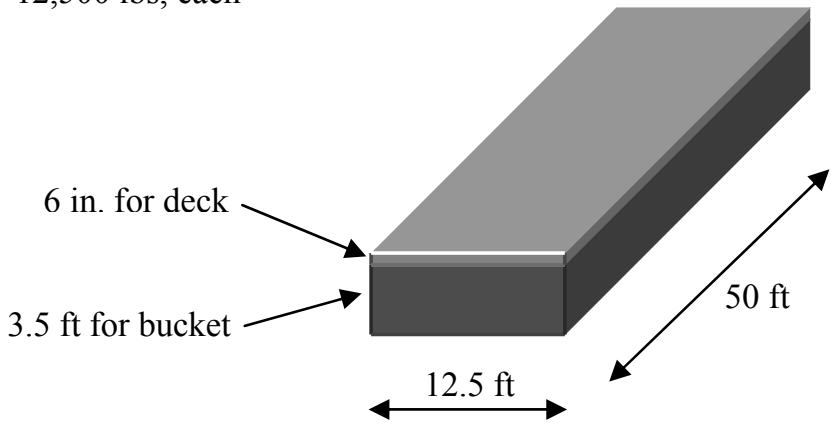

※ Raw material cost of aluminum: $\quad \$ 1.07 \quad / \mathrm{lb}$

$\therefore$ Rough cost of a module:
(1) Raw material cost =
$22500 \times 1.07$
$=\$ 24,075.00$
(2) Fabrication cost
$=\$ 10,000.00 \quad$ (assumed)
(3) Mobilization \& Installation cost

$$
=\$ 10,000.00 \quad \text { (assumed) }
$$
$\Sigma=\$ \underline{\$ 4,075.00}$

- Module cost

$=\$ 44,075 /$ each

- The number of modules

$=\quad 24$ modules

- Pile cost

$=\$ 48,703$ (for 45 piles when using two pile selections)

$\therefore$ Total cost $=\$ 44,075 \times 24+\$ 48,703$

$=\$ 1,106,503.00$

Figure 5-9. Cost estimation of an elevated platform 
total cost of technologies within many subsets. Moreover, selected technologies within subset (5) through subset (8) shown in Figure 5-1 are mutually related each other as shown in Figure 5-11. For example, the number of possible fuel types for a conventional power generation engine varies by what kind of engine is selected, and whether using an energy storage device or not should be dependent on whether an unconventional power generation method is used or not. If it is decided not to use an unconventional power generation method, an energy storage device is not necessarily considered as a subset in the "Rig" subsystem. An example of construction strategy and constraints for the "Rig" subsystem is specified as shown in Figure 5-11. Figure 5-12 shows an example of input spreadsheets used to score technologies in several subsets. The cost, footprint, and emission scores of a technology in subset (1), "Transportation", are not included in the input spreadsheet because those scores are already included as a mobilization part of technologies within other subsets. For example, the cost of gravel road shown in Figure 5-12 includes material, mobilization, and installation costs.

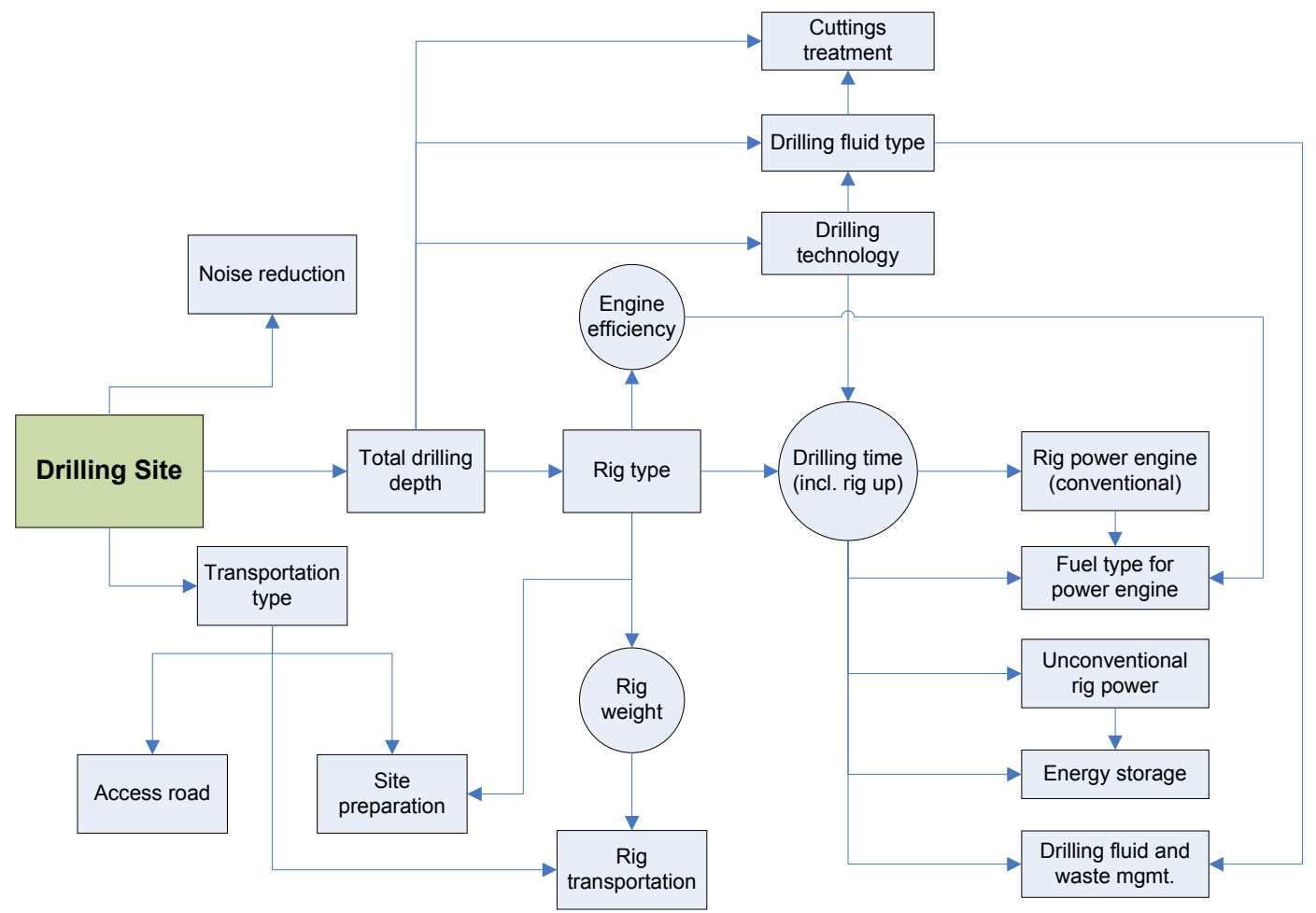

Figure 5-10. Brief influence diagram of a drilling project 


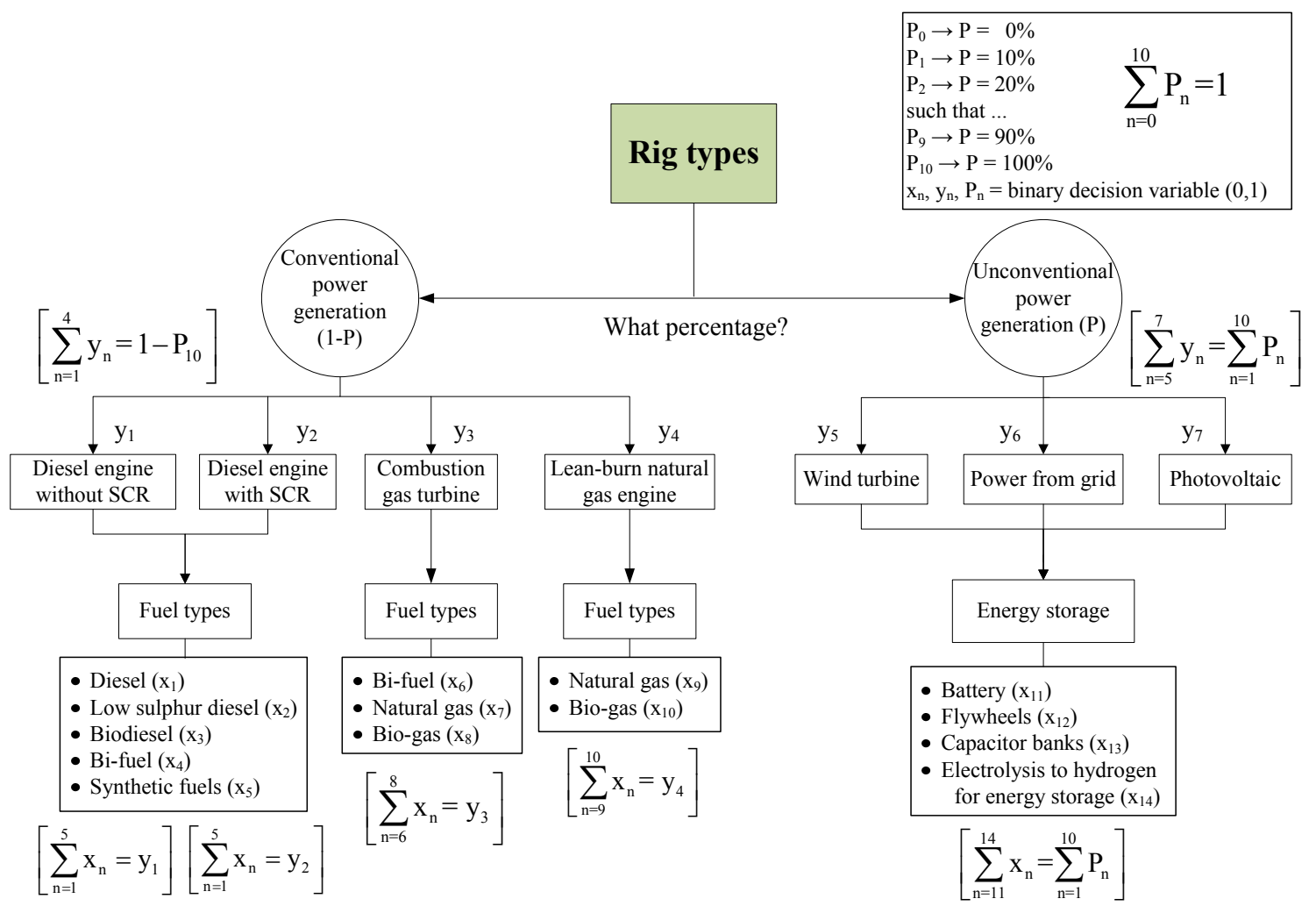

Figure 5-11. Selection procedure and constraints for the "Rig" subsystem

\begin{tabular}{|c|c|c|c|c|c|c|c|c|c|c|}
\hline \multirow{2}{*}{$\begin{array}{l}\text { Sub- } \\
\text { sets }\end{array}$} & \multirow{2}{*}{ Technologies } & \multirow{2}{*}{$\begin{array}{c}\text { Total cost } \\
(\$)\end{array}$} & \multirow{2}{*}{$\begin{array}{c}\text { Ecological } \\
\text { Footprint } \\
\text { (Acres) }\end{array}$} & \multicolumn{3}{|c|}{ Emissions } & \multicolumn{3}{|c|}{ Perceptions } & \multirow{2}{*}{$\begin{array}{l}\text { Safety } \\
\text { Value }\end{array}$} \\
\hline & & & & Air & $\begin{array}{l}\text { Solid\& } \\
\text { Liquid }\end{array}$ & $\begin{array}{l}\text { Noise } \\
\text { (TWA) }\end{array}$ & Gov. & Ind. & Public & \\
\hline \multirow{4}{*}{1} & Coventional diesel truck & & & & & & 0.250 & 1.000 & 0.250 & 0.750 \\
\hline & & & & & & & & & & \\
\hline & MAX & & & & & & 0.250 & 1.000 & 0.250 & 0.750 \\
\hline & MIN & & & & & & 0.250 & 1.000 & 0.250 & 0.750 \\
\hline \multirow{5}{*}{2} & Gravel roads & $\$ 148,500$ & 3.030 & 0.566 & & 98.562 & 0.250 & 1.000 & 0.250 & 0.500 \\
\hline & DURA-BASE from Composite Mat (buy) & $\$ 541,200$ & 1.515 & 0.964 & & 82.870 & 1.000 & 0.500 & 1.000 & 1.000 \\
\hline & DURA-BASE from Composite Mat (rent) & $\$ 132,000$ & 1.515 & 0.964 & & 82.870 & 1.000 & 0.500 & 1.000 & 1.000 \\
\hline & MAX & $\$ 541,200$ & 3.030 & 0.964 & & 98.562 & 1.000 & 1.000 & 1.000 & 1.000 \\
\hline & MIN & $\$ 132,000$ & 1.515 & 0.566 & & 82.870 & 0.250 & 0.500 & 0.250 & 0.500 \\
\hline \multirow{6}{*}{3} & Gravel pad & $\$ 137,813$ & 2.812 & 0.598 & & 98.019 & 0.250 & 1.000 & 0.250 & 0.500 \\
\hline & DURA-BASE from Composite Mat (buy) & $\$ 502,250$ & 1.406 & 0.967 & & 82.242 & 0.750 & 0.750 & 0.750 & 1.000 \\
\hline & DURA-BASE from Composite Mat (rent) & $\$ 122,500$ & 1.406 & 0.967 & & 82.242 & 0.750 & 0.750 & 0.750 & 1.000 \\
\hline & Aluminum modules + driven piles & $\$ 372,408$ & 0.007 & 0.973 & & 97.614 & 1.000 & 0.500 & 1.000 & 0.500 \\
\hline & MAX & $\$ 502,250$ & 2.812 & 0.973 & & 98.019 & 1.000 & 1.000 & 1.000 & 1.000 \\
\hline & MIN & $\$ 122,500$ & 0.007 & 0.598 & & 82.242 & 0.250 & 0.500 & 0.250 & 0.500 \\
\hline \multirow{3}{*}{4} & Traditional older vintage rig & $\$ 220,000$ & & 0.973 & & 78.630 & 0.500 & 1.000 & 0.500 & 0.500 \\
\hline & MAX & $\$ 220,000$ & & 0.973 & & 78.630 & 0.500 & 1.000 & 0.500 & 0.500 \\
\hline & MIN & $\$ 220,000$ & & 0.973 & & 78.630 & 0.500 & 1.000 & 0.500 & 0.500 \\
\hline
\end{tabular}

Figure 5-12. An example of input scores 


\subsection{Calculate the Overall Attribute Score for Each Attribute}

After each technology is evaluated with respect to the nine attributes (i.e., $\mathrm{x}_{1}$ through $\mathrm{x}_{9}$ ), for each attribute, the overall attribute score of a system is calculated by adding the technology scores of the system or selecting the minimum technology score of the system. The addition of individual scores is used for attributes such as cost, footprint, and emission as shown in Eq. (5-1) while the minimum score is used for attributes such as perception and safety as shown in Eq. (5-2). This section elaborates on how to calculate the overall attribute score for each attribute of a system. The overall score on the $i^{\text {th }}$ attribute $\left(\mathrm{X}_{\mathrm{i}}\right)$ is:

$$
\begin{aligned}
& \left.X_{i}=\sum_{n=1}^{N} x_{\text {in }} y_{n} \text { for attribute } x_{1} \text { and } x_{5} \text { (i.e., } i=1 \text { to } 5\right) \\
& X_{i}=\operatorname{Min}\left[x_{\text {in }} y_{n}\right] \text { for attribute } x_{6} \text { through } x_{9} \text { (i.e., } i=3 \text { to 9) }
\end{aligned}
$$

where $\mathrm{n}$ is the index for possible technologies, $\mathrm{N}$ is the number of possible technologies, $i$ is the index for the attributes, $x_{i n}$ is the score of the $n^{\text {th }}$ technology on the $i^{\text {th }}$ attribute, and $\mathrm{y}_{\mathrm{n}}$ is a binary decision variable that is one if $\mathrm{n}^{\text {th }}$ technology is selected and zero if it is not.

The constraint required to consider is:

$$
\sum_{n=1}^{M} y_{n}=1 \text { for each subset except subset (7), (8), and (13) }
$$

where $\mathrm{n}$ is the index for possible technologies, $\mathrm{M}$ is the number of possible technologies within each subset, and $\mathrm{y}_{\mathrm{n}}$ is a binary decision variable.

One technology should be selected within each subset except subset (7), (8), and (13) shown in Figure 5-1. Subset (7), (8), and (13) are optional. Figure 5-13 shows the overall attribute score for each attribute of a system. As can be seen in Figure 5-13, the overall scores of cost $\left(\mathrm{x}_{1}\right)$, footprint $\left(\mathrm{x}_{2}\right)$, and emissions $\left(\mathrm{x}_{3}\right.$ through $\left.\mathrm{x}_{5}\right)$ are calculated by summing the scores of technologies selected within each subset. The overall scores of perceptions $\left(\mathrm{x}_{6}\right.$ through $\left.\mathrm{x}_{8}\right)$, and safety $\left(\mathrm{x}_{9}\right)$, however, are calculated by choosing the worst score among technologies selected within each subset for a system because it is 
suggested that perception and safety values should be considered on the systems level not on the individual technology level.

\begin{tabular}{|c|c|c|c|c|c|c|c|c|c|}
\hline \multirow{2}{*}{$\begin{array}{l}\text { Selected Technologies } \\
\text { in Each Subset }\end{array}$} & \multirow{2}{*}{$\begin{array}{l}\text { Total Cost } \\
\text { (\$) }\end{array}$} & \multirow{2}{*}{$\begin{array}{c}\text { Ecological } \\
\text { Footprint } \\
\text { (Acres) }\end{array}$} & \multicolumn{3}{|c|}{ Emissions } & \multicolumn{3}{|c|}{ Perceptions } & \multirow{2}{*}{$\begin{array}{l}\text { Safety } \\
\text { Value }\end{array}$} \\
\hline & & & Air & $\begin{array}{l}\text { Solid\& } \\
\text { Liquid }\end{array}$ & $\begin{array}{l}\text { Noise } \\
\text { (TWA) }\end{array}$ & Gov. & Ind. & Public & \\
\hline (1) Transportation: Coventional diesel truck & & & & & & 0.250 & 1.000 & 0.250 & 0.750 \\
\hline (2) Road construction: DURA-BASE from Composite Mat (rent) & $\$ 132,000$ & 1.515 & 0.964 & & 82.870 & 1.000 & 0.500 & 1.000 & 1.000 \\
\hline (3) Site preparation: Aluminum modules + driven piles & $\$ 372,408$ & 0.007 & 0.973 & & 97.614 & 1.000 & 0.500 & 1.000 & 0.500 \\
\hline (4) Rig type: Traditional older vintage rig & $\$ 220,000$ & & 0.973 & & 78.630 & 0.500 & 1.000 & 0.500 & 0.500 \\
\hline $\begin{array}{l}\text { (5) Rig power (Conventional): Internal combustion engine w/SCR, } \\
\text { w/noise suppressor }\end{array}$ & $\$ 106,712$ & & 0.488 & & 87.263 & 0.750 & 0.750 & 0.750 & 0.750 \\
\hline (6) Fuel type: Low sulphur diesel & $\$ 88,906$ & & & & & 0.750 & 0.750 & 1.000 & 0.750 \\
\hline (7) Rig power (Unconventional): Electric power from grid (10\%) & $\$ 8,602$ & 0.000 & 1.000 & & 0.000 & 0.500 & 1.000 & 1.000 & 1.000 \\
\hline (8) Energy storage: Flywheels & $\$ 30,000$ & 0.000 & & & & 0.500 & 1.000 & 1.000 & 0.750 \\
\hline (9) Drilling tech.: Conventional overbalanced drilling & $\$ 204,000$ & & & & 116.700 & 1.000 & 0.500 & 0.500 & 0.500 \\
\hline (10) Fluid type: Water-based muds & $\$ 47,940$ & & & & & 1.000 & 1.000 & 1.000 & 1.000 \\
\hline (11) Waste mgmt.: Lined reserve pit + solid control equip.* & $\$ 24,000$ & 0.037 & & 0.500 & & 0.750 & 0.750 & 0.750 & 0.500 \\
\hline (12) Cuttings mgmt:: Cuttings injection & $\$ 60,000$ & & & 1.000 & & 1.000 & 0.500 & 1.000 & 0.750 \\
\hline \multicolumn{10}{|l|}{ (13) Noise reduction: N/A } \\
\hline Overall Attribute Scores ( $\Sigma$ or minimum value) & $\$ 1,294,568$ & 1.559 & 4.398 & 1.500 & 463.077 & 0.250 & 0.500 & 0.250 & 0.500 \\
\hline
\end{tabular}

Figure 5-13. Overall attribute score for each attribute of a system

\subsection{Develop a Utility Function for Each Attribute}

A utility function is a relationship between the dimensional attribute score (e.g., $\$$, acres, and grades) and a non-dimensional number (between 0 and 1) that captures decision-maker preferences. The utility function is used to transform all scores into nondimensional values between 0 and 1 . This allows the decision-maker to make overall attribute score for each attribute uniform and comparable. Once the overall attribute score for each attribute of a system is calculated with respect to the nine attributes (i.e., $\mathrm{x}_{1}$ through $\mathrm{x}_{9}$ ), for each attribute (i) and in order to homogenize the scores, a utility function $\left(\mathrm{u}_{\mathrm{i}}\right)$ needs to be developed to convert the overall dimensional score of a system into a non-dimensional utility value (between 0 and 1) of the system. This section elaborates on how to develop and apply utility functions for this research.

The proportional scoring approach (i.e., linear approach) is mainly suggested in this research to develop single-attribute utility functions because of a lack of expert assessment. This can be revisited as needed based on interactions with EFD subject matter experts. A general formula for the proportional scoring approach is given by: 


$$
\mathrm{u}_{\mathrm{i}}\left(\mathrm{X}_{\mathrm{i}}\right)=\frac{\mathrm{X}_{\mathrm{i}}-\text { Worst Score }}{\text { Best Score }- \text { Worst Score }}
$$

where $\mathrm{X}_{\mathrm{i}}$ is the overall score on the $\mathrm{i}^{\text {th }}$ attribute of a system.

Figure 5-14 shows an example of the utility function curve for the cost attribute. As can be seen in this example, first maximum and minimum values for total cost are obtained. It is found that the range should go from $\$ 0.78$ million dollars to $\$ 1.9$ million dollars, where obviously less total costs are preferred to greater ones. Thus, to remain consistent with the scaling rule where the utility functions ranged from 0 to 1 , it is defined $\mathrm{u}_{1}(\$ 0.78 \mathrm{M})=1$ and $\mathrm{u}_{1}(\$ 1.9 \mathrm{M})=0$. Procedures similar to those described above are also used to assess utility functions for attribute $\mathrm{x}_{2}$ through $\mathrm{x}_{9}$.

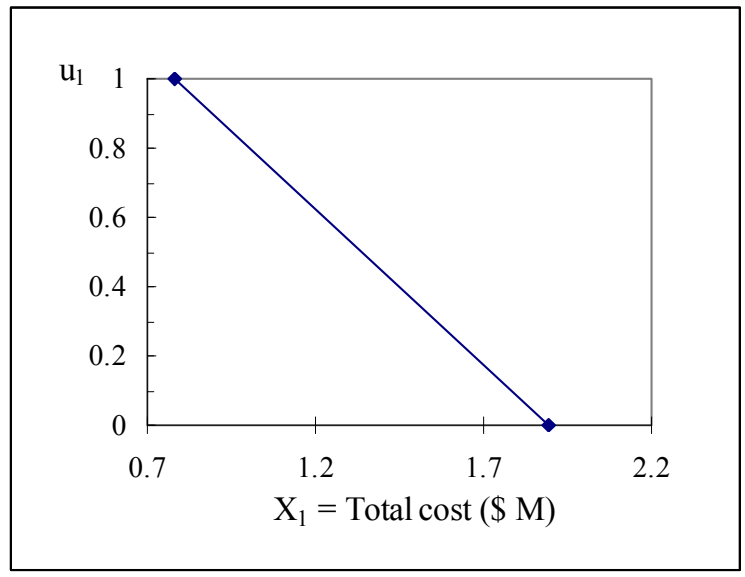

Figure 5-14. The single-attribute utility function curve for cost

In this research, the general shapes of the utility function for each attribute are linear. This implies risk neutrality, but it is very important, before proceeding, to do consistency checks on the reasonableness of the shape of the utility functions (i.e., exponential, linear, etc.) (Keeney and Raiffa 1976). This can be fulfilled by asking additional questions about the decision-maker's preferences, and comparing his/ her responses to the implications of the "fit" utility functions. When they are consistent with each other, the utility functions can be more confidence. When they are inconsistent, on the other hand, the inconsistencies are discussed, and part of all the assessment should be 
repeated (Keeney and Raiffa 1976). Figure 5-15 shows single-attribute utility values of a system.

\begin{tabular}{|c|c|c|c|c|c|c|c|c|c|}
\hline \multirow{2}{*}{$\begin{array}{l}\text { Selected Technologies } \\
\text { in Each Subset }\end{array}$} & \multirow{2}{*}{$\begin{array}{l}\text { Total Cost } \\
\text { (\$) }\end{array}$} & \multirow{2}{*}{$\begin{array}{c}\text { Ecological } \\
\text { Footprint } \\
\text { (Acres) }\end{array}$} & \multicolumn{3}{|c|}{ Emissions } & \multicolumn{3}{|c|}{ Perceptions } & \multirow{2}{*}{$\begin{array}{l}\text { Safety } \\
\text { Value }\end{array}$} \\
\hline & & & Air & $\begin{array}{l}\text { Solid\& } \\
\text { Liquid }\end{array}$ & $\begin{array}{l}\text { Noise } \\
\text { (TWA) }\end{array}$ & Gov. & Ind. & Public & \\
\hline (1) Transportation: Coventional diesel truck & & & & & & 0.250 & 1.000 & 0.250 & 0.750 \\
\hline (2) Road construction: DURA-BASE from Composite Mat (rent) & $\$ 132,000$ & 1.515 & 0.964 & & 82.870 & 1.000 & 0.500 & 1.000 & 1.000 \\
\hline (3) Site preparation: Aluminum modules + driven piles & $\$ 372,408$ & 0.007 & 0.973 & & 97.614 & 1.000 & 0.500 & 1.000 & 0.500 \\
\hline (4) Rig type: Traditional older vintage rig & $\$ 220,000$ & & 0.973 & & 78.630 & 0.500 & 1.000 & 0.500 & 0.500 \\
\hline \begin{tabular}{|l|}
$\begin{array}{l}\text { (5) Rig power (Conventional): Internal combustion engine w/SCR, } \\
\text { w/noise suppressor }\end{array}$ \\
\end{tabular} & $\$ 106,712$ & & 0.488 & & 87.263 & 0.750 & 0.750 & 0.750 & 0.750 \\
\hline (6) Fuel type: Low sulphur diesel & $\$ 88,906$ & & & & & 0.750 & 0.750 & 1.000 & 0.750 \\
\hline (7) Rig power (Unconventional): Electric power from grid (10\%) & $\$ 8,602$ & 0.000 & 1.000 & & 0.000 & 0.500 & 1.000 & 1.000 & 1.000 \\
\hline (8) Energy storage: Flywheels & $\$ 30,000$ & 0.000 & & & & 0.500 & 1.000 & 1.000 & 0.750 \\
\hline (9) Drilling tech.: Conventional overbalanced drilling & $\$ 204,000$ & & & & 116.700 & 1.000 & 0.500 & 0.500 & 0.500 \\
\hline (10) Fluid type: Water-based muds & $\$ 47,940$ & & & & & 1.000 & 1.000 & 1.000 & 1.000 \\
\hline (11) Waste mgmt.: Lined reserve pit + solid control equip.* & $\$ 24,000$ & 0.037 & & 0.500 & & 0.750 & 0.750 & 0.750 & 0.500 \\
\hline (12) Cuttings mgmt.: Cuttings injection & $\$ 60,000$ & & & 1.000 & & 1.000 & 0.500 & 1.000 & 0.750 \\
\hline \multicolumn{10}{|l|}{ (13) Noise reduction: N/A } \\
\hline Overall Attribute Scores ( $\Sigma$ or minimum value) & $\$ 1,294,568$ & 1.559 & 4.398 & 1.500 & 463.077 & 0.250 & 0.500 & 0.250 & 0.500 \\
\hline Single Attribute Utility Values & 0.539 & 0.991 & 0.701 & 0.600 & 0.677 & 0.250 & 0.500 & 0.250 & 0.500 \\
\hline
\end{tabular}

Figure 5-15. Single-attribute utility values of a system

\subsection{Combine Utility Values of Each Attribute into an Overall Utility}

Once each single-attribute utility function $u_{i}\left(X_{i}\right)$ is derived for its attribute measure, these individual utility values are combined in some way into a final utility value. If mutual preferential and utility independence are satisfied, it is possible to define the multi-attribute utility function to the additive form:

$$
\begin{aligned}
U\left(X_{1}, X_{2}, \ldots, X_{I}\right) & =U\left\{u_{1}\left(X_{1}\right), u_{2}\left(X_{2}\right), \ldots, u_{I}\left(X_{I}\right)\right. \\
& =k_{1} u_{1}\left(X_{1}\right)+\ldots+k_{I} u_{I}\left(X_{I}\right)=\sum_{i=1}^{I} k_{i} u_{i}\left(X_{i}\right)
\end{aligned}
$$

where $\mathrm{u}_{\mathrm{i}}\left(\mathrm{X}_{\mathrm{i}}\right)$ is a single-attribute utility function scaled from 0 to $1, \mathrm{k}_{\mathrm{i}}$ is a weight factor for $\mathrm{u}_{\mathrm{i}}\left(\mathrm{X}_{\mathrm{i}}\right)$.

Since it is assumed that there is no interaction between each attribute, all of the weights are positive and they must sum to one (Hardaker 2004). In general, weight factors are decided by decision-makers. Table 5-9 shows an example of assigned weight factor for each attribute. 
Table 5-9. An example of assigned weight factor for each attribute

\begin{tabular}{|l|c|}
\hline \multicolumn{1}{|c|}{ Attributes } & Weights \\
\hline Total cost $\left(\mathrm{x}_{1}\right)$ & 0.40 \\
\hline Footprint $\left(\mathrm{x}_{2}\right)$ & 0.25 \\
\hline Air emission $\left(\mathrm{x}_{3}\right)$ & 0.05 \\
\hline Solid/ liquid emission $\left(\mathrm{x}_{4}\right)$ & 0.05 \\
\hline Noise emission $\left(\mathrm{x}_{5}\right)$ & 0.05 \\
\hline Government perception $\left(\mathrm{x}_{6}\right)$ & 0.05 \\
\hline Industry perception $\left(\mathrm{x}_{7}\right)$ & 0.05 \\
\hline Public perception $\left(\mathrm{x}_{8}\right)$ & 0.05 \\
\hline Safety $\left(\mathrm{x}_{9}\right)$ & 0.05 \\
\hline
\end{tabular}

A multi-attribute utility function of the additive form can be derived in two steps. First, single-attribute utility functions $\mathrm{u}_{\mathrm{i}}\left(\mathrm{X}_{\mathrm{i}}\right)$ of a system are derived for each attribute measure in turn, then these individual utility values are combined into an overall utility value of the system to simplify comparisons with other possible systems. Figure 5-16 shows an example of the multi-attribute utility value of a system with the weighting factors given in Table 5-9.

\begin{tabular}{|c|c|c|c|c|c|c|c|c|c|}
\hline & \multicolumn{9}{|c|}{ Weights $(\Sigma=100 \% \quad \therefore$ O.K! $)$} \\
\hline & $40 \%$ & $25 \%$ & $5 \%$ & $5 \%$ & $5 \%$ & $5 \%$ & $5 \%$ & $5 \%$ & $5 \%$ \\
\hline \multirow{2}{*}{$\begin{array}{l}\text { Selected Technologies } \\
\text { in Each Subset }\end{array}$} & \multirow{2}{*}{$\begin{array}{c}\text { Total Cost } \\
(\$)\end{array}$} & \multirow{2}{*}{$\begin{array}{c}\text { Ecological } \\
\text { Footprint } \\
\text { (Acres) }\end{array}$} & \multicolumn{3}{|c|}{ Emissions } & \multicolumn{3}{|c|}{ Perceptions } & \multirow{2}{*}{$\begin{array}{l}\text { Safety } \\
\text { Value }\end{array}$} \\
\hline & & & Air & $\begin{array}{l}\text { Solid\& } \\
\text { Liquid }\end{array}$ & $\begin{array}{l}\text { Noise } \\
\text { (TWA) }\end{array}$ & Gov. & Ind. & Public & \\
\hline (1) Transportation: Coventional diesel truck & & & & & & 0.250 & 1.000 & 0.250 & 0.750 \\
\hline (2) Road construction: DURA-BASE from Composite Mat (rent) & $\$ 132,000$ & 1.515 & 0.964 & & 82.870 & 1.000 & 0.500 & 1.000 & 1.000 \\
\hline (3) Site preparation: Aluminum modules + driven piles & $\$ 372,408$ & 0.007 & 0.973 & & 97.614 & 1.000 & 0.500 & 1.000 & 0.500 \\
\hline (4) Rig type: Traditional older vintage rig & $\$ 220,000$ & & 0.973 & & 78.630 & 0.500 & 1.000 & 0.500 & 0.500 \\
\hline $\begin{array}{l}\begin{array}{l}\text { (5) Rig power (Conventional): Internal combustion engine w/SCR, } \\
\text { w/noise suppressor }\end{array} \\
\end{array}$ & $\$ 106,712$ & & 0.488 & & 87.263 & 0.750 & 0.750 & 0.750 & 0.750 \\
\hline (6) Fuel type: Low sulphur diesel & $\$ 88,906$ & & & & & 0.750 & 0.750 & 1.000 & 0.750 \\
\hline (7) Rig power (Unconventional): Electric power from grid (10\%) & $\$ 8,602$ & 0.000 & 1.000 & & 0.000 & 0.500 & 1.000 & 1.000 & 1.000 \\
\hline (8) Energy storage: Flywheels & $\$ 30,000$ & 0.000 & & & & 0.500 & 1.000 & 1.000 & 0.750 \\
\hline (9) Drilling tech.: Conventional overbalanced drilling & $\$ 204,000$ & & & & 116.700 & 1.000 & 0.500 & 0.500 & 0.500 \\
\hline (10) Fluid type: Water-based muds & $\$ 47,940$ & & & & & 1.000 & 1.000 & 1.000 & 1.000 \\
\hline (11) Waste mgmt.: Lined reserve pit + solid control equip.* & $\$ 24,000$ & 0.037 & & 0.500 & & 0.750 & 0.750 & 0.750 & 0.500 \\
\hline (12) Cuttings mgmt.: Cuttings injection & $\$ 60,000$ & & & 1.000 & & 1.000 & 0.500 & 1.000 & 0.750 \\
\hline \multicolumn{10}{|l|}{ (13) Noise reduction: N/A } \\
\hline Overall Attribute Scores ( $\Sigma$ or minimum value) & $\$ 1,294,568$ & 1.559 & 4.398 & 1.500 & 463.077 & 0.250 & 0.500 & 0.250 & 0.500 \\
\hline Single Attribute Utility Values & 0.539 & 0.991 & 0.701 & 0.600 & 0.677 & 0.250 & 0.500 & 0.250 & 0.500 \\
\hline$\therefore$ Multi-Attribute Utility Value $=$ & $\underline{0.637}$ & & & & & & & & \\
\hline
\end{tabular}

Figure 5-16. An example of the multi-attribute utility value of a system 


\subsection{Find the Best System}

In this section, an optimization scheme is suggested based on a combination of multi-attribute utility theory and exhaustively enumerating all possible systems to provide a quantitative rationale and suggest the best set of systems according to a set of attributes, with the relative importance of the different attributes defined by the decisionmaker. Since exhaustive search optimization is a simple, practical, and very robust method given the speed of modern computers (Cover et al. 2007), it is used to evaluate all possible systems and to find the 'best' available system that should be particularly attractive for a specific site. Larger problems would likely require more advanced optimization methods. Once all possible systems have been evaluated, the system with the highest overall utility score is the best system with given weighting factors.

After the optimization scheme has given the 'best' system, a sensitivity analysis can be conducted to examine the impact of possible changes in the attribute scores, weight factors, and utility functions on the best system. For example, the weight assigned to the cost attribute shown in Table 5-9 could be changed from the initially assigned value of 0.40 . Since the weighting factors must sum to one in this research, the weights assigned to other attributes are known once a weight assigned to the cost attribute is decided. Conducting a sensitivity analysis for the technology selection process is an importance step because it can give an idea the range of weights over which certain systems should be selected for a specific site (Guikema and Milke 1999).

Note that the final answer needs not be a single system but that a few "optimal" systems which come close to best score can be selected. This may provide some flexibility for the person in charge of the drilling process.

\subsection{Lessons Learned}

Throughout this section, a system optimization approach is suggested based on a combination of multi-attribute utility theory and exhaustive search optimization. This methodology is designed to help decision-makers with their choices of EFD technology in onshore drilling operations. However, the approach used in this research does have 
some limitations. The crucial limitation is that the computational burden of the procedure may become prohibitive for problems with a large number of decision variables. One possible way to resolve this problem in this research is if the analyst can identify subsets that will always select the same technology for any weight combinations, the elimination of those subsets from the original thirteen subsets can significantly reduce computational burdens in future steps.

Moreover, estimating input values for available technologies are a very difficult step to proceed with the quantitative approach suggested in this research. The outcomes of the process should be brought into a question without having the adequate input values. Missing input information introduces additional errors into the analysis because the missing information represents another assumption that must be made to proceed with the analysis (Rehm et al. 2008). Even though many EFD subject matter experts have already participated in this research, more people's inputs and feedbacks are necessary to make the proposed technology selection process easier and quicker. 


\section{A CASE STUDY WITH PRE-SPECIFIED SYSTEMS}

In order to test the technology evaluation protocol proposed in Section 5 in a real site and then to refine the protocol, a case study is conducted in Green Lake at McFaddin, TX. This section describes the results of the case study which provided a more logical and comprehensive approach that maximized the economic and environmental goals of both the landowner and the oil company leaseholder. How to arrive at the optimal drilling system for this site are fully described in this section.

\subsection{Selected Site}

It is assumed that an independent operator is to drill a well on their lease in South Texas in an environmentally sensitive wetland area. The lease extends to the center of Green Lake on the McFaddin Ranch as shown in Figure 6-1. The formation target is the upper Frio sand (Hovorka et al. 2001) at approximately $8500 \mathrm{ft}$ in vertical depth. In order to protect the ranch as much as possible, low impact drilling and utilizing the very best drilling system is extremely important.

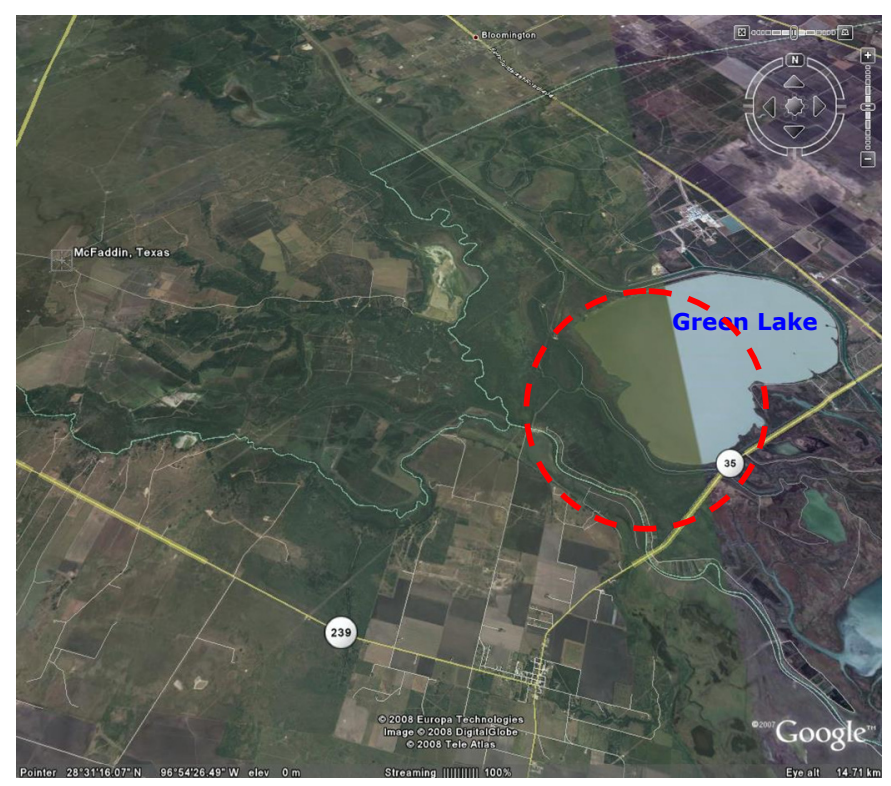

Figure 6-1. A satellite map of Green Lake at McFaddin, Texas 


\subsection{Description of the Pre-Specified Systems}

Three different systems are pre-specified by an EFD expert in order to identify possible drilling technologies for Green Lake drilling site as shown in Table 6-1. A list of EFD experts contacted is available from the author. Although the technology list shown in Table 6-1 is not an exhaustive search, what it shows is the current and state of the art technologies for onshore oil and gas drilling operations.

Table 6-1. Pre-specified drilling systems

\begin{tabular}{|c|c|c|c|}
\hline Subsets & $\begin{array}{l}\text { 1. Conventional } \\
\text { Drilling }\end{array}$ & $\begin{array}{c}\text { 2. Moderately } \\
\text { Improved Drilling }\end{array}$ & 3. EFD in 5 years \\
\hline (1) Transportation & $\begin{array}{l}\text { Conventional diesel } \\
\text { truck }\end{array}$ & $\begin{array}{l}\text { Low sulphur diesel } \\
\text { truck w/tier III engine, } \\
\text { w/noise suppressor }\end{array}$ & $\begin{array}{l}\text { Low sulphur diesel } \\
\text { truck w/tier III engine, } \\
\text { w/noise suppressor }\end{array}$ \\
\hline $\begin{array}{l}\text { (2) Road } \\
\text { construction }\end{array}$ & Gravel road & $\begin{array}{l}\text { DURA-BASE from } \\
\text { Composite Mat (rent) }\end{array}$ & $\begin{array}{l}\text { DURA-BASE from } \\
\text { Composite Mat (rent) }\end{array}$ \\
\hline (3) Site preparation & Gravel pad & $\begin{array}{l}\text { DURA-BASE from } \\
\text { Composite Mat (rent) }\end{array}$ & $\begin{array}{l}\text { Aluminum modules }+ \\
\text { driven piles (elevated } \\
\text { platform) }\end{array}$ \\
\hline (4) Rig type & $\begin{array}{l}\text { Traditional older } \\
\text { vintage rig }\end{array}$ & Rapid Rig & LOC250 (CWD) \\
\hline $\begin{array}{l}\text { (5) Conventional } \\
\text { rig power } \\
\text { engine } \\
\end{array}$ & $\begin{array}{l}\text { Internal combustion } \\
\text { engine }\end{array}$ & $\begin{array}{l}\text { Internal combustion } \\
\text { engine } \mathrm{w} / \mathrm{SCR} \text {, } \\
\text { w/noise suppressor }\end{array}$ & $\begin{array}{l}\text { Lean-burn natural gas } \\
\text { engines } \mathrm{w} / \text { noise } \\
\text { suppressor }\end{array}$ \\
\hline (6) Fuel type & Conventional diesel & Low sulphur diesel & Natural gas \\
\hline $\begin{array}{l}\text { (7) Unconventional } \\
\text { rig power } \\
\text { generation } \\
\end{array}$ & None & $\begin{array}{l}\text { Electric power from } \\
\text { grid }(10 \%)\end{array}$ & $\begin{array}{l}\text { Electric power from } \\
\text { grid }(30 \%)\end{array}$ \\
\hline (8) Energy storage & None & Flywheel & Flywheel \\
\hline $\begin{array}{l}\text { (9) Drilling } \\
\text { technology }\end{array}$ & $\begin{array}{l}\text { Conventional } \\
\text { overbalanced drilling }\end{array}$ & $\begin{array}{l}\text { Underbalanced drilling } \\
\text { w/noise suppressor }\end{array}$ & $\begin{array}{l}\text { Managed pressure } \\
\text { drilling w/noise } \\
\text { suppressor }\end{array}$ \\
\hline (10) Fluid type & Water-based muds & Water-based muds & Water-based muds \\
\hline $\begin{array}{l}\text { (11) Drilling fluid } \\
\text { and waste } \\
\text { management }\end{array}$ & $\begin{array}{l}\text { Lined reserve pit }+ \\
\text { solid control } \\
\text { equipment }\end{array}$ & $\begin{array}{l}\text { Closed loop }+ \\
\text { containers }+ \text { solid } \\
\text { control equipment }\end{array}$ & $\begin{array}{l}\text { Closed loop }+ \\
\text { containers }+ \text { solid } \\
\text { control equipment }\end{array}$ \\
\hline $\begin{array}{l}\text { (12) Cuttings } \\
\text { treatment }\end{array}$ & Cuttings injection & Cuttings injection & $\begin{array}{l}\text { Chemical fixation and } \\
\text { solidification (CFS) }\end{array}$ \\
\hline $\begin{array}{l}\text { (13) } \begin{array}{l}\text { Noise } \\
\text { reduction }\end{array} \\
\end{array}$ & None & None & None \\
\hline
\end{tabular}




\subsection{Calculate Overall Utility Values of the Pre-Specified Systems}

In order to calculate the overall utility score, the procedure described in Section 5 is required to be implemented. EFD subject matter experts' inputs, basic assumptions, and other references are used to evaluate available technologies with respect to the nine attributes (i.e., $\mathrm{x}_{1}$ through $\mathrm{x}_{9}$ ). Figure 6-2 shows the basic assumptions used in this case study and key input variables which are the most influence factors for input values of technologies.

\section{Basic Assumptions}

- Power consumption (peak):

$1 \mathrm{MW}$

- Access road width:

$25 \mathrm{ft}$ (2 lanes)

- Access road length:

1 miles

- Width of drilling site:

$350 \mathrm{ft}$ (conventional rig + pad)

$300 \mathrm{ft}$ (compact rig + pad)

$200 \mathrm{ft}$ (conventional rig + modules + piles)

$150 \mathrm{ft}$ (compact rig + modules + piles)

- Length of drilling site: $\quad 350 \mathrm{ft}($ conventional rig + pad)

$300 \mathrm{ft}$ (compact rig + pad)

$125 \mathrm{ft}$ (conventional rig + modules + piles)

$100 \mathrm{ft}$ (compact rig + modules + piles)

\section{Key Influence variables}

- Transportation type:

Coventional diesel truck

- Rig Type:

LOC250 (CWD)

- Engine Type:

Internal combustion engine

- Drilling Type:

Conventional overbalanced drilling

- Noise reduction type:

$\mathrm{N} / \mathrm{A}$

- Proportion of unconventional power: $\quad 30.0 \%$

- Resale value:

$80.0 \%$

- Drilling Time:

9.0 days

- Move/Rig up:

1.0 days

- No. of wells:

1 wells

Figure 6-2. Basic assumptions and key influence variables 
The influence diagram for this drilling site shown in Figure 6-3 should be considered before estimating attribute scores of technologies because attribute scores of a technology can be dependent on key influence variables described in Figure 6-2. For example, different rig type causes the variation of total drilling time and the total drilling time varies total cost of technologies within many subsets.

In this case study, the range of unconventional power usage is varied from $0 \%$ to $30 \%$ of total power usage. The construction strategy and constraints of the "Rig" subsystem are specified as shown in Figure 6-4. Figure 6-5 shows an example of input spreadsheets used to score technologies in several subsets. The cost, footprint, and emission scores of a technology in subset (1), "Transportation", are not included in the input spreadsheet because those scores are already included as a mobilization part of technologies within other subsets. For example, the cost of gravel road shown in Figure 6-5 includes material, mobilization, and installation costs. More detailed input values of available technologies can be found in APPENDIX B.

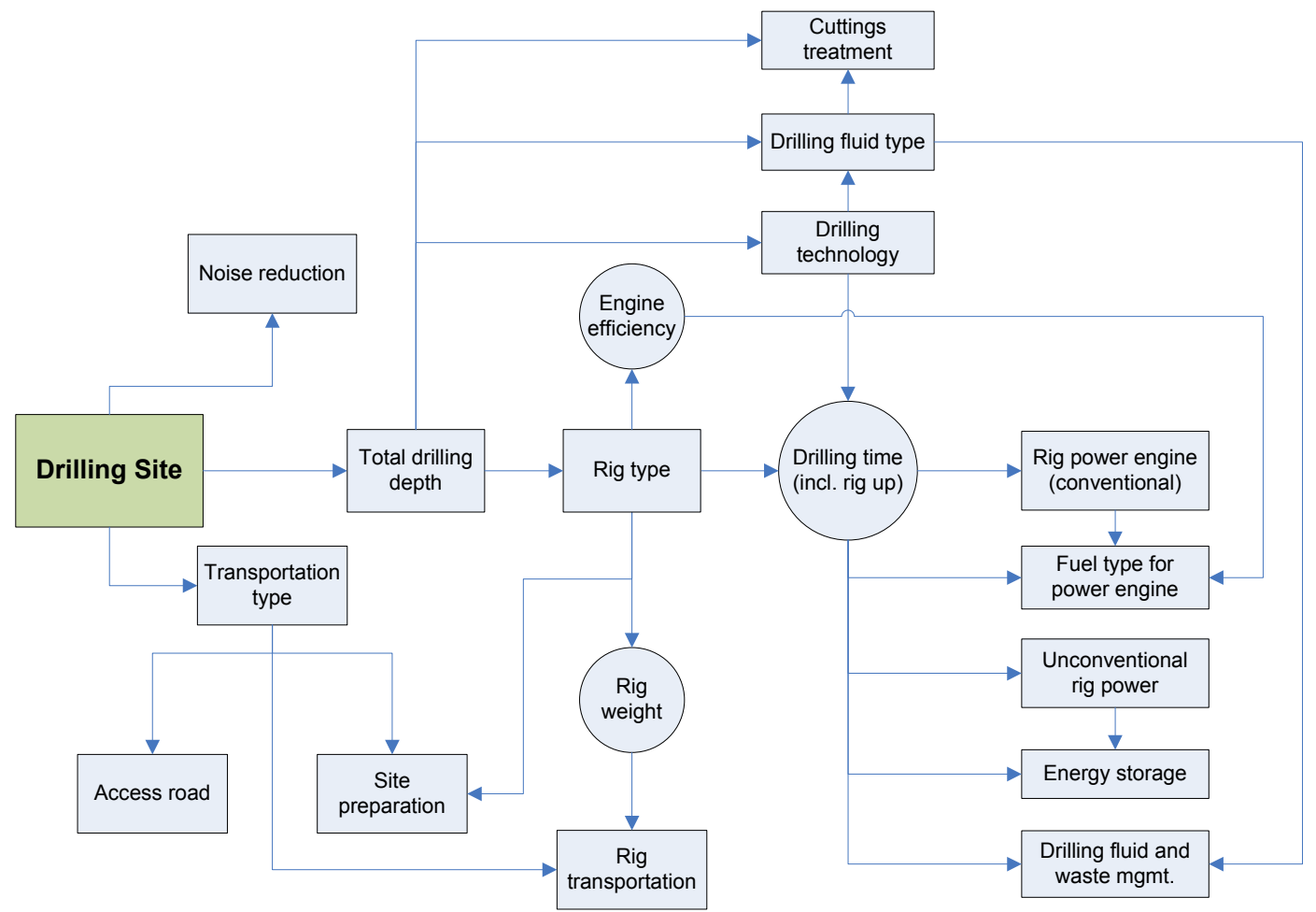

Figure 6-3. Influence diagram for the drilling site of the case study 


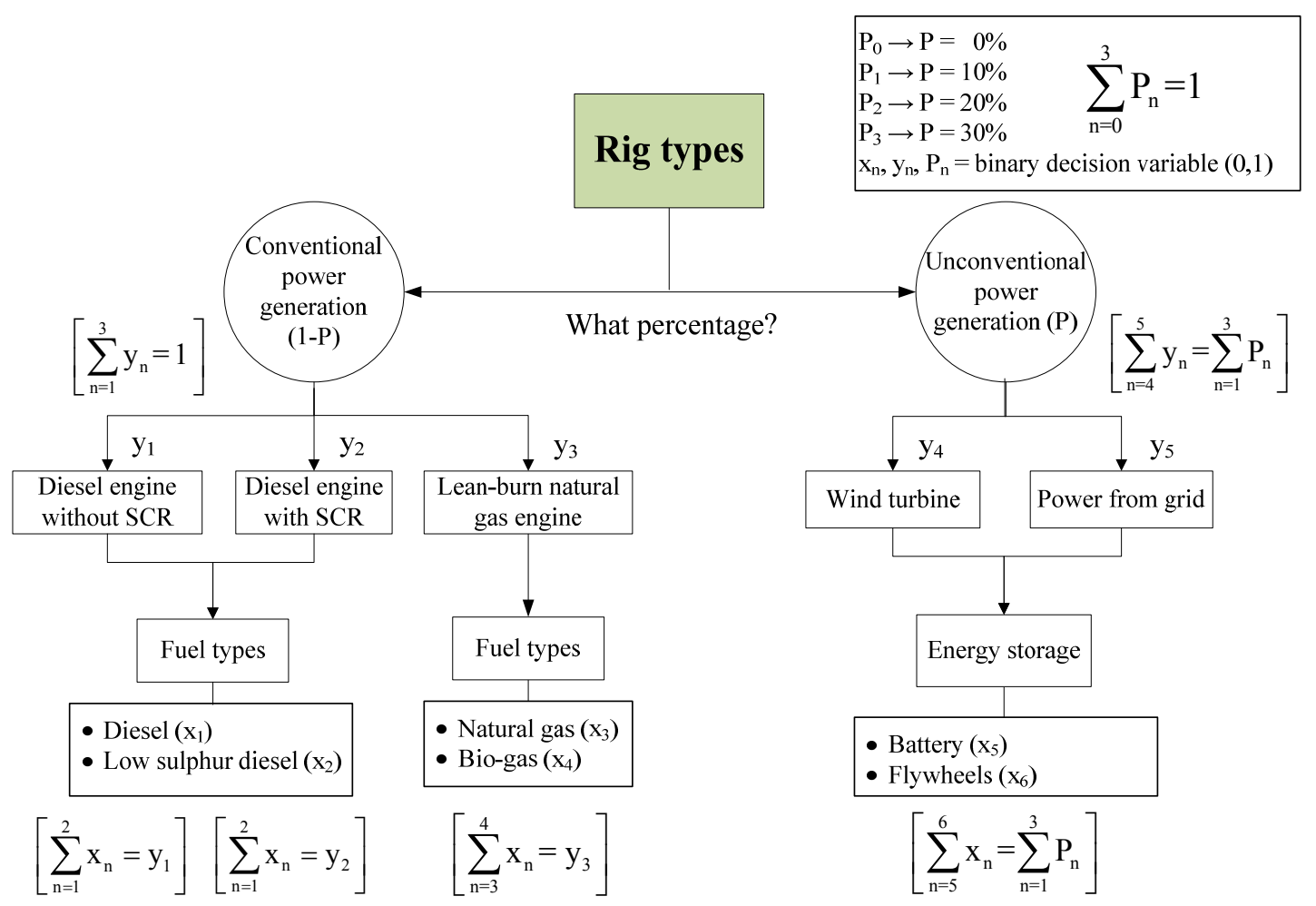

Figure 6-4. Selection procedure for the "Rig" subsystem of the case study

\begin{tabular}{|c|c|c|c|c|c|c|c|}
\hline $\begin{array}{c}\text { Sub- } \\
\text { sets }\end{array}$ & Technologies & Buy(\$) & $\begin{array}{c}\text { Resale } \\
\text { Value (\$) }\end{array}$ & $\begin{array}{c}\text { Rent /day } \\
\text { (\$) }\end{array}$ & $\begin{array}{c}\text { Daily } \\
\text { Rate (\$) }\end{array}$ & $\begin{array}{c}\text { Total cost } \\
\text { (\$) }\end{array}$ & $\begin{array}{c}\text { Ecological } \\
\text { Footprint } \\
\text { (Acres) }\end{array}$ \\
\hline 1 & \multicolumn{2}{|c|}{ Low sulphur diesel truck w/tier III engine, w/noise suppressor } & & & & & \\
\hline \multirow{3}{*}{2} & Gravel roads & $\$ 198,396$ & $\$ 0$ & & $\$ 19,840$ & $\$ 198,396$ & 3.030 \\
\hline & DURA-BASE from Composite Mat (buy) & $\$ 2,721,840$ & $\$ 2,177,472$ & & $\$ 54,437$ & $\$ 544,368$ & 1.515 \\
\hline & DURA-BASE from Composite Mat (rent) & & & & & $\$ 147,840$ & 1.515 \\
\hline \multirow{4}{*}{3} & Gravel pad & $\$ 135,270$ & $\$ 0$ & & $\$ 13,527$ & $\$ 135,270$ & 2.066 \\
\hline & DURA-BASE from Composite Mat (buy) & $\$ 1,855,800$ & $\$ 1,484,640$ & & $\$ 37,116$ & $\$ 371,160$ & 1.033 \\
\hline & DURA-BASE from Composite Mat (rent) & & & & & $\$ 100,800$ & 1.033 \\
\hline & Aluminum modules + driven piles & $\$ 1,131,303$ & $\$ 905,042$ & & $\$ 22,626$ & $\$ 226,261$ & 0.005 \\
\hline 4 & LOC250 (CWD) & & & $\$ 15,000$ & $\$ 15,000$ & $\$ 173,800$ & \\
\hline 5 & \multicolumn{2}{|l|}{ Lean-burn natural gas engines w/noise suppressor } & & $\$ 5,472$ & $\$ 5,472$ & $\$ 54,720$ & \\
\hline 6 & Natural gas & & & $\$ 2,100$ & $\$ 2,100$ & $\$ 19,950$ & \\
\hline 7 & Electric power from grid & & & $\$ 1,152$ & $\$ 1,152$ & $\$ 11,520$ & 0.000 \\
\hline 8 & Flywheels & $\$ 450,000$ & $\$ 360,000$ & & $\$ 9,000$ & $\$ 90,000$ & 0.000 \\
\hline 9 & Managed pressure drilling $w /$ noise suppressor & & & $\$ 21,500$ & $\$ 21,500$ & $\$ 193,500$ & \\
\hline 10 & Water-based muds & & & & & $\$ 47,940$ & \\
\hline \multirow{2}{*}{11} & Lined reserve pit + solid control equip.* & & & $\$ 2,000$ & $\$ 2,000$ & $\$ 18,000$ & 0.037 \\
\hline & Closed loop + containers + solid control equip. $*$ & & & $\$ 3,000$ & $\$ 3,000$ & $\$ 27,000$ & 0.000 \\
\hline
\end{tabular}

Figure 6-5. An example of input scores of the case study 
Once each technology is evaluated with respect to the nine attributes (i.e., $\mathrm{x}_{1}$ through $\mathrm{x}_{9}$ ), for each attribute, the overall attribute score of a system is calculated by adding the technology scores of the system or selecting the minimum technology score of the system. In order to calculate the overall score of a system on the $\mathrm{i}^{\text {th }}$ attribute $\left(\mathrm{X}_{\mathrm{i}}\right)$, Eq. (5-1), Eq. (5-2), and Eq. (5-3) should be considered.

As described in Section 5.6, A utility function is a relationship between the dimensional attribute score (e.g., \$, acres, and grades) and a non-dimensional number (between 0 and 1) that captures decision-maker preferences. Once the overall attribute score for each attribute of a system is calculated with respect to the nine attributes (i.e., $\mathrm{x}_{1}$ through $\mathrm{x}_{9}$ ), for each attribute (i) and in order to homogenize the scores, a utility function $\left(\mathrm{u}_{\mathrm{i}}\right)$ needs to be developed to convert the overall dimensional score of a system into a non-dimensional utility value (between 0 and 1 ) of the system. The proportional scoring approach given in Eq. (5-4) is mainly used in this case study to develop singleattribute utility functions except the noise attribute utility function.

According to Occupational Safety \& health Administration (OSHA), the employer shall administer a continuing, effective hearing conservation program if employee noise exposures equal or exceed an 8-hour time-weighted average sound level (TWA) of 85 decibels. In this case study, therefore, it is assumed that if TWA of a technology does not exceed 85 decibels, the noise utility score of the technology would be closed to one while the noise utility score of the technology would be rapidly down to zero if TWA of the technology exceeds 85 decibels. There are five noise making subsets $(2,3,4,5,9)$ in a system and thus it is considered that a utility value of the noise attribute $\left(\mathrm{x}_{5}\right)$ would be closed to one until a combined TWA does not exceed $425(5 \times$ 85) for a system. As a combined TWA exceeds 425, the utility value of the system rapidly goes down to zero. In order to satisfy these conditions, the noise attribute utility function is developed by the author as follows:

$$
\mathrm{u}_{5}\left(\mathrm{X}_{5}\right)=a+b \times c^{X_{5}}
$$

where $\mathrm{X}_{5}$ is the noise attribute score, TWA in decibels, of a system and $\mathrm{u}_{5}$ is the noise attribute utility value of the system. The constants (a, b, and c) are 1.02261, -8.5478E-07, 
and 1.028271, respectively. Figure 6-6 shows the utility function curves used in this case study.

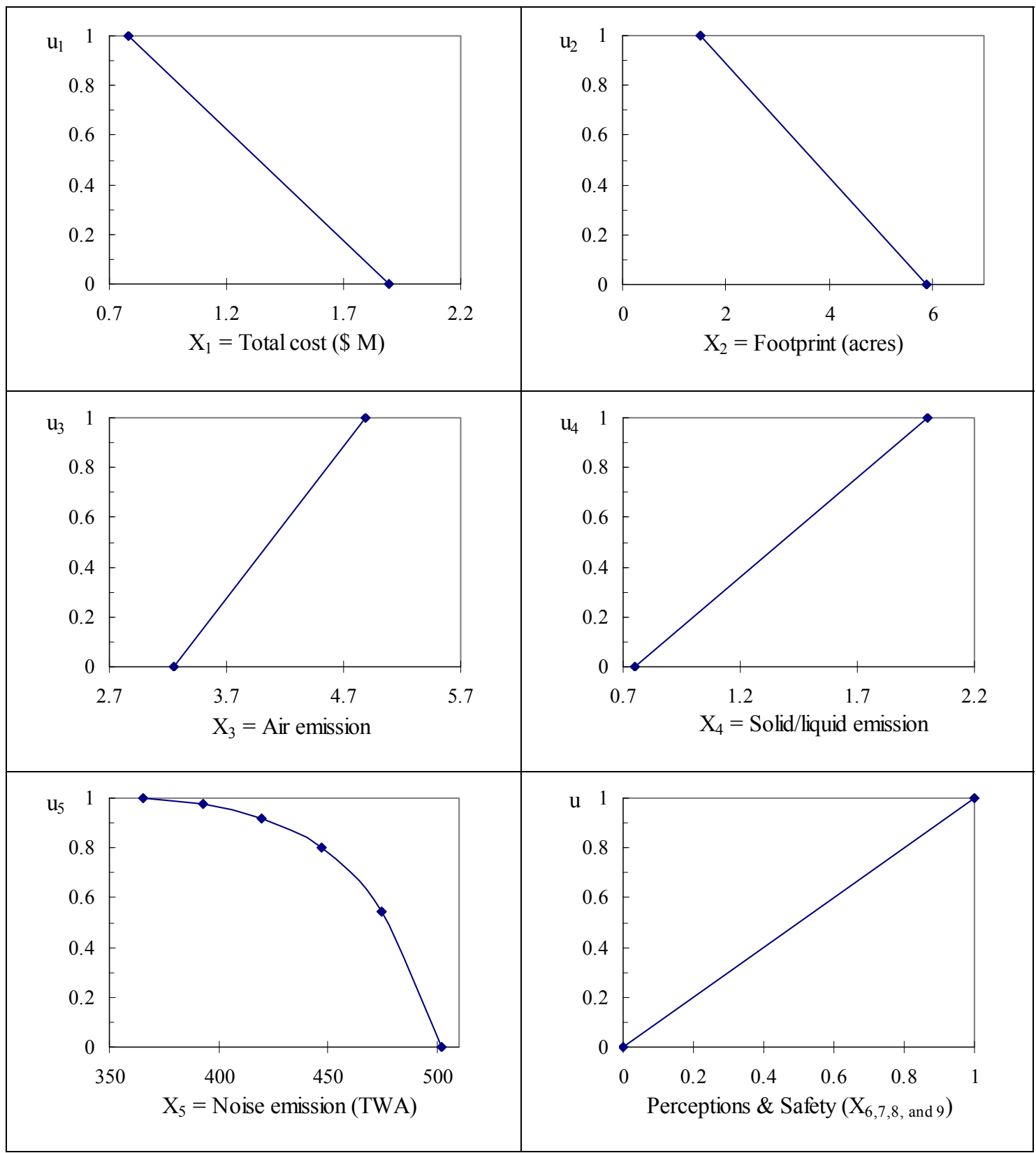

Figure 6-6. The single-attribute utility function curves

Once each single-attribute utility value is calculated, these individual utility values are combined into an overall utility value of a system described in Section 5.7. 
The base case weight factors are decided by an EFD expert in this case study as shown in Table 6-2. Since it is assumed that there is no interaction between each attribute, all of the weights are positive and they must sum to one. Figure 6-7 through Figure 6-9 show the overall utility values of the pre-specified systems described in Table 6-1 with the weighting factors given in Table 6-2.

Table 6-2. The base case weight factor for the each attribute

\begin{tabular}{|l|c|}
\hline \multicolumn{1}{|c|}{ Attributes } & Weights \\
\hline Total cost $\left(\mathrm{x}_{1}\right)$ & 0.40 \\
\hline Footprint $\left(\mathrm{x}_{2}\right)$ & 0.20 \\
\hline Air emission $\left(\mathrm{x}_{3}\right)$ & $0.20 / 3$ \\
\hline Solid/ liquid emission $\left(\mathrm{x}_{4}\right)$ & $0.20 / 3$ \\
\hline Noise emission $\left(\mathrm{x}_{5}\right)$ & $0.20 / 3$ \\
\hline Government perception $\left(\mathrm{x}_{6}\right)$ & 0.05 \\
\hline Industry perception $\left(\mathrm{x}_{7}\right)$ & 0.05 \\
\hline Public perception $\left(\mathrm{x}_{8}\right)$ & 0.05 \\
\hline Safety $\left(\mathrm{x}_{9}\right)$ & 0.05 \\
\hline
\end{tabular}

\begin{tabular}{|c|c|c|c|c|c|c|c|c|c|}
\hline & \multicolumn{9}{|c|}{ Weights $(\Sigma=100 \% \quad \therefore$ O.K! $)$} \\
\hline & $40 \%$ & $20 \%$ & $6.667 \%$ & $6.667 \%$ & $6.667 \%$ & $5 \%$ & $5 \%$ & $5 \%$ & $5 \%$ \\
\hline \multirow{2}{*}{$\begin{array}{l}\text { Selected Technologies } \\
\text { in Each Subset }\end{array}$} & \multirow{2}{*}{$\begin{array}{c}\text { Total Cost } \\
(\$)\end{array}$} & \multirow{2}{*}{$\begin{array}{c}\text { Ecological } \\
\text { Footprint } \\
\text { (Acres) }\end{array}$} & \multicolumn{3}{|c|}{ Emissions } & \multicolumn{3}{|c|}{ Perceptions } & \multirow{2}{*}{$\begin{array}{l}\text { Safety } \\
\text { Value }\end{array}$} \\
\hline & & & Air & $\begin{array}{l}\text { Solid\& } \\
\text { Liquid }\end{array}$ & $\begin{array}{l}\text { Noise } \\
\text { (TWA) }\end{array}$ & Gov. & Ind. & Public & \\
\hline (1) Transportation: Coventional diesel truck & & & & & & 0.250 & 1.000 & 0.250 & 0.750 \\
\hline (2) Road construction: Gravel roads & $\$ 148,500$ & 3.030 & 0.566 & & 98.562 & 0.250 & 1.000 & 0.250 & 0.500 \\
\hline (3) Site preparation: Gravel pad & $\$ 137,813$ & 2.812 & 0.598 & & 98.019 & 0.250 & 1.000 & 0.250 & 0.500 \\
\hline (4) Rig type: Traditional older vintage rig & $\$ 220,000$ & & 0.973 & & 78.630 & 0.500 & 1.000 & 0.500 & 0.500 \\
\hline (5) Rig power (Conventional): Internal combustion engine & $\$ 80,000$ & & 0.118 & & 110.073 & 0.500 & 1.000 & 0.500 & 0.750 \\
\hline (6) Fuel type: Conventional diesel & $\$ 94,080$ & & & & & 0.500 & 1.000 & 0.500 & 0.500 \\
\hline (7) Rig power (Unconventional): N/A (0 \%) & $\$ 0$ & 0.000 & 1.000 & & 0.000 & 0.250 & 1.000 & 0.250 & 1.000 \\
\hline (8) Energy storage: N/A & $\$ 0$ & 0.000 & & & & 0.250 & 1.000 & 0.250 & 1.000 \\
\hline (9) Drilling tech.: Conventional overbalanced drilling & $\$ 204,000$ & & & & 116.700 & 1.000 & 0.500 & 0.500 & 0.500 \\
\hline (10) Fluid type: Water-based muds & $\$ 47,940$ & & & & & 1.000 & 1.000 & 1.000 & 1.000 \\
\hline (11) Waste mgmt.: Lined reserve pit + solid control equip.* & $\$ 24,000$ & 0.037 & & 0.500 & & 0.750 & 0.750 & 0.750 & 0.500 \\
\hline (12) Cuttings mgmt.: Cuttings injection & $\$ 60,000$ & & & 1.000 & & 1.000 & 0.500 & 1.000 & 0.750 \\
\hline \multicolumn{10}{|l|}{ (13) Noise reduction: N/A } \\
\hline Overall Attribute Scores ( $\Sigma$ or minimum value) & $\$ 1,016,333$ & 5.879 & 3.254 & 1.500 & 501.985 & 0.250 & 0.500 & 0.250 & 0.500 \\
\hline Single Attribute Utility Values & 0.788 & 0.000 & 0.000 & 0.600 & 0.000 & 0.250 & 0.500 & 0.250 & 0.500 \\
\hline$\therefore$ Multi-Attribute Utility Value $=$ & $\underline{0.430}$ & & & & & & & & \\
\hline
\end{tabular}

Figure 6-7. Overall utility score of the conventional drilling system 


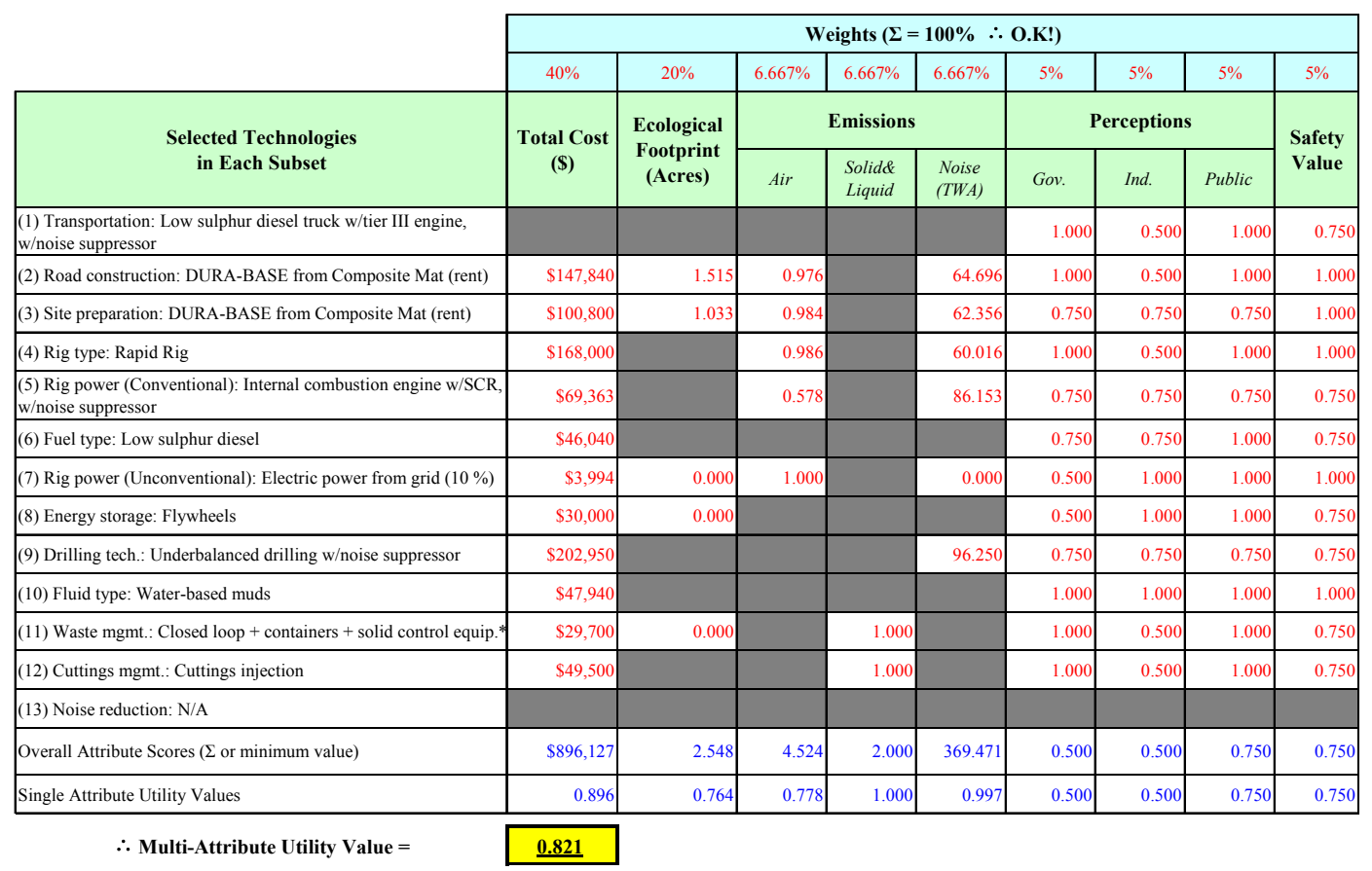

Figure 6-8. Overall utility score of the moderately improved drilling system

\begin{tabular}{|c|c|c|c|c|c|c|c|c|c|}
\hline \multirow{4}{*}{$\begin{array}{l}\text { Selected Technologies } \\
\text { in Each Subset }\end{array}$} & \multicolumn{9}{|c|}{ Weights $(\Sigma=100 \% \quad \therefore$ O.K!) } \\
\hline & \multirow{3}{*}{\begin{tabular}{|c|}
$40 \%$ \\
$\begin{array}{c}\text { Total Cost } \\
\text { (\$) }\end{array}$ \\
\end{tabular}} & \multirow{3}{*}{\begin{tabular}{c|}
$20 \%$ \\
$\begin{array}{c}\text { Ecological } \\
\text { Footprint } \\
\text { (Acres) }\end{array}$
\end{tabular}} & $6.667 \%$ & $6.667 \%$ & $6.667 \%$ & $5 \%$ & $5 \%$ & $5 \%$ & $5 \%$ \\
\hline & & & \multicolumn{3}{|c|}{ Emissions } & \multicolumn{3}{|c|}{ Perceptions } & \multirow{2}{*}{$\begin{array}{c}\text { Safety } \\
\text { Value }\end{array}$} \\
\hline & & & Air & $\begin{array}{l}\text { Solid\& } \\
\text { Liquid }\end{array}$ & $\begin{array}{l}\text { Noise } \\
\text { (TWA) }\end{array}$ & Gov. & Ind. & Public & \\
\hline $\begin{array}{l}\text { (1) Transportation: Low sulphur diesel truck w/tier III engine, } \\
\text { w/noise suppressor }\end{array}$ & & & & & & 1.000 & 0.500 & 1.000 & 0.750 \\
\hline (2) Road construction: DURA-BASE from Composite Mat (rent) & $\$ 147,840$ & 1.515 & 0.976 & & 64.696 & 1.000 & 0.500 & 1.000 & 1.000 \\
\hline (3) Site preparation: Aluminum modules + driven piles & $\$ 226,261$ & 0.005 & 0.989 & & 76.265 & 1.000 & 0.500 & 1.000 & 0.500 \\
\hline (4) Rig type: LOC250 (CWD) & $\$ 173,800$ & & 0.985 & & 60.366 & 1.000 & 0.500 & 1.000 & 1.000 \\
\hline $\begin{array}{l}\text { (5) Rig power (Conventional): Lean-burn natural gas engines } \\
\text { w/noise suppressor }\end{array}$ & $\$ 54,720$ & & 0.936 & & 83.742 & 1.000 & 0.500 & 1.000 & 0.750 \\
\hline (6) Fuel type: Natural gas & $\$ 19,950$ & & & & & 1.000 & 0.500 & 1.000 & 0.750 \\
\hline (7) Rig power (Unconventional): Electric power from grid (30 \%) & $\$ 11,520$ & 0.000 & 1.000 & & 0.000 & 0.500 & 1.000 & 1.000 & 1.000 \\
\hline (8) Energy storage: Flywheels & $\$ 90,000$ & 0.000 & & & & 0.500 & 1.000 & 1.000 & 0.750 \\
\hline (9) Drilling tech.: Managed pressure drilling w/noise suppressor & $\$ 193,500$ & & & & 94.100 & 0.750 & 0.750 & 1.000 & 1.000 \\
\hline (10) Fluid type: Water-based muds & $\$ 47,940$ & & & & & 1.000 & 1.000 & 1.000 & 1.000 \\
\hline (11) Waste mgmt.: Closed loop + containers + solid control equip." & $\$ 27,000$ & 0.000 & & 1.000 & & 1.000 & 0.500 & 1.000 & 0.750 \\
\hline (12) Cuttings mgmt:: Chemical fixation and solidification (CFS) & $\$ 61,710$ & & & 0.250 & & 0.750 & 0.750 & 1.000 & 0.500 \\
\hline \multicolumn{10}{|l|}{ (13) Noise reduction: N/A } \\
\hline Overall Attribute Scores ( $\Sigma$ or minimum value) & $\$ 1,054,240$ & 1.520 & 4.886 & 1.250 & 379.169 & 0.500 & 0.500 & 1.000 & 0.500 \\
\hline Single Attribute Utility Values & 0.754 & 1.000 & 1.000 & 0.400 & 0.989 & 0.500 & 0.500 & 1.000 & 0.500 \\
\hline$\therefore$ Multi-Attribute Utility Value $=$ & $\underline{0.786}$ & & & & & & & & \\
\hline
\end{tabular}

Figure 6-9. Overall utility score of the EFD system in five years 
In this case study, since an exhaustive search optimization is a simple, practical, and very robust method given the speed of modern computers (Cover et al. 2007), it is used to evaluate all possible systems and to find the 'best' available system that should be particularly attractive for Green Lake drilling site. Larger problems would likely require more advanced optimization methods. Figure 6-10 briefly illustrates the total possible number of systems used in this case study. Once all possible systems have been evaluated, the system with the highest overall utility score is the best system with given weighting factors. Figure 6-11 shows the overall utility value of the best system with the weighting factors given in Table 6-2.

1. When "Diesel engine" is selected as a conventional power generation,

\begin{tabular}{|c|c|c|c|c|c|}
\hline \multirow{2}{*}{ Subsets } & \multicolumn{4}{|c|}{ Subsystems } & \multirow{2}{*}{$\prod$} \\
\hline & 1. Access & 2. Drill Site & 3. Rig & 4. Drilling & \\
\hline (1) & 2 & 4 & 3 & 3 & 72 \\
\hline (2) & 3 & & $2 *$ & 1 & 6 \\
\hline (3) & & & 2 & 2 & 4 \\
\hline (4) & & & 1 & 2 & 2 \\
\hline (5) & & & 1 & 1 & 1 \\
\hline & & & & $\Pi$ & 3,456 \\
\hline
\end{tabular}

2. When "Natural gas engine" is selected as a conventional power generation,

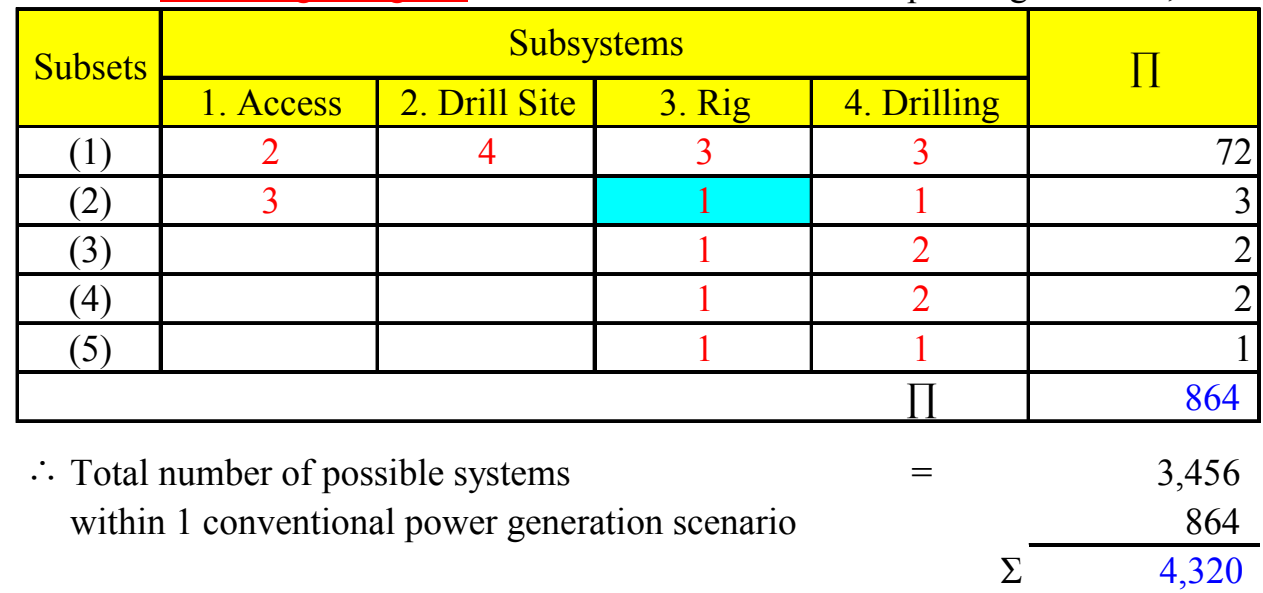

4 different portions of unconventional power usage $(0,10,20,30 \%)$ were consider
$\therefore$ Total number of iterations $=$
4
$\times 4320$
$=17280$

*: 2 types of diesel engine

Figure 6-10. Total number of possible systems used in this cast study 


\begin{tabular}{|c|c|c|c|c|c|c|c|c|c|}
\hline & \multicolumn{9}{|c|}{ Weights $(\Sigma=100 \% \quad \therefore$ O.K!) } \\
\hline & $40 \%$ & $20 \%$ & $6.667 \%$ & $6.667 \%$ & $6.667 \%$ & $5 \%$ & $5 \%$ & $5 \%$ & $5 \%$ \\
\hline \multirow{2}{*}{$\begin{array}{l}\text { Selected Technologies } \\
\text { in Each Subset }\end{array}$} & \multirow{2}{*}{$\begin{array}{c}\text { Total Cost } \\
(\$)\end{array}$} & \multirow{2}{*}{$\begin{array}{c}\text { Ecological } \\
\text { Footprint } \\
\text { (Acres) }\end{array}$} & \multicolumn{3}{|c|}{ Emissions } & \multicolumn{3}{|c|}{ Perceptions } & \multirow{2}{*}{$\begin{array}{l}\text { Safety } \\
\text { Value }\end{array}$} \\
\hline & & & Air & $\begin{array}{l}\text { Solid\& } \\
\text { Liquid }\end{array}$ & $\begin{array}{l}\text { Noise } \\
\text { (TWA) }\end{array}$ & Gov. & Ind. & Public & \\
\hline $\begin{array}{l}\text { (1) Transportation: Low sulphur diesel truck w/tier III engine, } \\
\text { w/noise suppressor }\end{array}$ & & & & & & 1.000 & 0.500 & 1.000 & 0.750 \\
\hline (2) Road construction: DURA-BASE from Composite Mat (rent) & $\$ 147,840$ & 1.515 & 0.976 & & 64.696 & 1.000 & 0.500 & 1.000 & 1.000 \\
\hline (3) Site preparation: DURA-BASE from Composite Mat (rent) & $\$ 100,800$ & 1.033 & 0.984 & & 62.356 & 0.750 & 0.750 & 0.750 & 1.000 \\
\hline (4) Rig type: LOC250 (CWD) & $\$ 173,800$ & & 0.985 & & 60.366 & 1.000 & 0.500 & 1.000 & 1.000 \\
\hline $\begin{array}{l}\text { (5) Rig power (Conventional): Lean-burn natural gas engines } \\
\text { w/noise suppressor }\end{array}$ & $\$ 70,354$ & & 0.918 & & 85.603 & 1.000 & 0.500 & 1.000 & 0.750 \\
\hline (6) Fuel type: Natural gas & $\$ 25,650$ & & & & & 1.000 & 0.500 & 1.000 & 0.750 \\
\hline (7) Rig power (Unconventional): Electric power from grid (10\%) & $\$ 3,840$ & 0.000 & 1.000 & & 0.000 & 0.500 & 1.000 & 1.000 & 1.000 \\
\hline (8) Energy storage: Flywheels & $\$ 30,000$ & 0.000 & & & & 0.500 & 1.000 & 1.000 & 0.750 \\
\hline (9) Drilling tech.: Underbalanced drilling w/noise suppressor & $\$ 184,500$ & & & & 95.700 & 0.750 & 0.750 & 0.750 & 0.750 \\
\hline (10) Fluid type: Water-based muds & $\$ 47,940$ & & & & & 1.000 & 1.000 & 1.000 & 1.000 \\
\hline (11) Waste mgmt.: Closed loop + containers + solid control equip." & $\$ 27,000$ & 0.000 & & 1.000 & & 1.000 & 0.500 & 1.000 & 0.750 \\
\hline (12) Cuttings mgmt.: Cuttings injection & $\$ 45,000$ & & & 1.000 & & 1.000 & 0.500 & 1.000 & 0.750 \\
\hline \multicolumn{10}{|l|}{ (13) Noise reduction: N/A } \\
\hline Overall Attribute Scores ( $\Sigma$ or minimum value) & $\$ 856,724$ & 2.548 & 4.863 & 2.000 & 368.721 & 0.500 & 0.500 & 0.750 & 0.750 \\
\hline Single Attribute Utility Values & 0.931 & 0.764 & 0.986 & 1.000 & 0.998 & 0.500 & 0.500 & 0.750 & 0.750 \\
\hline$\therefore$ Multi-Attribute Utility Value $=$ & 849 & & & & & & & & \\
\hline
\end{tabular}

Figure 6-11. Overall utility score of the best system

Figure 6-12 shows the comparison of the single-attribute utility values of the prespecified systems given in Table 6-1 and the best system with the weighting factors given in Table 6-2. It is indicated that the overall utility score of the System 2, "Moderately improved drilling system", is greater than the utility score of the system 3, "EFD system in five years."

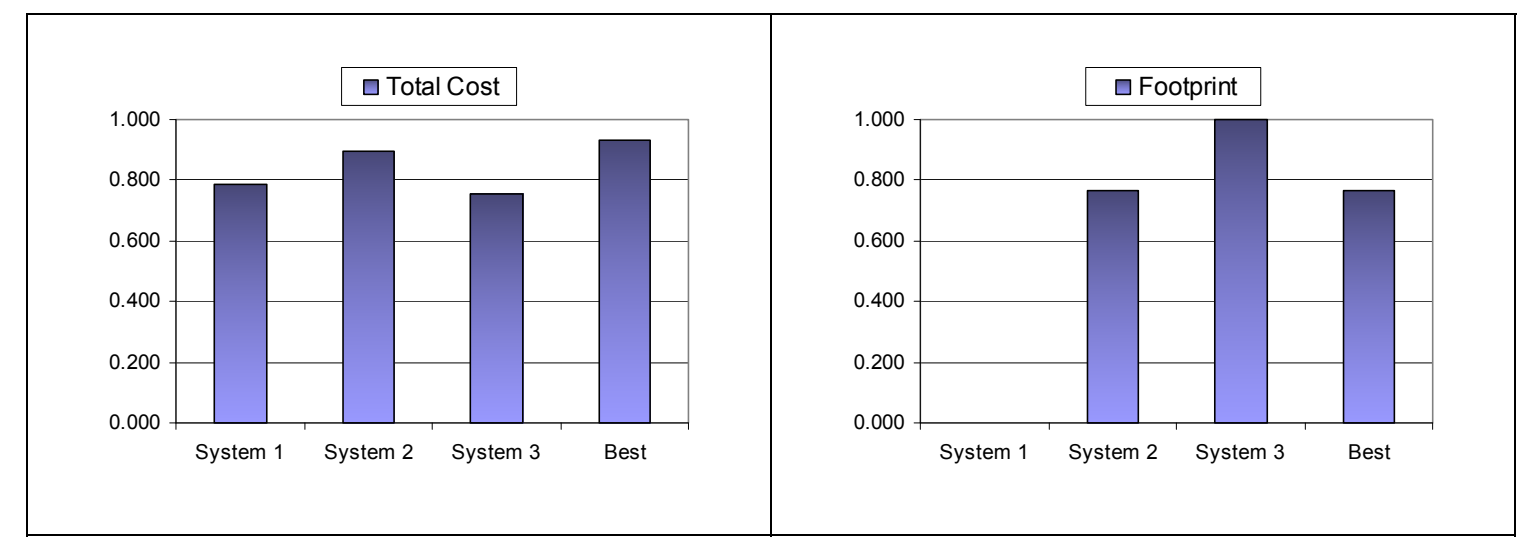

Figure 6-12. Comparison of single-attribute utility scores and overall utility scores 


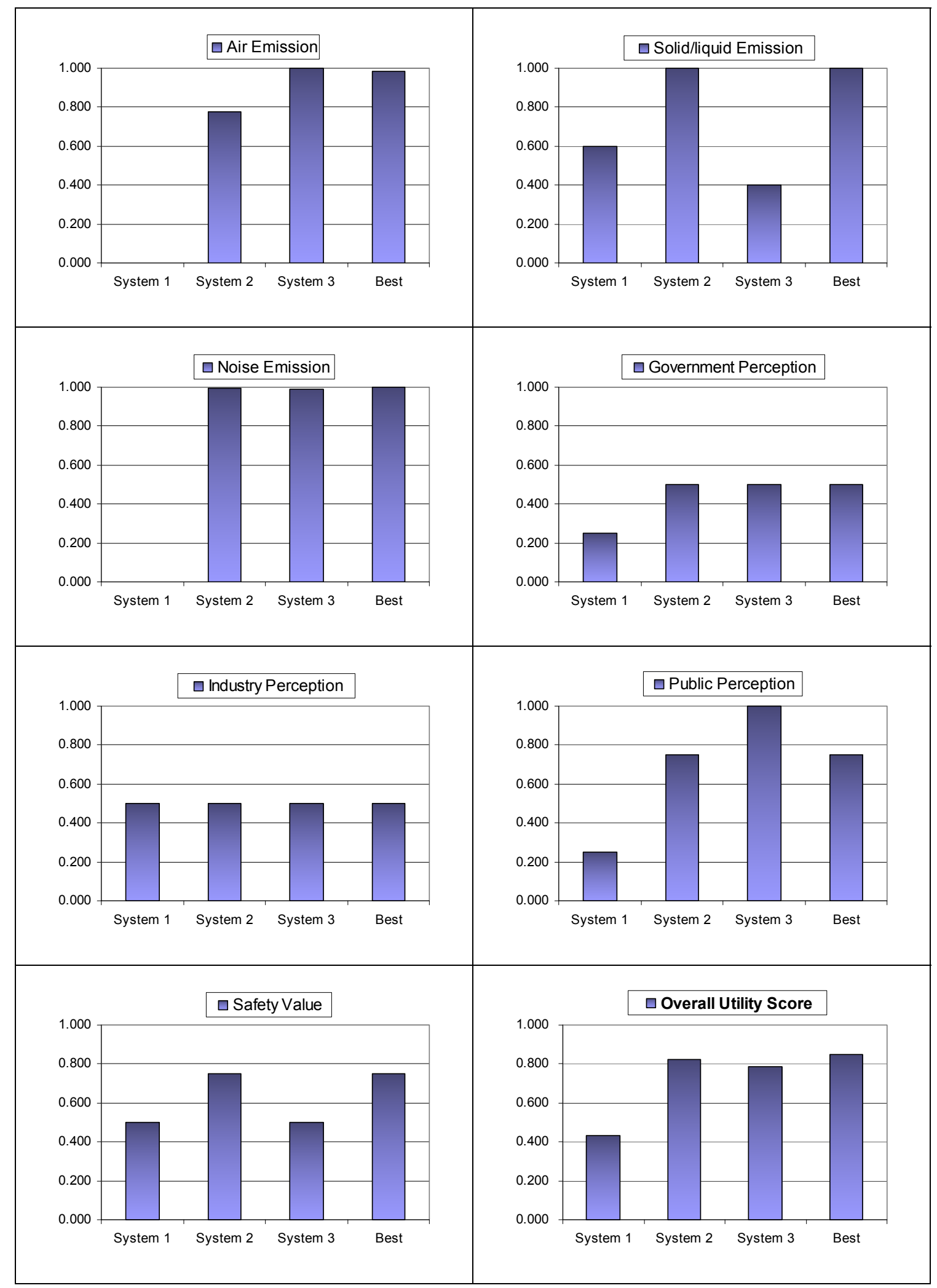

Figure 6-12. Continued 


\subsection{Conduct a Sensitivity Analysis}

After the optimization scheme has given the 'best' system, a sensitivity analysis can be conducted to examine the impact of possible changes in the attribute scores, weight factors, and utility functions on the best system. For example, the weights assigned to cost attribute could be changed from the initially assigned value of 0.40 given in Table 6-2. Since the weighting factors must sum to one in this case study, the weights assigned to other attributes are known once a weight assigned to cost attribute is decided. Conducting a sensitivity analysis for the technology selection process is an importance step because it can give an idea the range of weights over which certain systems should be selected for a specific site (Guikema and Milke 1999). This step contains two sections. One is a sensitivity analysis for weighting factors of each attribute and the other one is a sensitivity analysis for uncertainty of overall attribute scores.

\subsubsection{Sensitivity Analysis for Weighting Factors of Each Attribute}

In order to demonstrate how a sensitivity analysis can be conducted, four different weight scenarios are defined by an EFD expert as shown in Table 6-3, the optimization routine is run for each weight combination, and then the results are compared as shown in Table 6-4. Each of those weight combinations represents a different point of view for the EFD technology selection problem. In this step, the change in the overall utility score of the optimal system is not a good sensitivity measure because the overall utility score directly depends on the input parameters being used and there are also many uncertainties in those input values. Instead, it is suggested to look at the changes in the technologies selected for the optimal system because this is the decision that is the most interest to the decision-makers.

Table 6-3. Weight combinations used in the sensitivity analysis

\begin{tabular}{|c|c|c|c|c|c|c|c|c|c|c|}
\hline Weight & Cost & Footprint & \multicolumn{3}{|c|}{ Emissions $\left(\mathrm{W}_{3}\right)$} & \multicolumn{3}{c|}{ Perception $\left(\mathrm{W}_{4}\right)$} & Safety & \multirow{2}{*}{ Note } \\
\cline { 4 - 11 } & $\left(\mathrm{W}_{1}\right)$ & $\left(\mathrm{W}_{2}\right)$ & Air & $\mathrm{S} / \mathrm{L}$ & Noise & Gov. & Ind. & Public & $\left(\mathrm{W}_{5}\right)$ & \\
\hline 1 & 0.60 & 0.25 & $0.05 / 3$ & $0.05 / 3$ & $0.05 / 3$ & $0.05 / 3$ & $0.05 / 3$ & $0.05 / 3$ & 0.05 & Conventional \\
\hline 2 & 0.40 & 0.20 & $0.20 / 3$ & $0.20 / 3$ & $0.20 / 3$ & 0.05 & 0.05 & 0.05 & 0.05 & Base case \\
\hline 3 & 0.27 & 0.25 & 0.08 & 0.08 & 0.08 & 0.05 & 0.05 & 0.07 & 0.07 & EFD \\
\hline 4 & 0.12 & 0.30 & 0.10 & 0.10 & 0.10 & 0.05 & 0.05 & 0.09 & 0.09 & More EFD \\
\hline
\end{tabular}


Table 6-4. Results of the sensitivity analysis

\begin{tabular}{|c|c|c|c|}
\hline Subsets & For weight no. 1 & For weight no. 2 & $\begin{array}{l}\text { For weight no. } 3 \\
\text { and no. } 4\end{array}$ \\
\hline (1) Transportation & $\begin{array}{l}\text { Conventional diesel } \\
\text { truck }\end{array}$ & $\begin{array}{l}\text { Low sulphur diesel } \\
\text { truck w/tier III } \\
\text { engine, w/noise } \\
\text { suppressor }\end{array}$ & $\begin{array}{l}\text { Low sulphur diesel } \\
\text { truck w/tier III } \\
\text { engine, w/noise } \\
\text { suppressor }\end{array}$ \\
\hline $\begin{array}{l}\text { (2) Road } \\
\text { construction }\end{array}$ & $\begin{array}{l}\text { DURA-BASE from } \\
\text { Composite Mat (rent) }\end{array}$ & $\begin{array}{l}\text { DURA-BASE from } \\
\text { Composite Mat (rent) }\end{array}$ & $\begin{array}{l}\text { DURA-BASE from } \\
\text { Composite Mat (rent) }\end{array}$ \\
\hline (3) Site preparation & $\begin{array}{l}\text { DURA-BASE from } \\
\text { Composite Mat (rent) }\end{array}$ & $\begin{array}{l}\text { DURA-BASE from } \\
\text { Composite Mat (rent) }\end{array}$ & $\begin{array}{l}\text { Aluminum modules }+ \\
\text { driven piles (elevated } \\
\text { platform) }\end{array}$ \\
\hline (4) Rig type & LOC250 (CWD) & LOC250 (CWD) & LOC250 (CWD) \\
\hline $\begin{array}{l}\text { (5) Conventional } \\
\text { rig power } \\
\text { engine }\end{array}$ & $\begin{array}{l}\text { Lean-burn natural gas } \\
\text { engines w/noise } \\
\text { suppressor }\end{array}$ & $\begin{array}{l}\text { Lean-burn natural gas } \\
\text { engines } \mathrm{w} / \text { noise } \\
\text { suppressor }\end{array}$ & $\begin{array}{l}\text { Lean-burn natural gas } \\
\text { engines w/noise } \\
\text { suppressor }\end{array}$ \\
\hline (6) Fuel type & Natural gas & Natural gas & Natural gas \\
\hline $\begin{array}{l}\text { (7) Unconventional } \\
\text { rig power } \\
\text { generation }\end{array}$ & None & $\begin{array}{l}\text { Electric power from } \\
\text { grid }(10 \%)\end{array}$ & $\begin{array}{l}\text { Electric power from } \\
\text { grid }(10 \%)\end{array}$ \\
\hline (8) Energy storage & None & Flywheel & Flywheel \\
\hline $\begin{array}{l}\text { (9) Drilling } \\
\text { technology }\end{array}$ & $\begin{array}{l}\text { Underbalanced } \\
\text { drilling w/noise } \\
\text { suppressor }\end{array}$ & $\begin{array}{l}\text { Underbalanced } \\
\text { drilling w/noise } \\
\text { suppressor }\end{array}$ & $\begin{array}{l}\text { Managed pressure } \\
\text { drilling w/noise } \\
\text { suppressor }\end{array}$ \\
\hline (10) Fluid type & Water-based muds & Water-based muds & Water-based muds \\
\hline $\begin{array}{l}\text { (11) Drilling fluid } \\
\text { and waste } \\
\text { management }\end{array}$ & $\begin{array}{l}\text { Closed loop }+ \\
\text { containers }+ \text { solid } \\
\text { control equipment }\end{array}$ & $\begin{array}{l}\text { Closed loop }+ \\
\text { containers }+ \text { solid } \\
\text { control equipment }\end{array}$ & $\begin{array}{l}\text { Closed loop }+ \\
\text { containers }+ \text { solid } \\
\text { control equipment }\end{array}$ \\
\hline $\begin{array}{l}\text { (12) Cuttings } \\
\text { treatment }\end{array}$ & Cuttings injection & Cuttings injection & Cuttings injection \\
\hline $\begin{array}{l}\text { (13) } \begin{array}{l}\text { Noise } \\
\text { reduction }\end{array} \\
\end{array}$ & None & None & None \\
\hline
\end{tabular}

In order to generate the combinations of weights required to conduct this sensitivity analysis method, upper and lower bounds on the parameters need to be assessed. This can be done by asking to real project staffs or decision-makers. In this case study, the upper and lower bounds of each attribute weight are decided as shown in Table 6-5. 
Table 6-5. Range of the allowable weight factor for each attribute

\begin{tabular}{|c|c|c|c|c|c|c|c|c|c|}
\hline \multirow{2}{*}{ Weights } & Cost & Footprint & \multicolumn{3}{|c|}{ Emissions $\left(\mathrm{W}_{3}\right)$} & \multicolumn{3}{c|}{ Perception $\left(\mathrm{W}_{4}\right)$} & Safety \\
\cline { 4 - 8 } & $\left(\mathrm{W}_{1}\right)$ & $\left(\mathrm{W}_{2}\right)$ & Air & $\mathrm{S} / \mathrm{L}$ & Noise & Gov. & Ind. & Public & $\left(\mathrm{W}_{5}\right)$ \\
\hline Maximum & 1.00 & 1.00 & $1 / 3$ & $1 / 3$ & $1 / 3$ & $1 / 3$ & $1 / 3$ & $1 / 3$ & 1.00 \\
\hline Minimum & 0.00 & 0.00 & 0.00 & 0.00 & 0.00 & 0.00 & 0.00 & 0.00 & 0.00 \\
\hline
\end{tabular}

Based on the ranges given in Table 6-5, this study enumerates all possible weight combinations within these bounds that summed to one in increments of roughly 0.1 . Since the weights must sum to one, as one weight increases, others must decrease. In this case study, for example, as $\mathrm{w}_{1}$ increases, other weights (i.e., $\mathrm{w}_{2}, \mathrm{w}_{3}, \mathrm{w}_{4}$, and $\mathrm{w}_{5}$ ) decrease by the ratio of the weight combination shown in Table 6-3. For example, the weights assigned to cost attribute could be changed from the initially assigned value of 0.60 , 'Conventional case (weight no.1)', given in Table 6-2. Since the weighting factors must sum to one, the weights assigned to other attributes are known once a weight assigned to cost attribute is decided. It is noted that since the ratio of an assigned weight for each attribute is different from each weight combination, even if weights assigned to the same attribute are identical and increase by equal percentage for two different weight combinations, the weights assigned to other attributes decrease by the different ratio for the two weight combinations. The total number of weight combinations for further consideration is about 410 in this sensitivity analysis.

Deciding on the number of combinations of weights being used in a sensitivity analysis usually involves a trade-off between increased computational time for the analysis and the potential for increased modeling accuracy. This trade-off needs to be made on a case-specific basis (Guikema and Milke 2003). Once the combinations of input parameters are defined, the optimization routine is performed for each combination. This has the potential to consume significant time in the process, especially for problems where a large number of technologies are considered.

In this sensitivity analysis where weights are varied, twelve different drilling systems are selected as the optimal systems for at least one of the weight combinations being considered. Table 6-6 shows the proportion of each of twelve systems selected for this sensitivity analysis. The fact that SET 1, the most selected optimal solution, is 
selected for $42 \%$ of the weight combinations emphasizes the need for a sensitivity analysis.

Table 6-6. Proportion of the optimal systems for this case study

\begin{tabular}{|c|c|c|c|c|c|c|c|c|c|c|c|c|}
\hline & \multicolumn{10}{|c|}{ SET NUMBER } \\
\cline { 2 - 11 } & 1 & 2 & 3 & 4 & 5 & 6 & 7 & 8 & 9 & 10 & 11 & 12 \\
\hline $\begin{array}{c}\text { Proportion } \\
(\%)\end{array}$ & 41.6 & 35.0 & 9.1 & 4.7 & 2.0 & 1.5 & 1.5 & 1.5 & 1.5 & 0.7 & 0.5 & 0.5 \\
\hline
\end{tabular}

Throughout the sensitivity analysis conducted in this case study, six different drilling systems are suggested for Green Lake drilling site as shown in Figure 6-13. Figure 6-13 shows which technologies are selected for each suggested system.

\begin{tabular}{|c|c|}
\hline SET $1(41.6 \%)$ & SET $2(35 \%)$ \\
\hline $\begin{array}{l}\text { (1) Transportation: Low sulphur diesel truck w/tier III engine, } \\
\text { w/noise suppressor }\end{array}$ & $\begin{array}{l}\text { (1) Transportation: Low sulphur diesel truck w/tier III engine, } \\
\text { w/noise suppressor }\end{array}$ \\
\hline (2) Road construction: DURA-BASE from Composite Mat (rent) & (2) Road construction: DURA-BASE from Composite Mat (rent) \\
\hline (3) Site preparation: Aluminum modules + driven piles & (3) Site preparation: DURA-BASE from Composite Mat (rent) \\
\hline (4) Rig type: LOC250 (CWD) & (4) Rig type: LOC250 (CWD) \\
\hline $\begin{array}{l}\text { (5) Rig power (Conventional): Lean-burn natural gas engines } \\
\text { w/noise suppressor }\end{array}$ & $\begin{array}{c}\text { (5) Rig power (Conventional): Lean-burn natural gas engines } \\
\text { w/noise suppressor }\end{array}$ \\
\hline (6) Fuel type: Natural gas & (6) Fuel type: Natural gas \\
\hline (7) Rig power (Unconventional): Electric power from grid (10\%) & (7) Rig power (Unconventional): Electric power from grid (10\%) \\
\hline (8) Energy storage: Flywheels & (8) Energy storage: Flywheels \\
\hline (9) Drilling tech.: Managed pressure drilling w/noise suppressor & (9) Drilling tech.: Underbalanced drilling w/noise suppressor \\
\hline (10) Fluid type: Water-based muds & (10) Fluid type: Water-based muds \\
\hline (11) Waste mgmt.: Closed loop + containers + solid control equip.* & (11) Waste mgmt.: Closed loop + containers + solid control equip.* \\
\hline (12) Cuttings mgmt.: Cuttings injection & (12) Cuttings mgmt.: Cuttings injection \\
\hline (13) Noise reduction: N/A & (13) Noise reduction: N/A \\
\hline SET $3(9.1 \%)$ & SET $4(4.7 \%)$ \\
\hline (1) Transportation: Coventional diesel truck & $\begin{array}{l}\text { (1) Transportation: Low sulphur diesel truck w/tier III engine, } \\
\text { w/noise suppressor }\end{array}$ \\
\hline (2) Road construction: DURA-BASE & (2) Road construction: DURA-BASE from Composite Mat (rent) \\
\hline (3) Site preparation: DURA-BASE from Composite Mat (rent) & (3) Site preparation: Aluminum modules + driven piles \\
\hline (4) Rig type: LOC250 (CWD) & (4) Rig type: LOC250 (CWD) \\
\hline $\begin{array}{l}\text { (5) Rig power (Conventional): Lean-burn natural gas engines } \\
\text { w/noise suppressor }\end{array}$ & $\begin{array}{c}\text { (5) Rig power (Conventional): Lean-burn natural gas engines } \\
\text { w/noise suppressor }\end{array}$ \\
\hline (6) Fuel type: Natural gas & (6) Fuel type: Natural gas \\
\hline (7) Rig power (Unconventional): N/A (0\%) & (7) Rig power (Unconventional): Electric power from grid (30\%) \\
\hline (8) Energy storage: N/A & (8) Energy storage: Flywheels \\
\hline (9) Drilling tech.: Underbalanced drilling w/noise suppressor & (9) Drilling tech.: Managed pressure drilling w/noise su \\
\hline (10) Fluid type: Water-based muds & (10) Fluid type: Water-based muds \\
\hline (11) Waste mgmt.: Closed loop + containers + solid control equip.* & (11) Waste mgmt.: Closed loop + containers + solid control equip.* \\
\hline (12) Cuttings mgmt.: Cuttings injection & (12) Cuttings mgmt.: Cuttings injection \\
\hline (13) Noise reduction: N/A & (13) Noise reduction: N/A \\
\hline SET $5(2 \%)$ & SET $6(1.5 \%)$ \\
\hline (1) Transportation: Coventional diesel truck & (1) Transportation: Coventional diesel truck \\
\hline (2) Road construction: DURA-BASE from Composite Mat (rent) & (2) Road construction: DURA-BASE from Composite Mat (rent) \\
\hline (3) Site preparation: DURA-BASE from Composite Mat (rent) & (3) Site preparation: DURA-BASE from Composite Mat (rent) \\
\hline (4) Rig type: LOC250 (CWD) & (4) Rig type: LOC250 (CWD) \\
\hline (5) Rig power (Conventional): Internal combustion engine & (5) Rig power (Conventional): Internal combustion engine \\
\hline (6) Fuel type: Low sulphur diesel & (6) Fuel type: Conventional diesel \\
\hline (7) Rig power (Unconventional): N/A (0 \%) & (7) Rig power (Unconventional): N/A (0 \%) \\
\hline (8) Energy storage: N/A & (8) Energy storage: N/A \\
\hline (9) Drilling tech.: Underbalanced drilling w/noise suppressor & (9) Drilling tech.: Underbalanced drilling w/noise suppressor \\
\hline (10) Fluid type: Water-based muds & (10) Fluid type: Water-based muds \\
\hline (11) Waste mgmt.: Closed loop + containers + solid control equip.* & (11) Waste mgmt.: Lined reserve pit + solid control equip.* \\
\hline (12) Cuttings mgmt.: Cuttings injection & (12) Cuttings mgmt.: Cuttings injection \\
\hline (13) Noise reduction: N/A & (13) Noise reduction: N/A \\
\hline
\end{tabular}

Figure 6-13. Six systems suggested for Green Lake drilling site 
As can be seen in Figure 6-13, the results of six systems can indicate the potential for further simplification of a technology selection problem. In this case, the technologies selected for five subsets (i.e., (2), (4), (10), (12), and (13)) are always same in all suggested systems. Therefore, if sensitivity to weights is the only concern, the optimal decision would revolve around the technologies for only eight subsets of the original thirteen subsets.

Figure 6-14 shows the comparison of the single-attribute utility values of six suggested drilling systems given in Figure 6-13. Input values used in those suggested drilling systems can be found in APPENDIX B.

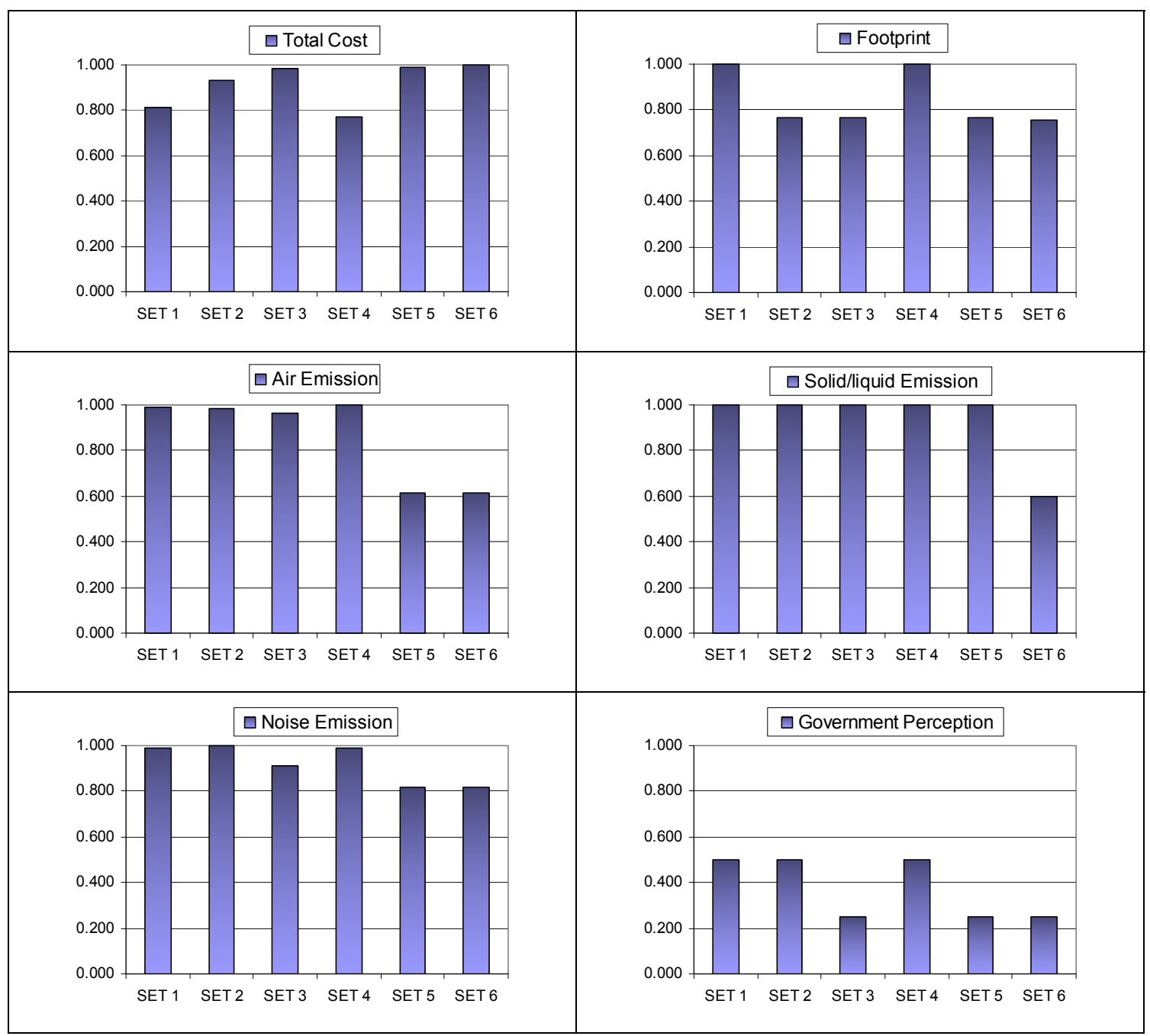

Figure 6-14. Comparison of the single-attribute utility scores 


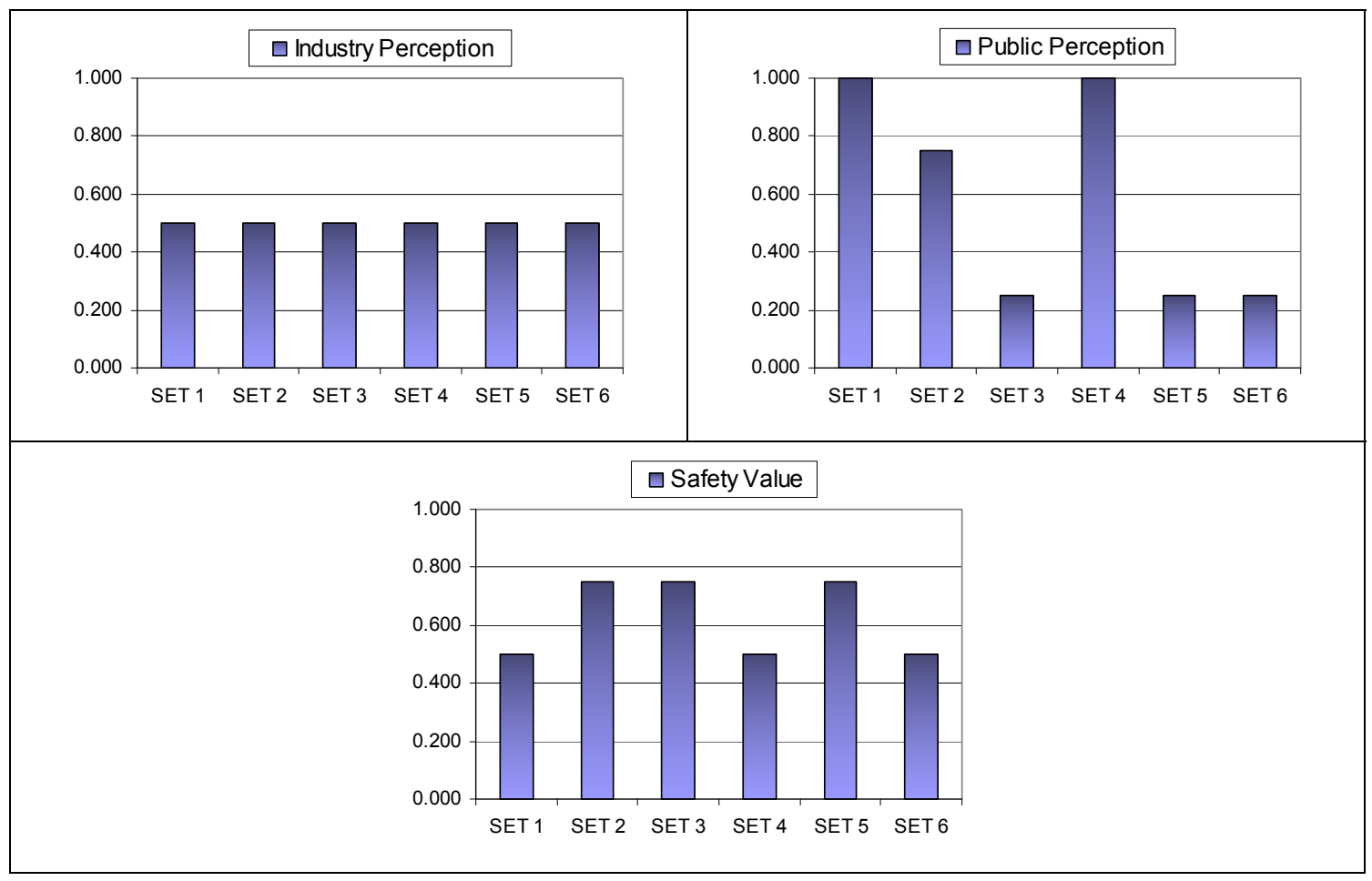

Figure 6-14. Continued

Figure 6-15 through Figure 6-19 show which system should be selected as weight 1 through weight $5\left(\mathrm{w}_{1} \sim \mathrm{w}_{5}\right)$ are varied respectively by the ratio of the base-case weight combination described in Table 6-3. Since the weights must sum to one, as one weight increases, others must decrease. In Figure 6-15, for example, as $\mathrm{w}_{1}$ increases, other weights (i.e., $\mathrm{w}_{2}$ through $\mathrm{w}_{5}$ ) decrease by the ratio of the base-case weight combination shown in Table 6-3. Figure 6-15 shows that SET 2 is preferred over SET 1 as $\mathrm{w}_{1}$ increases and SET 4 , containing $30 \%$ of unconventional power usage, is only selected as the optimal system when the cost attribute is not considered $\left(\mathrm{w}_{1}=0\right)$. This is simply because currently developed unconventional power generation methods and energy storage devices are usually costly even though they significantly decrease emission rates. Figure 6-16 shows that an increase in $\mathrm{w}_{2}$ has little effect on the overall utility score of SET 6. 


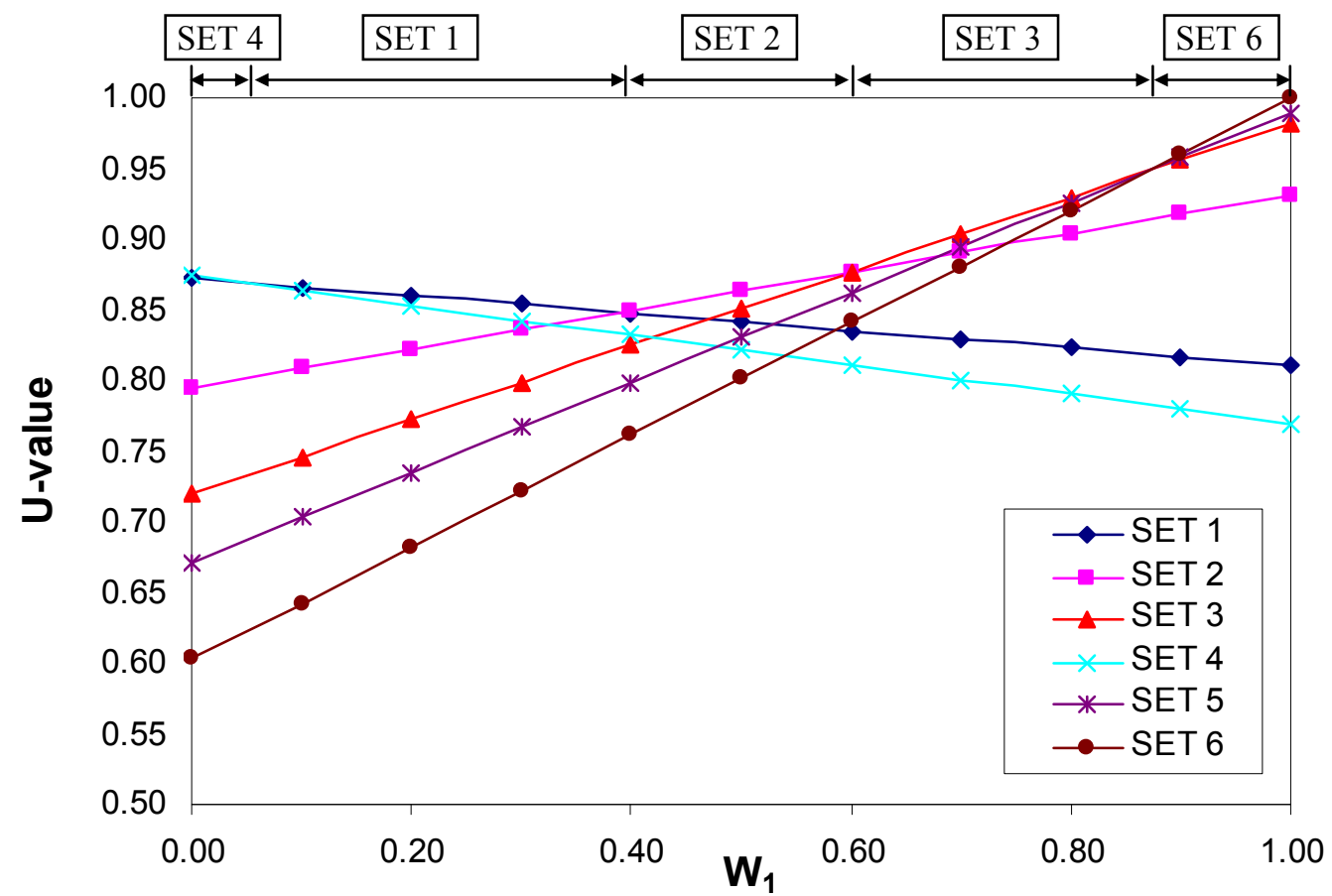

Figure 6-15. Optimal utility scores of the suggested systems when $\mathrm{W}_{1}$ is varied

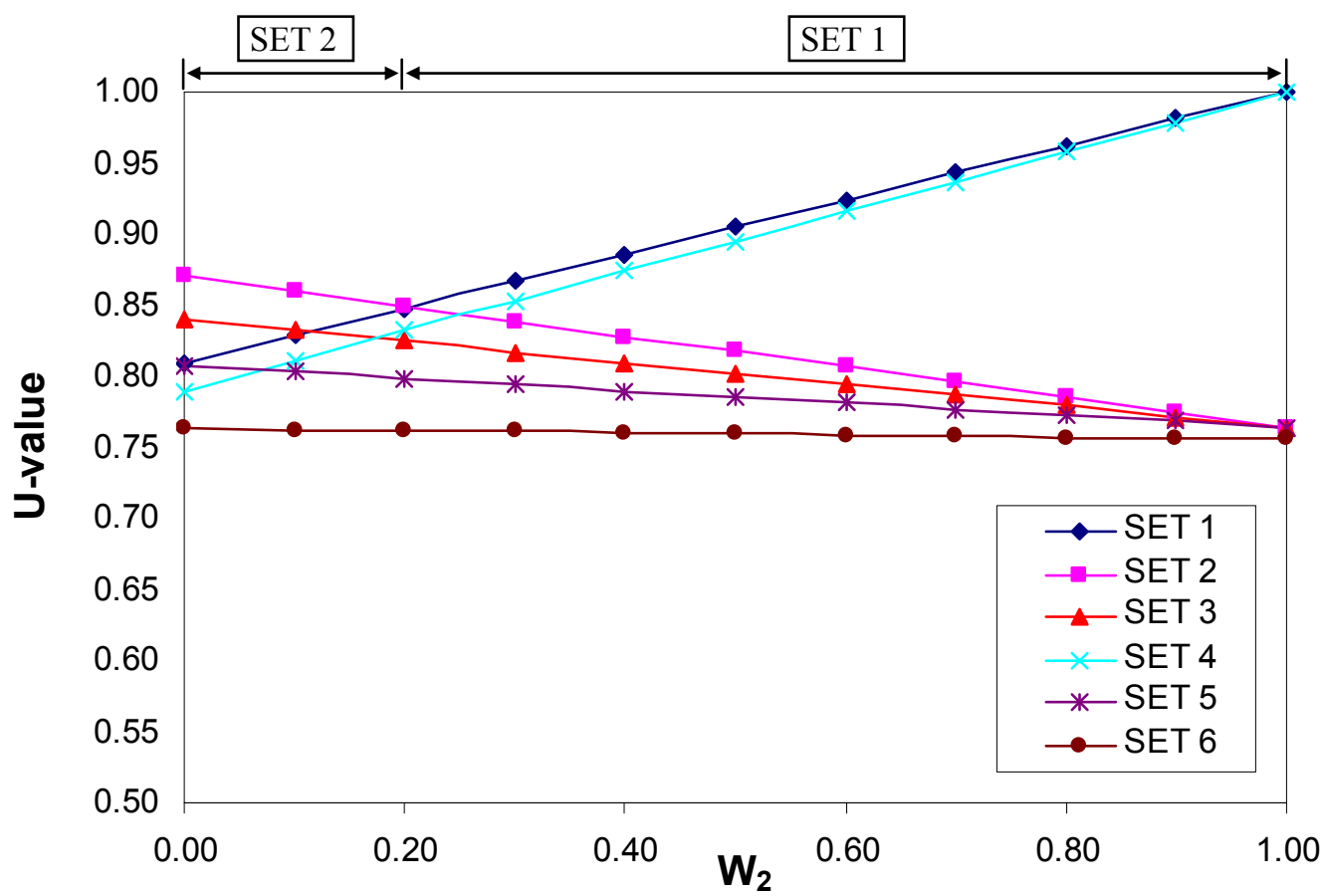

Figure 6-16. Optimal utility scores of the suggested systems when $\mathrm{W}_{2}$ is varied 


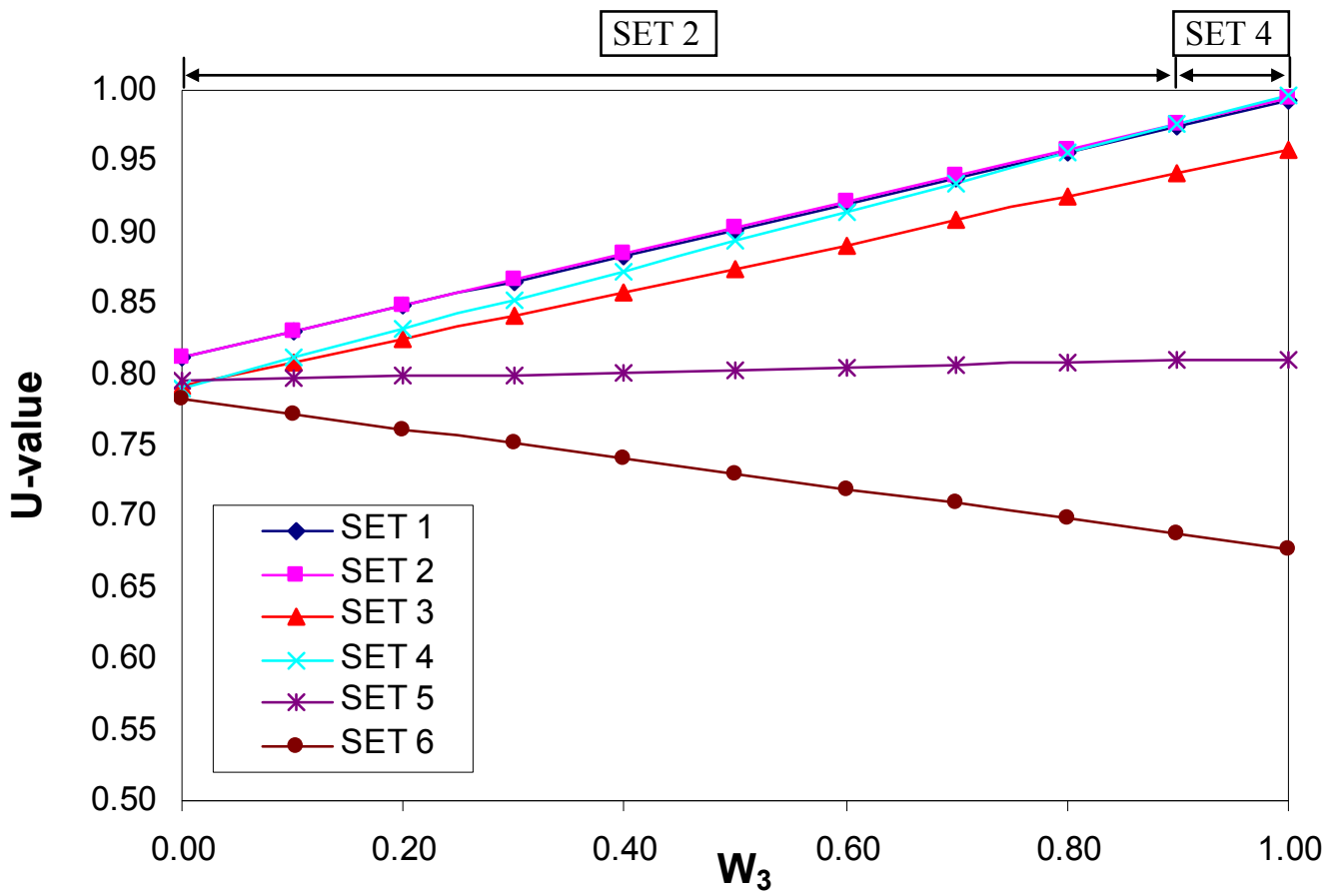

Figure 6-17. Optimal utility scores of the suggested systems when $\mathrm{W}_{3}$ is varied

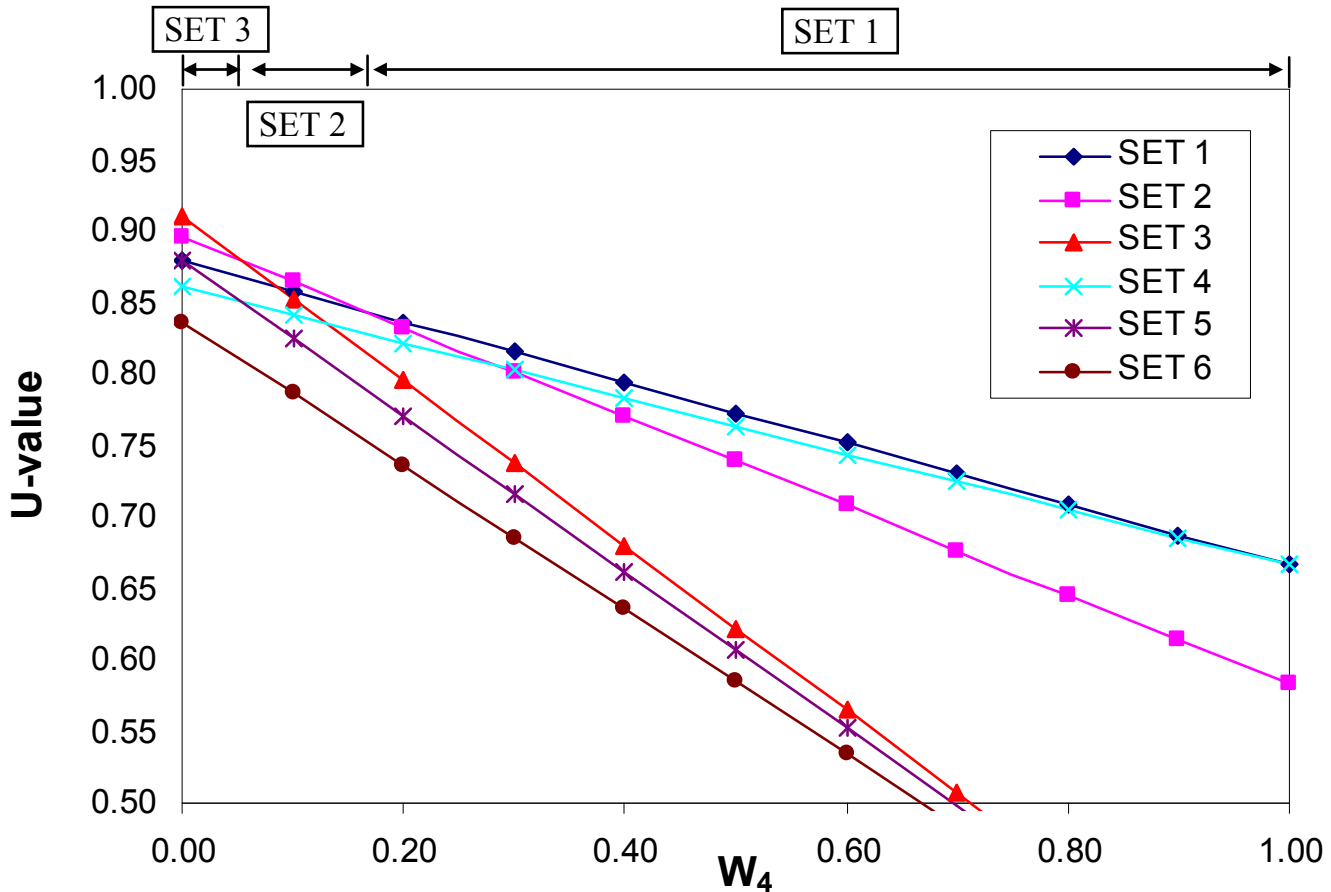

Figure 6-18. Optimal utility scores of the suggested systems when $\mathrm{W}_{4}$ is varied 


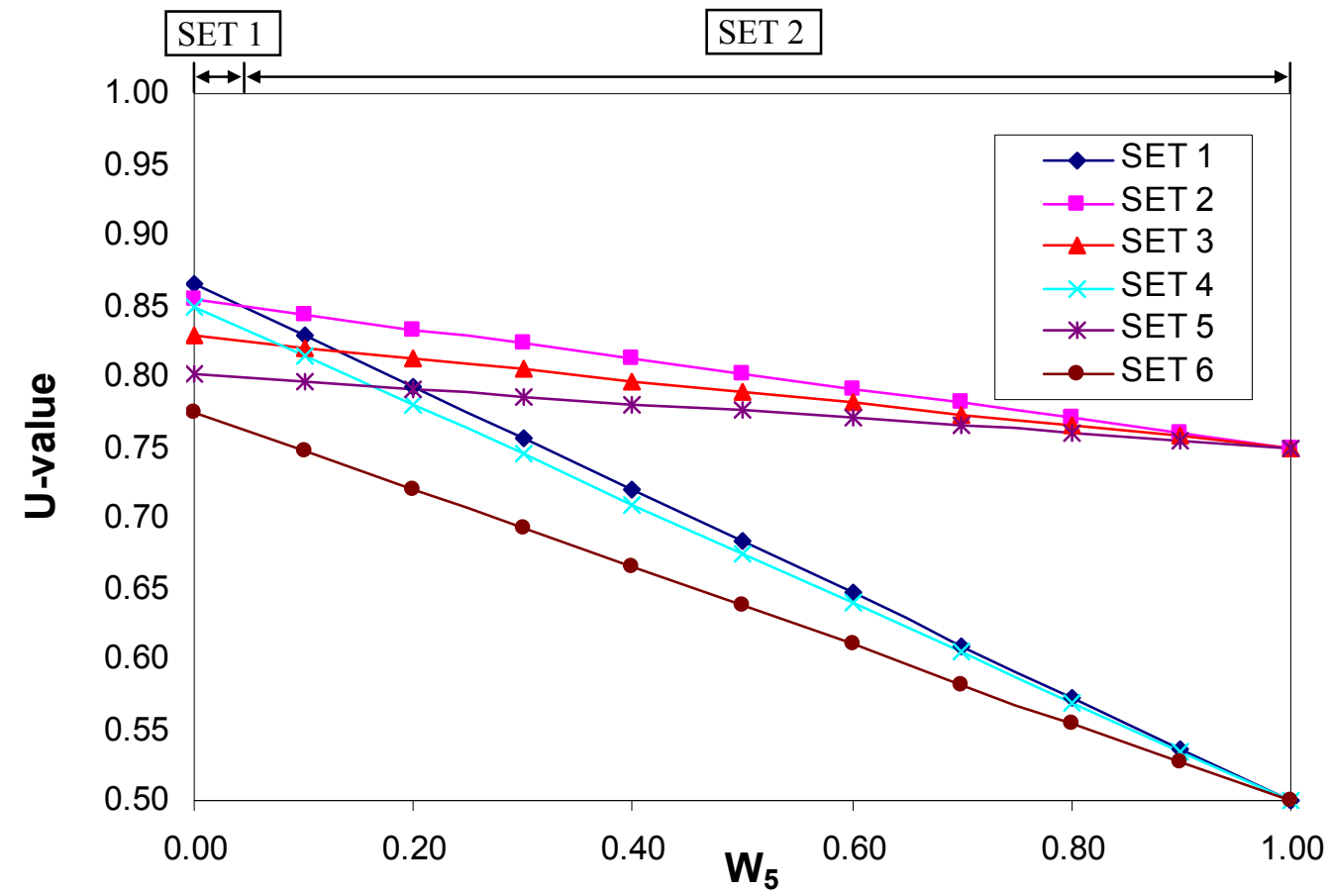

Figure 6-19. Optimal utility scores of the suggested systems when $\mathrm{W}_{5}$ is varied

Another way of displaying the results of the sensitivity analysis is shown in Figure 6-20 through Figure 6-29. These figures focus not on the relative overall utility score of the different systems but on the system selections themselves. This displaying method is more useful and intuitive when people want to know which system should be selected with a given weight combination. However, the drawback of using this method is these are only three-dimensional plots so two remaining weights should be fixed at zero. 


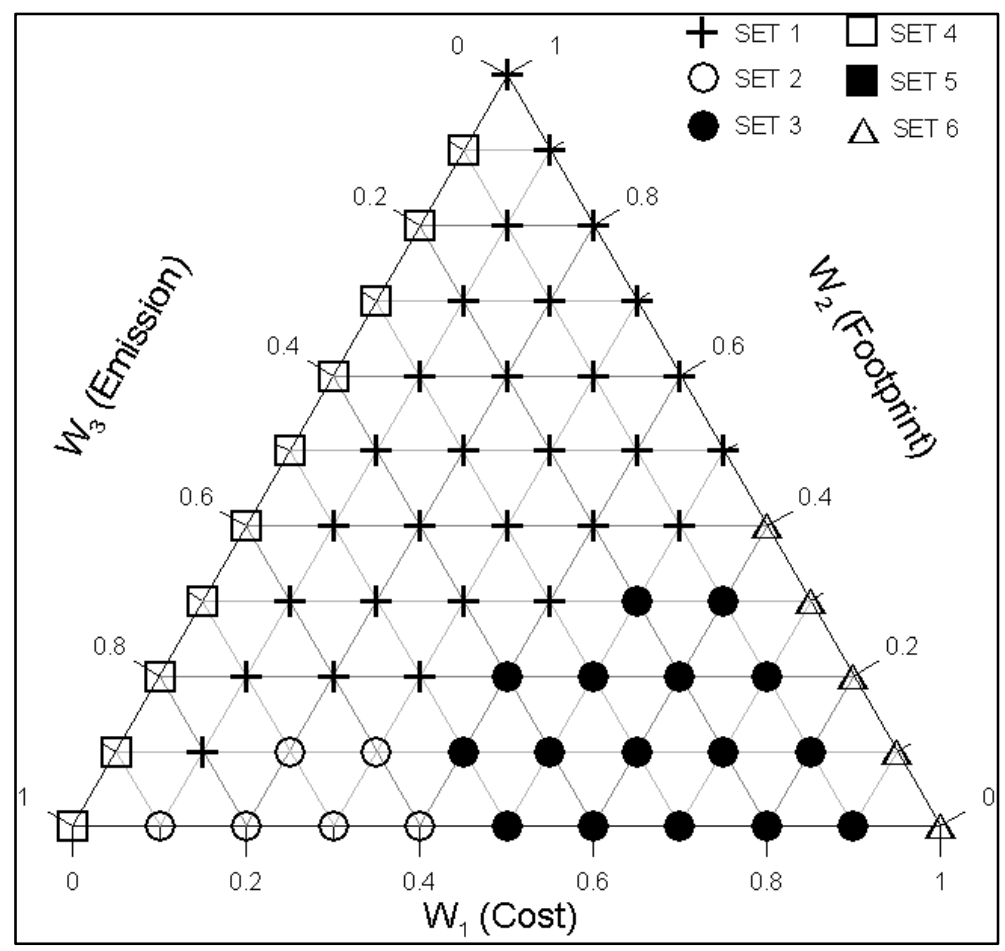

Figure 6-20. Optimal system selection as a function of $\mathrm{W}_{1}, \mathrm{~W}_{2}$, and $\mathrm{W}_{3}$

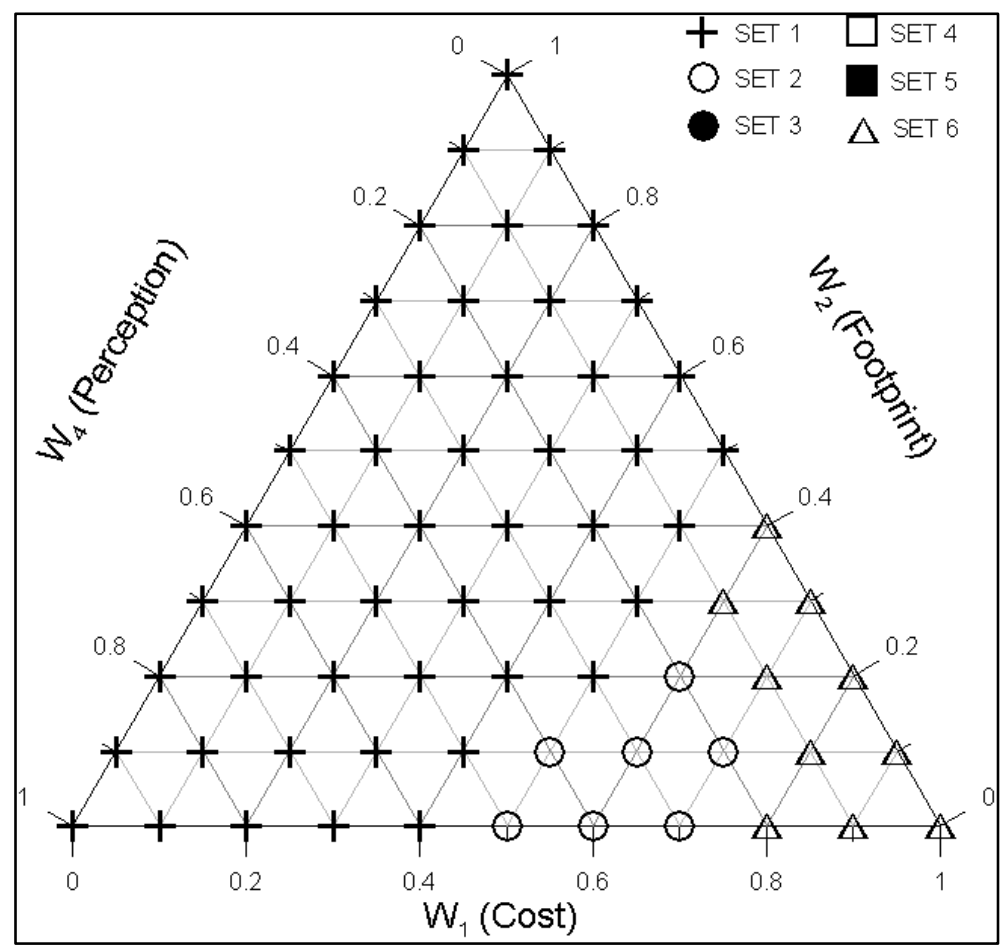

Figure 6-21. Optimal system selection as a function of $W_{1}, W_{2}$, and $W_{4}$ 


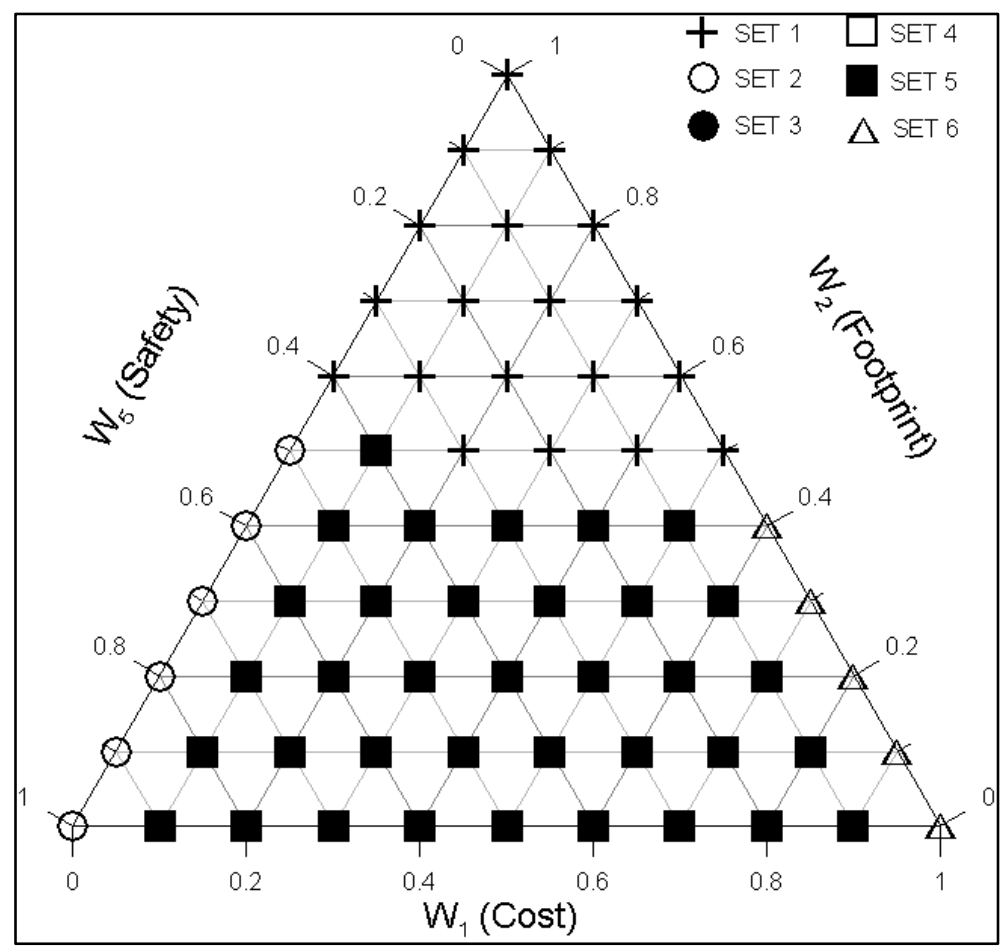

Figure 6-22. Optimal system selection as a function of $\mathrm{W}_{1}, \mathrm{~W}_{2}$, and $\mathrm{W}_{5}$

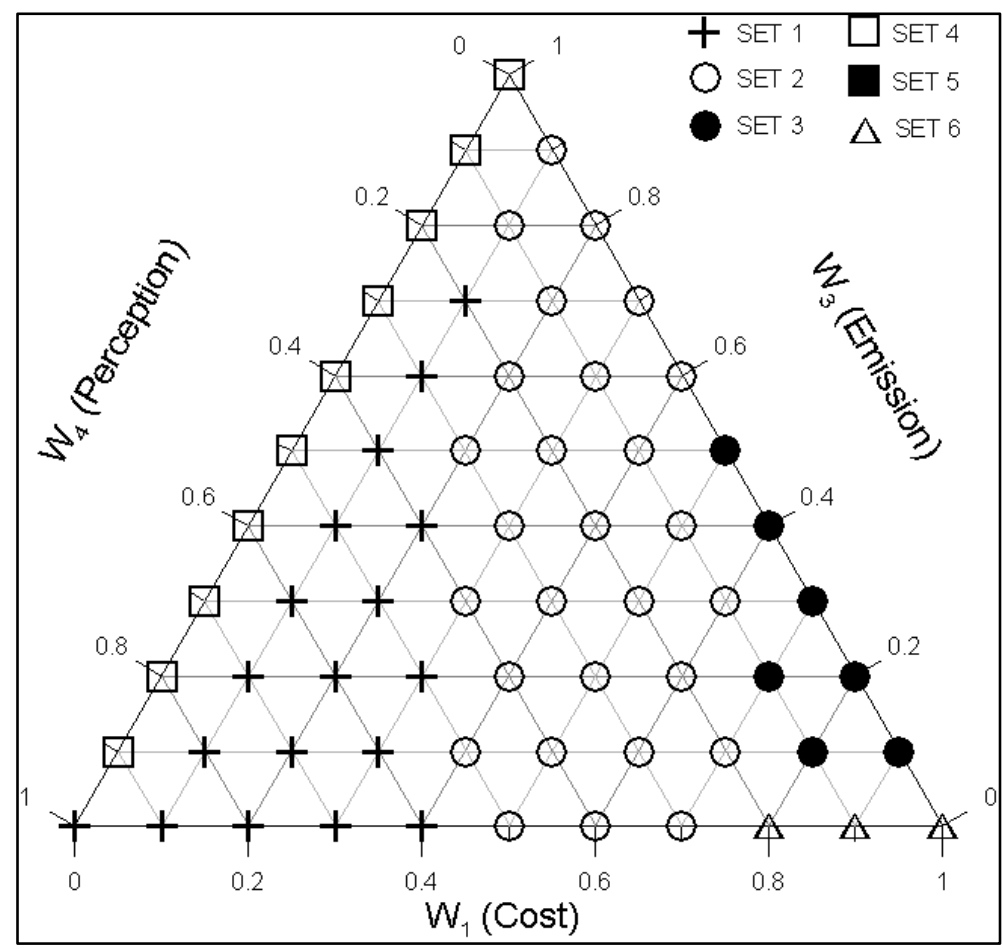

Figure 6-23. Optimal system selection as a function of $\mathrm{W}_{1}, \mathrm{~W}_{3}$, and $\mathrm{W}_{4}$ 


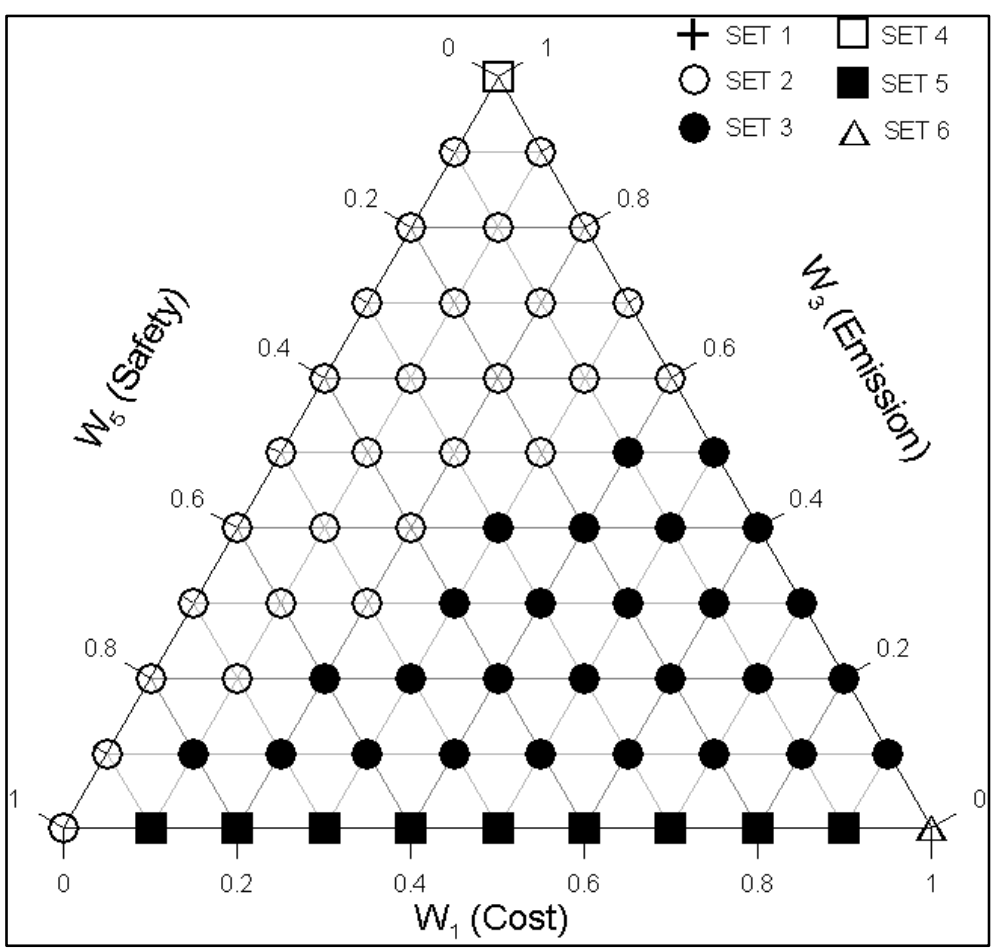

Figure 6-24. Optimal system selection as a function of $\mathrm{W}_{1}, \mathrm{~W}_{3}$, and $\mathrm{W}_{5}$

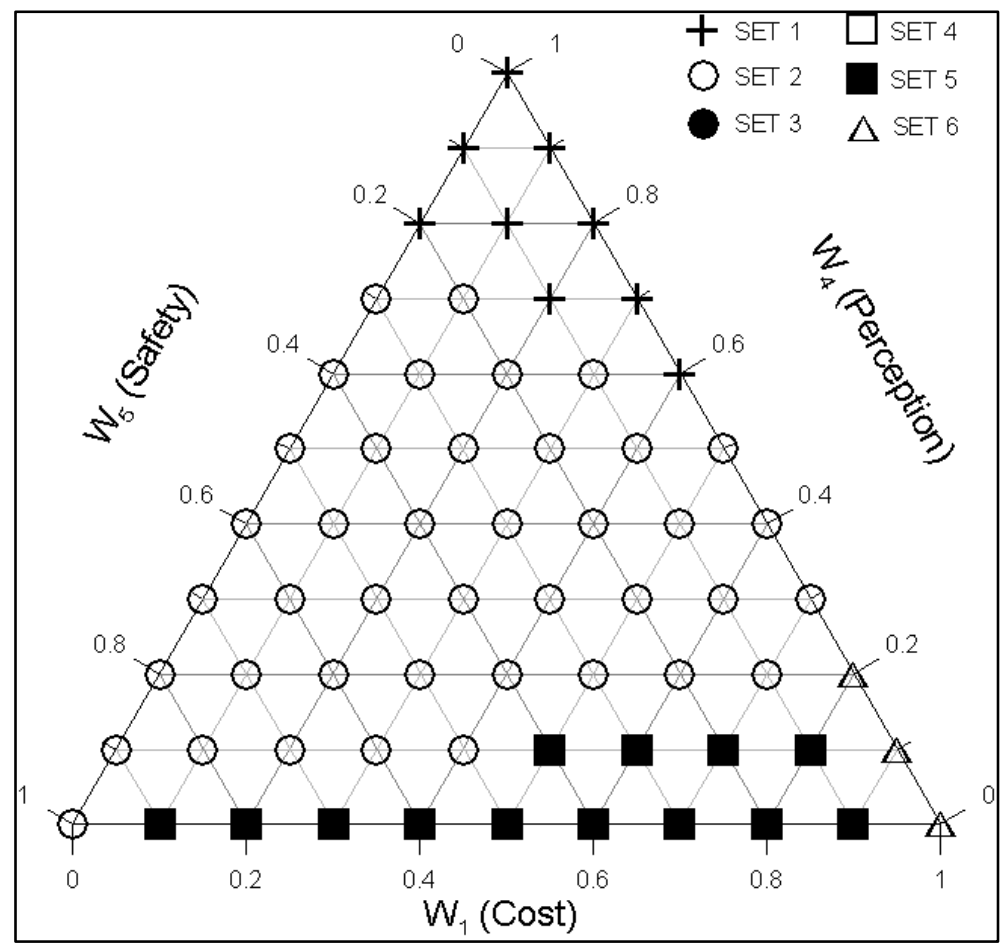

Figure 6-25. Optimal system selection as a function of $\mathrm{W}_{1}, \mathrm{~W}_{4}$, and $\mathrm{W}_{5}$ 


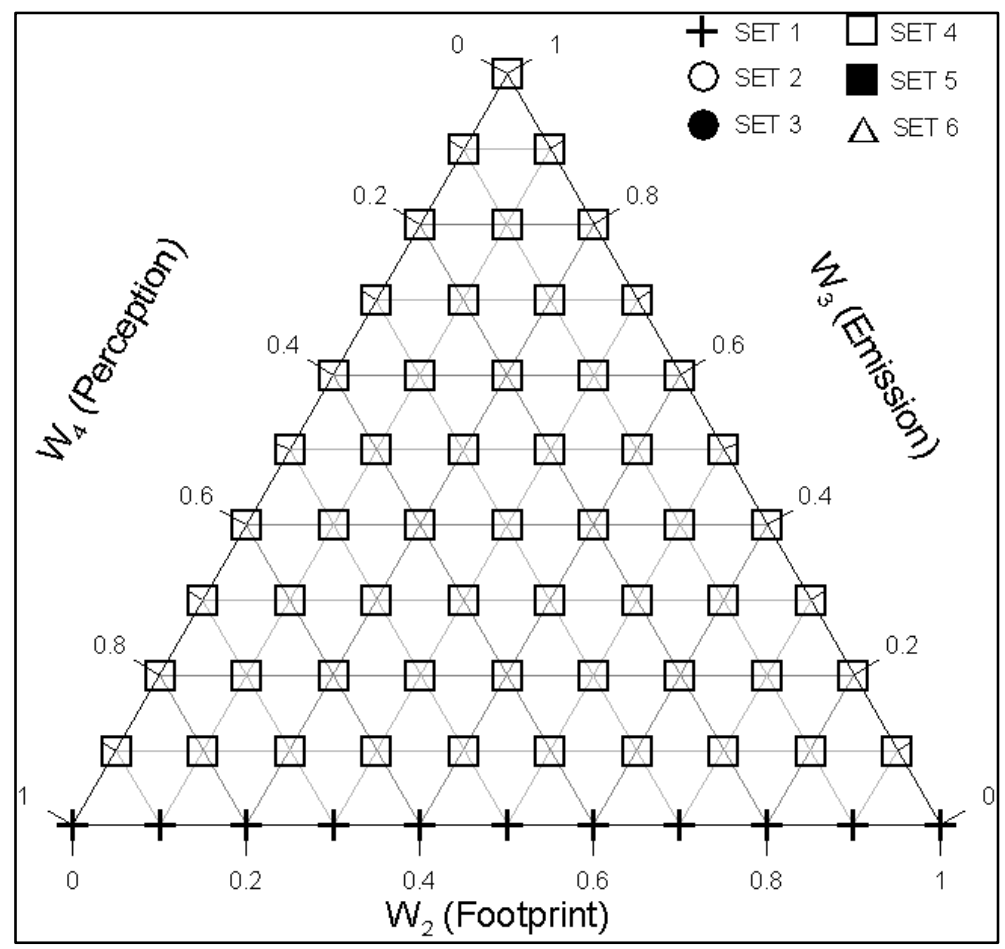

Figure 6-26. Optimal system selection as a function of $\mathrm{W}_{2}, \mathrm{~W}_{3}$, and $\mathrm{W}_{4}$

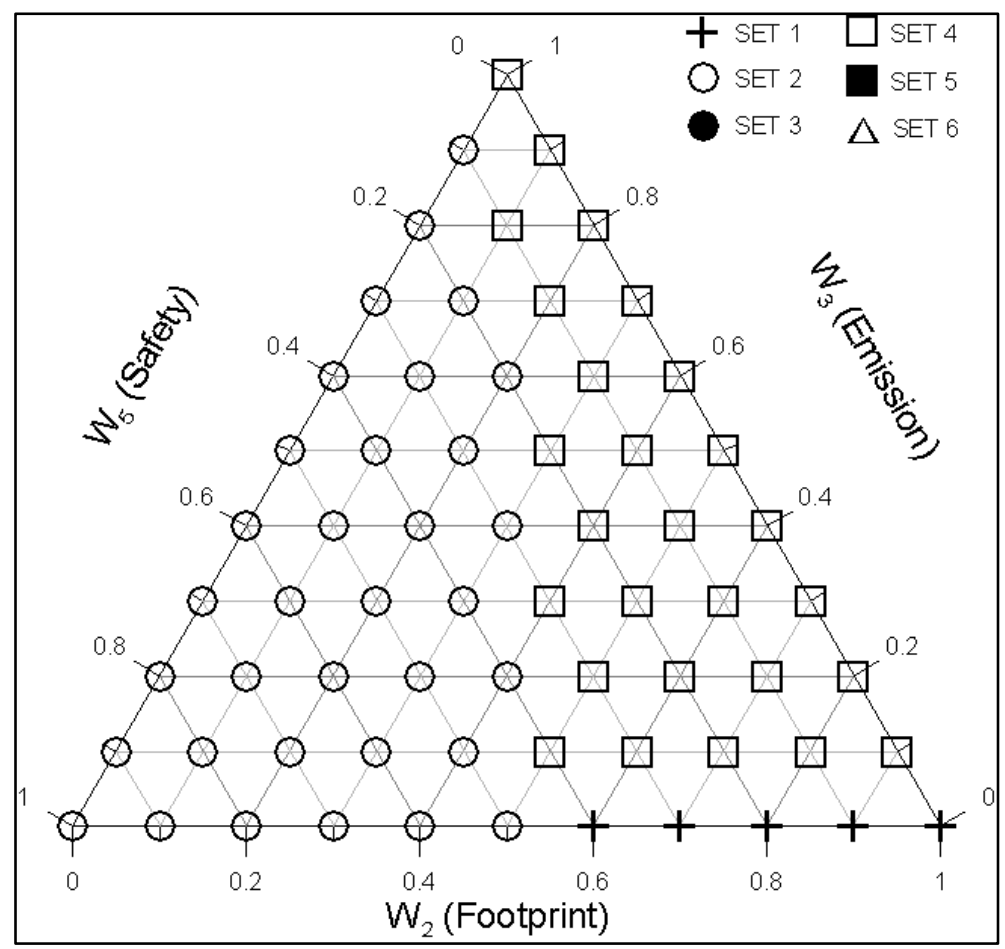

Figure 6-27. Optimal system selection as a function of $\mathrm{W}_{2}, \mathrm{~W}_{3}$, and $\mathrm{W}_{5}$ 


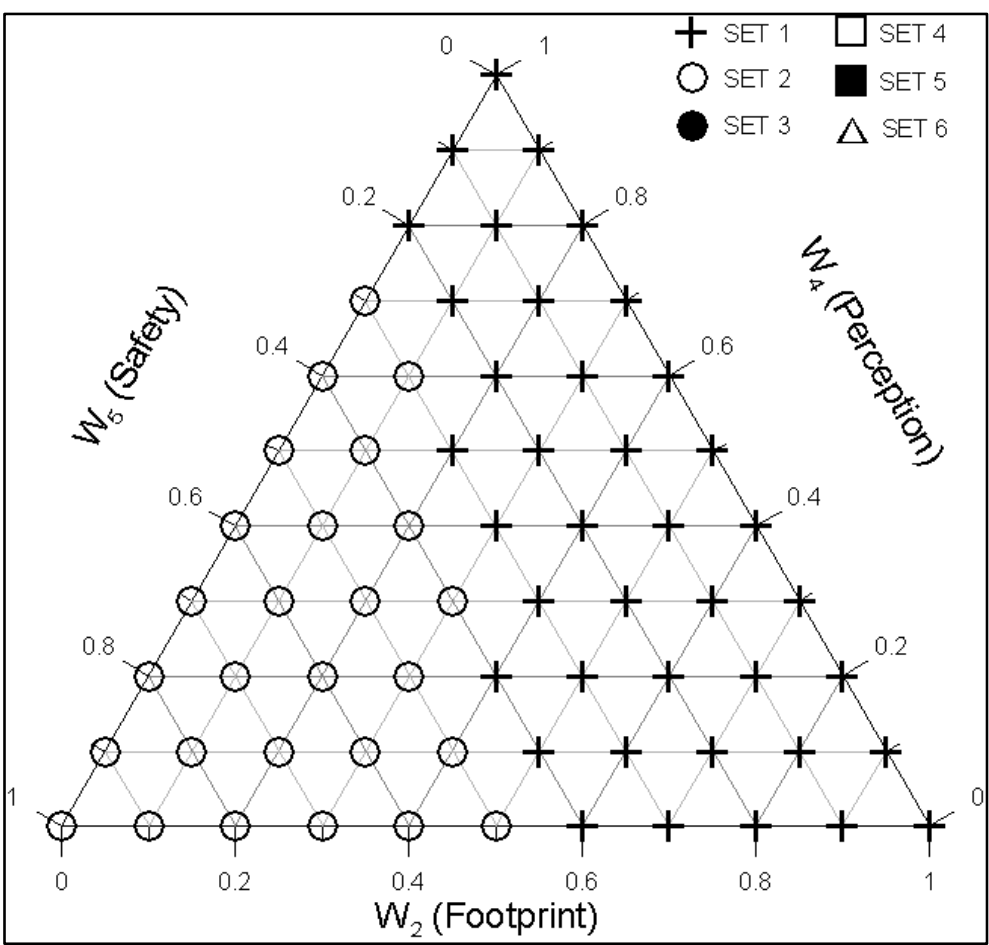

Figure 6-28. Optimal system selection as a function of $\mathrm{W}_{2}, \mathrm{~W}_{4}$, and $\mathrm{W}_{5}$

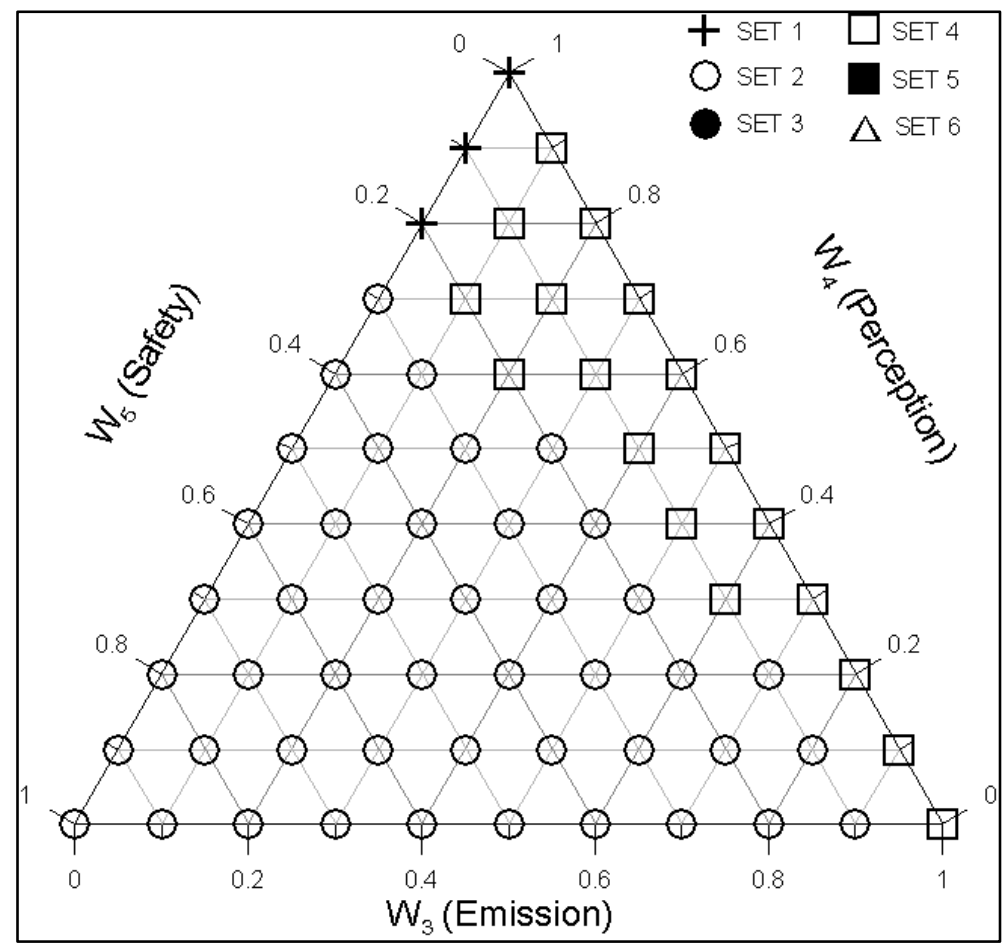

Figure 6-29. Optimal system selection as a function of $\mathrm{W}_{3}, \mathrm{~W}_{4}$, and $\mathrm{W}_{5}$ 


\subsubsection{Sensitivity Analysis for Uncertainty of Overall Attribute Scores}

In order to identify how much sensitive the overall utility score is to changes in the input attribute scores, overall attribute scores of two different systems (i.e., SET 1 and SET 6, which are the most and the least suggested optimal system) are varied from the original values with two different discrete weight combinations shown in Table 6-7. The variation of the cost, footprint, and emission attribute scores are $\pm 10 \%$ from the original values and the variation of other attribute scores (i.e., perception and safety) are upper and lower grade score from the original values. The input scores and the variation of the overall attribute scores being used in this sensitivity analysis are shown in Figure 6-30 and Figure 6-31. It is noted that the overall public perception score of SET 1 can't be varied to the upper grade score because the original score of this attribute is one, which is the possible maximum score can be assigned to this attribute. The possible maximum and minimum score should be considered for attributes using the ordinal scales such as solid/liquid emission, three perceptions, and safety.

Table 6-7. Weight combinations used in the sensitivity analysis

\begin{tabular}{|c|c|c|c|c|c|c|c|c|c|c|}
\hline Weight & Cost & Footprint & \multicolumn{4}{|c|}{ Emissions $\left(\mathrm{W}_{3}\right)$} & \multicolumn{3}{|c|}{ Perception $\left(\mathrm{W}_{4}\right)$} & Safety \\
no. & $\left(\mathrm{W}_{1}\right)$ & $\left(\mathrm{W}_{2}\right)$ & Air & $\mathrm{S} / \mathrm{L}$ & Noise & Gov. & Ind. & Public & \multirow{2}{*}{$\left(\mathrm{W}_{5}\right)$} & \\
\hline 1 & $1 / 9$ & $1 / 9$ & $1 / 9$ & $1 / 9$ & $1 / 9$ & $1 / 9$ & $1 / 9$ & $1 / 9$ & $1 / 9$ & Even \\
\hline 2 & 0.40 & 0.20 & $0.20 / 3$ & $0.20 / 3$ & $0.20 / 3$ & 0.05 & 0.05 & 0.05 & 0.05 & Base case \\
\hline
\end{tabular}

\begin{tabular}{|c|c|c|c|c|c|c|c|c|c|}
\hline \multirow{2}{*}{$\begin{array}{l}\text { Selected Technologies } \\
\text { in Each Subset }\end{array}$} & \multirow{2}{*}{$\begin{array}{l}\text { Total Cost } \\
\text { (\$) }\end{array}$} & \multirow{2}{*}{$\begin{array}{l}\text { Ecological } \\
\text { Footprint } \\
\text { (Acres) }\end{array}$} & \multicolumn{3}{|c|}{ Emissions } & \multicolumn{3}{|c|}{ Perceptions } & \multirow{2}{*}{$\begin{array}{l}\text { Safety } \\
\text { Value }\end{array}$} \\
\hline & & & Air & $\begin{array}{l}\text { Solid\& } \\
\text { Liquid }\end{array}$ & $\begin{array}{l}\begin{array}{l}\text { Noise } \\
\text { (TWA })\end{array} \\
\end{array}$ & Gov. & Ind. & Public & \\
\hline (1) Transportation: Low sulphur diesel truck w/tier III engine, w/noise suppressor & & & & & & 1.000 & 0.500 & 1.000 & 0.750 \\
\hline (2) Road construction: DURA-BASE from Composite Mat (rent) & $\$ 147,840$ & 1.515 & 0.976 & & 64.696 & 1.000 & 0.500 & 1.000 & 1.000 \\
\hline (3) Site preparation: Aluminum modules + driven piles & $\$ 226,261$ & 0.005 & 0.989 & & 76.265 & 1.000 & 0.500 & 1.000 & 0.500 \\
\hline (4) Rig type: LOC250 (CWD) & $\$ 173,800$ & & 0.985 & & 60.366 & 1.000 & 0.500 & 1.000 & 1.000 \\
\hline (5) Rig power (Conventional): Lean-burn natural gas engines w/noise suppressor & $\$ 70,354$ & & 0.918 & & 85.603 & 1.000 & 0.500 & 1.000 & 0.750 \\
\hline (6) Fuel type: Natural gas & $\$ 25,650$ & & - & & . & 1.000 & 0.500 & 1.000 & 0.750 \\
\hline (7) Rig power (Unconventional): Electric power from grid (10\%) & $\$ 3,840$ & 0.000 & 1.000 & & 0.000 & 0.500 & 1.000 & 1.000 & 1.000 \\
\hline (8) Energy storage: Flywheels & $\$ 30,000$ & 0.000 & & & & 0.500 & 1.000 & 1.000 & 0.750 \\
\hline (9) Drilling tech.: Managed pressure drilling w/noise suppressor & $\$ 193,500$ & & & & 94.100 & 0.750 & 0.750 & 1.000 & 1.000 \\
\hline (10) Fluid type: Water-based muds & $\$ 47,940$ & & & & & 1.000 & 1.000 & 1.000 & 1.000 \\
\hline (11) Waste mgmt.: Closed loop + containers + solid control equip.* & $\$ 27,000$ & 0.000 & & 1.000 & & 1.000 & 0.500 & 1.000 & 0.750 \\
\hline (12) Cuttings mgmt.: Cuttings injection & $\$ 45,000$ & & & 1.000 & & 1.000 & 0.500 & 1.000 & 0.750 \\
\hline \multicolumn{10}{|l|}{ (13) Noise reduction: N/A } \\
\hline Overall Attribute Scores ( $\Sigma$ or minimum value) & $\$ 991,184$ & 1.520 & 4.868 & 2.000 & 381.030 & 0.500 & 0.500 & 1.000 & 0.500 \\
\hline Single Attribute Utility Values (Original) & 0.811 & 0.966 & 0.768 & 1.000 & 0.988 & 0.500 & 0.500 & 1.000 & 0.500 \\
\hline Used Upper Bound Scores & $\$ 1,090,302.68$ & 1.67 & 5.35 & 2.00 & 419.13 & 0.75 & 0.75 & 1.00 & 0.75 \\
\hline Used Lower Bound Scores & $\$ 892,065.83$ & 1.37 & 4.38 & 1.80 & 342.93 & 0.25 & 0.25 & 0.75 & 0.25 \\
\hline
\end{tabular}

Figure 6-30. Input values and variation of the overall attribute scores of SET 1 


\begin{tabular}{|c|c|c|c|c|c|c|c|c|c|}
\hline \multirow{2}{*}{$\begin{array}{l}\text { Selected Technologies } \\
\text { in Each Subset }\end{array}$} & \multirow{2}{*}{$\begin{array}{l}\text { Total Cost } \\
\text { (\$) }\end{array}$} & \multirow{2}{*}{$\begin{array}{c}\text { Ecological } \\
\text { Footprint } \\
\text { (Acres) }\end{array}$} & \multicolumn{3}{|c|}{ Emissions } & \multicolumn{3}{|c|}{ Perceptions } & \multirow{2}{*}{$\begin{array}{l}\text { Safety } \\
\text { Value }\end{array}$} \\
\hline & & & Air & $\begin{array}{l}\text { Solid\& } \\
\text { Liquid }\end{array}$ & $\begin{array}{l}\text { Noise } \\
\text { (TWA }\end{array}$ & Gov. & Ind. & Public & \\
\hline \begin{tabular}{|l} 
(1) Transportation: Coventional diesel truck \\
\end{tabular} & & & & & & 0.250 & 1.000 & 0.250 & 0.750 \\
\hline (2) Road construction: DURA-BASE from Composite Mat (rent) & $\$ 132,000$ & 1.515 & 0.964 & & 82.870 & 1.000 & 0.500 & 1.000 & 1.000 \\
\hline (3) Site preparation: DURA-BASE from Composite Mat (rent) & $\$ 90,000$ & 1.033 & 0.976 & & 79.945 & 0.750 & 0.750 & 0.750 & 1.000 \\
\hline (4) Rig type: LOC250 (CWD) & $\$ 167,000$ & & 0.977 & & 77.458 & 1.000 & 0.500 & 1.000 & 1.000 \\
\hline (5) Rig power (Conventional): Internal combustion engine & $\$ 50,000$ & & 0.338 & & 107.998 & 0.500 & 1.000 & 0.500 & 0.750 \\
\hline (6) Fuel type: Conventional diesel & $\$ 45,600$ & & 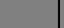 & & & 0.500 & 1.000 & 0.500 & 0.500 \\
\hline (7) Rig power (Unconventional): N/A ( $0 \%)$ & so & 0.000 & 1.000 & & 0.000 & 0.250 & 1.000 & 0.250 & 1.000 \\
\hline (8) Energy storage: N/A & so & 0.000 & & & & 0.250 & 1.000 & 0.250 & 1.000 \\
\hline (9) Drilling tech.: Underbalanced drilling w/noise suppressor & $\$ 184,500$ & . & & & 95.700 & 0.750 & 0.750 & 0.750 & 0.750 \\
\hline (10) Fluid type: Water-based muds & $\$ 47,940$ & & & & & 1.000 & 1.000 & 1.000 & 1.000 \\
\hline (11) Waste mgmt.: Lined reserve pit + solid control equip.* & $\$ 18,000$ & 0.037 & & 0.500 & & 0.750 & 0.750 & 0.750 & 0.500 \\
\hline (12) Cuttings mgmt:: Cuttings injection & $\$ 45,000$ & & & 1.000 & & 1.000 & 0.500 & 1.000 & 0.750 \\
\hline \multicolumn{10}{|l|}{ (13) Noise reduction: N/A } \\
\hline Overall Attribute Scores ( $\Sigma$ or minimum value) & $\$ 780,040$ & 2.585 & 4.254 & 1.500 & 443.971 & 0.250 & 0.500 & 0.250 & 0.500 \\
\hline Single Attribute Utility Values & 0.935 & 0.756 & 0.613 & 0.600 & 0.820 & 0.250 & 0.500 & 0.250 & 0.500 \\
\hline Used Upper Bound Scores & $\$ 858,044.00$ & 2.84 & $\begin{array}{ll}4.68 \\
383\end{array}$ & $\begin{array}{ll}1.65 \\
1.35\end{array}$ & \begin{tabular}{|l|l}
488.37 \\
399.57
\end{tabular} & \begin{tabular}{ll|l}
0.50 \\
0.00
\end{tabular} & \begin{tabular}{ll|l}
0.75 \\
0.25
\end{tabular} & 0.50 & 0.75 \\
\hline
\end{tabular}

Figure 6-31. Input values and variation of the overall attribute scores of SET 6

Figure 6-32 through Figure 6-39 show the sensitiveness of the input attribute scores of the two systems with two discrete weight combinations given in Table 6-7. In Figure 6-32, for example, since steeper slope indicates more sensitive attribute, the air emission attribute seems to be the most sensitive attribute among the nine attributes. In Figure 6-33, however, perception and safety attributes seem to change the overall utility score of SET 1 more than other attributes. This is because the cost, footprint, and emission attributes vary by only $\pm 10 \%$ from the original values while perception and safety attributes vary by about $20 \% \sim 100 \%$ from the original values due to the grade score scale (i.e., $0,0.25,0.50,0.75,1.00)$.

The weight factor assigned to each attribute is very important element when identifying the sensitiveness of input attribute scores. In Figure 6-34, for example, the cost attribute seems to be the most sensitive attribute for SET 1, which is not the same result shown in Figure 6-32. This is simply because the weight assigned to each attribute is different between these two figures. The weight assigned to the cost attribute is 1/9 in Figure 6-32 while the weight is 0.40 in Figure 6-34. The noise emission attribute seems to be the most sensitive attribute for SET 6 with 'Even' weight combination as shown in Figure 6-36. It is indicated that since the noise attribute utility curve is not a linear, the result of the variation $( \pm 10 \%)$ does not seem to be symmetrical from the original value. 


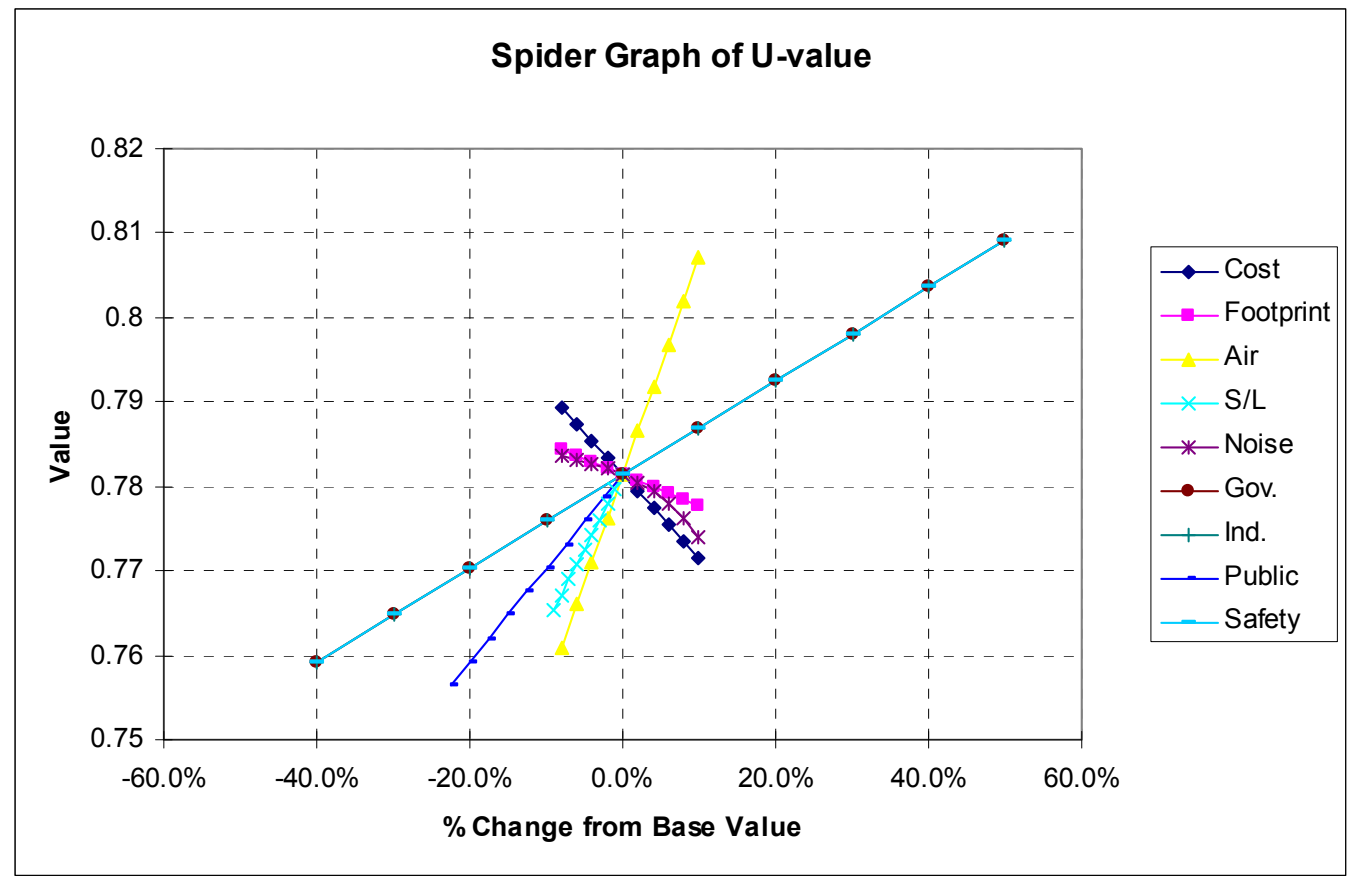

Figure 6-32. Spider graph for SET 1 with 'Even' weight combination in Table 6-7

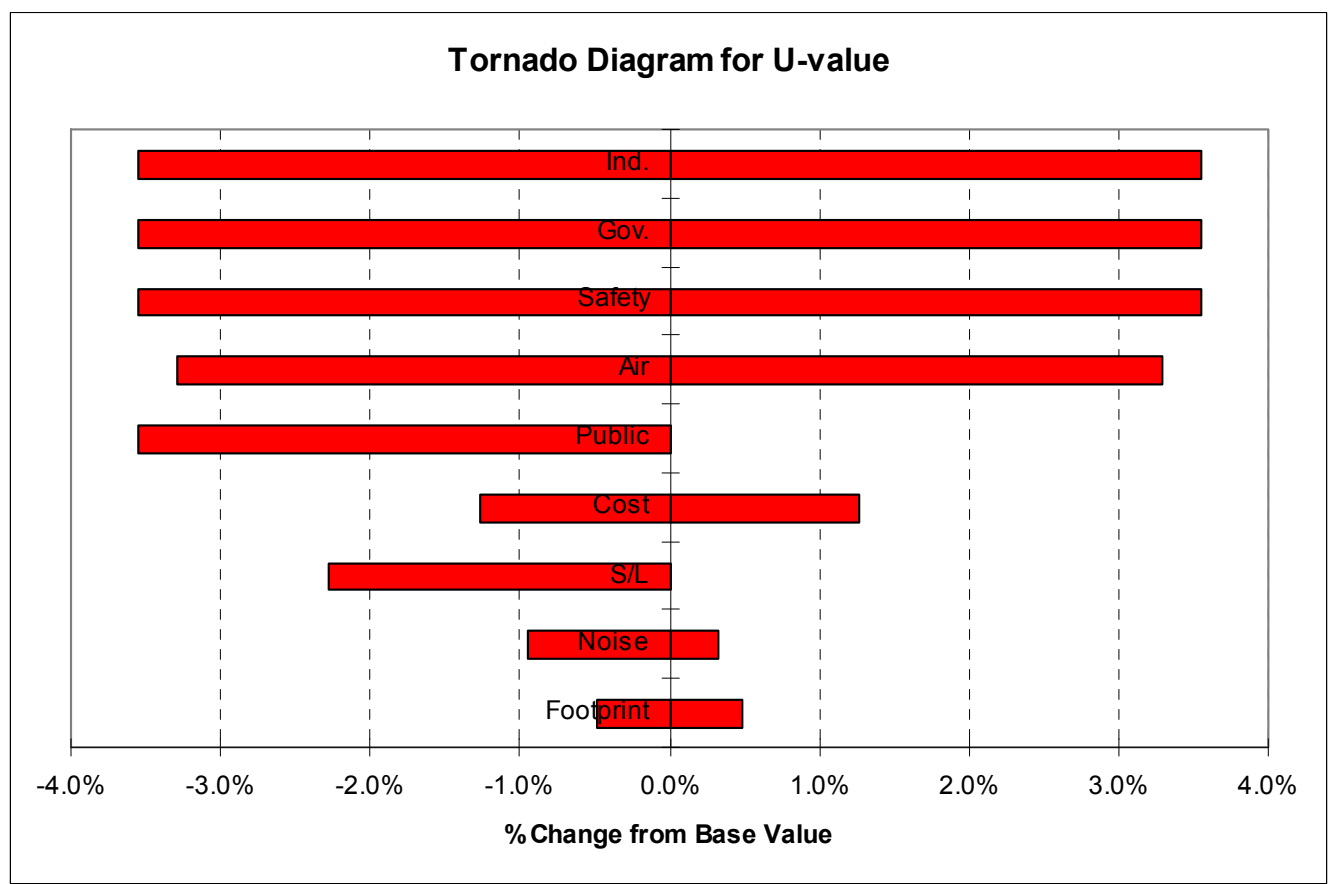

Figure 6-33. Tornado diagram for SET 1 with ‘Even’ weight combination 


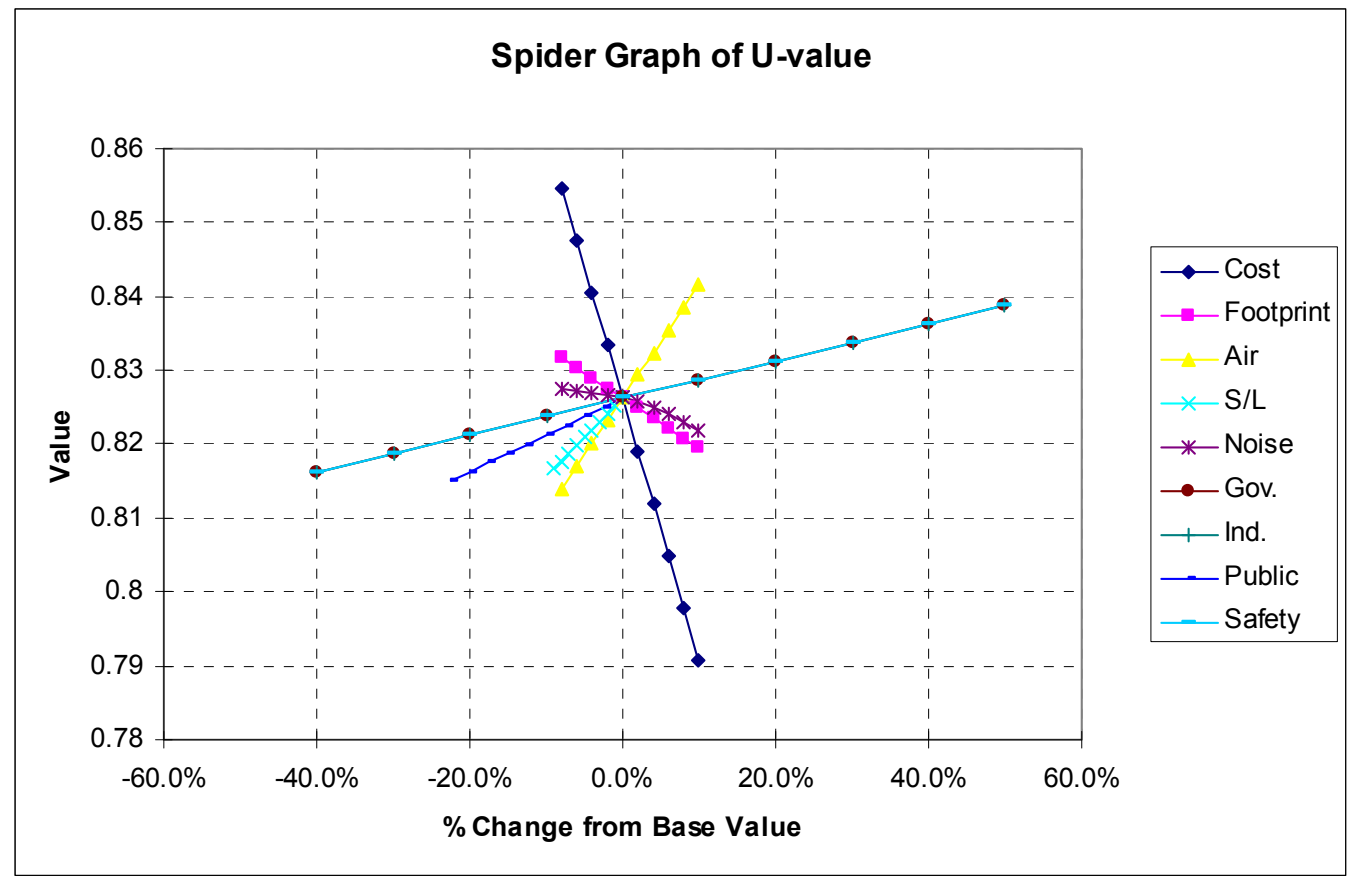

Figure 6-34. Spider graph for SET 1 with 'Base' weight combination in Table 6-7

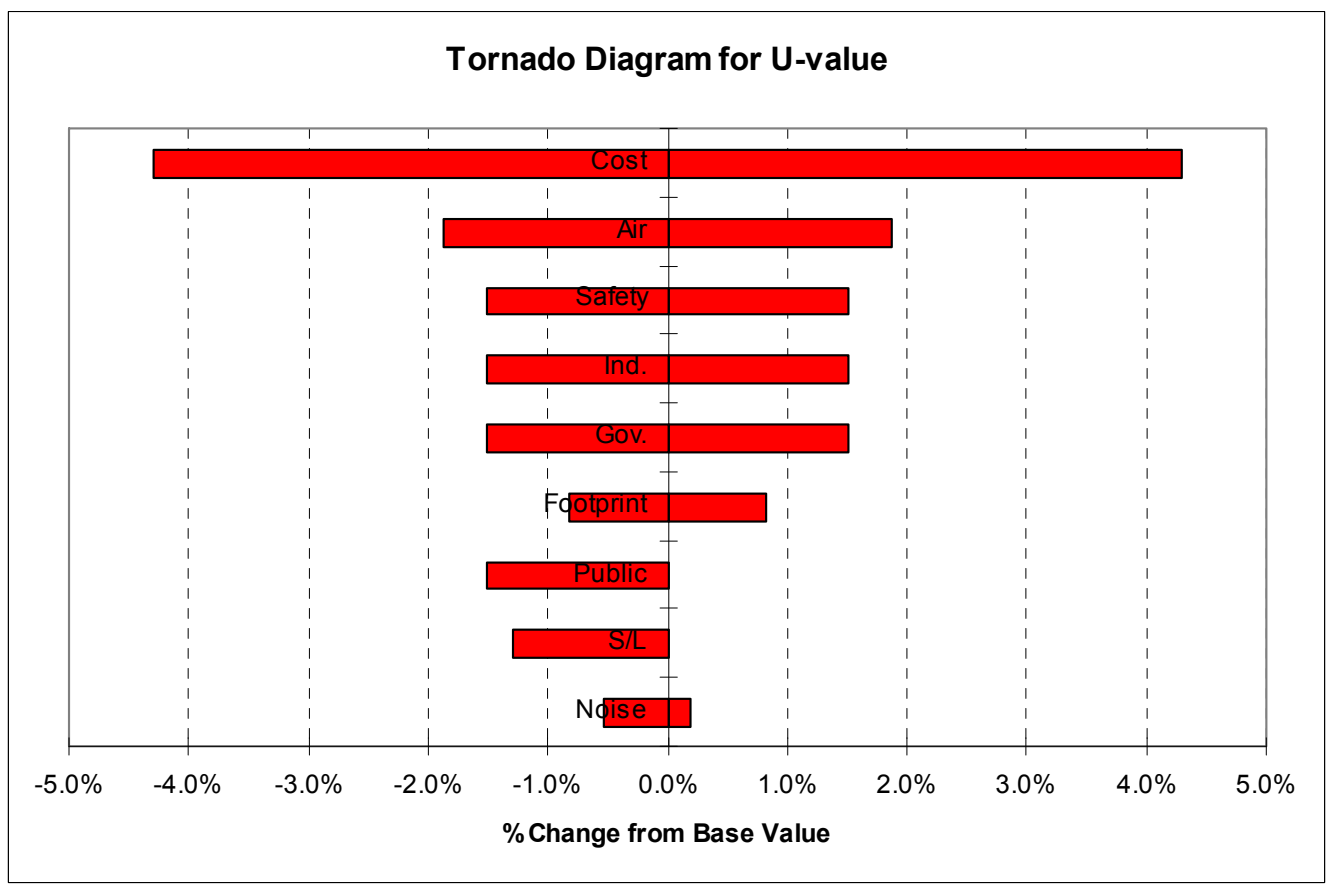

Figure 6-35. Tornado diagram for SET 1 with 'Base' weight combination 


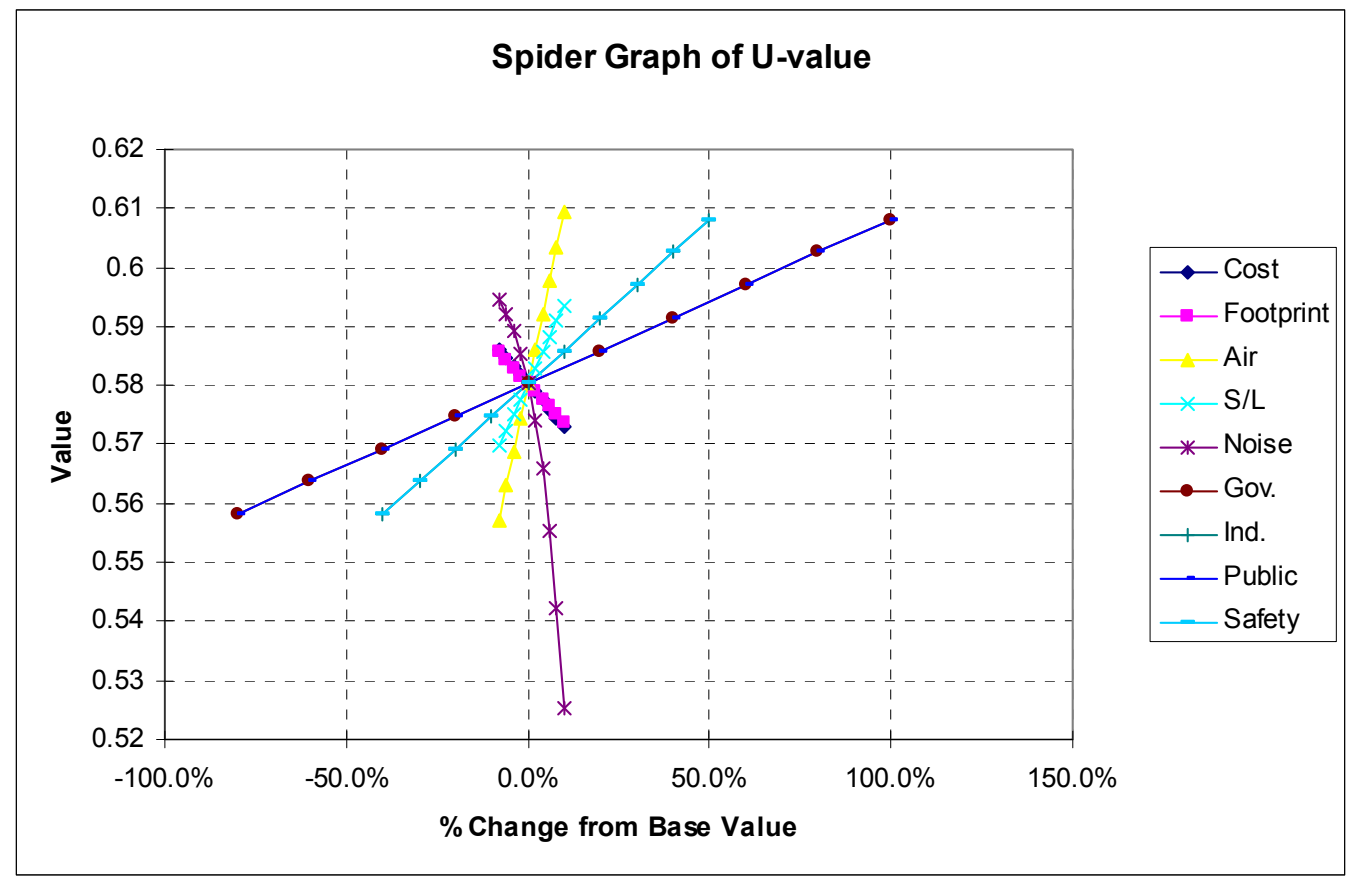

Figure 6-36. Spider graph for SET 6 with 'Even' weight combination in Table 6-7

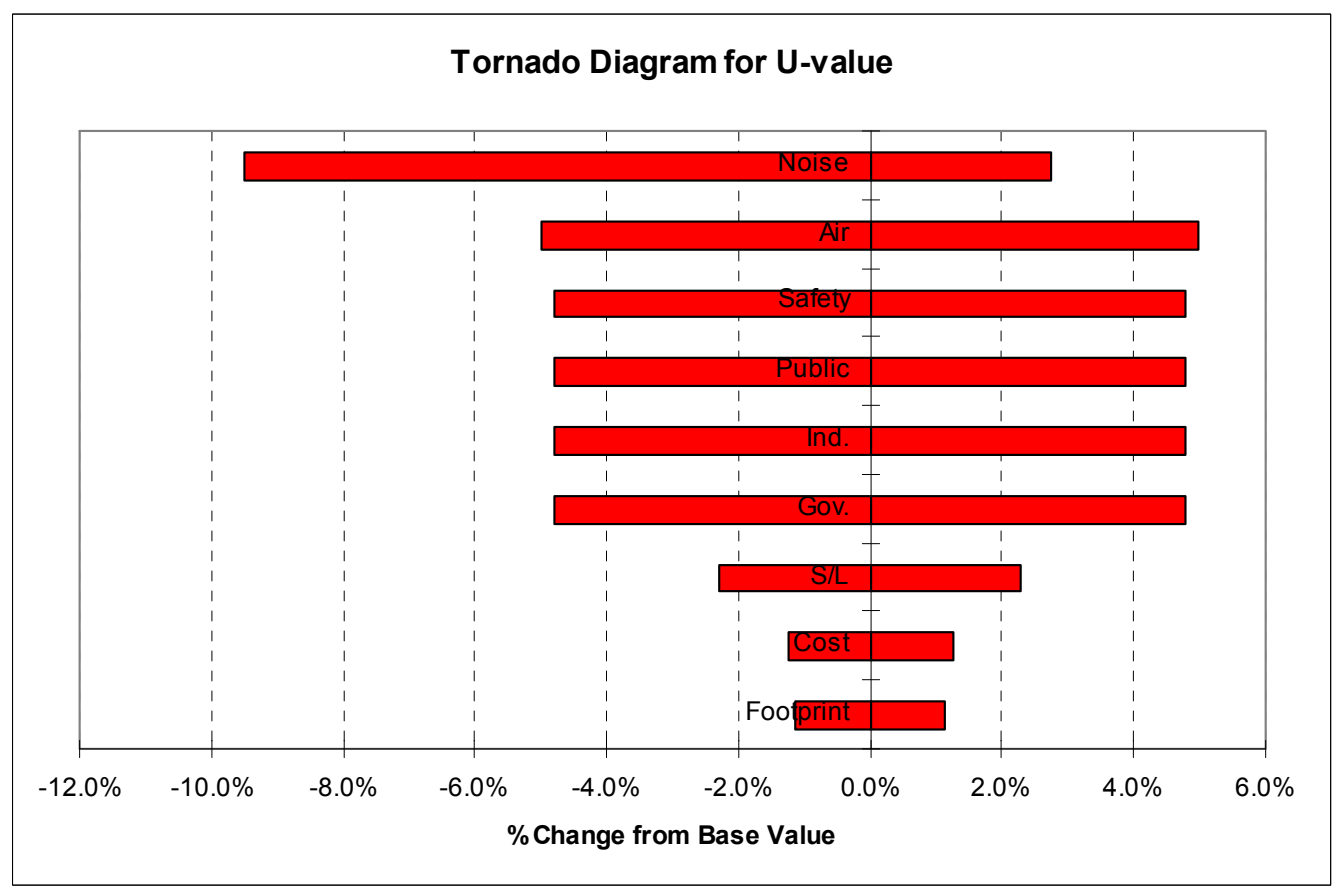

Figure 6-37. Tornado diagram for SET 6 with ‘Even' weight combination 


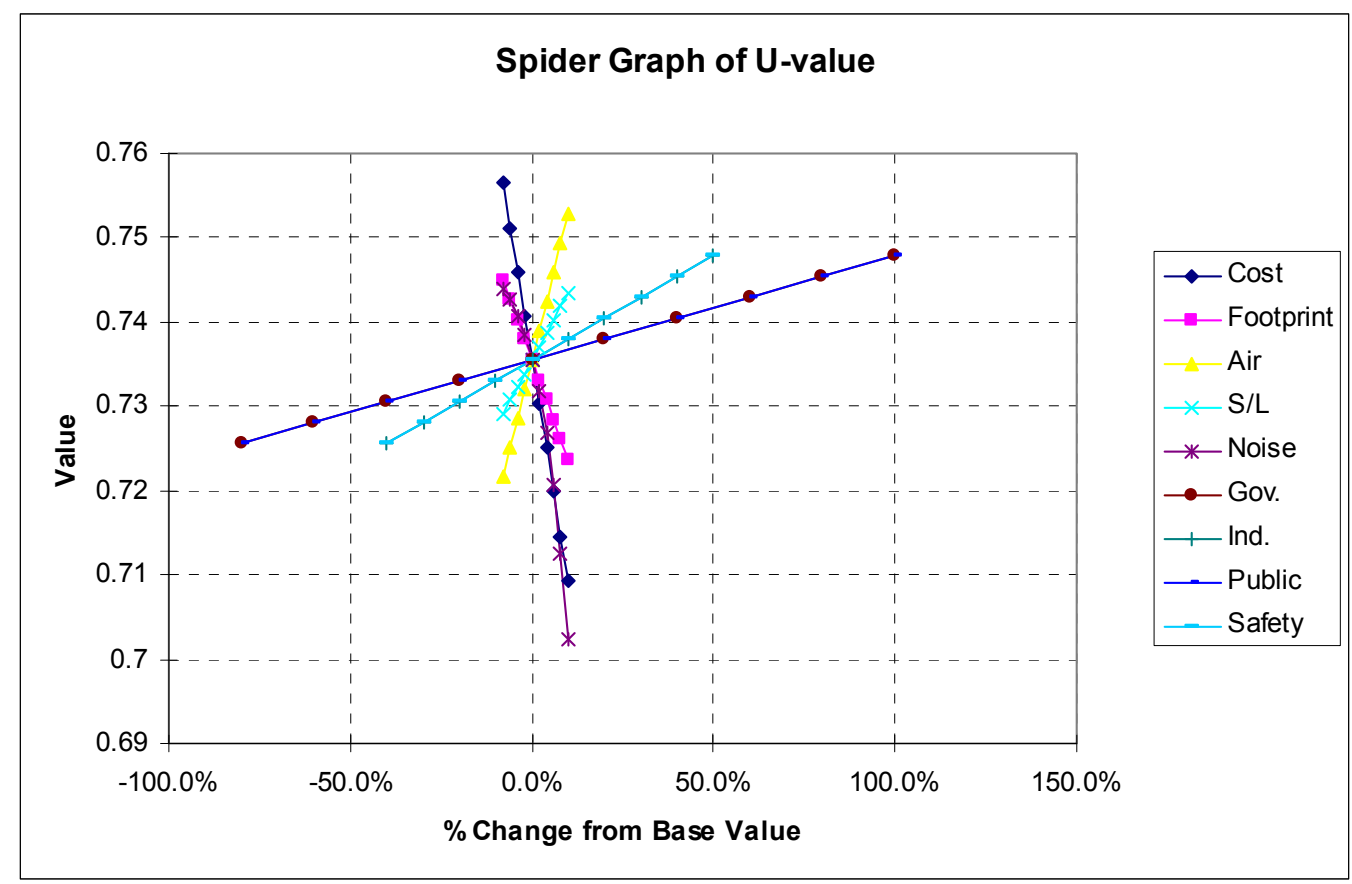

Figure 6-38. Spider graph for SET 6 with 'Base' weight combination in Table 6-7

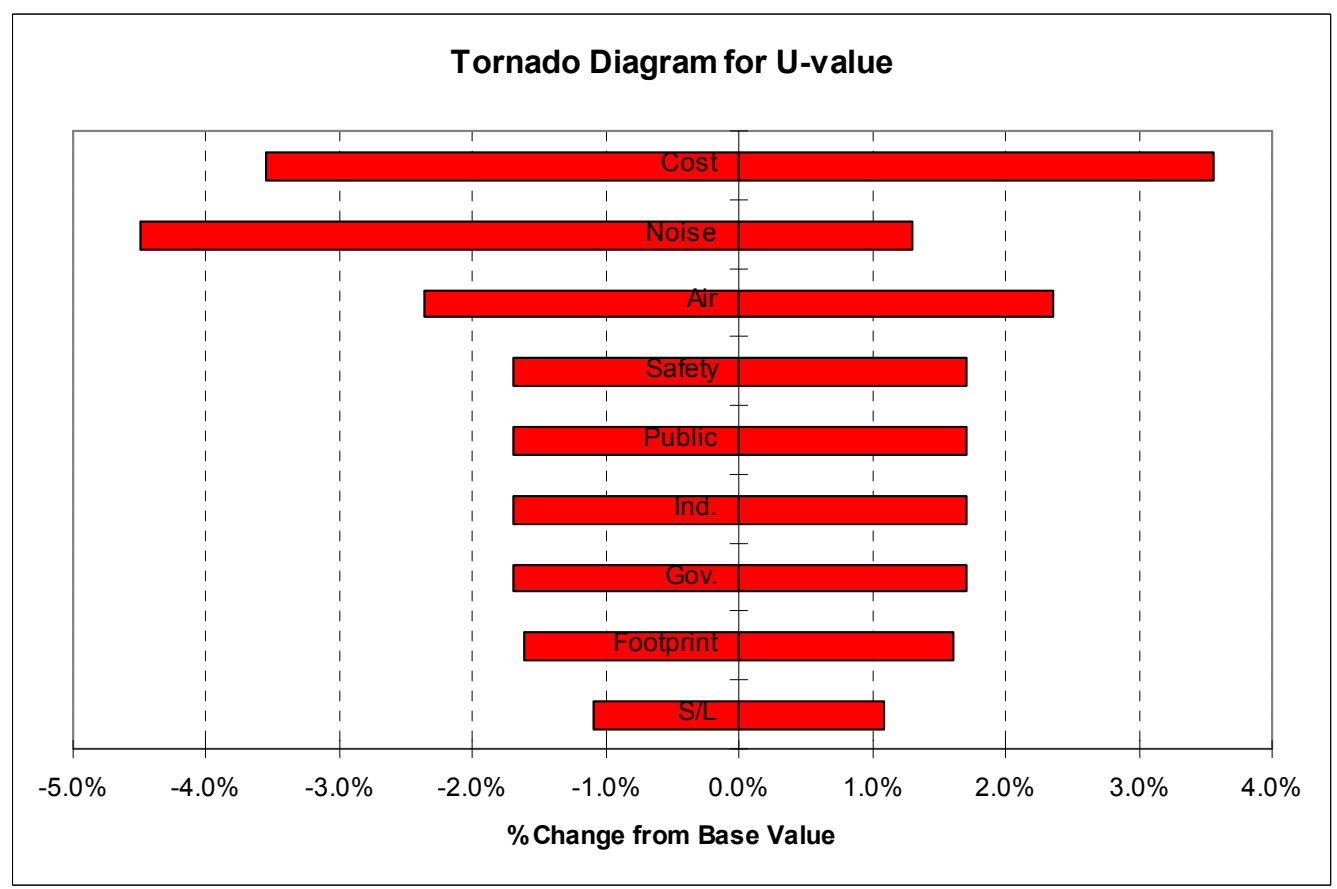

Figure 6-39. Tornado diagram for SET 6 with 'Base' weight combination 
In summary, if weight factors are evenly distributed to each attribute, air emission score and noise emission score are the most sensitive inputs for SET 1 and SET 6 as shown in Figure 6-32 and Figure 6-36, respectively. On the other hand, if weight factors are not evenly distributed to each attribute, the most sensitive input attribute can be identified after running the sensitivity analysis described in this section.

\subsection{Lessons Learned}

The knapsack optimization routine (APPENDIX B) was initially run for each weight combination in this case study. However, the critical issue arose while conducting the sensitivity analysis. Using the branch-and-bound optimization algorithm given in "Microsoft Excel Solver", it was not always able to find the global optimal solution. In some cases "Microsoft Excel Solver" was trapped at a local optimal solution. Therefore, in order to always get the global optimal solution (i.e., retain system with maximum overall utility score), an exhaustive search optimization was used in this study, but the complexity of the optimization problem grew rapidly with the number of decision variables. For example, once the number of potential systems was greater than a million, it was not reasonable to perform the exhaustive search analysis due to the computing time. Therefore, it is suggested that the number of possible technologies in some subsets be limited to ensure that the total number of possible system is less than a million. Section 6 illustrates an EFD technology selection problem with a smaller number of possible systems, about twenty thousand systems. The sensitivity analysis was successfully conducted with that smaller number of possible systems. One of the future research tasks is to develop optimization methods that can efficiently search the entire (not truncated) solution space using only standard personal computers.

Throughout this section, it is possible to suggest a small number of suitable systems that are particularly attractive for Green Lake drilling site. Six different drilling systems are suggested for this case study as shown in Figure 6-13. Since there are many uncertainties in the inputs being used, decision-makers want to see whether or not small changes in those inputs they use affect the EFD technology selections. Figure 6-15 gives 
an example of the optimal systems of varying the weight on the cost attribute $\left(\mathrm{W}_{1}\right)$ from zero to one. As $\mathrm{W}_{1}$ increases in Figure 6-15, cheaper technologies are selected. For example, the technology selected for subset (1) is changed from conventional diesel trucks to low sulphur diesel trucks with tier III engine and with noise suppressor when $\mathrm{W}_{1}$ increases to 0.6 or more.

Effective displays of sensitivity analyses are crucial as an aid in decision-making process, and also as an aid in explaining EFD technology selections to interested parties. The main purpose of displaying the results of sensitivity analyses graphically is to help the decision-makers better understand what the results mean (Guikema and Milke 2003). Therefore, the display methods chosen in any given situation should be illustrated by the abilities and needs of the decision-makers. For example, more complicated displays such as Figure 6-15 through Figure 6-19 can be used to technically trained people while simpler displays such as Figure 6-20 and Figure 6-29 should be used to less technically trained people.

More extensive sensitivity analyses can be conducted for other input variables such as the utility function for each attribute to suggest more robust optimal systems but they involve a trade-off between increased computational time for the analysis and the potential for increased modeling accuracy. This trade-off needs to be made on a casespecific basis. 


\section{PROTOTYPE OF A WEB-BASED DECISION OPTIMIZATION}

\section{TOOL}

\subsection{Introduction}

A prototype of a web-based application has been developed to help decisionmakers easily follow the systems approach technology evaluation procedure described in Section 5 and then select an optimal drilling system for a specific site. The main reason to develop the web-based application instead of a stand alone computer application is that the qualified users can use the web-based application as long as they can access the Internet regardless of their locations. Furthermore, it can also help to manage used input parameters permanently if a central repository is maintained regularly so that decisionmakers or drilling operators can easily retrieve a previously designed well model for their future operations in different ecosystems.

\subsection{Input and Output Appearance}

The application has been developed by Active Server Pages (ASP) and uses Microsoft Access database. Figure 7-1 shows how to evaluate available technologies within each subset. The left column in the dashed rectangle of Figure 7-1 shows the list of subsets as described in Section 5.2 and the right column of Figure 7-1 shows evaluated technologies (upper) and the input boxes for the evaluation (lower).

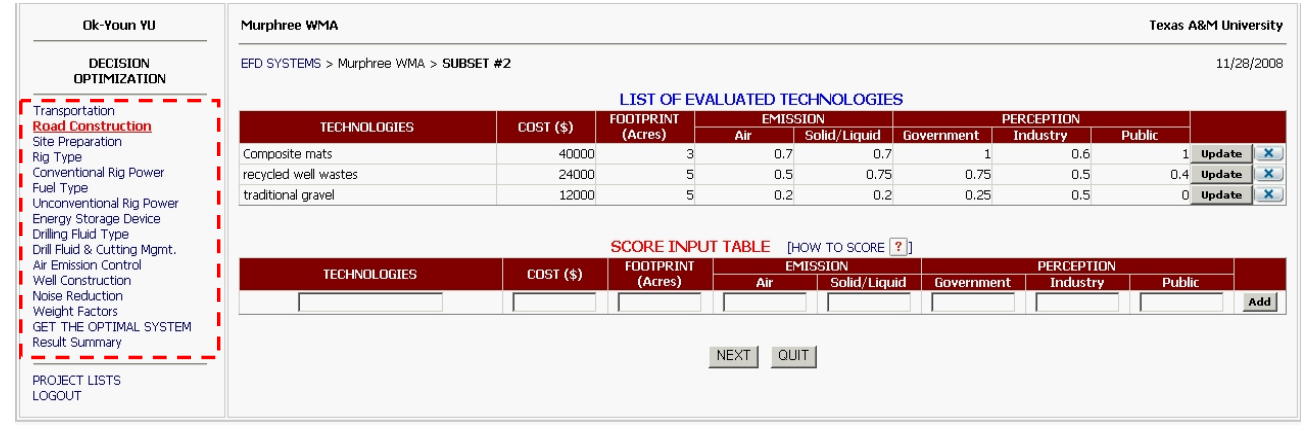

Figure 7-1. Evaluation page for 'Road Construction' subset 
After evaluating available technologies within each subset, users should assign weight factor for each attribute to decide how much important each attribute. All of the weights are positive, and they must sum to one (the standard normalization technique used in decision analysis). The following Figure 7-2 shows an example of assigned two different weight scenarios. Once users assign the weight factors, this application shows up to ten optimal systems (top ten results) for one weight scenario among all possible systems according to users' preference as shown in Figure 7-2.

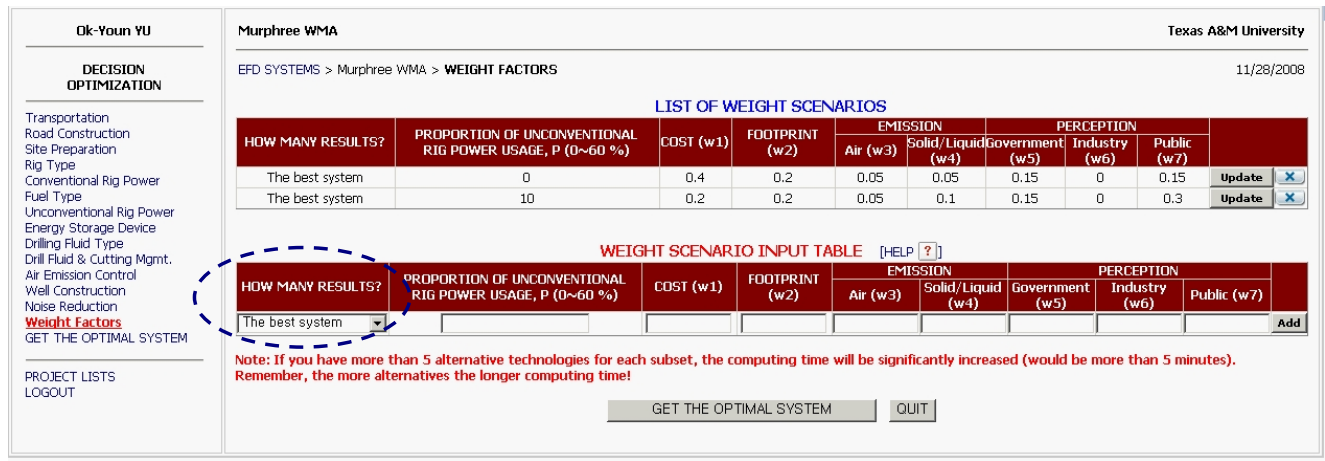

Figure 7-2. Assigned weight scenarios

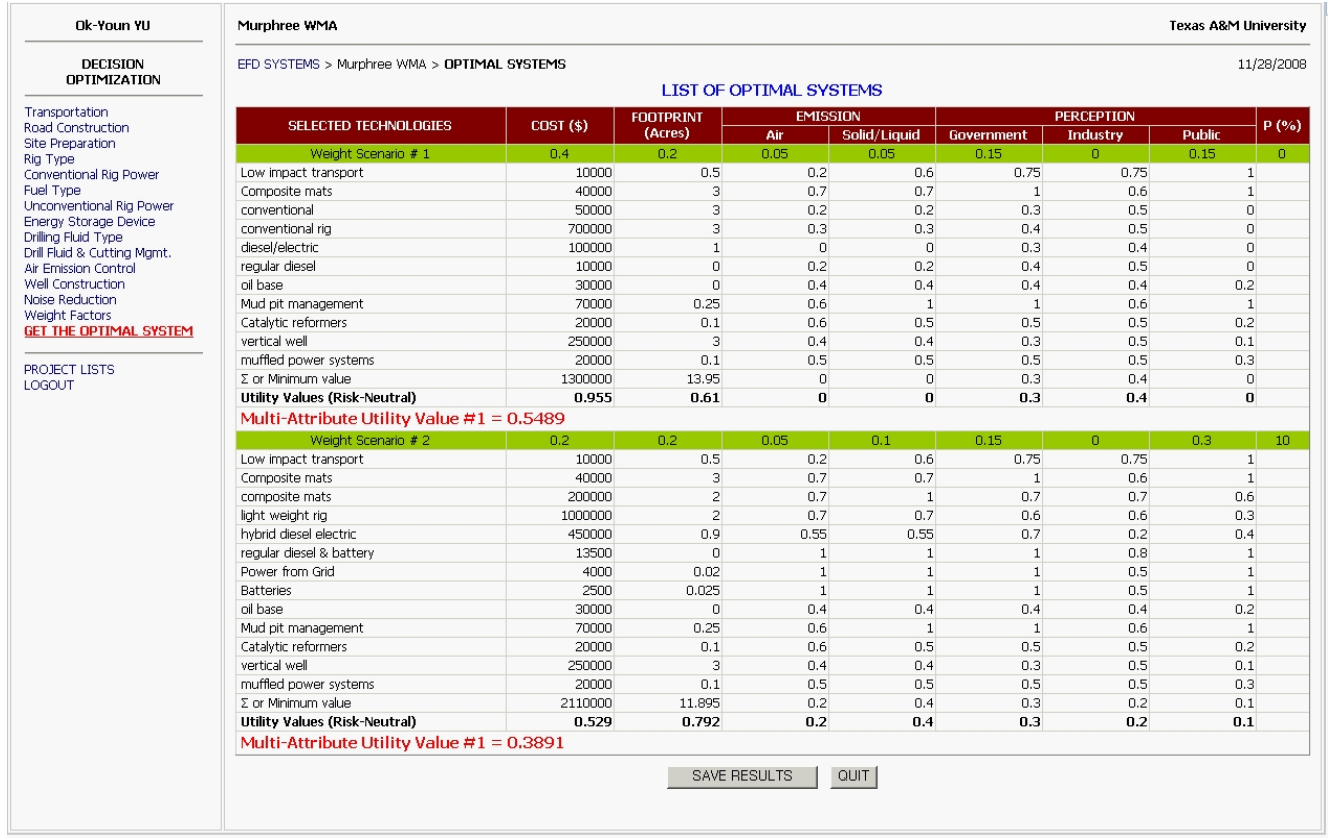

Figure 7-3. An example of the final result page 
Figure 7-3 shows an example of the final result page. In this example, this application shows the technologies selected in the optimal system based on the assigned weight scenario as well as the overall utility score associated with the optimal system. The overall utility score is scaled from zero to one and the higher score is the better.

\subsection{Evaluation of the Web-Base Application}

The Harold Vance Department of Petroleum Engineering at Texas A\&M University has incorporated an EFD system design into its PETE 661 graduate drilling class. The "661 Team Challenge" semester project was assigned to the students to "design a well on paper" using low impact drilling technologies. A systems approach to technology evaluation was utilized to incorporate a number of current and emerging EFD technologies into a single clean drilling system with limited environmental impact.

The Team Challenge project provided a number of positive results to its participants. First, the class members learned first hand of some of the newer technologies available to drilling contractors and operators available for lessening the impact for drilling operations. Next the landowner (McFaddin Ranch personnel) learned of the cost benefit of certain technology some of which could not be justified based on its expense. The exercise provided an excellent "field test" in itself of an EFD optimization system with almost 60 students using the web-based optimization tool to select the most appropriate practices to include in their well designs. Lastly, by having such a large group searching for data, the developer of the software populated the database of technologies with actual cost numbers and contact information. At the conclusion of the study more than 100 different techniques had been identified, characterized, and catalogued.

The answer for the question about the usefulness of the web-based decision optimization tool was an unqualified 'yes' as shown in Figure 7-4. Figure 7-4 is the summary of the responses to a questionnaire distributed at the conclusion of the semester. Almost $74 \%$ of the class students felt that the program helped select optimal systems for their well designs. A compilation of individual responses is contained in APPENDIX C. 


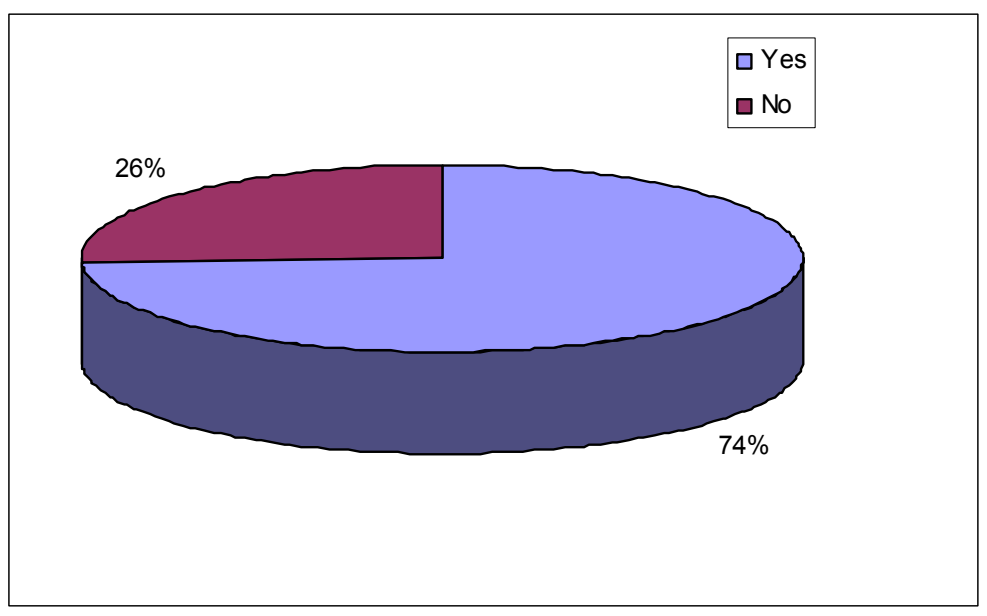

Figure 7-4. Result of survey for the web-based decision optimization tool

It is crucial to keep the web-based application updated as the technology evaluation protocol has been continuously refined by EFD subject matter experts' comments and feedbacks. The list of subsets used in this application, however, is not the same as the one described in Section 5 because the current version of the application has not been updated since January 2008. For example, the technology evaluation protocol described in Section 5 considers 'Cuttings treatment' subset instead of 'Well construction' subset used in the web-based application shown in Figure 7-1. Two other attributes (noise emission and safety) described in Section 5.3 are not also considered in the current web-based application. When estimating the total cost of a technology, drilling time is very important variable because the total cost of the technology should be changed according to the total drilling time of a system. However, it is not also considered in this web-based application yet. One of the future research tasks is to update the current web-based application with the refined technology evaluation protocol. 


\section{SUMMARY AND CONCLUSIONS}

This section reviews the objectives of this research, contribution of this research, and presents the recommendations for future work.

\subsection{Summary}

The key objectives of this research are to:

1. Help the oil industry engineers to get a basic concept about environmentally friendly foundation designs of a rig or an elevated platform for various weights and soil conditions.

2. Develop a technology evaluation protocol based on a systems analysis to synergistically incorporate a number of current and emerging EFD technologies into a single and clean drilling system with limited environmental impact and then to suggest a small number of systems that should be particularly attractive for a given site.

3. Develop a prototype of a web-based decision optimization tool to help decisionmakers easily follow the proposed technology evaluation procedure and then select an optimal drilling system for a specific site.

\subsubsection{Parametric Study of Foundations for Drill Sites}

Three different types of foundations for drill sites were considered in Section 4. First two types were pile foundations (i.e., driven pile and bored pile). About one thousand different cases of pile capacity calculations were conducted depending on various soil types, pile types, and design methods. The optimal pile selection procedure was also described in Section 4.2.4. The other type was Dura-Base Composite Mat. The feasibility study of using the Dura-Base Composite Mat System for the drill site construction was demonstrated with various applied load areas from 6 inches to $10 \mathrm{ft}$ of diameter and soil types. 


\subsubsection{Development of a Systems Approach to Technology Evaluation}

In order to integrate current and new EFD technologies into a viable drilling system compatible with environmentally sensitive areas and finally to suggest a small number of systems that should be particularly attractive for a given site, a quantitative decision tool has been developed based on a systems analysis in Section 5. An optimization scheme is suggested based on a combination of multi-attribute utility theory and exhaustively enumerating all possible technology combinations to provide a quantitative rationale and suggest the best set of systems according to a set of attribute, with the relative importance of the different attribute defined by the decision-maker.

Since an optimal system for a specific site would be based on subjectively assessed data, there can be considerable uncertainty about the input parameters used. Therefore, a sensitivity analysis was conducted using a case study to address this problem in Section 6. The overall procedure is briefly illustrated as follows:

Step 1: Identify the main subsystems, subsets, and technologies within each subset.

Step 2: Define attributes and develop attribute scales to evaluate technologies.

Step 3: Assign scores to all technologies using the attribute scales.

Step 4: For each attribute, calculate the overall attribute score of a system by adding the technology scores or selecting the minimum technology score.

Step 5: For each attribute and in order to homogenize the scores, develop a "utility function $\left(\mathrm{u}_{\mathrm{i}}\right)$ " to convert the overall dimensional score of a system (e.g., \$, acres, and grades) into a non-dimensional utility value (between 0 and 1 ) of the system that reflects the decision-maker(s) value.

Step 6: Decide on a weight factor $\left(\mathrm{k}_{\mathrm{i}}\right)$ for each attribute $\left(\mathrm{i}^{\text {th }}\right)$.

Step 7: Calculate the overall utility score of the system as " $\sum \mathrm{k}_{\mathrm{i}} \mathrm{u}_{\mathrm{i}}$."

Step 8: Use optimization technique to evaluate all possible systems and to find the best system for a specific site.

Step 9: Conduct a sensitivity analysis to examine the impacts of possible changes in the attribute scores and weight factors on the optimal system.

Step 10: Suggest a small number of systems that should be attractive for a given site. 


\subsubsection{A Case Study with Pre-Specified Systems}

An application of the proposed approach was described by conducting a case study in Green Lake at McFaddin, TX. The main purpose of this case study was to test the proposed technology evaluation protocol in a real site and then to refine the protocol. The results of the case study which provided a more logical and comprehensive approach that maximized the economic and environmental goals of both the landowner and the oil company leaseholder were fully described in Section 6.

\subsubsection{Development of a Prototype of a Web-Based Application}

A prototype of a web-based application has been developed to help decisionmakers easily follow the proposed technology evaluation procedure and then select an optimal drilling system for a specific site. The web-based application can also help to manage used input parameters permanently if a central repository is maintained regularly so that decision-makers or drilling operators can easily retrieve a previously designed well model for their future operations in different ecosystems.

\subsection{Conclusions}

Throughout this research, parametric study of foundations for drill sites is conducted, a systems optimization approach is suggested based on a combination of multi-attribute utility theory and exhaustive search optimization, and a web-based decision optimization tool is developed based on the proposed systems approach to technology evaluation. The proposed technology evaluation protocol is designed to help decision-makers with their choices of EFD technology in onshore drilling operations. However, the approach used in this research does have some limitations. The crucial limitation is that the computational burden of the procedure may become prohibitive for problems with a large number of decision variables. One possible way to resolve this problem in this research is if the analyst can identify subsets that will always select the same technology for any weight combinations, the elimination of those subsets from the 
original thirteen subsets can significantly reduce computational burdens in future steps and also simplify the graphical display of sensitivity results.

Since the suggested systems would be based on subjectively assessed data, there can be considerable uncertainty about the input parameters used. In the case study described in Section 6, the most suggested optimal solution (SET 1) is only optimal for about $42 \%$ of the weight combinations tested, which implies that different systems would be suggested for $58 \%$ of plausible weight combinations. It indicates that the sensitivity analysis conducted in Section 6.4 is a worthy topic for further investigation. The sensitivity of the optimal solution to the input parameters and the effects of the uncertainty of those parameters were examined and an approach that can be used to conduct a sensitivity analysis for multi-attribute technology selection problems was presented in Section 6.4. Although the focus of the sensitivity analysis presented in this research has been on sensitivity to weights and overall input attribute scores, the approach could also be applied to sensitivity to risk attitude (i.e., risk-neutral, riskaverse, and risk-seeking) or to other input parameters. The sensitivity to those unapplied input parameters is an important area for further research.

The petroleum industries have several candidate selection methods for some technologies and subsets. For example, they have some common concepts in candidate selection for a managed pressure drilling operation (Rehm et al. 2008). However, these selection methods are only available for a specific technology or subset, not for an entire drilling system.

In conclusion, the technology selection process for a drilling system is mainly based on managerial experience, but a more logical approach based on systems analysis is possible, and additional research could reduce the amount of effort required to use systems analysis for technology selection in a drilling project. Even though the technology selection process can be computationally burdensome, it can be very helpful to decision-makers in refining their decisions on a more scientific basis. 


\subsection{Future Tasks}

In order to encourage petroleum industry people to use environmentally friendly foundations such as elevated platforms and composite mat systems more often for their drilling sites instead of using gravel pads, it is suggested that more specific feasibility study of using those methods including cost estimations be conducted.

Estimating input values for available technologies are a very difficult step to proceed with the quantitative approach suggested in this research. The outcomes of the process should be brought into a question without having the adequate input values. Missing input information introduces additional errors into the analysis because the missing information represents another assumption that must be made to proceed with the analysis (Rehm et al. 2008). One of the future research tasks is to get more EFD subject matter experts' inputs and feedbacks to make the proposed technology selection procedure easier and quicker with more confidence.

Even though exhaustive search optimization used in this research is a simple, practical, and very robust method, it is not recommended for a larger problem due to the computing time. Another future research task is to develop advanced optimization methods that can efficiently search the entire (not truncated) solution space using only standard personal computers. In order to encourage oil industry people to use the proposed technology selection procedure for their real works, it seems to be an essential task.

In Section 6.4, the sensitivity analysis was conducted focused on sensitivity to weights and overall input attribute scores. More extensive sensitivity analyses can be applied to sensitivity to risk attitude (i.e., risk-neutral, risk-averse, and risk-seeking), to the utility function for each attribute, or to other input parameters. The sensitivity to those unapplied input parameters is an important area for further research.

Another future research task is to keep the web-based application developed in this research updated as the technology evaluation protocol has been continuously refined by EFD subject matter experts' comments and feedbacks. This is very important 
task to encourage oil field professionals to try the application for their real works with more confidence.

The single and multi-attribute utility values used in this research represent average estimates that reflect deterministic conditions possibly containing significant and sometimes varying uncertainty components. A new uncertainty-based methodology can be proposed as a future research task for complementing the current technology evaluation protocol by introducing an approach capable of managing properly existing types of evidence (i.e., data, numerical models, and experts' inputs) and their corresponding uncertainty levels. Moreover, it can be proposed to formally account for the causal uncertainty propagation in the common process of a drilling operation, selection of an optimal EFD system, and the assessment of the corresponding environmental impact.

Medina-Cetina et al. (2008) suggested an uncertainty-based system based on causal probability and it will help to identify and quantify major and minor sources of uncertainty, which will propagate towards an uncertainty-based risk index. A risk index will serve as the reference parameter for the selection of an optimal EFD system and will also provide a logical and transparent manner to investigate further key information sources. For instance, two drilling systems with similar overall utility scores may differ significantly in their corresponding uncertainty measures and consequently on the potential losses associated with the selection of one of them. By using a risk index instead, based on default and knowledge building probability distributions (MedinaCetina et al. 2008), it will be possible to differentiate the impact of new evidence or simply the addition of new information into a system so that an optimal drilling system selected for a specific site can be driven not only by weight combinations, but also by the proper uncertainty management leading to the less uncertain system. 


\section{REFERENCES}

ADSC (1999). Drilled Shafts: Construction Procedures and Design Methods, The International Association of Foundation Drilling (ADSC), Dallas, TX.

API RP2A-WSD (2000). American Petroleum Institute Design Code, API, Washington, DC.

API RP2A-LRFD (2003). American Petroleum Institute Design Code, API, Washington, DC.

Briaud, J.-L. (1992). The Pressuremeter, A.A. Balkema, Rotterdam, The Netherlands

Briaud, J. L. (1997). "SALLOP: Simple approach for lateral loads on piles." Journal of Geotechnical and Geoenvironmental Engineering, 123(10), 958.

Call, H., and Merkhofer, M. (1988). "A multi-attribute utility analysis model for ranking superfund sites." Superfund 88: Proceedings of the 9th National Conference, Washington, DC, 44-54.

Clemen, R. T., and Reilly, T. (2001). Making Hard Decisions with DecisionTools, Duxbury, Pacific Grove, CA.

Cover, K. S., Verbunt, J. P. A., de Munck, J. C., and van Dijk, B. W. (2007). "Fitting a single equivalent current dipole model to MEG data with exhaustive search optimization is a simple, practical and very robust method given the speed of modern computers." International Congress Series, 1300, 121-124.

Dyke, K. V. (1997). Fundamentals of Petroleum, Petroleum Extension Service, Austin, TX.

Guikema, S., and Milke, M. (1999). "Quantitative decision tools for conservation programme planning: Practice, theory and potential." Environmental Conservation, 26(03), 179-189.

Guikema, S. D., and Milke, M. W. (2003). "Sensitivity analysis for multi-attribute project selection problems." Civil Engineering and Environmental Systems, 20(3), 143-162. 
Hardaker, J. B. (2004). Coping with Risk in Agriculture, CABI Publishing, Cambridge, MA.

Harrison, W. (2005). "Current environmental practices in petroleum exploration \& production." Energy and the Environment: A Partnership that Works: The Actual Impacts of Oil and Gas Exploration and Development on our Environment, L. C. Gerhard, V. J. Yannacone jr, and C. J. Smith, eds., American Association of Petroleum Geologists, Tulsa, 23.

Hovorka, S. D., Doughty, C., Knox, P. R., Green, C. T., Pruess, K., and Benson, S. M. (2001). "Evaluation of brine-bearing sands of the Frio formation, upper Texas Gulf Coast for geological sequestration of $\mathrm{CO}_{2}$." First National Conference on Carbon Sequestration, Washington, DC, May 14-17.

Jimenez, A., Rios-Insua, S., and Mateos, A. (2003). "A decision support system for multiattribute utility evaluation based on imprecise assignments." Decision Support Systems, 36(1), 65-79.

Kadaster, A. G., and Millheim, K. K. (2004). "Onshore mobile platform: A modular platform for drilling and production operations in remote and environmentally sensitive areas." IADC/SPE Drilling Conference, Dallas, TX, 405-421.

Keeney, R. L. (1974). "Multiplicative utility functions." Operations Research, 22(1), 2234.

Keeney, R. L. (1992). Value-Focused Thinking: A Path to Creative Decisionmaking, Harvard University Press, Cambridge, MA.

Keeney, R. L., and Raiffa, H. (1976). Decisions with Multiple Objectives: Preferences and Value Tradeoffs, Cambridge University Press, New York, NY.

KNR. (1999). Design Criteria for Railroad Bridges, Korean National Railroad (KNR), Republic of Korea.

Medina-Cetina, Z., Feinmberg, J., and Nadim, F. (2008). "Probabilistic Threshold Definition of Early Warning Systems to Natural Hazards." 33rd International Geological Congress, Oslo, Norway, August 6-14. 
Rehm, B., Schubert, J., Haghshenas, A., Paknejad, A., and Hughes, J. (2008). Managed Pressure Drilling, Gulf Publishing Company, Houston, TX.

Rogers, J. D., Knoll, B., Haut, R., McDole, B., and Deskins, G. (2006). "Assessments of Technologies for Environmentally Friendly Drilling Project: Land-Based Operations." Texas A\&M University Environmentally Friendly Drilling report.

RSMeans. (2006). Heavy Construction Cost Data, RS Means Company, Kingston, MA.

\section{Supplemental Sources}

Allett, E. J. (1986). "Environmental impact assessment and decision analysis." The Journal of the Operational Research Society, 37(9), 901-910.

Baecher, G. B., and Christian, J. T. (2003). Reliability and Statistics in Geotechnical Engineering, John Wiley and Sons, Hoboken, NJ.

Butler, J., Morrice, D. J., and Mullarkey, P. W. (2001). "A multiple attribute utility theory approach to ranking and selection." Management Science, 47(6), 800-816.

Corner, J. L., and Kirkwood, C. W. (1991). "Decision analysis applications in the operations research literature, 1970-1989." Operations Research, 39(2), 206-219.

Crawford, D. M., Huntzinger, B. C., and Kirkwood, C. W. (1978). "Multiobjective decision analysis for transmission conductor selection." Management Science, 24(16), 1700-1709.

Freeze, R. A., Massmann, J., Smith, L., Sperling, T., and James, B. (1990). "Hydrogeological decision analysis: 1. A framework." Ground Water, 28(5), 738-766.

Freeze, R. A., James, B., Massmann, J., Sperling, T., and Smith, L. (1992). "Hydrogeological decision analysis: 4. The concept of data worth and its use in the development of site investigation strategies." Ground Water, 30(4), 574-588.

Golab, K., Kirkwood, C. W., and Sicherman, A. (1981). "Selecting a portfolio of solar energy projects using multiattribute preference theory." Management Science, 27(2), 174-189. 
Gray, R. L., and Cambridge, V. J. (1989). "Method for optimization of drilling costs." US Patent 4,845,628.

Guikema, S. D., and Pate-Cornell, M. E. (2002). "Component choice for managing risk in engineered systems with generalized risk/cost functions." Reliability Engineering and System Safety, 78(3), 227-238.

Insua, D. R. (1990). Sensitivity Analysis in Multi-objective Decision Making, SpringerVerlag, New York, NY.

Insua, D. R., and French, S. (1991). "A framework for sensitivity analysis in discrete multi-objective decision-making." European Journal of Operational Research, 54(2), 176-190.

Jonsbraten, T. W. (1998). "Oil field optimization under price uncertainty." The Journal of the Operational Research Society, 49(8), 811-818.

Keeney, R. L., Lathrop, J. F., and Sicherman, A. (1986). "An analysis of Baltimore Gas and Electric Company's technology choice." Operations Research, 34(1), 18-39.

Keeney, R. L., McDaniels, T. L., and Swoveland, C. (1995). "Evaluating improvements in electric utility reliability at British Columbia Hydro." Operations Research, 43(6), 933-947.

Keeney, R. L., and Sicherman, A. (1983). "Illustrative comparison of one utility's coal and nuclear choices." Operations Research, 31(1), 50-83.

Keeney, R. L., and Winterfeldt, D. (1994). "Managing nuclear waste from power plants." Risk Analysis, 14(1), 107-130.

Kim, J., Hobbs, B. F., and Koonce, J. F. (2007). "Analysis of the sensitivity of decision analysis results to errors and simplifications in problem structure: Application to Lake Erie Ecosystem Management." IEEE Transactions on Systems, Man and Cybernetics, Part A, 37(4), 505-518.

Martello, S., and Toth, P. (1990). Knapsack Problems: Algorithms and Computer Implementations, John Wiley \& Sons, New York, NY. 
Massmann, J., Freeze, R. A., Smith, L., Sperling, T., and James, B. (1991). "Hydrogeological decision analysis: 2. Applications to ground-water contamination." Ground Water, 29(4), 536-548.

Mateos, A., Rios-Insua, S., and Gallego, E. (2001). "Postoptimal analysis in a multiattribute decision model for restoring contaminated aquatic ecosystems." The Journal of the Operational Research Society, 52(7), 727-738.

North, D. W., and Stengel, D. N. (1982). "Decision analysis of program choices in magnetic fusion energy development." Management Science, 28(3), 276-288.

Onwubolu, G. C., and Clerc, M. (2004). "Optimal path for automated drilling operations by a new heuristic approach using particle swarm optimization." International Journal of Production Research, 42(3), 473-491.

Proll, L. G., Insua, D. R., and Salhi, A. (1993). "Mathematical programming and the sensitivity of multi-criteria decisions." Annals of Operations Research, 43(1-4), 109-122.

Reinhold, W. B., and Close, D. A. "Drilling optimization--The driller's role." SPE Drilling \& Completion1997, 12(1), 5-12.

Reynolds, W. W. (1986). "Economic analysis of drilling plans and contractors by use of a drilling systems approach." J. Pet. Technol, 38(8).

Salkin, H. M., and de Kluyver, C. A. (1975). "The knapsack problem: a survey." Naval Research Logistics Quarterly, 22(1), 127-44.

Shachter, R. D. (1986). "Evaluating influence diagrams." Operations Research, 34(6), 871-882.

Sperling, T., Freeze, R. A., Massmann, J., Smith, L., and James, B. (1992). "Hydrogeological decision analysis: 3. Application to design of a ground-water control system at an open pit mine." Ground Water, 30(3), 376-389.

Triantaphyllou, E., and Sanchez, A. (1997). "A sensitivity analysis approach for some deterministic multi-criteria decision-making methods." Decision Sciences, 28(1), 151-194. 


\section{APPENDIX A}

\section{PILE FOUNDATION DESIGNS}




\section{$\square$ Design Condition}

Unfactored vertical load $s=1000.000$ kips

$65 \%$ of the vertical loads were distributed across 6 modules,

$\therefore \frac{1000.000 \times 0.650}{6.000}=108.333$ kips (per module)

Unfactored dead load $(30 \%$ of the total vertical loads $)=\quad 32.500 \mathrm{kips} \quad$ (per module)

Unfactored live load ( $70 \%$ of the total vertical loads) $=75.833 \mathrm{kips} \quad$ (per module)

Module Layout

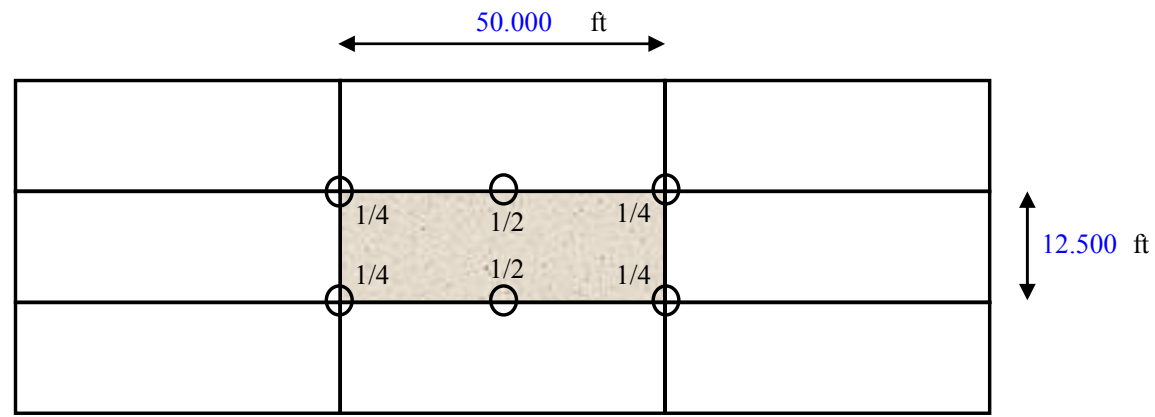

Wind Force Calculations (API RP2A - LRFD)

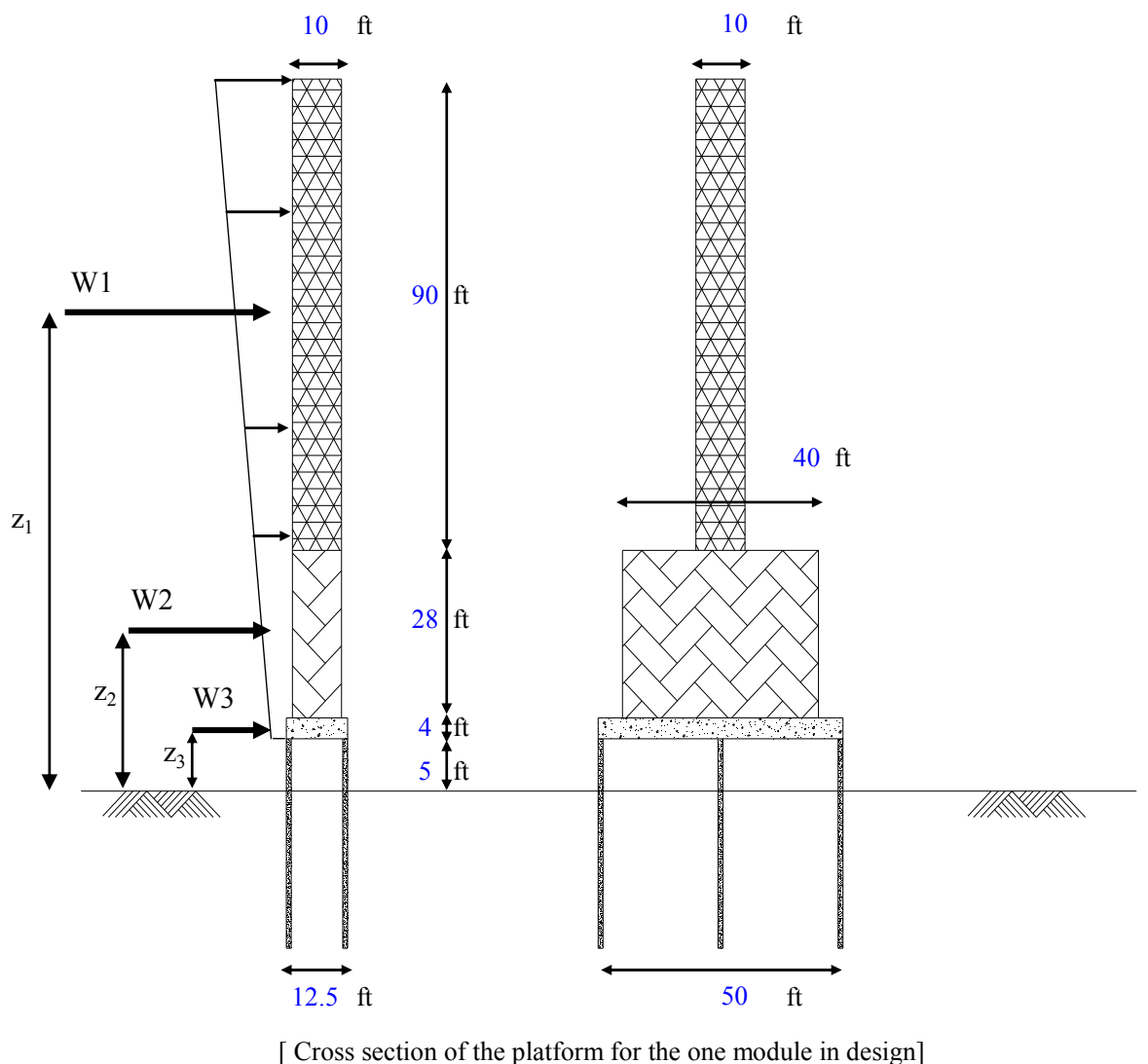




$\begin{array}{rllllllll}\mathrm{z}_{1} & =82 \mathrm{ft} & \mathrm{A} 1 & = & 900.000 & \mathrm{ft}^{2} & \text { Normal V } & =25 \mathrm{~m} / \mathrm{s} \\ \mathrm{z}_{2} & =23 \mathrm{ft} & \mathrm{A} 2 & = & 1120.000 & \mathrm{ft}^{2} & \text { Extreme V } & =49 \mathrm{~m} / \mathrm{s} \\ \mathrm{z}_{3} & = & 7 \mathrm{ft} & \mathrm{A} 3= & 200.000 & \mathrm{ft}^{2} & \mathrm{Cs} & =1.0 \\ \rho & =0.00238 \mathrm{lb} \cdot \mathrm{sec}^{2} / \mathrm{ft}^{4} & & & & & \end{array}$

For the $\mathrm{W} 1$ (distance from the top of the pile $=77 \mathrm{ft}$ ),

$$
\begin{aligned}
\mathrm{V} 1=\mathrm{V}\left(1 \mathrm{hr}, \mathrm{z}_{\mathrm{R}}\right)\left(\mathrm{z}_{1} / \mathrm{z}_{\mathrm{R}}\right)^{0.125}=(25 \mathrm{~m} / \mathrm{sec})(3.3 \mathrm{ft} / \mathrm{sec})(82 / 33)^{0.125}=92.441 \mathrm{ft} / \mathrm{sec} \\
\mathrm{W} 1=(\rho / 2)(\mathrm{V} 1)^{2} \mathrm{CsA}=(0.00238 / 2)(92.441)^{2}(1)(900)=9.152 \mathrm{kips}
\end{aligned}
$$

For the extreme We1,

$$
\begin{aligned}
& \mathrm{Ve1}=\mathrm{V}\left(1 \mathrm{hr}, \mathrm{z}_{\mathrm{R}}\right)\left(\mathrm{z}_{1} / \mathrm{z}_{\mathrm{R}}\right)^{0.125}=(49 \mathrm{~m} / \mathrm{sec})(3.3 \mathrm{ft} / \mathrm{sec})(82 / 33)^{0.125}=181.185 \mathrm{ft} / \mathrm{sec} \\
& \mathrm{We1}=(\mathrm{\rho} / 2)(\mathrm{Ve} 1)^{2} \mathrm{CsA}=(0.00238 / 2)(181.185)^{2}(1)(900)=\mathbf{3 5 . 1 5 9} \mathrm{kips}
\end{aligned}
$$

For the W2 (distance from the top of the pile $=18 \mathrm{ft}$ ),

$$
\begin{aligned}
& \mathrm{V} 2=\mathrm{V}\left(1 \mathrm{hr}, \mathrm{z}_{\mathrm{R}}\right)\left(\mathrm{z}_{2} / \mathrm{z}_{\mathrm{R}}\right)^{0.125}=(25 \mathrm{~m} / \mathrm{sec})(3.3 \mathrm{ft} / \mathrm{sec})(23 / 33)^{0.125}=78.860 \mathrm{ft} / \mathrm{sec} \\
& \mathrm{W} 2=(\rho / 2)(\mathrm{V} 2)^{2} \mathrm{CsA}=(0.00238 / 2)(78.86)^{2}(1)(1120)=\mathbf{8 . 2 8 9} \mathrm{kips}
\end{aligned}
$$

For the extreme We2,

$$
\begin{aligned}
& \mathrm{Ve} 2=\mathrm{V}\left(1 \mathrm{hr}, \mathrm{z}_{\mathrm{R}}\right)\left(\mathrm{z}_{2} / \mathrm{z}_{\mathrm{R}}\right)^{0.125}=(49 \mathrm{~m} / \mathrm{sec})(3.3 \mathrm{ft} / \mathrm{sec})(23 / 33)^{0.125}=154.565 \mathrm{ft} / \mathrm{sec} \\
& \mathrm{We}=(\mathrm{\rho} / 2)(\mathrm{Ve} 2)^{2} \mathrm{CsA}=(0.00238 / 2)(154.565)^{2}(1)(1120)=\mathbf{3 1 . 8 4 1} \mathrm{kips}
\end{aligned}
$$

For the W3 (distance from the top of the pile $=2 \mathrm{ft}$ ),

$$
\begin{aligned}
& \left.\mathrm{V} 3=\mathrm{V}\left(1 \mathrm{hr}, \mathrm{z}_{\mathrm{R}}\right)\left(\mathrm{z}_{3} / \mathrm{z}_{\mathrm{R}}\right)^{0.125}=(25 \mathrm{~m} / \mathrm{sec})(3.3 \mathrm{ft} / \mathrm{sec})(7 / 33)\right)^{0.125}=67.964 \mathrm{ft} / \mathrm{sec} \\
& \mathrm{W} 3=(\rho / 2)(\mathrm{V} 3)^{2} \mathrm{CsA}=(0.00238 / 2)(67.964)^{2}(1)(200)=\mathbf{1 . 0 9 9} \mathrm{kips}
\end{aligned}
$$

For the extreme We3,

$$
\begin{aligned}
& \mathrm{Ve3}=\mathrm{V}\left(1 \mathrm{hr}, \mathrm{z}_{\mathrm{R}}\right)\left(\mathrm{z}_{3} / \mathrm{z}_{\mathrm{R}}\right)^{0.125}=(49 \mathrm{~m} / \mathrm{sec})(3.3 \mathrm{ft} / \mathrm{sec})(7 / 33)^{0.125}=133.209 \mathrm{ft} / \mathrm{sec} \\
& \mathrm{We3}=(\rho / 2)(\mathrm{Ve} 3)^{2} \mathrm{CsA}=(0.00238 / 2)(133.209)^{2}(1)(200)=\mathbf{4 . 2 2 3} \mathrm{kips}
\end{aligned}
$$




\section{$\square$ Load Combinations (API RP2A - LRFD)}
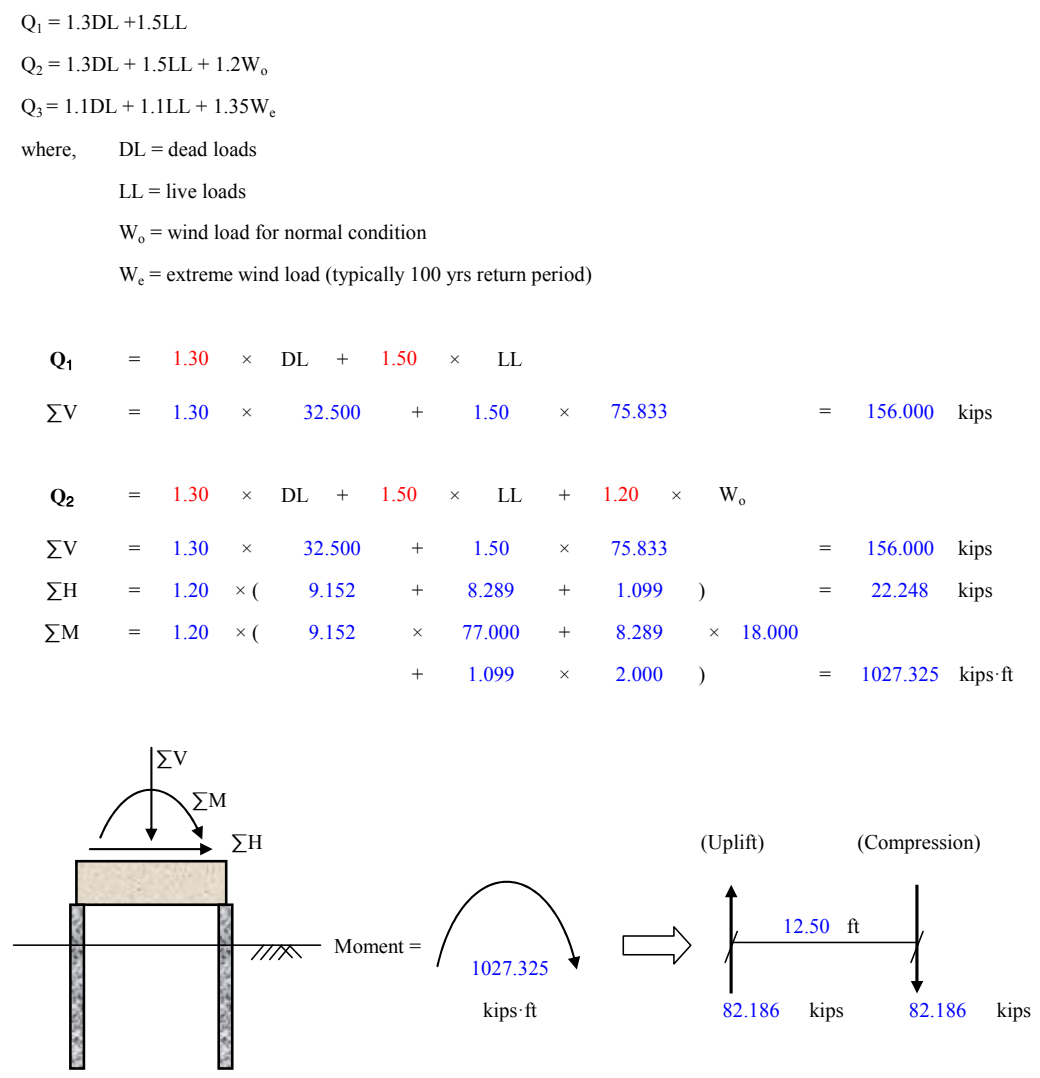

Hence,

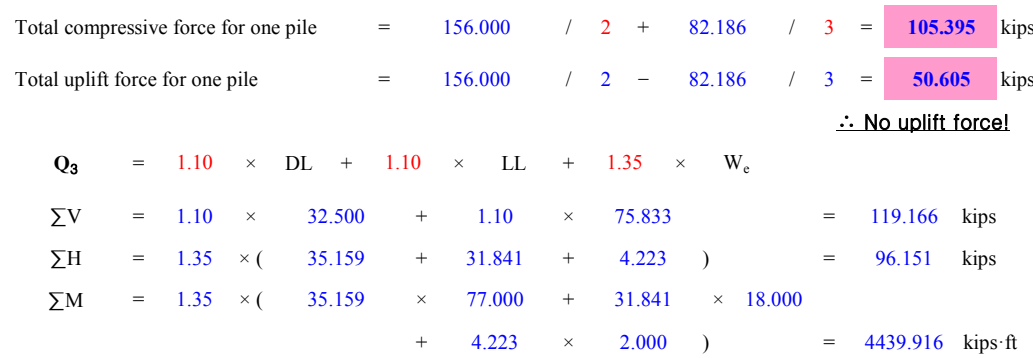

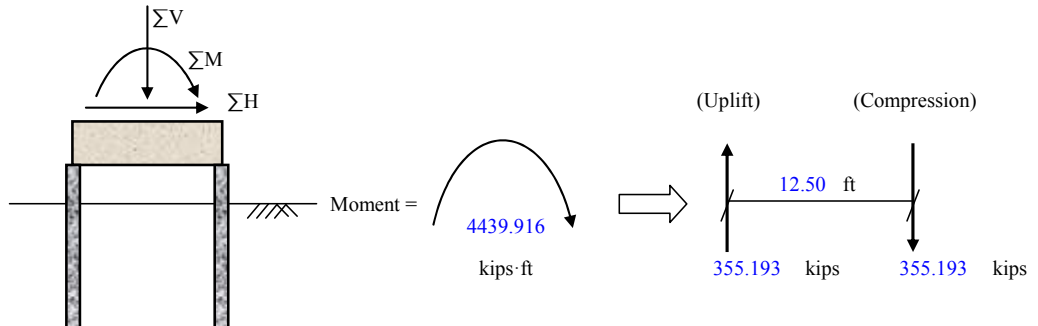

Hence,

Total compressive force for one pile $=119.166 / 2+355.193 / 3=\mathbf{1 7 7 . 9 8 1}$ kips Total uplift force for one pile $\quad=\quad 119.166 / 2-355.193 / 3=\mathbf{- 5 8 . 8 1 5}$ kips 
$\square$ Soil Condition

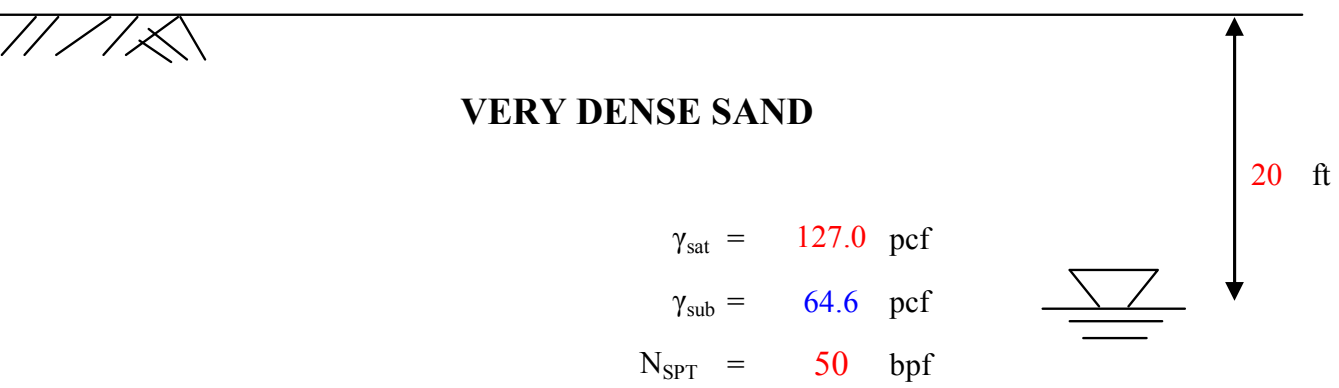

\section{Vertical Capacity of the Pile (RP2A)}

a. Unit end bearing capacity

$\mathrm{q}_{\mathrm{p}}=\mathrm{P}_{\mathrm{o}}{ }^{\prime} \cdot \mathrm{N}_{\mathrm{q}}$

where: $\quad \mathrm{P}_{\mathrm{o}}{ }^{\prime}=$ effective overburden pressure at the point in question

$\mathrm{N}_{\mathrm{q}}=$ dimensionless bearing capacity factor $=50$ (very dense)

b. Unit skin friction capacity

$\mathrm{fs} \quad=\mathrm{K} \cdot \mathrm{P}_{\mathrm{o}}{ }^{\prime} \cdot \tan \delta$

where: $\mathrm{K}=$ dimensionless coefficient of lateral earth pressure (ratio of horizontal to vertical normal effective stress)

$=1.00$ (full displacement piles, plugged or closed end)

$\delta=$ friction angle between the soil and pile wall $=35^{\circ}$ (very dense)

c. Ultimate vertical capacity

$\mathrm{Qu}=\mathrm{Qp}+\mathrm{Qs} \quad=\mathrm{q}_{\mathrm{p}} \mathrm{Ap}+\mathrm{fs} A \mathrm{~s}$

where: $\quad \mathrm{Ap}=$ gross end area of pile $=\pi \mathrm{D}^{2} / 4$ (where, $\mathrm{D}$ : diameter of pile)

As $=$ side surface area of pile $=\pi D L$ (where, $L$ : length of pile) 
1. Operating Environmental Conditions

Resistance Factors, $\varphi=\quad 0.7$

Diameter, $D=12$ inches

\begin{tabular}{|c|c|c|c|c|c|c|c|c|}
\hline Depth [ft] & $\mathrm{P}_{\mathrm{o}}{ }^{\prime}[\mathrm{psf}]$ & $\mathrm{q}_{\mathrm{p}}[\mathrm{ksf}]$ & $\mathrm{Qp}[\mathrm{kips}]$ & $\mathrm{fs}[\mathrm{ksf}]$ & $\Sigma \mathrm{Qs}[\mathrm{kips}]$ & $\mathrm{Qu}[\mathrm{kips}]$ & $\varphi \mathrm{Qu}[\mathrm{kips}]$ & $\varphi \mathrm{Qu}($ uplift) \\
\hline 0 & 0 & 0 & 0 & 0 & 0 & 0 & 0 & 0 \\
\hline 5 & 635.000 & 31.750 & 24.936 & 0.222 & 3.487 & 28.423 & 19.896 & 2.441 \\
\hline 10 & 1270.000 & 63.500 & 49.873 & 0.445 & 13.980 & 63.853 & 44.697 & 9.786 \\
\hline 15 & 1905.000 & 95.250 & 74.809 & 0.667 & 31.432 & 106.241 & 74.369 & 22.002 \\
\hline 20 & 2540.000 & 127.000 & 99.746 & 0.889 & 55.858 & 155.604 & 108.923 & 39.101 \\
\hline 25 & 2863.000 & 143.150 & 112.430 & 1.090 & 85.577 & 198.007 & 138.605 & 59.904 \\
\hline 30 & 3186.000 & 159.300 & 125.114 & 1.261 & 118.846 & 243.960 & 170.772 & 83.192 \\
\hline 35 & 3509.000 & 175.450 & 137.798 & 1.414 & 155.531 & 293.329 & 205.330 & 108.872 \\
\hline 40 & 3832.000 & 191.600 & 150.482 & 1.538 & 193.230 & 343.712 & 240.598 & 135.261 \\
\hline
\end{tabular}

Diameter, $D=16$ inches

\begin{tabular}{|c|c|c|c|c|c|c|c|c|}
\hline Depth [ft] & $\mathrm{P}_{\mathrm{o}}{ }^{\prime}[\mathrm{psf}]$ & $\mathrm{q}_{\mathrm{p}}[\mathrm{ksf}]$ & Qp [kips] & fs [ksf] & $\Sigma \mathrm{Qs}[\mathrm{kips}]$ & Qu [kips] & $\varphi \mathrm{Qu}[\mathrm{kips}]$ & $\varphi \mathrm{Qu}($ uplift) \\
\hline 0 & 0 & 0 & 0 & 0 & 0 & 0 & 0 & 0 \\
\hline 5 & 635.000 & 31.750 & 44.331 & 0.222 & 4.650 & 48.981 & 34.287 & 3.255 \\
\hline 10 & 1270.000 & 63.500 & 88.663 & 0.445 & 18.640 & 107.303 & 75.112 & 13.048 \\
\hline 15 & 1905.000 & 95.250 & 132.994 & 0.667 & 41.909 & 174.903 & 122.432 & 29.336 \\
\hline 20 & 2540.000 & 127.000 & 177.325 & 0.889 & 74.477 & 251.802 & 176.261 & 52.134 \\
\hline 25 & 2863.000 & 143.150 & 199.875 & 1.090 & 114.103 & 313.978 & 219.785 & 79.872 \\
\hline 30 & 3186.000 & 159.300 & 222.425 & 1.261 & 158.462 & 380.887 & 266.621 & 110.923 \\
\hline 35 & 3509.000 & 175.450 & 244.974 & 1.414 & 207.375 & 452.349 & 316.644 & 145.163 \\
\hline 40 & 3832.000 & 191.600 & 267.524 & 1.538 & 257.641 & 525.165 & 367.616 & 180.349 \\
\hline
\end{tabular}

Diameter, $D=20$ inches

\begin{tabular}{|c|c|c|c|c|c|c|c|c|}
\hline Depth [ft] & $\mathrm{P}_{\mathrm{o}}{ }^{\prime}[\mathrm{psf}]$ & $\mathrm{q}_{\mathrm{p}}[\mathrm{ksf}]$ & Qp [kips] & $\mathrm{fs}[\mathrm{ksf}]$ & $\Sigma \mathrm{Qs}[\mathrm{kips}]$ & $\mathrm{Qu}[\mathrm{kips}]$ & $\varphi \mathrm{Qu}[\mathrm{kips}]$ & $\varphi \mathrm{Qu}($ uplift) \\
\hline 0 & 0 & 0 & 0 & 0 & 0 & 0 & 0 & 0 \\
\hline 5 & 635.000 & 31.750 & 69.268 & 0.222 & 5.812 & 75.080 & 52.556 & 4.068 \\
\hline 10 & 1270.000 & 63.500 & 138.536 & 0.445 & 23.300 & 161.836 & 113.285 & 16.310 \\
\hline 15 & 1905.000 & 95.250 & 207.803 & 0.667 & 52.386 & 260.189 & 182.132 & 36.670 \\
\hline 20 & 2540.000 & 127.000 & 277.071 & 0.889 & 93.096 & 370.167 & 259.117 & 65.167 \\
\hline 25 & 2863.000 & 143.150 & 312.305 & 1.090 & 142.628 & 454.933 & 318.453 & 99.840 \\
\hline 30 & 3186.000 & 159.300 & 347.539 & 1.261 & 198.077 & 545.616 & 381.931 & 138.654 \\
\hline 35 & 3509.000 & 175.450 & 382.773 & 1.414 & 259.219 & 641.992 & 449.394 & 181.453 \\
\hline 40 & 3832.000 & 191.600 & 418.006 & 1.538 & 322.051 & 740.057 & 518.040 & 225.436 \\
\hline
\end{tabular}

Diameter, $D=24$ inches

\begin{tabular}{|c|c|c|c|c|c|c|c|c|}
\hline Depth [ft] & $\mathrm{P}_{\mathrm{o}}{ }^{\prime}[\mathrm{psf}]$ & $\mathrm{q}_{\mathrm{p}}[\mathrm{ksf}]$ & $\mathrm{Qp}[\mathrm{kips}]$ & $\mathrm{fs}[\mathrm{ksf}]$ & $\Sigma \mathrm{Qs}[\mathrm{kips}]$ & $\mathrm{Qu}[\mathrm{kips}]$ & $\varphi \mathrm{Qu}[\mathrm{kips}]$ & $\varphi \mathrm{Qu}($ uplift) \\
\hline 0 & 0 & 0 & 0 & 0 & 0 & 0 & 0 & 0 \\
\hline 5 & 635.000 & 31.750 & 99.746 & 0.222 & 6.974 & 106.720 & 74.704 & 4.882 \\
\hline 10 & 1270.000 & 63.500 & 199.491 & 0.445 & 27.960 & 227.451 & 159.216 & 19.572 \\
\hline 15 & 1905.000 & 95.250 & 299.237 & 0.667 & 62.863 & 362.100 & 253.470 & 44.004 \\
\hline 20 & 2540.000 & 127.000 & 398.982 & 0.889 & 111.715 & 510.697 & 357.488 & 78.201 \\
\hline 25 & 2863.000 & 143.150 & 449.719 & 1.090 & 171.154 & 620.873 & 434.611 & 119.808 \\
\hline 30 & 3186.000 & 159.300 & 500.456 & 1.261 & 237.693 & 738.149 & 516.704 & 166.385 \\
\hline 35 & 3509.000 & 175.450 & 551.192 & 1.414 & 311.063 & 862.255 & 603.579 & 217.744 \\
\hline 40 & 3832.000 & 191.600 & 601.929 & 1.538 & 386.461 & 988.390 & 691.873 & 270.523 \\
\hline
\end{tabular}

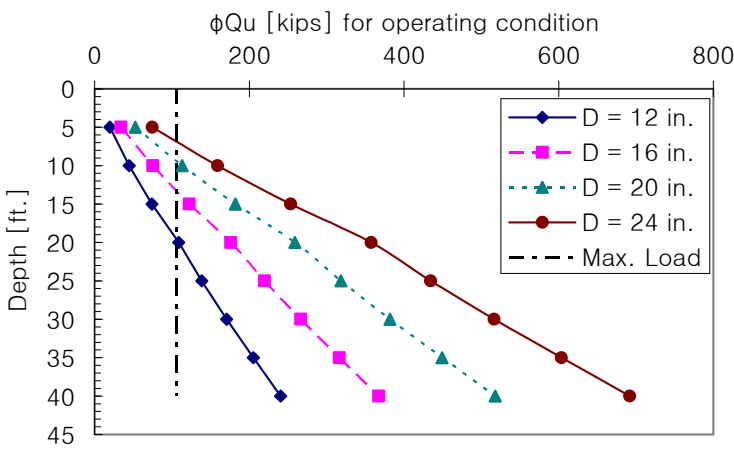


2. Extreme Environmental Conditions

Resistance Factors, $\varphi=$

0.8

0.7 (uplift)

Diameter, $D=12$ inches

\begin{tabular}{|c|c|c|c|c|c|c|c|c|}
\hline Depth [ft] & $\mathrm{P}_{\mathrm{o}}{ }^{\prime}[\mathrm{psf}]$ & $\mathrm{q}_{\mathrm{p}}[\mathrm{ksf}]$ & Qp [kips] & $\mathrm{fs}[\mathrm{ksf}]$ & $\Sigma \mathrm{Qs}[\mathrm{kips}]$ & $\mathrm{Qu}[\mathrm{kips}]$ & $\varphi \mathrm{Qu}[\mathrm{kips}]$ & $\varphi \mathrm{Qu}(\mathrm{uplift})$ \\
\hline 0 & 0 & 0 & 0 & 0 & 0 & 0 & 0 & 0 \\
\hline 5 & 635.000 & 31.750 & 24.936 & 0.222 & 3.487 & 28.423 & 22.738 & 2.441 \\
\hline 10 & 1270.000 & 63.500 & 49.873 & 0.445 & 13.980 & 63.853 & 51.082 & 9.786 \\
\hline 15 & 1905.000 & 95.250 & 74.809 & 0.667 & 31.432 & 106.241 & 84.993 & 22.002 \\
\hline 20 & 2540.000 & 127.000 & 99.746 & 0.889 & 55.858 & 155.604 & 124.483 & 39.101 \\
\hline 25 & 2863.000 & 143.150 & 112.430 & 1.090 & 85.577 & 198.007 & 158.406 & 59.904 \\
\hline 30 & 3186.000 & 159.300 & 125.114 & 1.261 & 118.846 & 243.960 & 195.168 & 83.192 \\
\hline 35 & 3509.000 & 175.450 & 137.798 & 1.414 & 155.531 & 293.329 & 234.663 & 108.872 \\
\hline 40 & 3832.000 & 191.600 & 150.482 & 1.538 & 193.230 & 343.712 & 274.970 & 135.261 \\
\hline
\end{tabular}

Diameter, $D=16$ inches

\begin{tabular}{|c|c|c|c|c|c|c|c|c|}
\hline Depth [ft] & $\mathrm{P}_{\mathrm{o}}{ }^{\prime}[\mathrm{psf}]$ & $\mathrm{q}_{\mathrm{p}}[\mathrm{ksf}]$ & Qp [kips] & $\mathrm{fs}[\mathrm{ksf}]$ & $\Sigma \mathrm{Qs}[\mathrm{kips}]$ & Qu [kips] & $\varphi \mathrm{Qu}[\mathrm{kips}]$ & $\varphi \mathrm{Qu}($ uplift) \\
\hline 0 & 0 & 0 & 0 & 0 & 0 & 0 & 0 & 0 \\
\hline 5 & 635.000 & 31.750 & 44.331 & 0.222 & 4.650 & 48.981 & 39.185 & 3.255 \\
\hline 10 & 1270.000 & 63.500 & 88.663 & 0.445 & 18.640 & 107.303 & 85.842 & 13.048 \\
\hline 15 & 1905.000 & 95.250 & 132.994 & 0.667 & 41.909 & 174.903 & 139.922 & 29.336 \\
\hline 20 & 2540.000 & 127.000 & 177.325 & 0.889 & 74.477 & 251.802 & 201.442 & 52.134 \\
\hline 25 & 2863.000 & 143.150 & 199.875 & 1.090 & 114.103 & 313.978 & 251.182 & 79.872 \\
\hline 30 & 3186.000 & 159.300 & 222.425 & 1.261 & 158.462 & 380.887 & 304.710 & 110.923 \\
\hline 35 & 3509.000 & 175.450 & 244.974 & 1.414 & 207.375 & 452.349 & 361.879 & 145.163 \\
\hline 40 & 3832.000 & 191.600 & 267.524 & 1.538 & 257.641 & 525.165 & 420.132 & 180.349 \\
\hline
\end{tabular}

Diameter, $D=20$ inches

\begin{tabular}{|c|c|c|c|c|c|c|c|c|}
\hline Depth [ft] & $\mathrm{P}_{\mathrm{o}}{ }^{\prime}[\mathrm{psf}]$ & $\mathrm{q}_{\mathrm{p}}[\mathrm{ksf}]$ & $\mathrm{Qp}[\mathrm{kips}]$ & $\mathrm{fs}[\mathrm{ksf}]$ & $\Sigma \mathrm{Qs}[\mathrm{kips}]$ & Qu [kips] & $\varphi \mathrm{Qu}[\mathrm{kips}]$ & $\varphi \mathrm{Qu}$ (uplift) \\
\hline 0 & 0 & 0 & 0 & 0 & 0 & 0 & 0 & 0 \\
\hline 5 & 635.000 & 31.750 & 69.268 & 0.222 & 5.812 & 75.080 & 60.064 & 4.068 \\
\hline 10 & 1270.000 & 63.500 & 138.536 & 0.445 & 23.300 & 161.836 & 129.469 & 16.310 \\
\hline 15 & 1905.000 & 95.250 & 207.803 & 0.667 & 52.386 & 260.189 & 208.151 & 36.670 \\
\hline 20 & 2540.000 & 127.000 & 277.071 & 0.889 & 93.096 & 370.167 & 296.134 & 65.167 \\
\hline 25 & 2863.000 & 143.150 & 312.305 & 1.090 & 142.628 & 454.933 & 363.946 & 99.840 \\
\hline 30 & 3186.000 & 159.300 & 347.539 & 1.261 & 198.077 & 545.616 & 436.493 & 138.654 \\
\hline 35 & 3509.000 & 175.450 & 382.773 & 1.414 & 259.219 & 641.992 & 513.594 & 181.453 \\
\hline 40 & 3832.000 & 191.600 & 418.006 & 1.538 & 322.051 & 740.057 & 592.046 & 225.436 \\
\hline
\end{tabular}

Diameter, $D=24$ inches

\begin{tabular}{|c|c|c|c|c|c|c|c|c|}
\hline Depth [ft] & $\mathrm{P}_{\mathrm{o}}{ }^{\prime}[\mathrm{psf}]$ & $\mathrm{q}_{\mathrm{p}}[\mathrm{ksf}]$ & $\mathrm{Qp}[\mathrm{kips}]$ & $\mathrm{fs}[\mathrm{ksf}]$ & $\Sigma \mathrm{Qs}[\mathrm{kips}]$ & $\mathrm{Qu}[\mathrm{kips}]$ & $\varphi \mathrm{Qu}[\mathrm{kips}]$ & $\varphi \mathrm{Qu}(\mathrm{uplift})$ \\
\hline 0 & 0 & 0 & 0 & 0 & 0 & 0 & 0 & 0 \\
\hline 5 & 635.000 & 31.750 & 99.746 & 0.222 & 6.974 & 106.720 & 85.376 & 4.882 \\
\hline 10 & 1270.000 & 63.500 & 199.491 & 0.445 & 27.960 & 227.451 & 181.961 & 19.572 \\
\hline 15 & 1905.000 & 95.250 & 299.237 & 0.667 & 62.863 & 362.100 & 289.680 & 44.004 \\
\hline 20 & 2540.000 & 127.000 & 398.982 & 0.889 & 111.715 & 510.697 & 408.558 & 78.201 \\
\hline 25 & 2863.000 & 143.150 & 449.719 & 1.090 & 171.154 & 620.873 & 496.698 & 119.808 \\
\hline 30 & 3186.000 & 159.300 & 500.456 & 1.261 & 237.693 & 738.149 & 590.519 & 166.385 \\
\hline 35 & 3509.000 & 175.450 & 551.192 & 1.414 & 311.063 & 862.255 & 689.804 & 217.744 \\
\hline 40 & 3832.000 & 191.600 & 601.929 & 1.538 & 386.461 & 988.390 & 790.712 & 270.523 \\
\hline
\end{tabular}
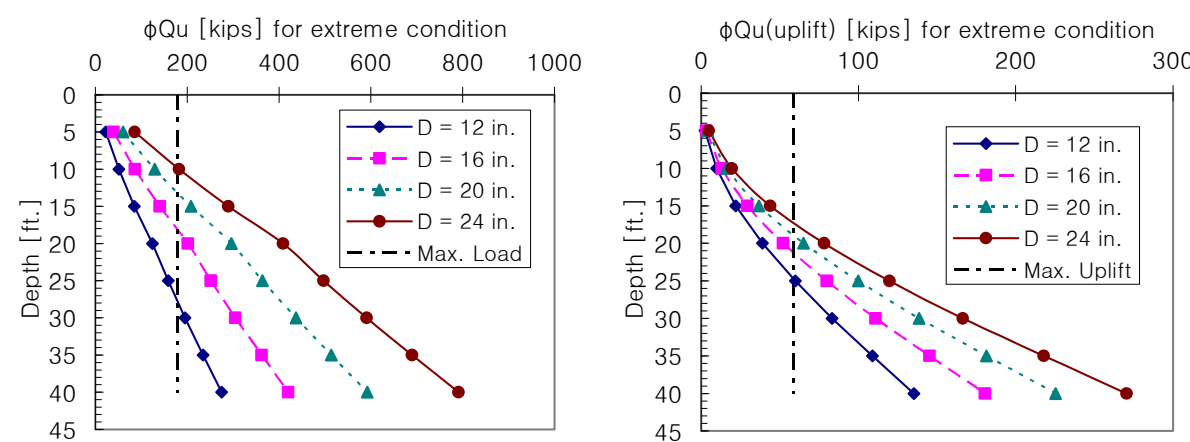
$\square$ Horizontal Capacity of the Pile (SALLOP)

For free head,

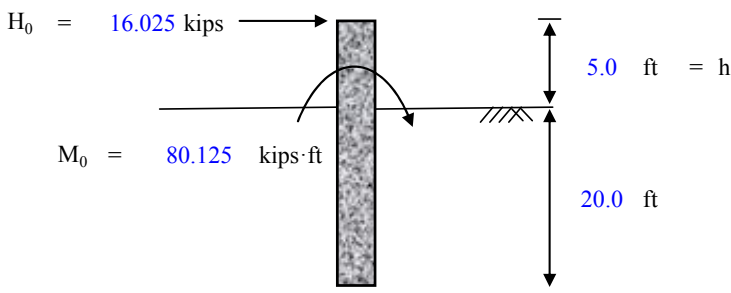

20 in. (wall thickness $=0.375$ in.)

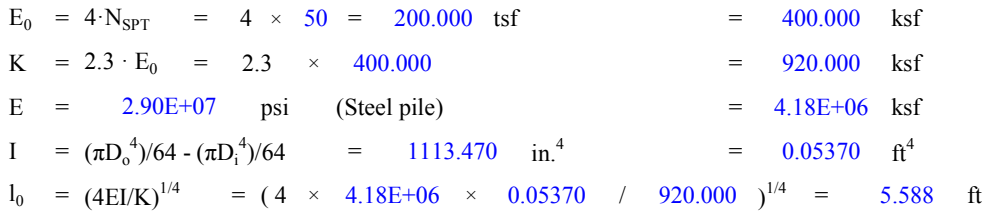

where, $\mathrm{E}_{0} \quad=$ pressuremeter load modulus

$\mathrm{K}=$ soil spring constant

$\mathrm{E}=$ modulus of elasticity for the pile material

$\mathrm{I}=$ moment of inertia for the pile, $\mathrm{H}_{0}=$ horizontal load

$1_{0}=$ transfer length $\quad, \mathrm{M}_{0}=$ applied moment at the ground surface

Embedded pile length, $\mathrm{L}=20.0 \mathrm{ft} \geq 3 \cdot 1_{\circ}=16.764 \mathrm{ft} \quad \therefore$ long flexible pile!

\section{Lateral Pile Capacity}

The zero-shear depth $\mathrm{D}_{\mathrm{v}}$ is obtained by setting $\mathrm{V}$ (shear force) $=0$;

$\mathrm{D}_{\mathrm{v}}=1_{0} \cdot \tan ^{-1}\left\{1 /\left(1+2 \mathrm{~h} / 1_{0}\right)\right\} \quad=1.923 \mathrm{ft}$

$\mathrm{P}_{\mathrm{L}}=0.5 \cdot \mathrm{N}_{\mathrm{SPT}}=0.5 \times 50 \quad=25.000 \mathrm{tsf}=50.000 \mathrm{ksf}$

$\mathrm{H}_{\text {ou }}=0.75 \cdot \mathrm{P}_{\mathrm{L}} \cdot \mathrm{B} \cdot \mathrm{D}_{\mathrm{v}}=0.75 \times 50.000 \times 1.667 \times 1.923=120.188 \mathrm{kips}$

$\varphi \cdot \mathrm{H}_{\text {ou }}=0.750 \times 120.188=90.141$ kips $>\mathrm{H}_{0}=16.025 \mathrm{kips} \quad \therefore$ O.K!

where, $D_{v}=$ zero-shear depth

$\mathrm{P}_{\mathrm{L}} \quad=$ preboring pressuremeter limit pressure within $\mathrm{D}_{\mathrm{v}}$

$\mathrm{H}_{\text {ou }} \quad=$ lateral capacity of pile

2. Lateral Movement at The Ground Surface
$\mathrm{y}_{0}=\left(1+\mathrm{h} / \mathrm{l}_{0}\right) \cdot \mathrm{H}_{0} \cdot \mathrm{l}_{0}{ }^{3} /(2 \mathrm{EI})$
$=0.012 \mathrm{ft}=0.144$ in. $<0.500$ in. $\quad \therefore$ O.K!

3. Lateral Movement at The Pile Head

$\delta=\left\{\left(1+\mathrm{h} / 1_{0}\right)^{3}+0.5\right\} \cdot \mathrm{H}_{0} \cdot 1_{0}{ }^{3} /(3 \mathrm{EI})=0.030 \mathrm{ft}=0.360$ in. $<0.500$ in. $\quad \therefore$ O.K!

4. Maximum Bending Moment

Maximum bending moment can be found by finding the value of $Z_{\max }$ which satisfied $V=0$.

$\mathrm{Z}_{\max }=\mathrm{D}_{\mathrm{v}}=1.923 \mathrm{ft}$

$\mathrm{M}_{\max }=0.5 \cdot \mathrm{H}_{0} 1_{0} \cdot \exp -\left(\mathrm{Z}_{\max } / 1_{0}\right) \cdot \sqrt{ }\left\{\left(1+2 \mathrm{~h} / \mathrm{l}_{0}\right)^{2}+1\right\}$

$=94.050 \mathrm{kips} \cdot \mathrm{ft}$

\section{Check for Creep}

$\mathrm{Pa}=\mathrm{P} / \mathrm{B}$

$=920.000 \times 0.012$

$=11.040 \mathrm{kips} / \mathrm{ft}$

where, $\mathrm{P} \quad=$ the load per unit length of pile

$=6.624 \mathrm{ksf}$

$\mathrm{Pa}=$ average corresponding pressure

F.S $=\mathrm{P}_{\mathrm{L}} / \mathrm{Pa}$

$=50.000 / 6.624$

$=7.548>2 \quad \therefore$ O.K! 


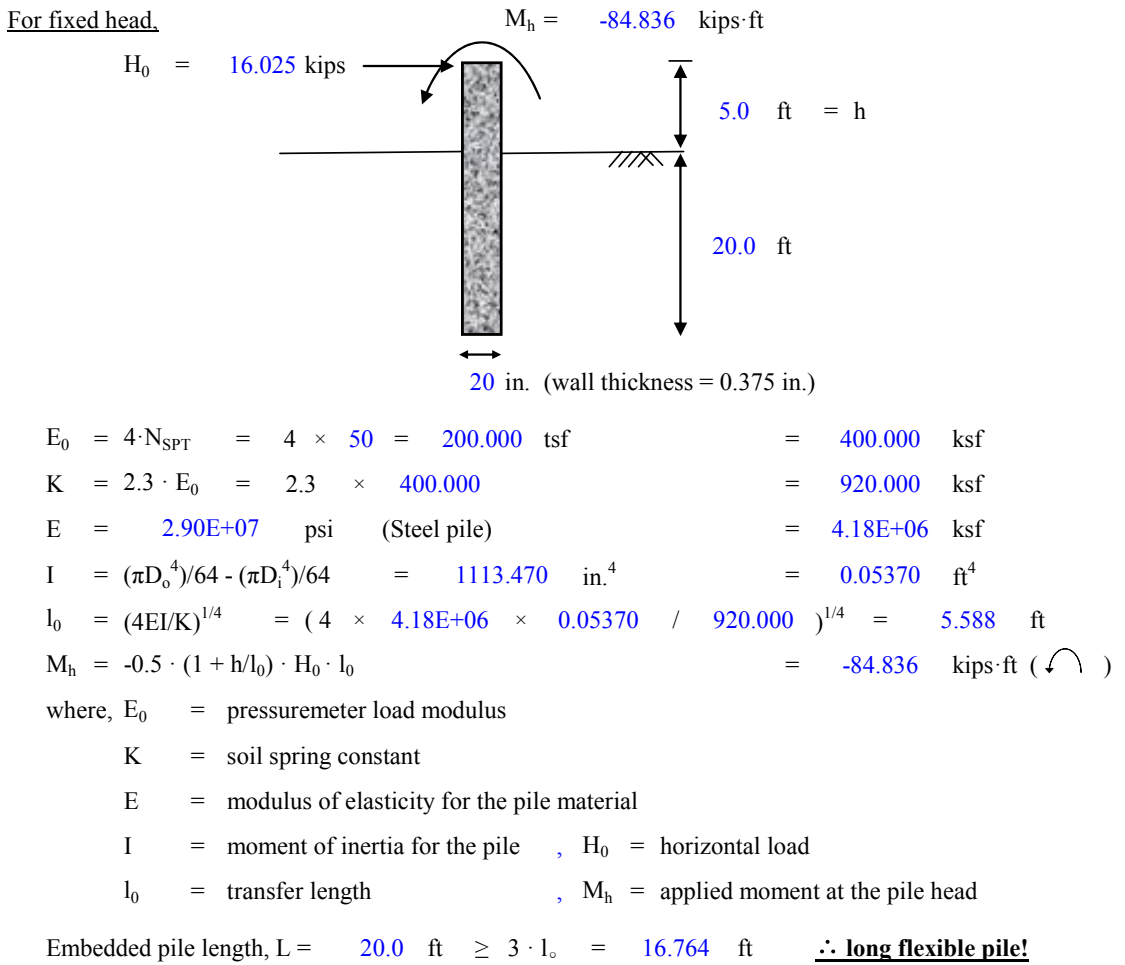

\section{Lateral Pile Capacity}

The zero-shear depth $\mathrm{D}_{\mathrm{v}}$ is obtained by setting $\mathrm{V}$ (shear force $)=0$;

$$
\begin{aligned}
& \mathrm{D}_{\mathrm{v}}=1_{0} \cdot \tan ^{-1}\left(1_{0} / \mathrm{h}\right) \quad=4.699 \mathrm{ft} \\
& \mathrm{P}_{\mathrm{L}}=0.5 \cdot \mathrm{N}_{\mathrm{SPT}}=0.5 \times 50=25.000 \text { tsf }=50.000 \quad \mathrm{ksf} \\
& \mathrm{H}_{\text {ou }}=0.75 \cdot \mathrm{P}_{\mathrm{L}} \cdot \mathrm{B} \cdot \mathrm{D}_{\mathrm{v}}=0.75 \times 50.000 \times 1.667 \times 4.699=293.688 \mathrm{kips} \\
& \varphi \cdot \mathrm{H}_{\text {ou }}=0.750 \times 293.688=220.266 \text { kips }>\mathrm{H}_{0}=16.025 \text { kips } \therefore \text { O.K! }
\end{aligned}
$$

where, $\mathrm{D}_{\mathrm{v}} \quad=$ zero-shear depth

$$
\begin{aligned}
& \mathrm{P}_{\mathrm{L}}=\text { preboring pressuremeter limit pressure within } \mathrm{D}_{\mathrm{v}} \\
& \mathrm{H}_{\mathrm{ou}}=\text { lateral capacity of pile }
\end{aligned}
$$

2. Lateral Movement at The Ground Surface

$$
\mathrm{y}_{0}=\left(1+\mathrm{h} / \mathrm{l}_{0}\right) \cdot \mathrm{H}_{0} \cdot \mathrm{1}_{0}{ }^{3} /(4 \mathrm{EI}) \quad=0.006 \mathrm{ft}=0.072 \text { in. }<0.500 \text { in. } \quad \therefore \text { O.K! }
$$

3. Lateral Movement at The Pile Head

$$
\delta=\left\{\left(1+\mathrm{h} / \mathrm{l}_{0}\right)^{3}+2\right\} \cdot \mathrm{H}_{0} \cdot \mathrm{l}_{0}{ }^{3} /(12 \mathrm{EI})=0.009 \mathrm{ft}=0.108 \text { in. }<0.500 \text { in. } \therefore \text { O.K! }
$$

\section{Maximum Bending Moment}

Maximum bending moment can be found by finding the value of $Z_{\max }$ which satisfied $\mathrm{V}=0$.

$$
\begin{aligned}
\mathrm{Z}_{\max }=\mathrm{D}_{\mathrm{v}} & =4.699 \mathrm{ft} \\
\mathrm{M}_{\max } & =0.5 \cdot \mathrm{H}_{0} \mathrm{1}_{0} \cdot \exp -\left(\mathrm{Z}_{\max } / 1_{0}\right) \cdot \sqrt{ }\left\{1+\left(\mathrm{h} / \mathrm{l}_{0}\right)^{2}\right\} \\
& =25.914 \mathrm{kips} \cdot \mathrm{ft}
\end{aligned}
$$

\section{Check for Creep}

$$
\begin{aligned}
& \mathrm{P}=\mathrm{K} \cdot \mathrm{y}_{0}=920.000 \times 0.006=5.520 \mathrm{kips} / \mathrm{ft} \\
& \mathrm{Pa}=\mathrm{P} / \mathrm{B}=5.520 / 1.667 \\
& \text { where, } \mathrm{P}=\text { the load per unit length of pile } \\
& \qquad \mathrm{Pa}=\text { average corresponding pressure } \\
& \mathrm{F} . \mathrm{S}=\mathrm{P}_{\mathrm{L}} / \mathrm{Pa}=50.000 / 3.312 \mathrm{ksf} \\
&
\end{aligned}
$$




\section{\ Load Combinations (API RP2A - WSD)}

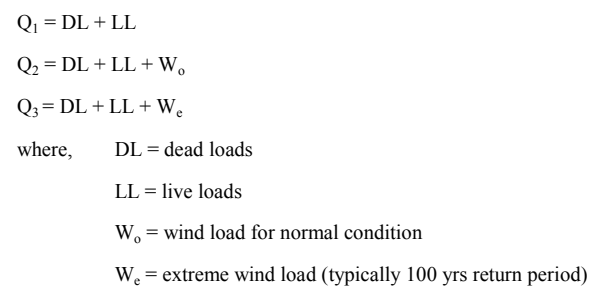

$\mathbf{Q}_{1}=1.00 \times \mathrm{DL}+1.00 \times \mathrm{LL}$

$\Sigma \mathrm{V}=1.00 \times 32.500+1.00 \times 75.833=108.333 \mathrm{kips}$

$\mathbf{Q}_{2}=1.00 \times \mathrm{DL}+1.00 \times \mathrm{LL}+1.00 \times \mathrm{W}_{0}$

$\Sigma \mathrm{V}=1.00 \times 32.500+1.00 \times 75.833=108.333$ kips

$\Sigma \mathrm{H}=1.00 \times(\quad 9.152+8.289+1.099)=18.540$ kips

$\Sigma \mathrm{M}=1.00 \times(\quad 9.152 \times 77.000+8.289 \times 18.000$

$+1.099 \times 2.000)=856.104 \mathrm{kips} \cdot \mathrm{ft}$
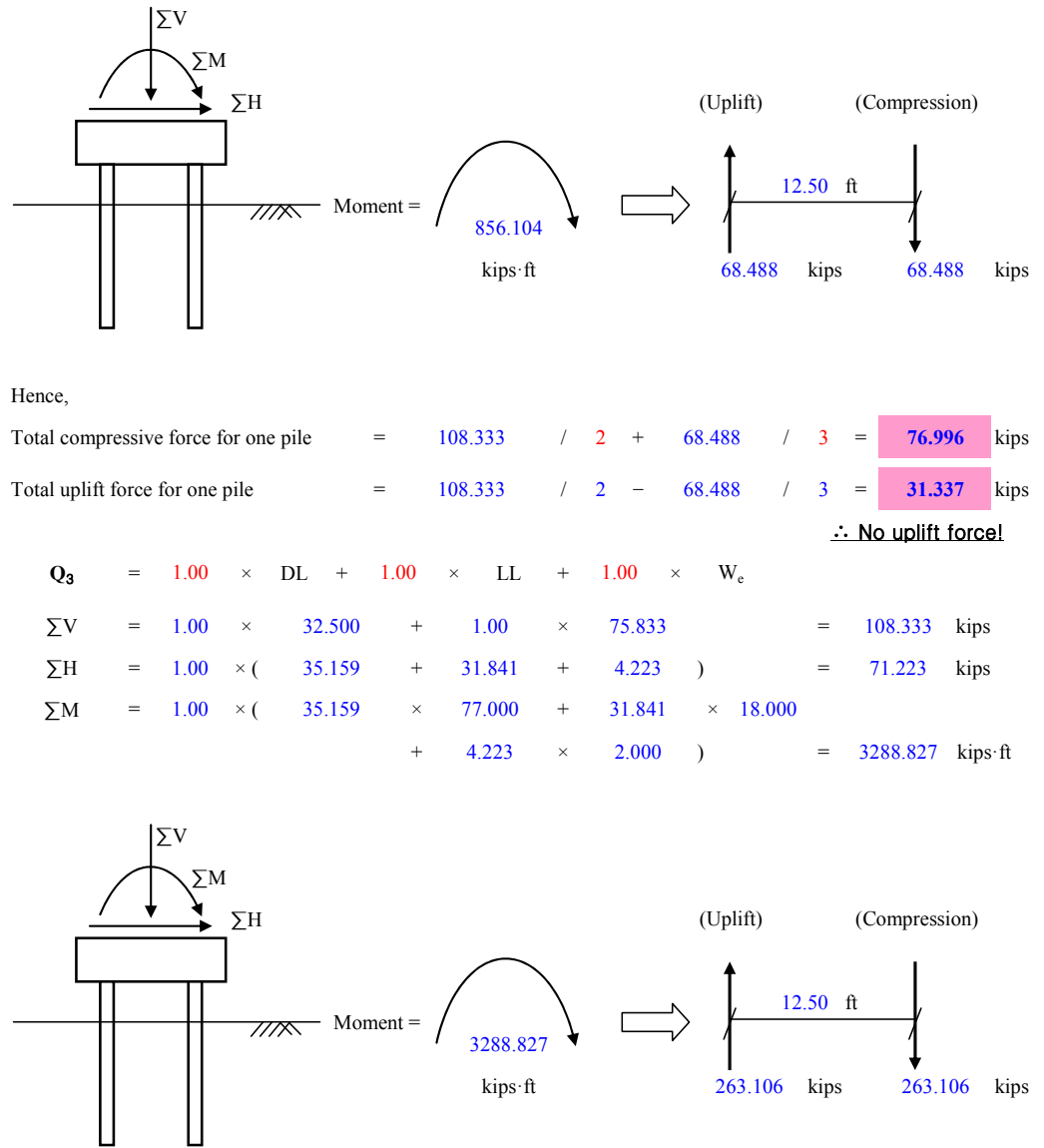

Hence,

$\begin{array}{lllll}\text { Total compressive force for one pile } & =108.333 / 2+263.106 / 3= & \mathbf{1 4 1 . 8 6 9} \text { kips } \\ \text { Total uplift force for one pile } & =108.333 / 2-263.106 / 3=\end{array}$ 


\section{$\square$ Soil Condition}

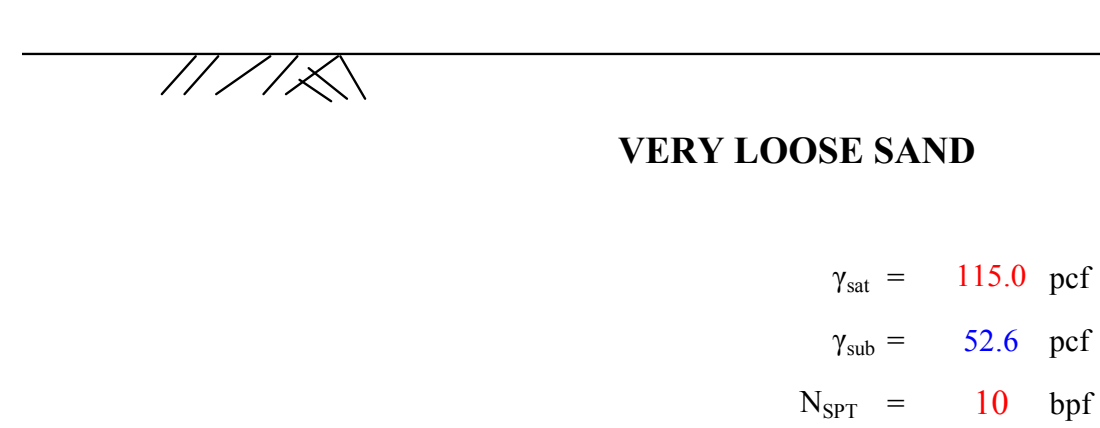

$\square$ Bearing Capacity of the Pile (WSD)

1. Unit end bearing capacity

$\mathrm{q}_{\mathrm{p}} \quad=\mathrm{P}_{\mathrm{o}}{ }^{\prime} \cdot \mathrm{N}_{\mathrm{q}}$

where: $\quad \mathrm{P}_{\mathrm{o}}{ }^{\prime}=$ effective overburden pressure at the point in question

$\mathrm{N}_{\mathrm{q}}=$ dimensionless bearing capacity factor $=8$ (very loose)

2. Unit skin friction capacity

fs $\quad=\mathrm{K} \cdot \mathrm{P}_{\mathrm{o}}{ }^{\prime} \cdot \tan \delta$

where: $\quad \mathrm{K}=$ dimensionless coefficient of lateral earth pressure (ratio of horizontal to vertical normal effective stress) $=1.00$ (full displacement piles, plugged or closed end)

$\delta=$ friction angle between the soil and pile wall $=15^{\circ}$ (very loose)

3. Ultimate bearing capacity

$\mathrm{Qu}=\mathrm{Qp}+\mathrm{Qs} \quad=\mathrm{q}_{\mathrm{p}} \mathrm{Ap}+\mathrm{fs} A \mathrm{~s}$

where: $\quad A p=$ gross end area of pile $=\pi \mathrm{D}^{2} / 4$ (where, $\mathrm{D}$ : diameter of pile)

As $=$ side surface area of pile $=\pi D L$ (where, $L$ : length of pile)

4. Allowable bearing capacity

$\mathrm{Qa}=\mathrm{Qu} /$ Factor of Safety 
1. Operating Environmental Conditions

Factor of Safety $=\quad 2.0$

Diameter, $D=12$ inches

\begin{tabular}{|c|c|c|c|c|c|c|c|c|}
\hline Depth [ft] & $\mathrm{P}_{\mathrm{o}}{ }^{\prime}[\mathrm{psf}]$ & $\mathrm{q}_{\mathrm{p}}[\mathrm{ksf}]$ & Qp [kips] & fs [ksf] & $\Sigma \mathrm{Qs}[\mathrm{kips}]$ & Qu [kips] & Qa [kips] & Qa (uplift) \\
\hline 0 & 0 & 0 & 0 & 0 & 0 & 0 & 0 & 0 \\
\hline 10 & 526.000 & 4.208 & 3.305 & 0.070 & 2.199 & 5.504 & 2.752 & 1.100 \\
\hline 20 & 1052.000 & 8.416 & 6.610 & 0.141 & 8.859 & 15.469 & 7.735 & 4.430 \\
\hline 30 & 1578.000 & 12.624 & 9.915 & 0.211 & 19.886 & 29.801 & 14.901 & 9.943 \\
\hline 40 & 2104.000 & 16.832 & 13.220 & 0.282 & 35.437 & 48.657 & 24.329 & 17.719 \\
\hline 50 & 2630.000 & 21.040 & 16.525 & 0.352 & 55.292 & 71.817 & 35.909 & 27.646 \\
\hline 60 & 3156.000 & 25.248 & 19.830 & 0.423 & 79.734 & 99.564 & 49.782 & 39.867 \\
\hline 70 & 3682.000 & 29.456 & 23.135 & 0.493 & 108.416 & 131.551 & 65.776 & 54.208 \\
\hline 80 & 4208.000 & 33.664 & 26.440 & 0.557 & 139.876 & 166.316 & 83.158 & 69.938 \\
\hline
\end{tabular}

Diameter, $D=16$ inches

\begin{tabular}{|l|c|c|c|c|c|c|c|c|}
\hline Depth [ft] & $\mathrm{P}_{\mathrm{o}}{ }^{\prime}[\mathrm{psf}]$ & $\mathrm{q}_{\mathrm{p}}[\mathrm{ksf}]$ & Qp [kips] & fs [ksf] & IQs [kips] & Qu [kips] & Qa [kips] & Qa (uplift) \\
\hline
\end{tabular}

\begin{tabular}{|c|c|c|c|c|c|c|c|c|}
\hline 0 & 0 & 0 & 0 & 0 & 0 & 0 & 0 & 0 \\
\hline 10 & 526.000 & 4.208 & 5.875 & 0.070 & 2.932 & 8.807 & 4.404 & 1.466 \\
\hline 20 & 1052.000 & 8.416 & 11.751 & 0.141 & 11.812 & 23.563 & 11.782 & 5.906 \\
\hline 30 & 1578.000 & 12.624 & 17.626 & 0.211 & 26.515 & 44.141 & 22.071 & 13.258 \\
\hline 40 & 2104.000 & 16.832 & 23.502 & 0.282 & 47.250 & 70.752 & 35.376 & 23.625 \\
\hline 50 & 2630.000 & 21.040 & 29.377 & 0.352 & 73.723 & 103.100 & 51.550 & 36.862 \\
\hline 60 & 3156.000 & 25.248 & 35.253 & 0.423 & 106.311 & 141.564 & 70.782 & 53.156 \\
\hline 70 & 3682.000 & 29.456 & 41.128 & 0.493 & 144.555 & 185.683 & 92.842 & 72.278 \\
\hline 80 & 4208.000 & 33.664 & 47.004 & 0.557 & 186.502 & 233.506 & 116.753 & 93.251 \\
\hline
\end{tabular}

Diameter, $D=20$ inches

\begin{tabular}{|c|c|c|c|c|c|c|c|c|}
\hline Depth [ft] & $\mathrm{P}_{\mathrm{o}}{ }^{\prime}[\mathrm{psf}]$ & $\mathrm{q}_{\mathrm{p}}[\mathrm{ksf}]$ & Qp [kips] & fs [ksf] & IQs [kips] & Qu [kips] & Qa [kips] & Qa (uplift) \\
\hline 0 & 0 & 0 & 0 & 0 & 0 & 0 & 0 & 0 \\
\hline 10 & 526.000 & 4.208 & 9.180 & 0.070 & 3.665 & 12.845 & 6.423 & 1.833 \\
\hline 20 & 1052.000 & 8.416 & 18.361 & 0.141 & 14.765 & 33.126 & 16.563 & 7.383 \\
\hline 30 & 1578.000 & 12.624 & 27.541 & 0.211 & 33.144 & 60.685 & 30.343 & 16.572 \\
\hline 40 & 2104.000 & 16.832 & 36.722 & 0.282 & 59.062 & 95.784 & 47.892 & 29.531 \\
\hline 50 & 2630.000 & 21.040 & 45.902 & 0.352 & 92.153 & 138.055 & 69.028 & 46.077 \\
\hline 60 & 3156.000 & 25.248 & 55.083 & 0.423 & 132.889 & 187.972 & 93.986 & 66.445 \\
\hline 70 & 3682.000 & 29.456 & 64.263 & 0.493 & 180.694 & 244.957 & 122.479 & 90.347 \\
\hline 80 & 4208.000 & 33.664 & 73.443 & 0.557 & 233.127 & 306.570 & 153.285 & 116.564 \\
\hline
\end{tabular}

Diameter, $D=24$ inches

\begin{tabular}{|c|c|c|c|c|c|c|c|c|}
\hline Depth [ft] & $\mathrm{P}_{\mathrm{o}}{ }^{\prime}[\mathrm{psf}]$ & $\mathrm{q}_{\mathrm{p}}[\mathrm{ksf}]$ & Qp [kips] & $\mathrm{fs}[\mathrm{ksf}]$ & $\Sigma \mathrm{Qs}[\mathrm{kips}]$ & Qu [kips] & Qa [kips] & Qa (uplift) \\
\hline 0 & 0 & 0 & 0 & 0 & 0 & 0 & 0 & 0 \\
\hline 10 & 526.000 & 4.208 & 13.220 & 0.070 & 4.398 & 17.618 & 8.809 & 2.199 \\
\hline 20 & 1052.000 & 8.416 & 26.440 & 0.141 & 17.719 & 44.159 & 22.080 & 8.860 \\
\hline 30 & 1578.000 & 12.624 & 39.659 & 0.211 & 39.773 & 79.432 & 39.716 & 19.887 \\
\hline 40 & 2104.000 & 16.832 & 52.879 & 0.282 & 70.874 & 123.753 & 61.877 & 35.437 \\
\hline 50 & 2630.000 & 21.040 & 66.099 & 0.352 & 110.584 & 176.683 & 88.342 & 55.292 \\
\hline 60 & 3156.000 & 25.248 & 79.319 & 0.423 & 159.467 & 238.786 & 119.393 & 79.734 \\
\hline 70 & 3682.000 & 29.456 & 92.539 & 0.493 & 216.833 & 309.372 & 154.686 & 108.417 \\
\hline 80 & 4208.000 & 33.664 & 105.759 & 0.557 & 279.753 & 385.512 & 192.756 & 139.877 \\
\hline
\end{tabular}

Qa [kips] for operating condition

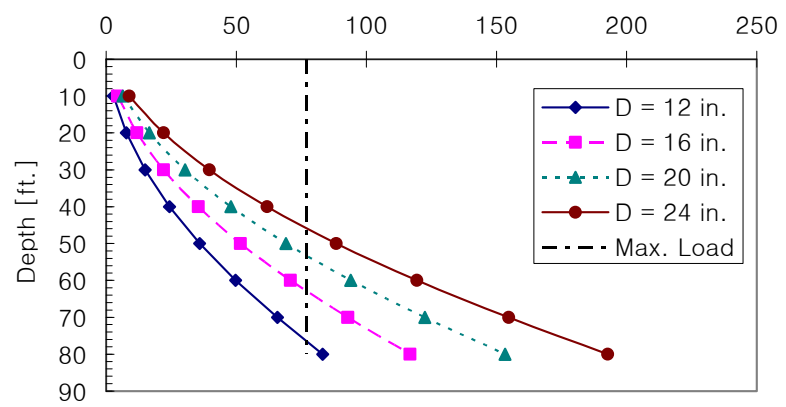


2. Extreme Environmental Conditions

Factor of Safety $=$

1.5

2.0 (uplift)

Diameter, $D=12$ inches

\begin{tabular}{|c|c|c|c|c|c|c|c|c|}
\hline Depth [ft] & $\mathrm{P}_{\mathrm{o}}{ }^{\prime}[\mathrm{psf}]$ & $\mathrm{q}_{\mathrm{p}}[\mathrm{ksf}]$ & Qp [kips] & $\mathrm{fs}[\mathrm{ksf}]$ & $\Sigma \mathrm{Qs}[\mathrm{kips}]$ & $\mathrm{Qu}[\mathrm{kips}]$ & Qa [kips] & Qa (uplift) \\
\hline 0 & 0 & 0 & 0 & 0 & 0 & 0 & 0 & 0 \\
\hline 10 & 526.000 & 4.208 & 3.305 & 0.070 & 2.199 & 5.504 & 3.669 & 1.100 \\
\hline 20 & 1052.000 & 8.416 & 6.610 & 0.141 & 8.859 & 15.469 & 10.313 & 4.430 \\
\hline 30 & 1578.000 & 12.624 & 9.915 & 0.211 & 19.886 & 29.801 & 19.867 & 9.943 \\
\hline 40 & 2104.000 & 16.832 & 13.220 & 0.282 & 35.437 & 48.657 & 32.438 & 17.719 \\
\hline 50 & 2630.000 & 21.040 & 16.525 & 0.352 & 55.292 & 71.817 & 47.878 & 27.646 \\
\hline 60 & 3156.000 & 25.248 & 19.830 & 0.423 & 79.734 & 99.564 & 66.376 & 39.867 \\
\hline 70 & 3682.000 & 29.456 & 23.135 & 0.493 & 108.416 & 131.551 & 87.701 & 54.208 \\
\hline 80 & 4208.000 & 33.664 & 26.440 & 0.557 & 139.876 & 166.316 & 110.877 & 69.938 \\
\hline
\end{tabular}

Diameter, $D=16$ inches

\begin{tabular}{|c|c|c|c|c|c|c|c|c|}
\hline Depth [ft] & $\mathrm{P}_{\mathrm{o}}{ }^{\prime}[\mathrm{psf}]$ & $\mathrm{q}_{\mathrm{p}}[\mathrm{ksf}]$ & Qp [kips] & $\mathrm{fs}[\mathrm{ksf}]$ & $\Sigma \mathrm{Qs}[\mathrm{kips}]$ & Qu [kips] & Qa [kips] & Qa (uplift) \\
\hline 0 & 0 & 0 & 0 & 0 & 0 & 0 & 0 & 0 \\
\hline 10 & 526.000 & 4.208 & 5.875 & 0.070 & 2.932 & 8.807 & 5.871 & 1.466 \\
\hline 20 & 1052.000 & 8.416 & 11.751 & 0.141 & 11.812 & 23.563 & 15.709 & 5.906 \\
\hline 30 & 1578.000 & 12.624 & 17.626 & 0.211 & 26.515 & 44.141 & 29.427 & 13.258 \\
\hline 40 & 2104.000 & 16.832 & 23.502 & 0.282 & 47.250 & 70.752 & 47.168 & 23.625 \\
\hline 50 & 2630.000 & 21.040 & 29.377 & 0.352 & 73.723 & 103.100 & 68.733 & 36.862 \\
\hline 60 & 3156.000 & 25.248 & 35.253 & 0.423 & 106.311 & 141.564 & 94.376 & 53.156 \\
\hline 70 & 3682.000 & 29.456 & 41.128 & 0.493 & 144.555 & 185.683 & 123.789 & 72.278 \\
\hline 80 & 4208.000 & 33.664 & 47.004 & 0.557 & 186.502 & 233.506 & 155.671 & 93.251 \\
\hline
\end{tabular}

Diameter, $D=20$ inches

\begin{tabular}{|l|c|c|c|c|c|c|c|c|}
\hline Depth [ft] & $\mathrm{P}_{\mathrm{o}}^{\prime}$ [psf] & $\mathrm{q}_{\mathrm{p}}$ [ksf] & Qp [kips] & fs [ksf] & $\Sigma \mathrm{Qs}$ [kips] & Qu [kips] & Qa [kips] & Qa (uplift) \\
\hline
\end{tabular}

\begin{tabular}{|c|c|c|c|c|c|c|c|c|}
\hline 0 & 0 & 0 & 0 & 0 & 0 & 0 & 0 & 0 \\
\hline 10 & 526.000 & 4.208 & 9.180 & 0.070 & 3.665 & 12.845 & 8.563 & 1.833 \\
\hline 20 & 1052.000 & 8.416 & 18.361 & 0.141 & 14.765 & 33.126 & 22.084 & 7.383 \\
\hline 30 & 1578.000 & 12.624 & 27.541 & 0.211 & 33.144 & 60.685 & 40.457 & 16.572 \\
\hline 40 & 2104.000 & 16.832 & 36.722 & 0.282 & 59.062 & 95.784 & 63.856 & 29.531 \\
\hline 50 & 2630.000 & 21.040 & 45.902 & 0.352 & 92.153 & 138.055 & 92.037 & 46.077 \\
\hline 60 & 3156.000 & 25.248 & 55.083 & 0.423 & 132.889 & 187.972 & 125.315 & 66.445 \\
\hline 70 & 3682.000 & 29.456 & 64.263 & 0.493 & 180.694 & 244.957 & 163.305 & 90.347 \\
\hline 80 & 4208.000 & 33.664 & 73.443 & 0.557 & 233.127 & 306.570 & 204.380 & 116.564 \\
\hline
\end{tabular}

Diameter, $D=24$ inches

\begin{tabular}{|c|c|c|c|c|c|c|c|c|}
\hline Depth [ft] & $\mathrm{P}_{\mathrm{o}}{ }^{\prime}[\mathrm{psf}]$ & $\mathrm{q}_{\mathrm{p}}[\mathrm{ksf}]$ & $\mathrm{Qp}[\mathrm{kips}]$ & $\mathrm{fs}[\mathrm{ksf}]$ & $\Sigma \mathrm{Qs}[\mathrm{kips}]$ & Qu [kips] & Qa [kips] & Qa (uplift) \\
\hline 0 & 0 & 0 & 0 & 0 & 0 & 0 & 0 & 0 \\
\hline 10 & 526.000 & 4.208 & 13.220 & 0.070 & 4.398 & 17.618 & 11.745 & 2.199 \\
\hline 20 & 1052.000 & 8.416 & 26.440 & 0.141 & 17.719 & 44.159 & 29.439 & 8.860 \\
\hline 30 & 1578.000 & 12.624 & 39.659 & 0.211 & 39.773 & 79.432 & 52.955 & 19.887 \\
\hline 40 & 2104.000 & 16.832 & 52.879 & 0.282 & 70.874 & 123.753 & 82.502 & 35.437 \\
\hline 50 & 2630.000 & 21.040 & 66.099 & 0.352 & 110.584 & 176.683 & 117.789 & 55.292 \\
\hline 60 & 3156.000 & 25.248 & 79.319 & 0.423 & 159.467 & 238.786 & 159.191 & 79.734 \\
\hline 70 & 3682.000 & 29.456 & 92.539 & 0.493 & 216.833 & 309.372 & 206.248 & 108.417 \\
\hline 80 & 4208.000 & 33.664 & 105.759 & 0.557 & 279.753 & 385.512 & 257.008 & 139.877 \\
\hline
\end{tabular}

Qa [kips] for extreme condition

Qa(uplift) [kips] for extreme condition
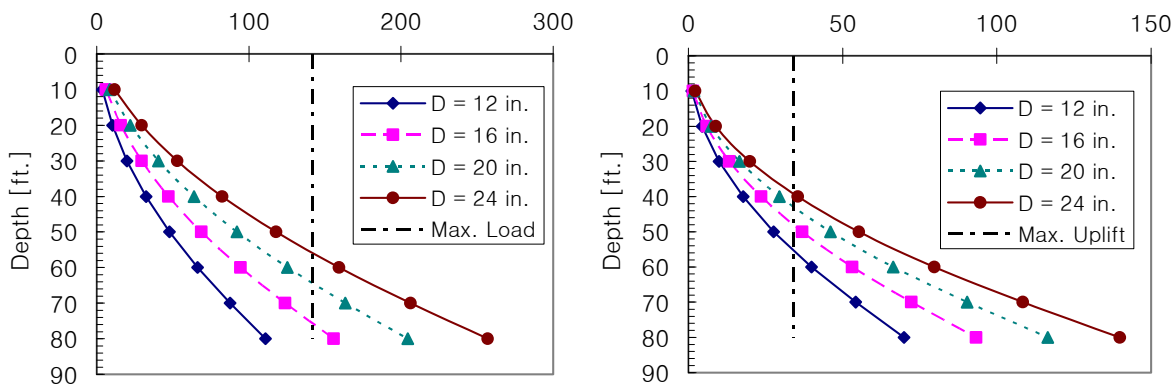
$\square$ Horizontal Capacity of the Pile (SALLOP)

For free head,

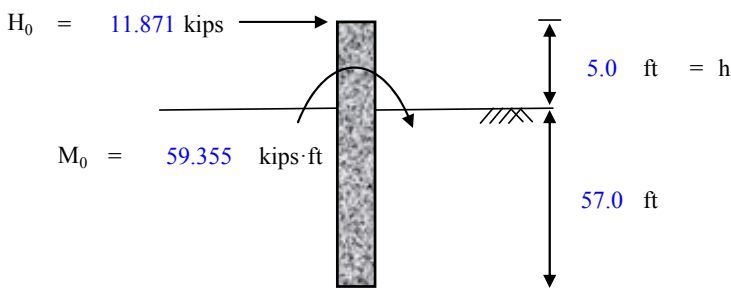

24 in. (wall thickness $=0.375$ in.)

$$
\begin{aligned}
& \mathrm{E}_{0}=4 \cdot \mathrm{N}_{\mathrm{SPT}}=4 \times 10=40.000 \text { tsf }=80.000 \mathrm{ksf} \\
& \mathrm{K}=2.3 \cdot \mathrm{E}_{0}=2.3 \times 80.000=184.000 \mathrm{ksf} \\
& \mathrm{E}=2.90 \mathrm{E}+07 \quad \mathrm{psi} \quad \text { (Steel pile) } \quad=4.18 \mathrm{E}+06 \mathrm{ksf} \\
& \mathrm{I}=\left(\pi \mathrm{D}_{\mathrm{o}}{ }^{4}\right) / 64-\left(\pi \mathrm{D}_{\mathrm{i}}^{4}\right) / 64=1942.299 \mathrm{in}^{4}=0.09367 \mathrm{ft}^{4} \\
& 1_{0}=(4 \mathrm{EI} / \mathrm{K})^{1 / 4}=(4 \times 4.18 \mathrm{E}+06 \times 0.09367 / 184.000)^{1 / 4}=9.603 \mathrm{ft}
\end{aligned}
$$

\section{Lateral Pile Capacity}

The zero-shear depth $\mathrm{D}_{\mathrm{v}}$ is obtained by setting $\mathrm{V}$ ( shear force) $=0$;

$$
\begin{aligned}
& \mathrm{D}_{\mathrm{v}}=1_{0} \cdot \tan ^{-1}\left\{1 /\left(1+2 \mathrm{~h} / \mathrm{l}_{0}\right)\right\} \quad=4.374 \mathrm{ft} \\
& \mathrm{P}_{\mathrm{L}}=0.5 \cdot \mathrm{N}_{\mathrm{SPT}}=0.5 \times 10=5.000 \text { tsf }=10.000 \quad \mathrm{ksf} \\
& \mathrm{H}_{\text {ou }}=0.75 \cdot \mathrm{P}_{\mathrm{L}} \cdot \mathrm{B} \cdot \mathrm{D}_{\mathrm{v}}=0.75 \times 10.000 \times 2.000 \times 4.374=65.610 \mathrm{kips} \\
& \mathrm{H}_{\text {allow }}=65.610 / 3.000=21.870 \text { kips }>\mathrm{H}_{0}=11.871 \mathrm{kips} \quad \therefore \text { O.K! }
\end{aligned}
$$

where, $\mathrm{D}_{\mathrm{v}} \quad=$ zero-shear depth

$$
\begin{aligned}
& \mathrm{P}_{\mathrm{L}}=\text { preboring pressuremeter limit pressure within } \mathrm{D}_{\mathrm{v}} \\
& \mathrm{H}_{\mathrm{ou}}=\text { lateral capacity of pile }
\end{aligned}
$$

2. Lateral Movement at The Ground Surface

$$
\mathrm{y}_{0}=\left(1+\mathrm{h} / 1_{0}\right) \cdot \mathrm{H}_{0} \cdot 1_{0}{ }^{3} /(2 \mathrm{EI}) \quad=0.020 \mathrm{ft}=0.240 \text { in. }<0.500 \text { in. } \quad \therefore \text { O.K! }
$$

\section{Lateral Movement at The Pile Head}

$$
\delta=\left\{\left(1+\mathrm{h} / \mathrm{l}_{0}\right)^{3}+0.5\right\} \cdot \mathrm{H}_{0} \cdot 1_{0}{ }^{3} /(3 \mathrm{EI})=0.036 \mathrm{ft}=0.432 \text { in. }<0.500 \text { in. } \quad \therefore \text { O.K! }
$$

\section{Maximum Bending Moment}

Maximum bending moment can be found by finding the value of $\mathrm{Z}_{\max }$ which satisfied $\mathrm{V}=0$.

$$
\mathrm{Z}_{\max }=\mathrm{D}_{\mathrm{v}}=4.374 \mathrm{ft}
$$$$
\mathrm{M}_{\max }=0.5 \cdot \mathrm{H}_{0} \mathrm{l}_{0} \cdot \exp -\left(\mathrm{Z}_{\max } / \mathrm{l}_{0}\right) \cdot \sqrt{ }\left\{\left(1+2 \mathrm{~h} / \mathrm{l}_{0}\right)^{2}+1\right\}
$$

$$
=82.162 \mathrm{kips} \cdot \mathrm{ft}
$$

\section{Check for Creep}

$$
\begin{aligned}
& \mathrm{P}=\mathrm{K} \cdot \mathrm{y}_{0}=184.000 \times 0.020=3.680 \mathrm{kips} / \mathrm{ft} \\
& \mathrm{Pa}=\mathrm{P} / \mathrm{B}=3.680 / 2.000=1.840 \mathrm{ksf} \\
& \text { where, } \mathrm{P}=\text { the load per unit length of pile } \\
& \mathrm{Pa} \quad=\text { average corresponding pressure } \\
& \mathrm{F} . \mathrm{S}=\mathrm{P}_{\mathrm{L}} / \mathrm{Pa}=10.000 / 1.840=5.435>2 \quad \therefore \quad \text { O.K! }
\end{aligned}
$$




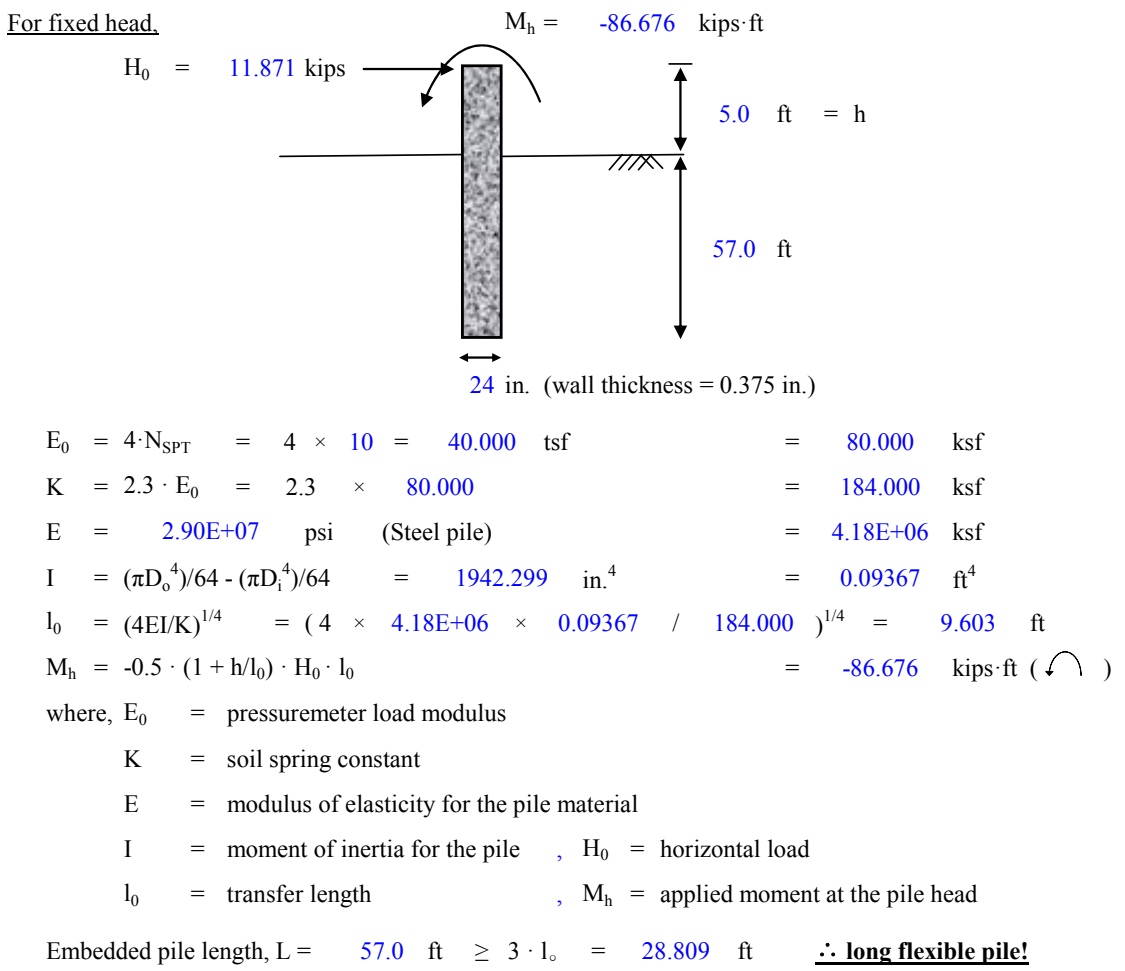

\section{Lateral Pile Capacity}

The zero-shear depth $\mathrm{D}_{\mathrm{v}}$ is obtained by setting $\mathrm{V}$ (shear force) $=0$;

$$
\begin{aligned}
& \mathrm{D}_{\mathrm{v}}=1_{0} \cdot \tan ^{-1}\left(1_{0} / \mathrm{h}\right) \quad=10.474 \mathrm{ft} \\
& \mathrm{P}_{\mathrm{L}}=0.5 \cdot \mathrm{N}_{\mathrm{SPT}}=0.5 \times 10=5.000 \text { tsf }=10.000 \quad \mathrm{ksf} \\
& \mathrm{H}_{\text {ou }}=0.75 \cdot \mathrm{P}_{\mathrm{L}} \cdot \mathrm{B} \cdot \mathrm{D}_{\mathrm{v}}=0.75 \times 10.000 \times 2.000 \times 10.474=157.110 \mathrm{kips} \\
& \mathrm{H}_{\text {allow }}=157.110 / 3.000=52.370 \text { kips }>\mathrm{H}_{0}=11.871 \text { kips } \quad \therefore \text { O.K! }
\end{aligned}
$$

where, $D_{v}=$ zero-shear depth

$$
\begin{aligned}
& \mathrm{P}_{\mathrm{L}}=\text { preboring pressuremeter limit pressure within } \mathrm{D}_{\mathrm{v}} \\
& \mathrm{H}_{\text {ou }}=\text { lateral capacity of pile }
\end{aligned}
$$

\section{Lateral Movement at The Ground Surface}

$$
\mathrm{y}_{0}=\left(1+\mathrm{h} / \mathrm{l}_{0}\right) \cdot \mathrm{H}_{0} \cdot \mathrm{l}_{0}{ }^{3} /(4 \mathrm{EI}) \quad=0.010 \mathrm{ft}=0.120 \text { in. }<0.500 \text { in. } \quad \therefore \text { O.K! }
$$

3. Lateral Movement at The Pile Head

$$
\delta=\left\{\left(1+\mathrm{h} / \mathrm{l}_{0}\right)^{3}+2\right\} \cdot \mathrm{H}_{0} \cdot \mathrm{l}_{0}{ }^{3} /(12 \mathrm{EI})=0.012 \mathrm{ft}=0.144 \text { in. }<0.500 \text { in. } \therefore \text { O.K! }
$$

\section{Maximum Bending Moment}

Maximum bending moment can be found by finding the value of $Z_{\max }$ which satisfied $V=0$.

$$
\begin{aligned}
\mathrm{Z}_{\max }=\mathrm{D}_{\mathrm{v}} & =10.474 \mathrm{ft} \\
\mathrm{M}_{\max } & =0.5 \cdot \mathrm{H}_{0} \mathrm{l}_{0} \cdot \exp -\left(\mathrm{Z}_{\max } / 1_{0}\right) \cdot \sqrt{ }\left\{1+\left(\mathrm{h} / \mathrm{l}_{0}\right)^{2}\right\} \\
& =21.591 \quad \mathrm{kips} \cdot \mathrm{ft}
\end{aligned}
$$

\section{Check for Creep}

$$
\begin{aligned}
& \mathrm{P}=\mathrm{K} \cdot \mathrm{y}_{0}=184.000 \times 0.010=1.840 \mathrm{kips} / \mathrm{ft} \\
& \mathrm{Pa}=\mathrm{P} / \mathrm{B}=1.840 / 2.000=0.920 \mathrm{ksf} \\
& \text { where, } \mathrm{P}=\text { the load per unit length of pile } \\
& \qquad \mathrm{Pa}=\text { average corresponding pressure } \\
& \mathrm{F} . \mathrm{S}=\mathrm{P}_{\mathrm{L}} / \mathrm{Pa}=10.000 / 0.920=10.870>2 \quad \therefore \text { O.K! }
\end{aligned}
$$


Soil Condition

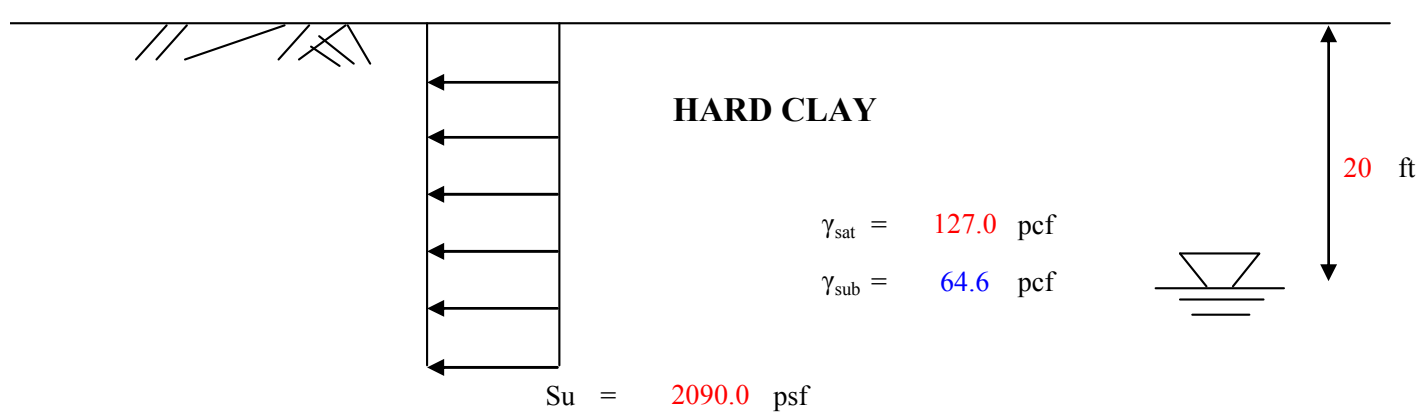

Bearing Capacity of the Pile (ADSC-LRFD)

1. Unit end bearing capacity

$\mathrm{q}_{\mathrm{p}}=9 \cdot \mathrm{Su}$ (If Su $\geq 2000 \mathrm{psf}$, and depth of base $\geq 3 \mathrm{~B}, \mathrm{~B}=$ diameter of pile)

$=\quad \mathrm{N}_{\mathrm{c}}^{*} \cdot \mathrm{Su}($ If Su $<2000 \mathrm{psf}$, and depth of base $\geq 3 \mathrm{~B}, \mathrm{~B}=$ diameter of pile)

$=(2 / 3)[1+(1 / 6)(\mathrm{D} / \mathrm{B})] \mathrm{N}^{*} \mathrm{c} \cdot \mathrm{Su}($ If depth of base $(\mathrm{D})<3 \mathrm{~B})$

2. Unit skin friction capacity
$\mathrm{fs}=\alpha \cdot \mathrm{Su}$
$\alpha=0.55$ (for $\mathrm{Su} / \mathrm{Pa} \leq 1.5$ and varying linearly between 0.55 and 0.45 for $1.5<\mathrm{Su} / \mathrm{Pa}<2.5$ )
$\alpha=0 \quad$ (for top five feet, and bottom one diameter)
where: $\quad \alpha=$ a dimensionless adhesion factor
$\mathrm{Pa}=$ the atmospheric pressure $=2116 \mathrm{psf}$

3. Ultimate bearing capacity

$$
\begin{array}{ll}
\mathrm{Qu}= & \mathrm{Qp}+\mathrm{Qs} \quad=\mathrm{q}_{\mathrm{p}} \mathrm{Ap}+\mathrm{fs} \mathrm{As} \\
\text { where: } & \mathrm{Ap}=\text { gross end area of pile }=\pi \mathrm{D}^{2} / 4 \text { (where, } \mathrm{D}: \text { diameter of pile) } \\
& \mathrm{As}=\text { side surface area of pile }=\pi \mathrm{DL} \text { (where, L: length of pile) }
\end{array}
$$


1. Operating Environmental Conditions

Resistance Factors $(\varphi)$ for end bearing $=\quad 0.55$, skin friction $=\quad 0.65$, uplift $=\quad 0.55$

Diameter, $D=12$ inches

\begin{tabular}{|c|c|c|c|c|c|c|c|c|c|}
\hline Depth [ft] & $\mathrm{N}^{*}{ }_{\mathrm{c}}$ & $\mathrm{q}_{\mathrm{p}}[\mathrm{ksf}]$ & Qp [kips] & $\alpha$ & $\mathrm{fs}[\mathrm{ksf}]$ & $\Sigma \mathrm{Qs}[\mathrm{kips}]$ & Qu [kips] & $\varphi$ Qu [kips] & $\varphi$ Qu(uplift) \\
\hline 0 & 0 & 0 & 0 & 0 & 0 & 0 & 0 & 0 & 0 \\
\hline 5 & 9.00 & 18.810 & 14.773 & 0.00 & 0.000 & 0.000 & 14.773 & 8.125 & 0.000 \\
\hline 10 & 9.00 & 18.810 & 14.773 & 0.55 & 1.150 & 14.451 & 29.224 & 17.518 & 7.948 \\
\hline 15 & 9.00 & 18.810 & 14.773 & 0.55 & 1.150 & 32.515 & 47.288 & 29.260 & 17.883 \\
\hline 20 & 9.00 & 18.810 & 14.773 & 0.55 & 1.150 & 50.580 & 65.353 & 41.002 & 27.819 \\
\hline 25 & 9.00 & 18.810 & 14.773 & 0.55 & 1.150 & 68.644 & 83.417 & 52.744 & 37.754 \\
\hline 30 & 9.00 & 18.810 & 14.773 & 0.55 & 1.150 & 86.708 & 101.481 & 64.485 & 47.689 \\
\hline 35 & 9.00 & 18.810 & 14.773 & 0.55 & 1.150 & 104.772 & 119.545 & 76.227 & 57.625 \\
\hline 40 & 9.00 & 18.810 & 14.773 & 0.55 & 1.150 & 122.836 & 137.609 & 87.969 & 67.560 \\
\hline
\end{tabular}

Diameter, $D=16$ inches

\begin{tabular}{|c|c|c|c|c|c|c|c|c|c|}
\hline Depth [ft] & $\mathrm{N}^{*}{ }_{\mathrm{c}}$ & $\mathrm{q}_{\mathrm{p}}[\mathrm{ksf}]$ & Qp [kips] & $\alpha$ & $\mathrm{fs}[\mathrm{ksf}]$ & $\Sigma \mathrm{Qs}[\mathrm{kips}]$ & Qu [kips] & $\varphi \mathrm{Qu}[\mathrm{kips}]$ & $\varphi \mathrm{Qu}($ uplift $)$ \\
\hline 0 & 0 & 0 & 0 & 0 & 0 & 0 & 0 & 0 & 0 \\
\hline 5 & 9.00 & 18.810 & 26.264 & 0.00 & 0.000 & 0.000 & 26.264 & 14.445 & 0.000 \\
\hline 10 & 9.00 & 18.810 & 26.264 & 0.55 & 1.150 & 17.663 & 43.927 & 25.926 & 9.715 \\
\hline 15 & 9.00 & 18.810 & 26.264 & 0.55 & 1.150 & 41.748 & 68.012 & 41.581 & 22.961 \\
\hline 20 & 9.00 & 18.810 & 26.264 & 0.55 & 1.150 & 65.834 & 92.098 & 57.237 & 36.209 \\
\hline 25 & 9.00 & 18.810 & 26.264 & 0.55 & 1.150 & 89.919 & 116.183 & 72.893 & 49.455 \\
\hline 30 & 9.00 & 18.810 & 26.264 & 0.55 & 1.150 & 114.005 & 140.269 & 88.548 & 62.703 \\
\hline 35 & 9.00 & 18.810 & 26.264 & 0.55 & 1.150 & 138.090 & 164.354 & 104.204 & 75.950 \\
\hline 40 & 9.00 & 18.810 & 26.264 & 0.55 & 1.150 & 162.176 & 188.440 & 119.860 & 89.197 \\
\hline
\end{tabular}

Diameter, $D=20$ inches

\begin{tabular}{|c|c|c|c|c|c|c|c|c|c|}
\hline Depth [ft] & $\mathrm{N}^{*}{ }_{\mathrm{c}}$ & $\mathrm{q}_{\mathrm{p}}[\mathrm{ksf}]$ & $\mathrm{Qp}[\mathrm{kips}]$ & $\alpha$ & $\mathrm{fs}[\mathrm{ksf}]$ & $\Sigma \mathrm{Qs}[\mathrm{kips}]$ & $\mathrm{Qu}[\mathrm{kips}]$ & $\varphi \mathrm{Qu}[\mathrm{kips}]$ & $\varphi \mathrm{Qu}($ uplift) \\
\hline 0 & 0 & 0 & 0 & 0 & 0 & 0 & 0 & 0 & 0 \\
\hline 5 & 9.00 & 18.810 & 41.037 & 0.00 & 0.000 & 0.000 & 41.037 & 22.570 & 0.000 \\
\hline 10 & 9.00 & 18.810 & 41.037 & 0.55 & 1.150 & 20.071 & 61.108 & 35.617 & 11.039 \\
\hline 15 & 9.00 & 18.810 & 41.037 & 0.55 & 1.150 & 50.178 & 91.215 & 55.186 & 27.598 \\
\hline 20 & 9.00 & 18.810 & 41.037 & 0.55 & 1.150 & 80.285 & 121.322 & 74.756 & 44.157 \\
\hline 25 & 9.00 & 18.810 & 41.037 & 0.55 & 1.150 & 110.392 & 151.429 & 94.325 & 60.716 \\
\hline 30 & 9.00 & 18.810 & 41.037 & 0.55 & 1.150 & 140.499 & 181.536 & 113.895 & 77.274 \\
\hline 35 & 9.00 & 18.810 & 41.037 & 0.55 & 1.150 & 170.606 & 211.643 & 133.464 & 93.833 \\
\hline 40 & 9.00 & 18.810 & 41.037 & 0.55 & 1.150 & 200.713 & 241.750 & 153.034 & 110.392 \\
\hline
\end{tabular}

Diameter, $D=24$ inches

\begin{tabular}{|c|c|c|c|c|c|c|c|c|c|}
\hline Depth [ft] & $\mathrm{N}^{*}{ }_{\mathrm{c}}$ & $\mathrm{q}_{\mathrm{p}}[\mathrm{ksf}]$ & $\mathrm{Qp}[\mathrm{kips}]$ & $\alpha$ & $\mathrm{fs}[\mathrm{ksf}]$ & $\Sigma \mathrm{Qs}[\mathrm{kips}]$ & $\mathrm{Qu}[\mathrm{kips}]$ & $\varphi \mathrm{Qu}[\mathrm{kips}]$ & $\varphi \mathrm{Qu}$ (uplift) \\
\hline 0 & 0 & 0 & 0 & 0 & 0 & 0 & 0 & 0 & 0 \\
\hline 5 & 9.00 & 17.765 & 55.810 & 0.00 & 0.000 & 0.000 & 55.810 & 30.696 & 0.000 \\
\hline 10 & 9.00 & 18.810 & 59.093 & 0.55 & 1.150 & 21.677 & 80.770 & 46.591 & 11.922 \\
\hline 15 & 9.00 & 18.810 & 59.093 & 0.55 & 1.150 & 57.805 & 116.898 & 70.074 & 31.793 \\
\hline 20 & 9.00 & 18.810 & 59.093 & 0.55 & 1.150 & 93.934 & 153.027 & 93.558 & 51.664 \\
\hline 25 & 9.00 & 18.810 & 59.093 & 0.55 & 1.150 & 130.062 & 189.155 & 117.041 & 71.534 \\
\hline 30 & 9.00 & 18.810 & 59.093 & 0.55 & 1.150 & 166.190 & 225.283 & 140.525 & 91.405 \\
\hline 35 & 9.00 & 18.810 & 59.093 & 0.55 & 1.150 & 202.319 & 261.412 & 164.009 & 111.275 \\
\hline 40 & 9.00 & 18.810 & 59.093 & 0.55 & 1.150 & 238.447 & 297.540 & 187.492 & 131.146 \\
\hline
\end{tabular}

$\phi Q u[k i p s]$ for operating condition

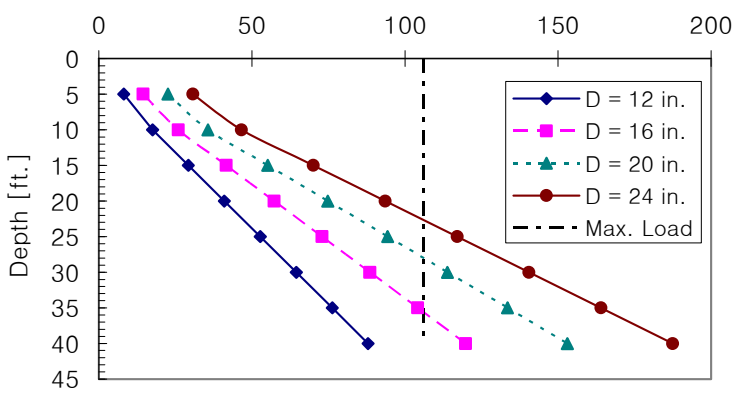


2. Extreme Environmental Conditions

Resistance Factors, $\varphi=$

1.0

1.0 (uplift)

Diameter, $D=12$ inches

\begin{tabular}{|c|c|c|c|c|c|c|c|c|c|}
\hline Depth [ft] & $\mathrm{N}_{\mathrm{c}}$ & $\mathrm{q}_{\mathrm{p}}[\mathrm{ksf}]$ & Qp [kips] & $\alpha$ & $\mathrm{fs}[\mathrm{ksf}]$ & $\Sigma \mathrm{Qs}[\mathrm{kips}]$ & Qu [kips] & $\varphi \mathrm{Qu}[\mathrm{kips}]$ & $\varphi \mathrm{Qu}($ uplift) \\
\hline 0 & 0 & 0 & 0 & 0 & 0 & 0 & 0 & 0 & 0 \\
\hline 5 & 9.00 & 18.810 & 14.773 & 0.00 & 0.000 & 0.000 & 14.773 & 14.773 & 0.000 \\
\hline 10 & 9.00 & 18.810 & 14.773 & 0.55 & 1.150 & 14.451 & 29.224 & 29.224 & 14.451 \\
\hline 15 & 9.00 & 18.810 & 14.773 & 0.55 & 1.150 & 32.515 & 47.288 & 47.288 & 32.515 \\
\hline 20 & 9.00 & 18.810 & 14.773 & 0.55 & 1.150 & 50.580 & 65.353 & 65.353 & 50.580 \\
\hline 25 & 9.00 & 18.810 & 14.773 & 0.55 & 1.150 & 68.644 & 83.417 & 83.417 & 68.644 \\
\hline 30 & 9.00 & 18.810 & 14.773 & 0.55 & 1.150 & 86.708 & 101.481 & 101.481 & 86.708 \\
\hline 35 & 9.00 & 18.810 & 14.773 & 0.55 & 1.150 & 104.772 & 119.545 & 119.545 & 104.772 \\
\hline 40 & 9.00 & 18.810 & 14.773 & 0.55 & 1.150 & 122.836 & 137.609 & 137.609 & 122.836 \\
\hline
\end{tabular}

Diameter, $D=16$ inches

\begin{tabular}{|c|c|c|c|c|c|c|c|c|c|}
\hline Depth [ft] & $\mathrm{N}_{\mathrm{c}}$ & $\mathrm{q}_{\mathrm{p}}[\mathrm{ksf}]$ & Qp [kips] & $\alpha$ & $\mathrm{fs}[\mathrm{ksf}]$ & $\Sigma \mathrm{Qs}[\mathrm{kips}]$ & Qu [kips] & $\varphi \mathrm{Qu}[\mathrm{kips}]$ & $\varphi \mathrm{Qu}(\mathrm{uplift})$ \\
\hline 0 & 0 & 0 & 0 & 0 & 0 & 0 & 0 & 0 & 0 \\
\hline 5 & 9.00 & 18.810 & 26.264 & 0.00 & 0.000 & 0.000 & 26.264 & 26.264 & 0.000 \\
\hline 10 & 9.00 & 18.810 & 26.264 & 0.55 & 1.150 & 17.663 & 43.927 & 43.927 & 17.663 \\
\hline 15 & 9.00 & 18.810 & 26.264 & 0.55 & 1.150 & 41.748 & 68.012 & 68.012 & 41.748 \\
\hline 20 & 9.00 & 18.810 & 26.264 & 0.55 & 1.150 & 65.834 & 92.098 & 92.098 & 65.834 \\
\hline 25 & 9.00 & 18.810 & 26.264 & 0.55 & 1.150 & 89.919 & 116.183 & 116.183 & 89.919 \\
\hline 30 & 9.00 & 18.810 & 26.264 & 0.55 & 1.150 & 114.005 & 140.269 & 140.269 & 114.005 \\
\hline 35 & 9.00 & 18.810 & 26.264 & 0.55 & 1.150 & 138.090 & 164.354 & 164.354 & 138.090 \\
\hline 40 & 9.00 & 18.810 & 26.264 & 0.55 & 1.150 & 162.176 & 188.440 & 188.440 & 162.176 \\
\hline
\end{tabular}

Diameter, $D=20$ inches

\begin{tabular}{|c|c|c|c|c|c|c|c|c|c|}
\hline Depth [ft] & $\mathrm{N}_{\mathrm{c}}$ & $\mathrm{q}_{\mathrm{p}}[\mathrm{ksf}]$ & Qp [kips] & $\alpha$ & $\mathrm{fs}[\mathrm{ksf}]$ & $\Sigma \mathrm{Qs}[\mathrm{kips}]$ & Qu [kips] & $\varphi \mathrm{Qu}[\mathrm{kips}]$ & $\varphi$ Qu(uplift) \\
\hline 0 & 0 & 0 & 0 & 0 & 0 & 0 & 0 & 0 & 0 \\
\hline 5 & 9.00 & 18.810 & 41.037 & 0.00 & 0.000 & 0.000 & 41.037 & 41.037 & 0.000 \\
\hline 10 & 9.00 & 18.810 & 41.037 & 0.55 & 1.150 & 20.071 & 61.108 & 61.108 & 20.071 \\
\hline 15 & 9.00 & 18.810 & 41.037 & 0.55 & 1.150 & 50.178 & 91.215 & 91.215 & 50.178 \\
\hline 20 & 9.00 & 18.810 & 41.037 & 0.55 & 1.150 & 80.285 & 121.322 & 121.322 & 80.285 \\
\hline 25 & 9.00 & 18.810 & 41.037 & 0.55 & 1.150 & 110.392 & 151.429 & 151.429 & 110.392 \\
\hline 30 & 9.00 & 18.810 & 41.037 & 0.55 & 1.150 & 140.499 & 181.536 & 181.536 & 140.499 \\
\hline 35 & 9.00 & 18.810 & 41.037 & 0.55 & 1.150 & 170.606 & 211.643 & 211.643 & 170.606 \\
\hline 40 & 9.00 & 18.810 & 41.037 & 0.55 & 1.150 & 200.713 & 241.750 & 241.750 & 200.713 \\
\hline
\end{tabular}

Diameter, $D=24$ inches

\begin{tabular}{|c|c|c|c|c|c|c|c|c|c|}
\hline Depth [ft] & $\mathrm{N}_{\mathrm{c}}$ & $\mathrm{q}_{\mathrm{p}}[\mathrm{ksf}]$ & Qp [kips] & $\alpha$ & $\mathrm{fs}[\mathrm{ksf}]$ & $\Sigma \mathrm{Qs}[\mathrm{kips}]$ & $\mathrm{Qu}[\mathrm{kips}]$ & $\varphi \mathrm{Qu}[\mathrm{kips}]$ & $\varphi \mathrm{Qu}($ uplift) \\
\hline 0 & 0 & 0 & 0 & 0 & 0 & 0 & 0 & 0 & 0 \\
\hline 5 & 9.00 & 17.765 & 55.810 & 0.00 & 0.000 & 0.000 & 55.810 & 55.810 & 0.000 \\
\hline 10 & 9.00 & 18.810 & 59.093 & 0.55 & 1.150 & 21.677 & 80.770 & 80.770 & 21.677 \\
\hline 15 & 9.00 & 18.810 & 59.093 & 0.55 & 1.150 & 57.805 & 116.898 & 116.898 & 57.805 \\
\hline 20 & 9.00 & 18.810 & 59.093 & 0.55 & 1.150 & 93.934 & 153.027 & 153.027 & 93.934 \\
\hline 25 & 9.00 & 18.810 & 59.093 & 0.55 & 1.150 & 130.062 & 189.155 & 189.155 & 130.062 \\
\hline 30 & 9.00 & 18.810 & 59.093 & 0.55 & 1.150 & 166.190 & 225.283 & 225.283 & 166.190 \\
\hline 35 & 9.00 & 18.810 & 59.093 & 0.55 & 1.150 & 202.319 & 261.412 & 261.412 & 202.319 \\
\hline 40 & 9.00 & 18.810 & 59.093 & 0.55 & 1.150 & 238.447 & 297.540 & 297.540 & 238.447 \\
\hline
\end{tabular}

$\phi Q u$ [kips] for extreme condition

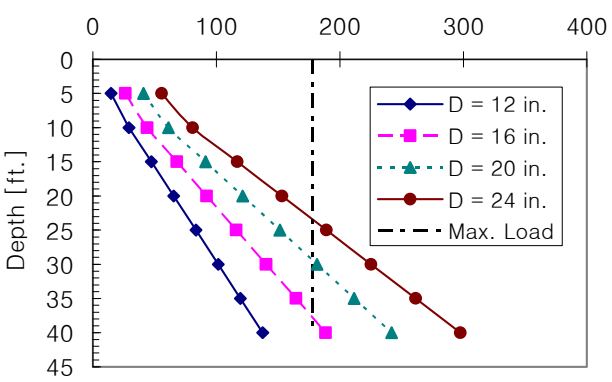

$\phi Q u(u p l i f t)[k i p s]$ for extreme condition

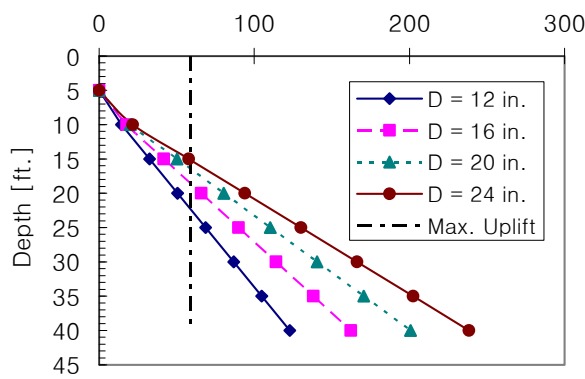


Soil Condition

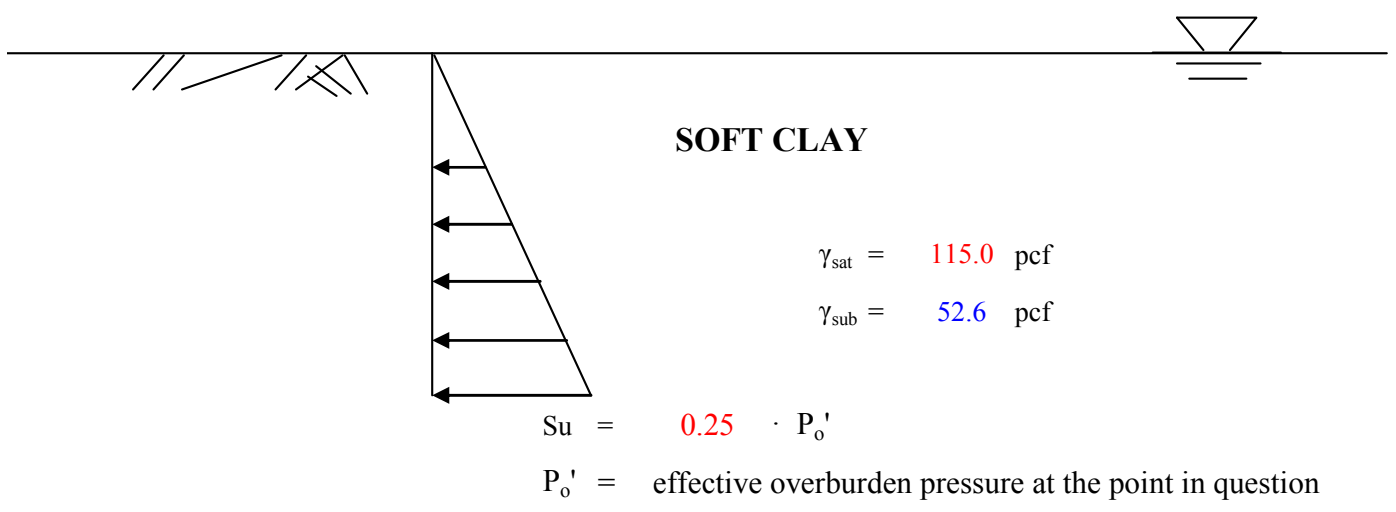

\section{$\square$ Bearing Capacity of the Pile (ADSC-WSD)}

1. Unit end bearing capacity

$\mathrm{q}_{\mathrm{p}}=9 \cdot \mathrm{Su}$ (If $\mathrm{Su} \geq 2000 \mathrm{psf}$, and depth of base $\geq 3 \mathrm{~B}, \mathrm{~B}=$ diameter of pile)

$=\mathrm{N}_{\mathrm{c}}^{*} \cdot \mathrm{Su}$ (If Su $<2000$ psf, and depth of base $\geq 3 \mathrm{~B}, \mathrm{~B}=$ diameter of pile)

$=(2 / 3)[1+(1 / 6)(\mathrm{D} / \mathrm{B})] \mathrm{N}^{*} \mathrm{c} \cdot \mathrm{Su}($ If depth of base $(\mathrm{D})<3 \mathrm{~B})$

2. Unit skin friction capacity
fs $=\alpha \cdot \mathrm{Su}$
$\alpha=0.55$ (for $\mathrm{Su} / \mathrm{Pa} \leq 1.5$ and varying linearly between 0.55 and 0.45 for $1.5<\mathrm{Su} / \mathrm{Pa}<2.5$ )
$\alpha=0 \quad$ (for top five feet, and bottom one diameter)
where: $\alpha=$ a dimensionless adhesion factor
$\mathrm{Pa}=$ the atmospheric pressure $=2116 \mathrm{psf}$

\section{Ultimate bearing capacity}

$$
\begin{aligned}
\mathrm{Qu}=\mathrm{Qp}+\mathrm{Qs}=\mathrm{q}_{\mathrm{p}} \mathrm{Ap}+\mathrm{fsAs} \\
\text { where: } \mathrm{Ap}=\text { gross end area of pile }=\pi \mathrm{D}^{2} / 4 \text { (where, } \mathrm{D} \text { : diameter of pile) } \\
\mathrm{As}=\text { side surface area of pile }=\pi \mathrm{DL} \text { (where, } \mathrm{L} \text { : length of pile) }
\end{aligned}
$$

\section{Allowable bearing capacity}

$\mathrm{Qa}=\mathrm{Qu} /$ Factor of Safety 
1. Operating Environmental Conditions

Factor of Safety $=\quad 3.0$

Diameter, $D=12$ inches

\begin{tabular}{|c|c|c|c|c|c|c|c|c|c|c|}
\hline Depth [ft] & $\mathrm{Su}[\mathrm{ksf}]$ & $\mathrm{N}_{\mathrm{c}}$ & $\mathrm{q}_{\mathrm{p}}[\mathrm{ksf}]$ & $\mathrm{Qp}[\mathrm{kips}]$ & $\alpha$ & $\mathrm{fs}[\mathrm{ksf}]$ & $\Sigma \mathrm{Qs}[\mathrm{kips}]$ & Qu [kips] & Qa [kips] & Qa (uplift) \\
\hline 0 & 0 & 0 & 0 & 0 & 0 & 0 & 0 & 0 & 0 & 0 \\
\hline 20 & 0.263 & 6.50 & 1.710 & 1.343 & 0.55 & 0.073 & 3.211 & 4.554 & 1.518 & 1.070 \\
\hline 40 & 0.526 & 6.58 & 3.460 & 2.717 & 0.55 & 0.145 & 15.488 & 18.205 & 6.068 & 5.163 \\
\hline 60 & 0.789 & 7.37 & 5.813 & 4.566 & 0.55 & 0.217 & 36.813 & 41.379 & 13.793 & 12.271 \\
\hline 80 & 1.052 & 8.05 & 8.471 & 6.653 & 0.55 & 0.289 & 67.186 & 73.839 & 24.613 & 22.395 \\
\hline 100 & 1.315 & 8.32 & 10.934 & 8.588 & 0.55 & 0.362 & 106.902 & 115.490 & 38.497 & 35.634 \\
\hline 120 & 1.578 & 8.58 & 13.536 & 10.631 & 0.55 & 0.434 & 155.433 & 166.064 & 55.355 & 51.811 \\
\hline 140 & 1.841 & 8.84 & 16.276 & 12.783 & 0.55 & 0.507 & 213.434 & 226.217 & 75.406 & 71.145 \\
\hline 160 & 2.104 & 9.00 & 18.936 & 14.872 & 0.55 & 0.579 & 280.123 & 294.995 & 98.332 & 93.374 \\
\hline
\end{tabular}

Diameter, $D=16$ inches

\begin{tabular}{|c|c|c|c|c|c|c|c|c|c|c|}
\hline Depth [f] & Su $[\mathrm{ksf}]$ & $\mathrm{N}_{\mathrm{c}}^{*}$ & $\mathrm{q}_{\mathrm{p}}[\mathrm{ksf}]$ & Qp [kips] & $\alpha$ & $\mathrm{fs}[\mathrm{ksf}]$ & $\Sigma \mathrm{Qs}[\mathrm{kips}]$ & Qu [kips] & Qa [kips] & Qa (uplift) \\
\hline 0 & 0 & 0 & 0 & 0 & 0 & 0 & 0 & 0 & 0 & 0 \\
\hline 20 & 0.263 & 6.50 & 1.710 & 2.388 & 0.55 & 0.073 & 4.179 & 6.567 & 2.189 & 1.393 \\
\hline 40 & 0.526 & 6.58 & 3.460 & 4.831 & 0.55 & 0.145 & 20.448 & 25.279 & 8.426 & 6.816 \\
\hline 60 & 0.789 & 7.37 & 5.813 & 8.116 & 0.55 & 0.217 & 48.781 & 56.897 & 18.966 & 16.260 \\
\hline 80 & 1.052 & 8.05 & 8.471 & 11.828 & 0.55 & 0.289 & 89.178 & 101.006 & 33.669 & 29.726 \\
\hline 100 & 1.315 & 8.32 & 10.934 & 15.267 & 0.55 & 0.362 & 142.031 & 157.298 & 52.433 & 47.344 \\
\hline 120 & 1.578 & 8.58 & 13.536 & 18.900 & 0.55 & 0.434 & 206.639 & 225.539 & 75.180 & 68.880 \\
\hline 140 & 1.841 & 8.84 & 16.276 & 22.726 & 0.55 & 0.507 & 283.870 & 306.596 & 102.199 & 94.623 \\
\hline 160 & 2.104 & 9.00 & 18.936 & 26.440 & 0.55 & 0.579 & 372.689 & 399.129 & 133.043 & 124.230 \\
\hline
\end{tabular}

Diameter, $D=20$ inches

\begin{tabular}{|c|c|c|c|c|c|c|c|c|c|c|}
\hline Depth [ft] & Su [ksf] & $\mathrm{N}^{*}{ }_{\mathrm{c}}$ & $\mathrm{q}_{\mathrm{p}}[\mathrm{ksf}]$ & Qp [kips] & $\alpha$ & $\mathrm{fs}[\mathrm{ksf}]$ & $\Sigma \mathrm{Qs}[\mathrm{kips}]$ & Qu [kips] & Qa [kips] & Qa (uplift) \\
\hline 0 & 0 & 0 & 0 & 0 & 0 & 0 & 0 & 0 & 0 & 0 \\
\hline 20 & 0.263 & 6.50 & 1.710 & 3.731 & 0.55 & 0.073 & 5.096 & 8.827 & 2.942 & 1.699 \\
\hline 40 & 0.526 & 6.58 & 3.460 & 7.549 & 0.55 & 0.145 & 25.307 & 32.856 & 10.952 & 8.436 \\
\hline 60 & 0.789 & 7.37 & 5.813 & 12.682 & 0.55 & 0.217 & 60.598 & 73.280 & 24.427 & 20.199 \\
\hline 80 & 1.052 & 8.05 & 8.471 & 18.481 & 0.55 & 0.289 & 110.968 & 129.449 & 43.150 & 36.989 \\
\hline 100 & 1.315 & 8.32 & 10.934 & 23.854 & 0.55 & 0.362 & 176.907 & 200.761 & 66.920 & 58.969 \\
\hline 120 & 1.578 & 8.58 & 13.536 & 29.531 & 0.55 & 0.434 & 257.541 & 287.072 & 95.691 & 85.847 \\
\hline 140 & 1.841 & 8.84 & 16.276 & 35.509 & 0.55 & 0.507 & 353.953 & 389.462 & 129.821 & 117.984 \\
\hline 160 & 2.104 & 9.00 & 18.936 & 41.312 & 0.55 & 0.579 & 464.851 & 506.163 & 168.721 & 154.950 \\
\hline
\end{tabular}

Diameter, $D=24$ inches

\begin{tabular}{|c|c|c|c|c|c|c|c|c|c|c|}
\hline Depth [ft] & Su $[\mathrm{ksf}]$ & $\mathrm{N}_{\mathrm{c}}$ & $\mathrm{q}_{\mathrm{p}}[\mathrm{ksf}]$ & Qp $[\mathrm{kips}]$ & $\alpha$ & $\mathrm{fs}[\mathrm{ksf}]$ & $\Sigma \mathrm{Qs}[\mathrm{kips}]$ & Qu [kips $]$ & Qa [kips] & Qa (uplift) \\
\hline 0 & 0 & 0 & 0 & 0 & 0 & 0 & 0 & 0 & 0 & 0 \\
\hline 20 & 0.263 & 6.50 & 1.710 & 5.372 & 0.55 & 0.073 & 5.963 & 11.335 & 3.778 & 1.988 \\
\hline 40 & 0.526 & 6.58 & 3.460 & 10.870 & 0.55 & 0.145 & 30.065 & 40.935 & 13.645 & 10.022 \\
\hline 60 & 0.789 & 7.37 & 5.813 & 18.262 & 0.55 & 0.217 & 72.263 & 90.525 & 30.175 & 24.088 \\
\hline 80 & 1.052 & 8.05 & 8.471 & 26.612 & 0.55 & 0.289 & 132.556 & 159.168 & 53.056 & 44.185 \\
\hline 100 & 1.315 & 8.32 & 10.934 & 34.350 & 0.55 & 0.362 & 211.530 & 245.880 & 81.960 & 70.510 \\
\hline 120 & 1.578 & 8.58 & 13.536 & 42.525 & 0.55 & 0.434 & 308.140 & 350.665 & 116.888 & 102.713 \\
\hline 140 & 1.841 & 8.84 & 16.276 & 51.133 & 0.55 & 0.507 & 423.681 & 474.814 & 158.271 & 141.227 \\
\hline 160 & 2.104 & 9.00 & 18.936 & 59.489 & 0.55 & 0.579 & 556.609 & 616.098 & 205.366 & 185.536 \\
\hline
\end{tabular}

Qa [kips] for operating condition

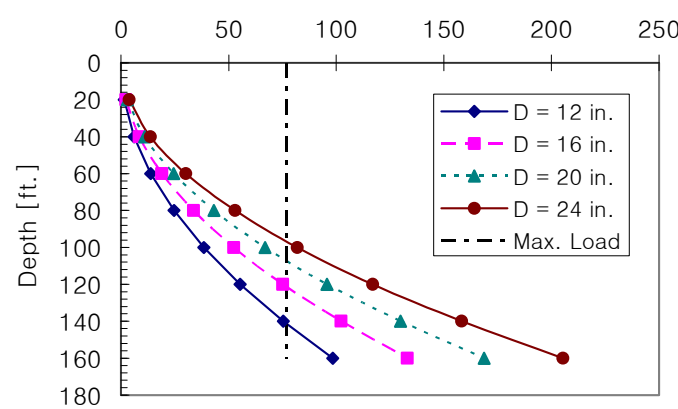


2. Extreme Environmental Conditions

Factor of Safety $=\quad 2.0$

3.0 (uplift)

Diameter, $D=12$ inches

\begin{tabular}{|c|c|c|c|c|c|c|c|c|c|c|}
\hline Depth [ft] & Su [ksf] & $\mathrm{N}_{\mathrm{c}}$ & $\mathrm{q}_{\mathrm{p}}[\mathrm{ksf}]$ & Qp [kips] & $\alpha$ & $\mathrm{fs}[\mathrm{ksf}]$ & $\Sigma$ Qs [kips] & Qu [kips] & Qa [kips] & Qa (uplift) \\
\hline 0 & 0 & 0 & 0 & 0 & 0 & 0 & 0 & 0 & 0 & 0 \\
\hline 20 & 0.263 & 6.50 & 1.710 & 1.343 & 0.55 & 0.073 & 3.211 & 4.554 & 2.277 & 1.070 \\
\hline 40 & 0.526 & 6.58 & 3.460 & 2.717 & 0.55 & 0.145 & 15.488 & 18.205 & 9.103 & 5.163 \\
\hline 60 & 0.789 & 7.37 & 5.813 & 4.566 & 0.55 & 0.217 & 36.813 & 41.379 & 20.690 & 12.271 \\
\hline 80 & 1.052 & 8.05 & 8.471 & 6.653 & 0.55 & 0.289 & 67.186 & 73.839 & 36.920 & 22.395 \\
\hline 100 & 1.315 & 8.32 & 10.934 & 8.588 & 0.55 & 0.362 & 106.902 & 115.490 & 57.745 & 35.634 \\
\hline 120 & 1.578 & 8.58 & 13.536 & 10.631 & 0.55 & 0.434 & 155.433 & 166.064 & 83.032 & 51.811 \\
\hline 140 & 1.841 & 8.84 & 16.276 & 12.783 & 0.55 & 0.507 & 213.434 & 226.217 & 113.109 & 71.145 \\
\hline 160 & 2.104 & 9.00 & 18.936 & 14.872 & 0.55 & 0.579 & 280.123 & 294.995 & 147.498 & 93.374 \\
\hline
\end{tabular}

Diameter, $D=16$ inches

\begin{tabular}{|c|c|c|c|c|c|c|c|c|c|c|}
\hline Depth [ft] & Su [ksf] & $\mathrm{N}_{\mathrm{c}}^{*}$ & $\mathrm{q}_{\mathrm{p}}[\mathrm{ksf}]$ & Qp [kips] & $\alpha$ & $\mathrm{fs}[\mathrm{ksf}]$ & $\Sigma \mathrm{Qs}[\mathrm{kips}]$ & Qu [kips] & Qa [kips] & Qa (uplift) \\
\hline 0 & 0 & 0 & 0 & 0 & 0 & 0 & 0 & 0 & 0 & 0 \\
\hline 20 & 0.263 & 6.50 & 1.710 & 2.388 & 0.55 & 0.073 & 4.179 & 6.567 & 3.284 & 1.393 \\
\hline 40 & 0.526 & 6.58 & 3.460 & 4.831 & 0.55 & 0.145 & 20.448 & 25.279 & 12.640 & 6.816 \\
\hline 60 & 0.789 & 7.37 & 5.813 & 8.116 & 0.55 & 0.217 & 48.781 & 56.897 & 28.449 & 16.260 \\
\hline 80 & 1.052 & 8.05 & 8.471 & 11.828 & 0.55 & 0.289 & 89.178 & 101.006 & 50.503 & 29.726 \\
\hline 100 & 1.315 & 8.32 & 10.934 & 15.267 & 0.55 & 0.362 & 142.031 & 157.298 & 78.649 & 47.344 \\
\hline 120 & 1.578 & 8.58 & 13.536 & 18.900 & 0.55 & 0.434 & 206.639 & 225.539 & 112.770 & 68.880 \\
\hline 140 & 1.841 & 8.84 & 16.276 & 22.726 & 0.55 & 0.507 & 283.870 & 306.596 & 153.298 & 94.623 \\
\hline 160 & 2.104 & 9.00 & 18.936 & 26.440 & 0.55 & 0.579 & 372.689 & 399.129 & 199.565 & 124.230 \\
\hline
\end{tabular}

Diameter, $D=20$ inches

\begin{tabular}{|c|c|c|c|c|c|c|c|c|c|c|}
\hline Depth [ft] & Su [ksf] & $\mathrm{N}_{\mathrm{c}}$ & $\mathrm{q}_{\mathrm{p}}[\mathrm{ksf}]$ & $\mathrm{Qp}[\mathrm{kips}]$ & $\alpha$ & $\mathrm{fs}[\mathrm{ksf}]$ & $\Sigma \mathrm{Qs}[\mathrm{kips}]$ & $\mathrm{Qu}[\mathrm{kips}]$ & $\mathrm{Qa}[\mathrm{kips}]$ & Qa (uplift) \\
\hline 0 & 0 & 0 & 0 & 0 & 0 & 0 & 0 & 0 & 0 & 0 \\
\hline 20 & 0.263 & 6.50 & 1.710 & 3.731 & 0.55 & 0.073 & 5.096 & 8.827 & 4.414 & 1.699 \\
\hline 40 & 0.526 & 6.58 & 3.460 & 7.549 & 0.55 & 0.145 & 25.307 & 32.856 & 16.428 & 8.436 \\
\hline 60 & 0.789 & 7.37 & 5.813 & 12.682 & 0.55 & 0.217 & 60.598 & 73.280 & 36.640 & 20.199 \\
\hline 80 & 1.052 & 8.05 & 8.471 & 18.481 & 0.55 & 0.289 & 110.968 & 129.449 & 64.725 & 36.989 \\
\hline 100 & 1.315 & 8.32 & 10.934 & 23.854 & 0.55 & 0.362 & 176.907 & 200.761 & 100.381 & 58.969 \\
\hline 120 & 1.578 & 8.58 & 13.536 & 29.531 & 0.55 & 0.434 & 257.541 & 287.072 & 143.536 & 85.847 \\
\hline 140 & 1.841 & 8.84 & 16.276 & 35.509 & 0.55 & 0.507 & 353.953 & 389.462 & 194.731 & 117.984 \\
\hline 160 & 2.104 & 9.00 & 18.936 & 41.312 & 0.55 & 0.579 & 464.851 & 506.163 & 253.082 & 154.950 \\
\hline
\end{tabular}

Diameter, $D=24$ inches

\begin{tabular}{|c|c|c|c|c|c|c|c|c|c|c|}
\hline Depth [ft] & Su [ksf] & $\mathrm{N}^{*}{ }_{\mathrm{c}}$ & $\mathrm{q}_{\mathrm{p}}[\mathrm{ksf}]$ & Qp [kips] & $\alpha$ & $\mathrm{fs}[\mathrm{ksf}]$ & $\Sigma \mathrm{Qs}[\mathrm{kips}]$ & Qu [kips] & Qa [kips] & Qa (uplift) \\
\hline 0 & 0 & 0 & 0 & 0 & 0 & 0 & 0 & 0 & 0 & 0 \\
\hline 20 & 0.263 & 6.50 & 1.710 & 5.372 & 0.55 & 0.073 & 5.963 & 11.335 & 5.668 & 1.988 \\
\hline 40 & 0.526 & 6.58 & 3.460 & 10.870 & 0.55 & 0.145 & 30.065 & 40.935 & 20.468 & 10.022 \\
\hline 60 & 0.789 & 7.37 & 5.813 & 18.262 & 0.55 & 0.217 & 72.263 & 90.525 & 45.263 & 24.088 \\
\hline 80 & 1.052 & 8.05 & 8.471 & 26.612 & 0.55 & 0.289 & 132.556 & 159.168 & 79.584 & 44.185 \\
\hline 100 & 1.315 & 8.32 & 10.934 & 34.350 & 0.55 & 0.362 & 211.530 & 245.880 & 122.940 & 70.510 \\
\hline 120 & 1.578 & 8.58 & 13.536 & 42.525 & 0.55 & 0.434 & 308.140 & 350.665 & 175.333 & 102.713 \\
\hline 140 & 1.841 & 8.84 & 16.276 & 51.133 & 0.55 & 0.507 & 423.681 & 474.814 & 237.407 & 141.227 \\
\hline 160 & 2.104 & 9.00 & 18.936 & 59.489 & 0.55 & 0.579 & 556.609 & 616.098 & 308.049 & 185.536 \\
\hline
\end{tabular}

Qa [kips] for extreme condition

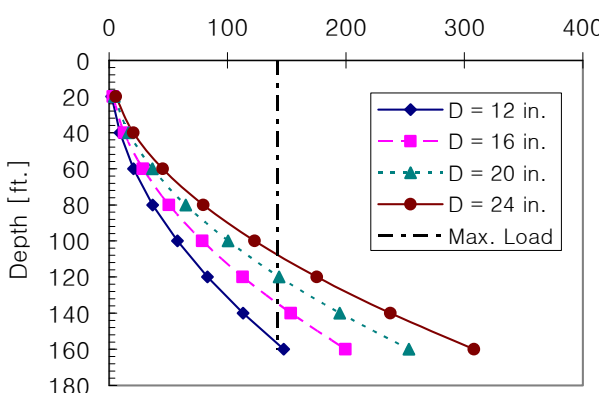

$\mathrm{Qa}$ (uplift) [kips] for extreme condition

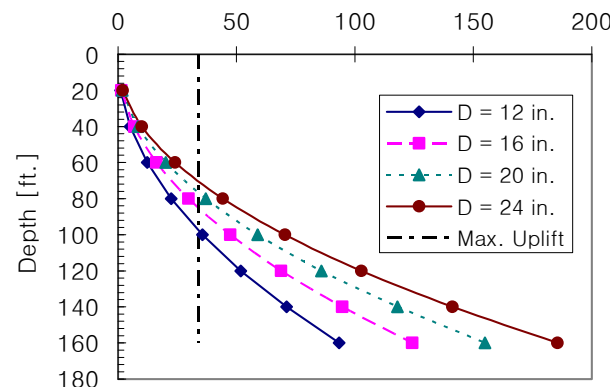




\section{APPENDIX B}

INPUT PARAMETERS USED IN THE CASE STUDY, SIX

SUGGESTED SYSTEMS FOR THE CAST STUDY, AND

KNAPSACK OPTIMIZATION ROUTINE 
- INPUT VALUES

\begin{tabular}{|c|c|c|c|c|c|c|c|c|c|c|c|}
\hline \multirow[b]{2}{*}{ Rig type } & \multirow[b]{2}{*}{ Rig name } & \multirow[b]{2}{*}{$\begin{array}{l}\text { Weight } \\
\text { (lb) }\end{array}$} & \multirow[b]{2}{*}{$\begin{array}{c}\text { Drilling } \\
\text { time (days) }\end{array}$} & \multirow[b]{2}{*}{$\begin{array}{c}\text { Move/rig up } \\
\text { (days) }\end{array}$} & \multirow[b]{2}{*}{$\begin{array}{c}\text { Rent/day } \\
(\$)\end{array}$} & \multirow[b]{2}{*}{$\begin{array}{c}\text { Footprint } \\
\text { (acres) }\end{array}$} & \multirow[b]{2}{*}{$\begin{array}{c}\text { Diesel } \\
\text { (gal/day) }\end{array}$} & \multirow[b]{2}{*}{$\left|\begin{array}{c}\text { Natural gas } \\
\text { (gal/day) }\end{array}\right|$} & \multicolumn{3}{|c|}{ Site Preparation } \\
\hline & & & & & & & & & $\begin{array}{c}\text { Gravels } \\
(\$)\end{array}$ & $\begin{array}{c}\text { Mats } \\
(\$)\end{array}$ & $\begin{array}{c}\text { Modules +piles } \\
(\$)\end{array}$ \\
\hline 1 & Traditional older vintage rig & $1,200,000$ & 12 & 4 & $\$ 12,500$ & 2 & 1680 & 2100 & $\$ 137,813$ & $\$ 122,500$ & $\$ 1,862,041$ \\
\hline 2 & Rapid Rig & 976,470 & 11 & 0.5 & $\$ 14,000$ & 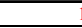 & 1200 & 1500 & $\$ 101,250$ & $\$ 90,000$ & $\$ 1,126,503$ \\
\hline 3 & LOC250 (CWD) & $1,048,000$ & 10 & 1 & $\$ 15,000$ & & 1200 & 1500 & $\$ 101,250$ & $\$ 90,000$ & $\$ 1,126,503$ \\
\hline
\end{tabular}

\begin{tabular}{|c|c|c|c|c|c|c|c|c|c|}
\hline \multirow[b]{2}{*}{ Rig type } & \multirow[b]{2}{*}{ Rig name } & \multicolumn{3}{|c|}{ Emissions } & \multicolumn{3}{|c|}{ Perceptions } & \multirow{2}{*}{$\begin{array}{l}\text { Safety } \\
\text { Value }\end{array}$} & \multirow{2}{*}{$\begin{array}{l}\text { How many } \\
\text { trucks? }\end{array}$} \\
\hline & & Air & $\begin{array}{l}\text { Solid\& } \\
\text { Liquid }\end{array}$ & $\begin{array}{l}\text { Noise } \\
\text { (TWA) }\end{array}$ & Gov. & Ind. & Public & & \\
\hline 1 & Traditional older vintage rig & 0.973 & & 78.630 & 0.500 & 1.000 & 0.500 & 0.500 & 20 \\
\hline 2 & Rapid Rig & 0.978 & & 77.020 & 1.000 & 0.500 & 1.000 & 1.000 & 16 \\
\hline 3 & LOC250 (CWD) & 0.977 & & 77.458 & 1.000 & 0.500 & 1.000 & 1.000 & 17 \\
\hline
\end{tabular}

\begin{tabular}{|c|c|c|c|c|c|c|c|c|c|c|}
\hline \multirow[b]{2}{*}{ Type } & \multirow[b]{2}{*}{ Engine name } & \multirow[b]{2}{*}{ Rent/day (\$) } & \multirow{2}{*}{\begin{tabular}{|c|} 
Ecological \\
Footprint \\
(Acres)
\end{tabular}} & \multicolumn{3}{|c|}{ Emissions } & \multicolumn{3}{|c|}{ Perceptions } & \multirow{2}{*}{$\begin{array}{l}\text { Safety } \\
\text { Value }\end{array}$} \\
\hline & & & & Air & $\begin{array}{l}\text { Solid\& } \\
\text { Liquid }\end{array}$ & $\begin{array}{l}\text { Noise } \\
\text { (TWA) }\end{array}$ & Gov. & Ind. & Public & \\
\hline \multirow[b]{2}{*}{1} & Internal combustion engine & $\$ 5,000$ & 0.011 & \begin{tabular}{l|l|l}
0.118 \\
\end{tabular} & & 110.073 & 0.500 & 1.000 & 0.500 & 0.750 \\
\hline & \begin{tabular}{|l|} 
Internal combustion engine $\mathrm{w} / \mathrm{SCR}$, \\
$\mathrm{w} /$ noise suppressor
\end{tabular} & $\$ 7,411$ & 0.011 & 0.431 & & 88.059 & 0.750 & 0.750 & 0.750 & 0.750 \\
\hline 2 & $\begin{array}{l}\text { Lean-burn natural gas engines w/noise } \\
\text { suppressor }\end{array}$ & $\$ 7,817$ & 0.005 & 0.878 & & 88.059 & 1.000 & 0.500 & 1.000 & 0.750 \\
\hline
\end{tabular}

\begin{tabular}{|c|c|c|c|c|c|c|c|c|c|c|c|}
\hline \multirow[b]{2}{*}{ Type } & \multirow[b]{2}{*}{ Drilling method } & \multirow[b]{2}{*}{$\begin{array}{c}\text { Drilling time } \\
\text { rate }\end{array}$} & \multirow[b]{2}{*}{$\begin{array}{c}\text { Rent/day } \\
(\$)\end{array}$} & \multirow{2}{*}{$\begin{array}{c}\text { Ecological } \\
\text { Footprint } \\
\text { (Acres) }\end{array}$} & \multicolumn{3}{|c|}{ Emissions } & \multicolumn{3}{|c|}{ Perceptions } & \multirow[b]{2}{*}{$\begin{array}{l}\text { Safety } \\
\text { Value }\end{array}$} \\
\hline & & & & & Air & $\begin{array}{l}\text { Solid\& } \\
\text { Liquid }\end{array}$ & Noise (TWA) & Gov. & Ind. & Public & \\
\hline 1 & Conventional overbalanced drilling & $100 \%$ & $\$ 17,000$ & & & & 116.700 & 1.000 & 0.500 & 0.500 & 0.500 \\
\hline 2 & $\begin{array}{l}\text { Underbalanced drilling w/noise } \\
\text { suppressor }\end{array}$ & $90 \%$ & $\$ 20,500$ & & & & 97.360 & 0.750 & 0.750 & 0.750 & 0.750 \\
\hline 3 & $\begin{array}{l}\text { Managed pressure drilling w/noise } \\
\text { suppressor }\end{array}$ & $90 \%$ & $\$ 21,500$ & & & & 95.760 & 0.750 & 0.750 & 1.000 & 1.000 \\
\hline
\end{tabular}

\begin{tabular}{|c|c|c|c|c|c|c|c|c|c|c|c|}
\hline \multirow[b]{2}{*}{ Type } & \multirow[b]{2}{*}{ Transportation type } & \multirow[b]{2}{*}{$\operatorname{Cost}(\$)$} & \multirow{2}{*}{\begin{tabular}{|c|} 
Ecological \\
Footprint \\
(Acres) \\
\end{tabular}} & \multicolumn{3}{|c|}{ Emissions } & \multicolumn{3}{|c|}{ Perceptions } & \multirow[b]{2}{*}{$\begin{array}{l}\text { Safety } \\
\text { Value }\end{array}$} & \multirow[b]{3}{*}{ Cost factor } \\
\hline & & & & Air & $\begin{array}{l}\text { Solid\& } \\
\text { Liquid }\end{array}$ & $\begin{array}{l}\text { Noise } \\
\text { (TWA) }\end{array}$ & Gov. & Ind. & Public & & \\
\hline 1 & Coventional diesel truck & $\$ 20,000$ & & & & & 0.250 & 1.000 & 0.250 & 0.750 & \\
\hline 2 & $\begin{array}{l}\text { Low sulphur diesel truck w/tier III } \\
\text { engine, w/noise suppressor }\end{array}$ & $\$ 28,000$ & & & & & 1.000 & 0.500 & 1.000 & 0.750 & 1.4 \\
\hline
\end{tabular}

\begin{tabular}{|c|c|c|c|c|c|c|c|c|c|c|c|c|}
\hline \multirow{2}{*}{ Rig types } & \multirow{2}{*}{ Transportation types } & \multirow{2}{*}{ Unit } & 0.2 & 0.4 & 0.4 & \multirow{2}{*}{$\begin{array}{c}\begin{array}{c}\text { Overall } \\
\text { score }\end{array} \\
\end{array}$} & \multirow{2}{*}{\begin{tabular}{|c} 
Operating hrs \\
1
\end{tabular}} & & \multirow{2}{*}{\multicolumn{2}{|c|}{ Noise for drilling site }} & \multirow{2}{*}{\multicolumn{2}{|c|}{$\begin{array}{c}\text { Suppressor factor } \\
20 \% \\
\end{array}$}} \\
\hline & & & $\mathrm{CO}$ & $\mathrm{NO}_{\mathrm{x}}$ & PM & & & & & & & \\
\hline \multirow{10}{*}{1} & \multirow{5}{*}{ Diesel truck } & (gram/hp-hr) & 15.5 & & 0.1 & \multirow{5}{*}{$\underline{0.973}$} & & & Noise (dBA/unit) & 76 & Total length (hr) & 5.0 \\
\hline & & (lb/hp-hr) & 0.03418 & 0.00882 & 0.00022 & & & & How many trucks & 4 & Ref. duration (hr) & 24.2 \\
\hline & & $(\mathrm{lb} / \mathrm{hr}) / \mathrm{unit}$ & 13.671 & 3.528 & 0.088 & & & & Noise (dBA) & 82.0 & Noise dose (\%) & 20.7 \\
\hline & & (lb/operating) & 273.420 & 70.560 & 1.764 & & $\mathrm{HP}$ & units & TWA & 78.6 & TWA w/suppressor & 62.9 \\
\hline & & U-value & 0.916 & 0.985 & 0.990 & & 400 & 20 & & & & \\
\hline & \multirow{5}{*}{ Low sulphur diesel truck w/tier III engir } & (gram/hp-hr) & 15.5 & 0.2 & 0.01 & \multirow{5}{*}{$\underline{0.982}$} & & & \begin{tabular}{|l|} 
Noise (dBA/unit) \\
\end{tabular} & 74 & Total length (hr) & 5.0 \\
\hline & & $(\mathrm{lb} / \mathrm{hp}-\mathrm{hr})$ & 0.03418 & 0.00044 & 0.00002 & & & & How many trucks & 4 & Ref. duration (hr) & 31.9 \\
\hline & & n $(\mathrm{lb} / \mathrm{hr} / \mathrm{unit}$ & 13.671 & 0.176 & 0.009 & & & & Noise (dBA) & 80.0 & Noise dose (\%) & 15.7 \\
\hline & & (lb/operating) & 273.420 & 3.528 & 0.176 & & $\mathrm{HP}$ & units & TWA & 76.6 & TWA w/suppressor & 61.3 \\
\hline & & U-value & 0.916 & 0.999 & 0.999 & & 400 & 20 & & & & \\
\hline \multirow{10}{*}{2} & \multirow{5}{*}{ Diesel truck } & (gram/hp-hr) & 15.5 & & 0.1 & \multirow{5}{*}{$\underline{0.978}$} & & & \begin{tabular}{|l|} 
Noise (dBA/unit) \\
\end{tabular} & 76 & Total length (hr) & 4.0 \\
\hline & & (lb/hp-hr) & 0.03418 & 0.00882 & 0.00022 & & & & How many trucks & 4 & Ref. duration (hr) & 24.2 \\
\hline & & $(\mathrm{lb} / \mathrm{hr}) / \mathrm{unit}$ & 13.671 & 3.528 & 0.088 & & & & Noise (dBA) & 82.0 & Noise dose $(\%)$ & 16.5 \\
\hline & & (lb/operating) & 218.736 & 56.448 & 1.411 & & $\mathrm{HP}$ & units & TWA & 77.0 & TWA w/suppressor & 61.6 \\
\hline & & U-value & 0.933 & 0.988 & 0.992 & & 400 & 16 & & & & \\
\hline & \multirow{5}{*}{ Low sulphur diesel truck w/tier III engir } & (gram/hp-hr) & 15.5 & 0.2 & 0.01 & \multirow{5}{*}{$\underline{0.986}$} & & & \begin{tabular}{|l|} 
Noise (dBA/unit) \\
\end{tabular} & 74 & Total length $(\mathrm{hr})$ & 4.0 \\
\hline & & (lb/hp-hr) & 0.03418 & 0.00044 & 0.00002 & & & & How many trucks & 4 & Ref. duration (hr) & 31.9 \\
\hline & & n $(\mathrm{lb} / \mathrm{hr}) / \mathrm{unit}$ & 13.671 & 0.176 & 0.009 & & & & Noise (dBA) & 80.0 & Noise dose $(\%)$ & 12.5 \\
\hline & & (lb/operating) & 218.736 & 2.822 & 0.141 & & $\mathrm{HP}$ & units & TWA & 75.0 & TWA w/suppressor & 60.0 \\
\hline & & U-value & 0.933 & 0.999 & 0.999 & & 400 & 16 & & & & \\
\hline \multirow{10}{*}{3} & \multirow{5}{*}{ Diesel truck } & (gram/hp-hr) & 15.5 & & 0.1 & \multirow{5}{*}{$\underline{0.977}$} & & & \begin{tabular}{|l|} 
Noise (dBA/unit) \\
\end{tabular} & 76 & Total length (hr) & 4.3 \\
\hline & & (lb/hp-hr) & 0.03418 & 0.00882 & 0.00022 & & & & How many trucks & 4 & Ref. duration (hr) & 24.2 \\
\hline & & $(\mathrm{lb} / \mathrm{hr}) / \mathrm{unit}$ & 13.671 & 3.528 & 0.088 & & & & Noise (dBA) & 82.0 & Noise dose (\%) & 17.6 \\
\hline & & (lb/operating) & 232.407 & 59.976 & 1.499 & & $\mathrm{HP}$ & units & TWA & 77.5 & TWA w/suppressor & 62.0 \\
\hline & & U-value & 0.928 & 0.987 & 0.991 & & 400 & 17 & & & & \\
\hline & \multirow{5}{*}{ Low sulphur diesel truck w/tier III engir } & (gram/hp-hr) & 15.5 & 0.2 & 0.01 & \multirow{5}{*}{$\underline{0.985}$} & & & \begin{tabular}{|l|} 
Noise (dBA/unit) \\
\end{tabular} & 74 & Total length (hr) & 4.3 \\
\hline & & $(\mathrm{lb} / \mathrm{hp}-\mathrm{hr})$ & 0.03418 & 0.00044 & 0.00002 & & & & How many trucks & 4 & Ref. duration (hr) & 31.9 \\
\hline & & n $(\mathrm{lb} / \mathrm{hr}) / \mathrm{unit}$ & 13.671 & 0.176 & 0.009 & & & & Noise (dBA) & 80.0 & Noise dose $(\%)$ & 13.3 \\
\hline & & (lb/operating) & 232.407 & 2.999 & 0.150 & & $\mathrm{HP}$ & units & TWA & 75.5 & TWA w/suppressor & 60.4 \\
\hline & & U-value & 0.928 & 0.999 & 0.999 & & 400 & 17 & & & & \\
\hline
\end{tabular}


- Emission of Road Construction (standard)

\begin{tabular}{|c|c|c|c|c|c|c|c|c|c|c|c|}
\hline \multirow{2}{*}{ Technologies } & \multirow{2}{*}{ Unit } & 0.2 & 0.4 & 0.4 & \multirow{2}{*}{$\begin{array}{l}\text { Overall } \\
\text { score }\end{array}$} & \multirow{2}{*}{$\begin{array}{c}\text { Operating hrs } \\
1\end{array}$} & & & & & \\
\hline & & $\mathrm{CO}$ & $\mathrm{NO}_{\mathrm{x}}$ & PM & & & & & & & \\
\hline \multirow{5}{*}{ Gravel: Diesel truck + dust } & (gram/hp-hr) & 15.5 & 4 & 0.1 & \multirow{5}{*}{$\underline{0.566}$} & & & Noise (dBA/unit) & 76 & Total length $(\mathrm{hr})$ & 79.3 \\
\hline & (lb/hp-hr) & 0.03418 & 0.00882 & 0.00022 & & & & How many trucks & 4 & Ref. duration (hr) & 24.2 \\
\hline & (lb/hr)/unit & 10.253 & 2.646 & 0.216 & & & & Noise (dBA) & 82.0 & Noise dose (\%) & 327.7 \\
\hline & (lb/operating) & 3250.280 & 838.782 & 68.520 & & $\mathrm{HP}$ & units & TWA & 98.6 & TWA w/suppressor & 78.8 \\
\hline & U-value & 0.000 & 0.822 & 0.593 & & 300 & 317 & & & & \\
\hline \multirow{5}{*}{ Mat: Diesel truck } & (gram/hp-hr) & 15.5 & 4 & 0.1 & \multirow{5}{*}{$\underline{0.964}$} & & & Noise (dBA/unit) & 76 & Total length $(\mathrm{hr})$ & 9.0 \\
\hline & (lb/hp-hr) & 0.03418 & 0.00882 & 0.00022 & & & & How many trucks & 4 & Ref. duration (hr) & 24.2 \\
\hline & (lb/hr)/unit & 10.253 & 2.646 & 0.066 & & & & Noise (dBA) & 82.0 & Noise dose (\%) & 37.2 \\
\hline & (lb/operating) & 369.117 & 95.256 & 2.381 & & $\mathrm{HP}$ & units & TWA & 82.9 & TWA w/suppressor & 66.3 \\
\hline & U-value & 0.886 & 0.980 & 0.986 & & 300 & 36 & & & & \\
\hline \multirow{5}{*}{$\begin{array}{c}\text { Gravel: Low sulphur diesel truck } \\
\text { w/tier III engine + dust }\end{array}$} & (gram/hp-hr) & 15.5 & 0.2 & 0.01 & \multirow{5}{*}{$\underline{0.679}$} & & & Noise (dBA/unit) & 74 & Total length $(\mathrm{hr})$ & 79.3 \\
\hline & (lb/hp-hr) & 0.03418 & 0.00044 & 0.00002 & & & & How many trucks & 4 & Ref. duration (hr) & 31.9 \\
\hline & $(\mathrm{lb} / \mathrm{hr}) / \mathrm{unit}$ & 10.253 & 0.132 & 0.157 & & & & Noise (dBA) & 80.0 & Noise dose (\%) & 248.4 \\
\hline & (lb/operating) & 3250.280 & 41.939 & 49.647 & & $\mathrm{HP}$ & units & TWA & 96.6 & TWA w/suppressor & 77.2 \\
\hline & U-value & 0.000 & 0.991 & 0.705 & & 300 & 317 & & & & \\
\hline \multirow{5}{*}{$\begin{array}{l}\text { Mat: Low sulphur diesel truck } \\
\text { w/tier III engine }\end{array}$} & (gram/hp-hr) & 15.5 & 0.2 & 0.01 & \multirow{5}{*}{$\underline{0.976}$} & & & Noise (dBA/unit) & 74 & Total length $(\mathrm{hr})$ & 9.0 \\
\hline & $(\mathrm{lb} / \mathrm{hp}-\mathrm{hr})$ & 0.03418 & 0.00044 & 0.00002 & & & & How many trucks & 4 & Ref. duration (hr) & 31.9 \\
\hline & (lb/hr)/unit & 10.253 & 0.132 & 0.007 & & & & Noise (dBA) & 80.0 & Noise dose (\%) & 28.2 \\
\hline & (lb/operating) & 369.117 & 4.763 & 0.238 & & HP & units & TWA & \begin{tabular}{l|l}
80.9 \\
\end{tabular} & TWA w/suppressor & 64.7 \\
\hline & U-value & 0.886 & 0.999 & 0.999 & & 300 & 36 & & & & \\
\hline
\end{tabular}

- Emission of Site Preparation (standard)

\begin{tabular}{|c|c|c|c|c|c|c|c|c|c|c|c|c|}
\hline \multirow{2}{*}{ Rig types } & \multirow{2}{*}{ Technologies } & \multirow{2}{*}{ Unit } & 0.2 & 0.4 & 0.4 & \multirow{2}{*}{$\begin{array}{c}\text { Overall } \\
\text { score }\end{array}$} & \multirow{2}{*}{\begin{tabular}{|c} 
Operating hrs \\
1
\end{tabular}} & & & & & \\
\hline & & & $\mathrm{CO}$ & $\mathrm{NO}_{\mathrm{x}}$ & PM & & & & & & & \\
\hline \multirow{30}{*}{1} & \multirow{5}{*}{ Gravel: Diesel truck + dust } & (gram/hp-hr) & 15.5 & & 0.1 & \multirow{5}{*}{$\underline{0.598}$} & & & \begin{tabular}{|l|} 
Noise (dBA/unit) \\
\end{tabular} & 76 & Total length (hr) & 73.5 \\
\hline & & (lb/hp-hr) & 0.03418 & 0.00882 & 0.00022 & & & & How many trucks & 4 & \begin{tabular}{l|l} 
Ref. duration (hr) \\
\end{tabular} & 24.2 \\
\hline & & $(\mathrm{lb} / \mathrm{hr}) / \mathrm{unit}$ & 10.253 & 2.646 & 0.216 & & & & Noise (dBA) & 82.0 & Noise dose (\%) & 303.9 \\
\hline & & (lb/operating) & 3014.456 & 777.924 & 63.548 & & $\mathrm{HP}$ & units & TWA & 98.0 & TWA w/suppressor & 78.4 \\
\hline & & U-value & 0.073 & 0.835 & 0.623 & & 300 & 294 & & & & \\
\hline & \multirow{5}{*}{ Mat: Diesel truck } & (gram/hp-hr) & 15.5 & 4 & 0.1 & \multirow{5}{*}{$\underline{0.967}$} & & & \begin{tabular}{|l|} 
Noise (dBA/unit) \\
\end{tabular} & 76 & Total length $(\mathrm{hr})$ & 8.3 \\
\hline & & (lb/hp-hr) & 0.03418 & 0.00882 & 0.00022 & & & & How many trucks & 4 & $\begin{array}{ll}\text { Ref. duration (hr) } \\
\end{array}$ & 24.2 \\
\hline & & $(\mathrm{lb} / \mathrm{hr}) / \mathrm{unit}$ & 10.253 & 2.646 & 0.066 & & & & Noise (dBA) & 82.0 & Noise dose (\%) & 34.1 \\
\hline & & (lb/operating) & 338.357 & 87.318 & 2.183 & & $\mathrm{HP}$ & units & TWA & 82.2 & TWA w/suppressor & 65.8 \\
\hline & & U-value & 0.896 & 0.981 & 0.987 & & 300 & 33 & & & & \\
\hline & \multirow{5}{*}{ Module: Diesel truck + hammer } & (gram/hp-hr) & 15.5 & 7 & 0.1 & \multirow{5}{*}{$\underline{0.973}$} & & & \begin{tabular}{|l|} 
Noise (dBA/unit) \\
\end{tabular} & 76 & Total length $(\mathrm{hr})$ & 5.0 \\
\hline & & $(\mathrm{lb} / \mathrm{hp}-\mathrm{hr})$ & 0.03418 & 0.00882 & 0.00022 & & & & How many trucks & 4 & $\begin{array}{ll}\text { Ref. duration (hr) } \\
\end{array}$ & 24.2 \\
\hline & & $(\mathrm{lb} / \mathrm{hr}) / \mathrm{unit}$ & $\begin{array}{ll}10.253 \\
\end{array}$ & 2.646 & 0.066 & & & & Noise (dBA) & 82.0 & Noise dose $(\%)$ & 287.3 \\
\hline & & (lb/operating) & 270.686 & 69.854 & 1.746 & & HP & units & TWA & 97.6 & TWA w/suppressor & 78.1 \\
\hline & & U-value & 0.917 & 0.985 & 0.990 & & 300 & 20 & & & & \\
\hline & & (gram/hp-hr) & 15.5 & 0.2 & 0.01 & & & & \begin{tabular}{|l|} 
Noise (dBA/unit) \\
\end{tabular} & 74 & Total length (hr) & 73.5 \\
\hline & & (lb/hp-hr) & 0.03418 & 0.00044 & 0.00002 & & & & How many trucks & 4 & Ref. duration (hr) & 31.9 \\
\hline & Gravel: Low sulphur diesel truck & $(\mathrm{lb} / \mathrm{hr}) /$ unit & 10.253 & 0.132 & 0.157 & $\underline{0.702}$ & & & Noise (dBA) & 80.0 & Noise dose (\%) & 230.3 \\
\hline & & (lb/operating) & 3014.456 & 38.896 & 46.045 & & $\mathrm{HP}$ & units & TWA & 96.0 & TWA w/suppressor & 76.8 \\
\hline & & U-value & 0.073 & 0.992 & 0.727 & & 300 & 294 & & & & \\
\hline & & (gram/hp-hr) & 15.5 & 0.2 & 0.01 & & & & \begin{tabular}{|l|} 
Noise (dBA/unit) \\
\end{tabular} & 74 & Total length (hr) & 8.3 \\
\hline & & (lb/hp-hr) & 0.03418 & 0.00044 & 0.00002 & & & & \begin{tabular}{|l|} 
How many trucks \\
\end{tabular} & 4 & Ref. duration (hr) & 31.9 \\
\hline & Mat: Low sulphur diesel truck & $(\mathrm{lb} / \mathrm{hr}) / \mathrm{unit}$ & 10.253 & 0.132 & 0.007 & 0.978 & & & Noise (dBA) & 80.0 & Noise dose (\%) & 25.9 \\
\hline & & (lb/operating) & 338.357 & 4.366 & 0.218 & & HP & units & TWA & 80.2 & TWA w/suppressor & 64.2 \\
\hline & & U-value & 0.896 & 0.999 & 0.999 & & 300 & 33 & & & & \\
\hline & & (gram/hp-hr) & 15.5 & 0.2 & 0.01 & & & & \begin{tabular}{|l|} 
Noise (dBA/unit) \\
\end{tabular} & 74 & Total length (hr) & 5.0 \\
\hline & & (lb/hp-hr) & 0.03418 & 0.00044 & 0.00002 & & & & How many trucks & 4 & Ref. duration (hr) & 31.9 \\
\hline & Module: Low sulphur diesel truck & $(\mathrm{lb} / \mathrm{hr}) / \mathrm{unit}$ & 10.253 & 0.132 & 0.007 & $\underline{0.983}$ & & & Noise (dBA) & 80.0 & Noise dose (\%) & 282.3 \\
\hline & & (lb/operating) & 270.686 & 3.493 & 0.175 & & $\mathrm{HP}$ & units & TWA & 97.5 & TWA w/suppressor & 78.0 \\
\hline & & U-value & 0.917 & 0.999 & 0.999 & & 300 & 20 & & & & \\
\hline & & (gram/hp-hr) & 15.5 & & 0.1 & & & & \begin{tabular}{|l|} 
Noise (dBA/unit) \\
\end{tabular} & 76 & Total length $(\mathrm{hr})$ & 54.0 \\
\hline & & (lb/hp-hr) & 0.03418 & 0.00882 & 0.00022 & & & & How many trucks & 4 & Ref. duration (hr) & 24.2 \\
\hline & Gravel: Diesel truck + dust & $(\mathrm{lb} / \mathrm{hr}) / \mathrm{unit}$ & 10.253 & 2.646 & 0.216 & $\underline{0.704}$ & & & Noise (dBA) & 82.0 & Noise dose $(\%)$ & 223.3 \\
\hline & & (lb/operating) & 2214.702 & 571.536 & 46.688 & & $\mathrm{HP}$ & units & TWA & 95.8 & TWA w/suppressor & 76.6 \\
\hline & & U-value & 0.319 & 0.879 & 0.723 & & 300 & 216 & & & & \\
\hline & & (gram/hp-hr) & 15.5 & & 0.1 & & & & \begin{tabular}{|l|} 
Noise (dBA/unit) \\
\end{tabular} & 76 & Total length (hr) & 6.0 \\
\hline & & $(\mathrm{lb} / \mathrm{hp}-\mathrm{hr})$ & 0.03418 & 0.00882 & 0.00022 & & & & How many trucks & 4 & Ref. duration (hr) & 24.2 \\
\hline & Mat: Diesel truck & $(\mathrm{lb} / \mathrm{hr}) / \mathrm{unit}$ & 10.253 & 2.646 & 0.066 & $\underline{0.976}$ & & & Noise (dBA) & 82.0 & Noise dose $(\%)$ & 24.8 \\
\hline & & (lb/operating) & 246.078 & 63.504 & 1.588 & & HP & units & TWA & 79.9 & TWA w/suppressor & 64.0 \\
\hline & & U-value & 0.924 & 0.987 & 0.991 & & 300 & 24 & & & & \\
\hline & & (gram/hp-hr) & 15.5 & & 0.1 & & & & \begin{tabular}{|l|} 
Noise (dBA/unit) \\
\end{tabular} & 76 & Total length (hr) & 3.0 \\
\hline & & (lb/hp-hr) & 0.03418 & 0.00882 & 0.00022 & & & & How many trucks & 4 & Ref. duration (hr) & 24.2 \\
\hline & Module: Diesel truck + hammer & $(\mathrm{lb} / \mathrm{hr}) / \mathrm{unit}$ & 10.253 & 2.646 & 0.066 & $\underline{0.983}$ & & & Noise (dBA) & 82.0 & Noise dose $(\%)$ & 212.4 \\
\hline & & (lb/operating) & 172.255 & 44.453 & 1.111 & & $\mathrm{HP}$ & units & TWA & 95.4 & TWA w/suppressor & 76.3 \\
\hline 283 & & U-value & 0.947 & 0.991 & 0.993 & & 300 & 12 & & & & \\
\hline $2 \& 3$ & & (gram/hp-hr) & 15.5 & 0.2 & 0.01 & & & & \begin{tabular}{|l|} 
Noise (dBA/unit) \\
\end{tabular} & 74 & Total length (hr) & 54.0 \\
\hline & & (lb/hp-hr) & 0.03418 & 0.00044 & 0.00002 & & & & How many trucks & 4 & Ref. duration (hr) & 31.9 \\
\hline & Gravel: Low sulphur diesel truck & $(\mathrm{lb} / \mathrm{hr} / \mathrm{unit}$ & 10.253 & 0.132 & 0.157 & $\underline{0.781}$ & & & Noise (dBA) & 80.0 & Noise dose (\%) & 169.2 \\
\hline & & (lb/operating) & 2214.702 & 28.577 & 33.829 & & $\mathrm{HP}$ & units & TWA & 93.8 & \begin{tabular}{|l|} 
TWA w/suppressor \\
\end{tabular} & 75.0 \\
\hline & & U-value & 0.319 & 0.994 & 0.799 & & 300 & 216 & & & & \\
\hline & & (gram/hp-hr) & 15.5 & 0.2 & 0.01 & & & & Noise (dBA/unit) & 74 & Total length $(\mathrm{hr})$ & 6.0 \\
\hline & & (lb/hp-hr) & 0.03418 & 0.00044 & 0.00002 & & & & How many trucks & 4 & Ref. duration (hr) & 31.9 \\
\hline & Mat: Low sulphur diesel truck & $(\mathrm{lb} / \mathrm{hr}) / \mathrm{unit}$ & 10.253 & 0.132 & 0.007 & $\underline{0.984}$ & & & Noise (dBA) & 80.0 & Noise dose (\%) & 18.8 \\
\hline & & (lb/operating) & 246.078 & 3.175 & 0.159 & & $\mathrm{HP}$ & units & TWA & 77.9 & \begin{tabular}{|l|} 
TWA w/suppressor \\
\end{tabular} & 62.4 \\
\hline & & U-value & 0.924 & 0.999 & 0.999 & & 300 & 24 & & & & \\
\hline & & (gram/hp-hr) & 15.5 & 0.2 & 0.01 & & & & \begin{tabular}{|l|} 
Noise (dBA/unit) \\
\end{tabular} & 74 & Total length (hr) & 3.0 \\
\hline & & $\begin{array}{l}\mathrm{l} / \mathrm{b} / \mathrm{hp}-\mathrm{hr}) \\
\end{array}$ & 0.03418 & 0.00044 & 0.00002 & & & & How many trucks & 4 & Ref. duration (hr) & 31.9 \\
\hline & Module: Low sulphur diesel truck & $(\mathrm{lb} / \mathrm{hr}) /$ unit & 10.253 & 0.132 & 0.007 & $\underline{0.989}$ & & & Noise (dBA) & 80.0 & Noise dose (\%) & 209.4 \\
\hline & & (lb/operating) & 172.255 & 2.223 & 0.111 & & HP & units & TWA & 95.3 & \begin{tabular}{|l|} 
TWA w/suppressor \\
\end{tabular} & 76.3 \\
\hline & & U-value & $\begin{array}{ll}0.947 \\
\end{array}$ & 1.000 & 0.999 & & 300 & 12 & & & & \\
\hline
\end{tabular}




\begin{tabular}{|c|c|c|c|c|c|c|c|c|c|c|c|c|}
\hline & \multirow{2}{*}{ Engine types } & \multirow{2}{*}{ Unit } & 0.2 & 0.4 & 0.4 & \multirow{2}{*}{$\begin{array}{c}\text { Overall } \\
\text { score }\end{array}$} & & & & & & \\
\hline & & & $\mathrm{CO}$ & $\mathrm{NO}_{\mathrm{x}}$ & PM & & & & & & & \\
\hline \multirow{15}{*}{\begin{tabular}{|c|} 
Proportion \\
$100 \%$ \\
\end{tabular}} & \multirow{5}{*}{ Internal Combustion Engine } & $(\mathrm{lb} / \mathrm{MWh})$ & 6.2 & 21.8 & 0.78 & \multirow{5}{*}{$\underline{0.118}$} & \begin{tabular}{|l|} 
Power (MW) \\
\end{tabular} & units & \begin{tabular}{|l|} 
Noise (dBA//unit) \\
\end{tabular} & 86 & Total length (hr) & 144.0 \\
\hline & & $(\mathrm{lb} / \mathrm{hr}) /$ unit & 6.200 & 21.800 & 0.780 & & & & \begin{tabular}{|l|} 
How many engines \\
\end{tabular} & & Ref. duration (hr) & 13.4 \\
\hline & & & 6.200 & 21.800 & 0.780 & & & & \begin{tabular}{|l|} 
Noise $(\mathrm{dBA})$ \\
\end{tabular} & 86.3 & Noise dose (\%) & 1616.3 \\
\hline & & (lb/operating) & 1339.200 & 4708.800 & 168.480 & & & & TWA & 110.1 & TWA w/suppressor & 88.1 \\
\hline & & 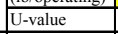 & 0.588 & 0.000 & 0.000 & & & & & & & \\
\hline & \multirow{5}{*}{ Internal Combustion Engine with SCR } & $(\mathrm{lb} / \mathrm{MWh})$ & 6.2 & 4.7 & 0.78 & \multirow{5}{*}{$\underline{0.431}$} & & & \begin{tabular}{|l|} 
Noise (dBA/unit) \\
\end{tabular} & 86 & $\begin{array}{l}\text { Total length }(\mathrm{hr}) \\
\end{array}$ & 144.0 \\
\hline & & $(\mathrm{lb} / \mathrm{hr}) / \mathrm{unit}$ & 6.200 & 4.700 & 0.780 & & & & \begin{tabular}{|l|} 
How many engines \\
\end{tabular} & & Ref. duration $(\mathrm{hr})$ & 13.4 \\
\hline & & $(\mathrm{lb} / \mathrm{hr})^{*}$ portion & 6.200 & 4.700 & 0.780 & & & & Noise (dBA) & 86.3 & Noise dose (\%) & 1616.3 \\
\hline & & \begin{tabular}{|l|} 
(lb/operating) \\
\end{tabular} & 1339.200 & 1015.200 & 168.480 & & & & TWA & 110.1 & TWA w/suppressor & 88.1 \\
\hline & & \begin{tabular}{|l|} 
U-value \\
\end{tabular} & 0.588 & 0.784 & 0.000 & & & & & & & \\
\hline & \multirow{5}{*}{ Lean-burn natural gas engine (SI) } & $(\mathrm{lb} / \mathrm{MWh})$ & & 2.2 & 0.03 & \multirow{5}{*}{$\underline{0.878}$} & & & \begin{tabular}{|l|} 
Noise (dBA/unit) \\
\end{tabular} & 86 & $\begin{array}{l}\text { Total length (hr) } \\
\end{array}$ & 144.0 \\
\hline & & $(\mathrm{lb} / \mathrm{hr}) / \mathrm{unit}$ & 5.000 & 2.200 & 0.030 & & & & \begin{tabular}{|l|} 
How many engines \\
\end{tabular} & & Ref. duration $(\mathrm{hr})$ & 13.4 \\
\hline & & \begin{tabular}{|l|}
$(\mathrm{lb} / \mathrm{hr})^{*}$ portion \\
\end{tabular} & 5.000 & 2.200 & 0.030 & & & & Noise (dBA) & 86.3 & Noise dose (\%) & 1616.3 \\
\hline & & \begin{tabular}{|l|} 
(lb/operating) \\
\end{tabular} & 1080.000 & 475.200 & 6.480 & & & & TWA & 110.1 & TWA w/suppressor & 88.1 \\
\hline & & U-value & 0.668 & 0.899 & 0.962 & & & & & & & \\
\hline \multirow{19}{*}{\begin{tabular}{|c} 
Proportion \\
$0 \%$ \\
\end{tabular}} & \multirow{5}{*}{ Power from grid } & $(\mathrm{lb} / \mathrm{MWh})$ & & 0 & & \multirow{5}{*}{1.000} & \begin{tabular}{|l|} 
Power (MW) \\
\end{tabular} & units & \begin{tabular}{|l|} 
Noise (dBA/unit) \\
\end{tabular} & & Total length (hr) & 144.0 \\
\hline & & $\begin{array}{ll}(1 / b / h r / / n n i t \\
\end{array}$ & 0.000 & 0.000 & 0.000 & & \begin{tabular}{|l|l}
1 \\
1
\end{tabular} & $\frac{1}{1}$ & \begin{tabular}{|l|} 
How many engines \\
\end{tabular} & & Ref. duration (hr) & 2097152.0 \\
\hline & & (lb/hr) $)^{*}$ portion & 0.000 & 0.000 & 0.000 & & & & \begin{tabular}{|c|} 
Noise (dBA) \\
\end{tabular} & 0.0 & Noise dose ( $(\%)$ & 0.0 \\
\hline & & \begin{tabular}{|l|} 
(lb/operating) \\
\end{tabular} & 0.000 & 0.000 & 0.000 & & & & TWA & 0.0 & $\begin{array}{l}\text { TWA w/suppressor } \\
\end{array}$ & \\
\hline & & \begin{tabular}{|l|}
$\mathrm{U}$-value \\
\end{tabular} & 1.000 & 1.000 & 1.000 & & & & & & & \\
\hline & & & & & & \multicolumn{3}{|c|}{ - Drill Noise } & & & & \\
\hline & & & & & & \multirow{4}{*}{\multicolumn{3}{|c|}{ Overbalanced drilling $(\mathrm{OB})$}} & \begin{tabular}{|l|} 
Noise (dBA/unit) \\
\end{tabular} & 95 & Total length $(\mathrm{hr} r)$ & 144.0 \\
\hline & & & & & & & & & \begin{tabular}{|l|} 
How many drills \\
\end{tabular} & & Ref. duration (hr) & 4.0 \\
\hline & & & & & & & & & Noise (dBA) & 95.0 & Noise dose (\%) & 4050.0 \\
\hline & & & & & & & & & TWA & 116.7 & TWA w/suppressor & 93.4 \\
\hline & & & & & & \multirow{4}{*}{\multicolumn{3}{|c|}{ Underbalanced drilling (UB) }} & Noise (dBA/unit) & 100 & Total length $(\mathrm{hr})$ & 144.0 \\
\hline & & & & & & & & & \begin{tabular}{|l|} 
How many drills \\
\end{tabular} & & Ref. duration (hr) & \\
\hline & & & & & & & & & \begin{tabular}{|r|} 
Noise (dBA) \\
\end{tabular} & 100.0 & Noise dose (\%) & 8100.0 \\
\hline & & & & & & & & & TWA & 121.7 & TWA w/suppressor & 97.4 \\
\hline & & & & & & \multirow{5}{*}{\multicolumn{3}{|c|}{ Managed pressure drilling }} & & & & \\
\hline & & & & & & & & & \begin{tabular}{|l|} 
Noise (dBA/unit) \\
\end{tabular} & 98 & Total length $(\mathrm{hr})$ & 144.0 \\
\hline & & & & & & & & & \begin{tabular}{|l|} 
How many drills \\
\end{tabular} & & $\begin{array}{ll}\text { Ref. duration }(\mathrm{hr}) \\
\end{array}$ & 2.6 \\
\hline & & & & & & & & & Noise (dBA) & 98.0 & Noise dose (\%) & 6138.7 \\
\hline & & & & & & & & & TWA & 119.7 & TWA w/suppressor & 95.8 \\
\hline
\end{tabular}

[When 'Traditional older vintage rig' is selected]

\begin{tabular}{|c|c|c|c|c|c|c|c|c|c|c|c|c|c|c|}
\hline \multirow{2}{*}{$\begin{array}{l}\text { Sub- } \\
\text { sets }\end{array}$} & \multirow[b]{2}{*}{ Technologies } & \multirow[b]{2}{*}{ Buy(\$) } & \multirow{2}{*}{$\begin{array}{c}\text { Resale } \\
\text { Value (\$) }\end{array}$} & \multirow{2}{*}{$\begin{array}{c}\text { Rent /day } \\
\text { (\$) }\end{array}$} & \multirow{2}{*}{\begin{tabular}{|c} 
Daily \\
Rate (\$)
\end{tabular}} & \multirow{2}{*}{$\begin{array}{c}\text { Total cost } \\
\text { (\$) }\end{array}$} & \multirow{2}{*}{$\begin{array}{c}\text { Ecological } \\
\text { Footprint } \\
\text { (Acres) }\end{array}$} & \multicolumn{3}{|c|}{ Emissions } & \multicolumn{3}{|c|}{ Perceptions } & \multirow{2}{*}{$\begin{array}{l}\text { Safety } \\
\text { Value }\end{array}$} \\
\hline & & & & & & & & Air & $\begin{array}{l}\text { Solid\& } \\
\text { Liquid }\end{array}$ & \begin{tabular}{l|} 
Noise \\
(TWA $)$
\end{tabular} & Gov. & Ind. & Public & \\
\hline 1 & Coventional diesel truck & & & & & & & & & & 0.250 & 1.000 & 0.250 & 0.750 \\
\hline \multirow{3}{*}{2} & Gravel roads & $\$ 148,500$ & $\$ 0$ & & $\$ 9,281$ & $\$ 148,500$ & 3.030 & 0.566 & & 98.562 & 0.250 & 1.000 & 0.250 & 0.500 \\
\hline & DURA-BASE from Composite Mat (buy) & $\$ 2,706,000$ & $\$ 2,164,800$ & & $\$ 33,825$ & $\$ 541,200$ & 1.515 & 0.964 & & 82.870 & 1.000 & 0.500 & 1.000 & 1.000 \\
\hline & DURA-BASE from Composite Mat (rent) & & & & & $\$ 132,000$ & 1.515 & 0.964 & & 82.870 & 1.000 & 0.500 & 1.000 & 1.000 \\
\hline \multirow{4}{*}{3} & Gravel pad & $\$ 137,813$ & $\$ 0$ & & $\$ 8,613$ & $\$ 137,813$ & 2.812 & 0.598 & & 98.019 & 0.250 & 1.000 & 0.250 & 0.500 \\
\hline & DURA-BASE from Composite Mat (buy) & $\$ 2,511,250$ & $\$ 2,009,000$ & & $\$ 31,391$ & $\$ 502,250$ & 1.406 & 0.967 & & 82.242 & 0.750 & 0.750 & 0.750 & 1.000 \\
\hline & DURA-BASE from Composite Mat (rent) & & & & & $\$ 122,500$ & 1.406 & 0.967 & & 82.242 & 0.750 & 0.750 & 0.750 & 1.000 \\
\hline & Aluminum modules + driven piles & $\$ 1,862,041$ & $\$ 1,489,633$ & & $\$ 23,276$ & $\$ 372,408$ & 0.007 & 0.973 & & 97.614 & 1.000 & 0.500 & 1.000 & 0.500 \\
\hline 4 & Traditional older vintage rig & & & $\$ 12,500$ & $\$ 12,500$ & $\$ 220,000$ & & 0.973 & & 78.630 & 0.500 & 1.000 & 0.500 & 0.500 \\
\hline \multirow{3}{*}{5} & Internal combustion engine & & & $\$ 5,000$ & $\$ 5,000$ & $\$ 80,000$ & & 0.118 & & 110.073 & 0.500 & 1.000 & 0.500 & 0.750 \\
\hline & \multicolumn{2}{|l|}{ Internal combustion engine $\mathrm{w} / \mathrm{SCR}, \mathrm{w} /$ noise suppressor } & & $\$ 7,411$ & $\$ 7,411$ & $\$ 118,569$ & & 0.431 & & 88.059 & 0.750 & 0.750 & 0.750 & 0.750 \\
\hline & \multicolumn{2}{|l|}{ Lean-burn natural gas engines w/noise suppressor } & & $\$ 7,817$ & $\$ 7,817$ & $\$ 125,073$ & & 0.878 & & 88.059 & 1.000 & 0.500 & 1.000 & 0.750 \\
\hline \multirow{3}{*}{6} & Conventional diesel & & & $\$ 6,720$ & $\$ 6,720$ & $\$ 94,080$ & & & & & 0.500 & 1.000 & 0.500 & 0.500 \\
\hline & Low sulphur diesel & & & $\$ 7,056$ & $\$ 7,056$ & $\$ 98,784$ & & & & & 0.750 & 0.750 & 1.000 & 0.750 \\
\hline & Natural gas & & & $\$ 4,200$ & $\$ 4,200$ & $\$ 58,800$ & & & & & 1.000 & 0.500 & 1.000 & 0.750 \\
\hline 7 & N/A & & & $\$ 0$ & so & $\$ 0$ & 0.000 & 1.000 & & 0.000 & 0.250 & 1.000 & 0.250 & 1.000 \\
\hline 8 & N/A & $\$ 0$ & $\$ 0$ & & $\$ 0$ & $\$ 0$ & 0.000 & & & & 0.250 & 1.000 & 0.250 & 1.000 \\
\hline 9 & Conventional overbalanced drilling & & & $\$ 17,000$ & $\$ 17,000$ & $\$ 204,000$ & & & & 116.700 & 1.000 & 0.500 & 0.500 & 0.500 \\
\hline 10 & Water-based muds & & & & & $\$ 47,940$ & & & & & 1.000 & 1.000 & 1.000 & 1.000 \\
\hline \multirow{2}{*}{11} & Lined reserve pit + solid control equip.* & & & $\$ 2,000$ & $\$ 2,000$ & $\$ 24,000$ & 0.037 & & 0.500 & & 0.750 & 0.750 & 0.750 & 0.500 \\
\hline & Closed loop + containers + solid control equip.* & & & $\$ 3,000$ & $\$ 3,000$ & $\$ 36,000$ & 0.000 & & 1.000 & & 1.000 & 0.500 & 1.000 & 0.750 \\
\hline \multirow{2}{*}{12} & Cuttings injection & & & $\$ 5,000$ & $\$ 5,000$ & $\$ 60,000$ & & & 1.000 & & 1.000 & 0.500 & 1.000 & 0.750 \\
\hline & Chemical fixation and solidification (CFS) & & & & & $\$ 61,710$ & & & 0.250 & & 0.750 & 0.750 & 1.000 & 0.500 \\
\hline \begin{tabular}{|c|}
13 \\
\end{tabular} & $\mathrm{~N} / \mathrm{A}$ & & & & & & & & & & & & & \\
\hline
\end{tabular}


[When 'Rapid Rig' is selected]

\begin{tabular}{|c|c|c|c|c|c|c|c|c|c|c|c|c|c|c|}
\hline \multirow{2}{*}{$\begin{array}{l}\text { Sub- } \\
\text { sets }\end{array}$} & \multirow[b]{2}{*}{ Technologies } & \multirow[b]{2}{*}{ Buy(\$) } & \multirow{2}{*}{$\begin{array}{c}\text { Resale } \\
\text { Value (\$) }\end{array}$} & \multirow{2}{*}{$\begin{array}{c}\text { Rent } / \text { day } \\
\text { (\$) }\end{array}$} & \multirow{2}{*}{\begin{tabular}{|c|} 
Daily \\
Rate (\$)
\end{tabular}} & \multirow{2}{*}{$\begin{array}{c}\text { Total cost } \\
\text { (\$) }\end{array}$} & \multirow{2}{*}{$\begin{array}{c}\text { Ecological } \\
\text { Footprint } \\
\text { (Acres) }\end{array}$} & \multicolumn{3}{|c|}{ Emissions } & \multicolumn{3}{|c|}{ Perceptions } & \multirow{2}{*}{$\begin{array}{l}\text { Safety } \\
\text { Value }\end{array}$} \\
\hline & & & & & & & & Air & $\begin{array}{l}\text { Solid\& } \\
\text { Liquid }\end{array}$ & $\begin{array}{l}\text { Noise } \\
\text { (TWA })\end{array}$ & Gov. & Ind. & Public & \\
\hline 1 & \multicolumn{2}{|c|}{ Low sulphur diesel truck w/tier III engine, w/noise suppressor } & & & & & & & & & 1.000 & 0.500 & 1.000 & 0.750 \\
\hline \multirow{3}{*}{2} & Gravel roads & $\$ 198,396$ & $\$ 0$ & & $\$ 19,077$ & $\$ 198,396$ & 3.030 & 0.679 & & 77.250 & 0.500 & 0.750 & 0.500 & 0.500 \\
\hline & DURA-BASE from Composite Mat (buy) & $\$ 2,721,840$ & $\$ 2,177,472$ & & $\$ 52,343$ & $\$ 544,368$ & 1.515 & 0.976 & & 64.696 & 1.000 & 0.500 & 1.000 & 1.000 \\
\hline & DURA-BASE from Composite Mat (rent) & & & & & $\$ 147,840$ & 1.515 & 0.976 & & 64.696 & 1.000 & 0.500 & 1.000 & 1.000 \\
\hline \multirow{4}{*}{3} & Gravel pad & $\$ 135,270$ & $\$ 0$ & & $\$ 13,007$ & $\$ 135,270$ & 2.066 & 0.781 & & 75.036 & 0.500 & 0.750 & 0.500 & 0.500 \\
\hline & DURA-BASE from Composite Mat (buy) & $\$ 1,855,800$ & $\$ 1,484,640$ & & $\$ 35,688$ & $\$ 371,160$ & 1.033 & 0.984 & & 62.356 & 0.750 & 0.750 & 0.750 & 1.000 \\
\hline & DURA-BASE from Composite Mat (rent) & & & & & $\$ 100,800$ & 1.033 & 0.984 & & 62.356 & 0.750 & 0.750 & 0.750 & 1.000 \\
\hline & Aluminum modules + driven piles & $\$ 1,131,303$ & $\$ 905,042$ & & $\$ 21,756$ & $\$ 226,261$ & 0.005 & 0.989 & & 76.265 & 1.000 & 0.500 & 1.000 & 0.500 \\
\hline 4 & Rapid Rig & & & $\$ 14,000$ & $\$ 14,000$ & $\$ 168,000$ & & 0.986 & & 60.016 & 1.000 & 0.500 & 1.000 & 1.000 \\
\hline \multirow{3}{*}{5} & Internal combustion engine & & & $\$ 4,500$ & $\$ 4,500$ & $\$ 46,800$ & & 0.345 & & 107.691 & 0.500 & 1.000 & 0.500 & 0.750 \\
\hline & \multicolumn{2}{|l|}{ Internal combustion engine $\mathrm{w} / \mathrm{SCR}, \mathrm{w} / \mathrm{noise}$ suppressor } & & $\$ 6,670$ & $\$ 6,670$ & $\$ 69,363$ & & 0.578 & & 86.153 & 0.750 & 0.750 & 0.750 & 0.750 \\
\hline & \multicolumn{2}{|l|}{ Lean-burn natural gas engines $\mathrm{w} /$ noise suppressor } & & $\$ 7,035$ & $\$ 7,035$ & $\$ 73,168$ & & 0.909 & & 86.153 & 1.000 & 0.500 & 1.000 & 0.750 \\
\hline \multirow{3}{*}{6} & Conventional diesel & & & $\$ 4,320$ & $\$ 4,320$ & $\$ 43,848$ & & & & & 0.500 & 1.000 & 0.500 & 0.500 \\
\hline & Low sulphur diesel & & & $\$ 4,536$ & $\$ 4,536$ & $\$ 46,040$ & & & & & 0.750 & 0.750 & 1.000 & 0.750 \\
\hline & Natural gas & & & $\$ 2,700$ & $\$ 2,700$ & $\$ 27,405$ & & & & & 1.000 & 0.500 & 1.000 & 0.750 \\
\hline 7 & Electric power from grid $(10 \%)$ & & & $\$ 384$ & $\$ 384$ & $\$ 3,994$ & 0.000 & 1.000 & & 0.000 & 0.500 & 1.000 & 1.000 & 1.000 \\
\hline 8 & Flywheels & $\$ 150,000$ & $\$ 120,000$ & & $\$ 2,885$ & $\$ 30,000$ & 0.000 & & & & 0.500 & 1.000 & 1.000 & 0.750 \\
\hline 9 & Underbalanced drilling w/noise suppressor & & & $\$ 20,500$ & $\$ 20,500$ & $\$ 202,950$ & & & & 96.250 & 0.750 & 0.750 & 0.750 & 0.750 \\
\hline 10 & Water-based muds & & & & & $\$ 47,940$ & & & & & 1.000 & 1.000 & 1.000 & 1.000 \\
\hline \multirow{2}{*}{11} & Lined reserve pit + solid control equip.* & & & $\$ 2,000$ & $\$ 2,000$ & $\$ 19,800$ & 0.037 & & 0.500 & & 0.750 & 0.750 & 0.750 & 0.500 \\
\hline & Closed loop + containers + solid control equip. ${ }^{*}$ & & & $\$ 3,000$ & $\$ 3,000$ & $\$ 29,700$ & 0.000 & & 1.000 & & 1.000 & 0.500 & 1.000 & 0.750 \\
\hline \multirow{2}{*}{12} & Cuttings injection & & & $\$ 5,000$ & $\$ 5,000$ & $\$ 49,500$ & & & 1.000 & & 1.000 & 0.500 & 1.000 & 0.750 \\
\hline & Chemical fixation and solidification (CFS) & & & & & $\$ 61,710$ & & & 0.250 & & 0.750 & 0.750 & 1.000 & 0.500 \\
\hline 13 & N/A & & & & & & & & & & & & & \\
\hline
\end{tabular}

[When 'LOC250 (CWD)' is selected]

\begin{tabular}{|c|c|c|c|c|c|c|c|c|c|c|c|c|c|c|}
\hline \multirow{2}{*}{$\begin{array}{l}\text { Sub- } \\
\text { sets }\end{array}$} & \multirow[b]{2}{*}{ Technologies } & \multirow[b]{2}{*}{ Buy(\$) } & \multirow{2}{*}{$\begin{array}{c}\text { Resale } \\
\text { Value (\$) }\end{array}$} & \multirow{2}{*}{$\begin{array}{c}\text { Rent } / \text { day } \\
\text { (\$) }\end{array}$} & \multirow{2}{*}{\begin{tabular}{|c|} 
Daily \\
Rate (\$)
\end{tabular}} & \multirow{2}{*}{$\begin{array}{c}\text { Total cost } \\
\text { (\$) }\end{array}$} & \multirow{2}{*}{$\begin{array}{c}\text { Ecological } \\
\text { Footprint } \\
\text { (Acres) }\end{array}$} & \multicolumn{3}{|c|}{ Emissions } & \multicolumn{3}{|c|}{ Perceptions } & \multirow{2}{*}{$\begin{array}{l}\text { Safety } \\
\text { Value }\end{array}$} \\
\hline & & & & & & & & Air & $\begin{array}{l}\text { Solid\& } \\
\text { Liquid }\end{array}$ & \begin{tabular}{|l|} 
Noise \\
(TWA)
\end{tabular} & Gov. & Ind. & Public & \\
\hline 1 & \multicolumn{2}{|c|}{ Low sulphur diesel truck w/tier III engine, w/noise suppressor } & & & & & & & & & 1.000 & 0.500 & 1.000 & 0.750 \\
\hline \multirow{3}{*}{2} & Gravel roads & $\$ 198,396$ & $\$ 0$ & & $\$ 19,840$ & $\$ 198,396$ & 3.030 & 0.679 & & 77.250 & 0.500 & 0.750 & 0.500 & 0.500 \\
\hline & DURA-BASE from Composite Mat (buy) & $\$ 2,721,840$ & $\$ 2,177,472$ & & $\$ 54,437$ & $\$ 544,368$ & 1.515 & 0.976 & & 64.696 & 1.000 & 0.500 & 1.000 & 1.000 \\
\hline & DURA-BASE from Composite Mat (rent) & & & & & $\$ 147,840$ & 1.515 & 0.976 & & 64.696 & 1.000 & 0.500 & 1.000 & 1.000 \\
\hline \multirow{4}{*}{3} & Gravel pad & $\$ 135,270$ & $\$ 0$ & & $\$ 13,527$ & $\$ 135,270$ & 2.066 & 0.781 & & 75.036 & 0.500 & 0.750 & 0.500 & 0.500 \\
\hline & DURA-BASE from Composite Mat (buy) & $\$ 1,855,800$ & $\$ 1,484,640$ & & $\$ 37,116$ & $\$ 371,160$ & 1.033 & 0.984 & & 62.356 & 0.750 & 0.750 & 0.750 & 1.000 \\
\hline & DURA-BASE from Composite Mat (rent) & & & & & $\$ 100,800$ & 1.033 & 0.984 & & 62.356 & 0.750 & 0.750 & 0.750 & 1.000 \\
\hline & Aluminum modules + driven piles & $\$ 1,131,303$ & $\$ 905,042$ & & $\$ 22,626$ & $\$ 226,261$ & 0.005 & 0.989 & & 76.265 & 1.000 & 0.500 & 1.000 & 0.500 \\
\hline 4 & LOC250 (CWD) & & & $\$ 15,000$ & $\$ 15,000$ & $\$ 173,800$ & & 0.985 & & 60.366 & 1.000 & 0.500 & 1.000 & 1.000 \\
\hline \multirow{3}{*}{5} & Internal combustion engine & & & $\$ 3,500$ & $\$ 3,500$ & $\$ 35,000$ & & 0.537 & & 104.678 & 0.500 & 1.000 & 0.500 & 0.750 \\
\hline & \multicolumn{2}{|l|}{ Internal combustion engine $\mathrm{w} / \mathrm{SCR}, \mathrm{w} /$ noise suppressor } & & $\$ 5,187$ & $\$ 5,187$ & $\$ 51,874$ & & 0.701 & & 83.742 & 0.750 & 0.750 & 0.750 & 0.750 \\
\hline & \multicolumn{2}{|l|}{ Lean-burn natural gas engines w/noise suppressor } & & $\$ 5,472$ & $\$ 5,472$ & $\$ 54,720$ & & 0.936 & & 83.742 & 1.000 & 0.500 & 1.000 & 0.750 \\
\hline \multirow{3}{*}{6} & Conventional diesel & & & $\$ 3,360$ & $\$ 3,360$ & $\$ 31,920$ & & & & & 0.500 & 1.000 & 0.500 & 0.500 \\
\hline & Low sulphur diesel & & & $\$ 3,528$ & $\$ 3,528$ & $\$ 33,516$ & & & & & 0.750 & 0.750 & 1.000 & 0.750 \\
\hline & Natural gas & & & $\$ 2,100$ & $\$ 2,100$ & $\$ 19,950$ & & & & & 1.000 & 0.500 & 1.000 & 0.750 \\
\hline 7 & Electric power from grid $(30 \%)$ & & & $\$ 1,152$ & $\$ 1,152$ & $\$ 11,520$ & 0.000 & 1.000 & & 0.000 & 0.500 & 1.000 & 1.000 & 1.000 \\
\hline 8 & Flywheels & $\$ 450,000$ & $\$ 360,000$ & & $\$ 9,000$ & $\$ 90,000$ & 0.000 & & & & 0.500 & 1.000 & 1.000 & 0.750 \\
\hline 9 & Managed pressure drilling $\mathrm{w} / \mathrm{noise}$ suppressor & & & $\$ 21,500$ & $\$ 21,500$ & $\$ 193,500$ & & & & 94.100 & 0.750 & 0.750 & 1.000 & 1.000 \\
\hline 10 & Water-based muds & & & & & $\$ 47,940$ & & & & & 1.000 & 1.000 & 1.000 & 1.000 \\
\hline \multirow{2}{*}{11} & Lined reserve pit + solid control equip.* & & & $\$ 2,000$ & $\$ 2,000$ & $\$ 18,000$ & 0.037 & & 0.500 & & 0.750 & 0.750 & 0.750 & 0.500 \\
\hline & Closed loop + containers + solid control equip. ${ }^{*}$ & & & $\$ 3,000$ & $\$ 3,000$ & $\$ 27,000$ & 0.000 & & 1.000 & & 1.000 & 0.500 & 1.000 & 0.750 \\
\hline \multirow{2}{*}{12} & Cuttings injection & & & $\$ 5,000$ & $\$ 5,000$ & $\$ 45,000$ & & & 1.000 & & 1.000 & 0.500 & 1.000 & 0.750 \\
\hline & Chemical fixation and solidification (CFS) & & & & & $\$ 61,710$ & & & 0.250 & & 0.750 & 0.750 & 1.000 & 0.500 \\
\hline 13 & N/A & & & & & & & & & & & & & \\
\hline
\end{tabular}


[Input values and single-attribute utility scores of SET 1]

\begin{tabular}{|c|c|c|c|c|c|c|c|c|c|}
\hline \multirow{2}{*}{$\begin{array}{l}\text { Selected Technologies } \\
\text { in Each Subset }\end{array}$} & \multirow{2}{*}{$\begin{array}{c}\text { Total Cost } \\
(\$)\end{array}$} & \multirow{2}{*}{$\begin{array}{c}\text { Ecological } \\
\text { Footprint } \\
\text { (Acres) }\end{array}$} & \multicolumn{3}{|c|}{ Emissions } & \multicolumn{3}{|c|}{ Perceptions } & \multirow{2}{*}{$\begin{array}{l}\text { Safety } \\
\text { Value }\end{array}$} \\
\hline & & & Air & $\begin{array}{l}\text { Solid\& } \\
\text { Liquid }\end{array}$ & $\begin{array}{l}\text { Noise } \\
\text { (TWA) }\end{array}$ & Gov. & Ind. & Public & \\
\hline $\begin{array}{l}\text { (1) Transportation: Low sulphur diesel truck w/tier III engine, } \\
\text { w/noise suppressor }\end{array}$ & & & & & & 1.000 & 0.500 & 1.000 & 0.750 \\
\hline (2) Road construction: DURA-BASE from Composite Mat (rent) & $\$ 147,840$ & 1.515 & 0.976 & & 64.696 & 1.000 & 0.500 & 1.000 & 1.000 \\
\hline (3) Site preparation: Aluminum modules + driven piles & $\$ 226,261$ & 0.005 & 0.989 & & 76.265 & 1.000 & 0.500 & 1.000 & 0.500 \\
\hline (4) Rig type: LOC250 (CWD) & $\$ 173,800$ & & 0.985 & & 60.366 & 1.000 & 0.500 & 1.000 & 1.000 \\
\hline $\begin{array}{l}\text { (5) Rig power (Conventional): Lean-burn natural gas engines } \\
\text { w/noise suppressor }\end{array}$ & $\$ 70,354$ & & 0.918 & & 85.603 & 1.000 & 0.500 & 1.000 & 0.750 \\
\hline (6) Fuel type: Natural gas & $\$ 25,650$ & & & & & 1.000 & 0.500 & 1.000 & 0.750 \\
\hline (7) Rig power (Unconventional): Electric power from grid (10 \%) & $\$ 3,840$ & 0.000 & 1.000 & & 0.000 & 0.500 & 1.000 & 1.000 & 1.000 \\
\hline (8) Energy storage: Flywheels & $\$ 30,000$ & 0.000 & & & & 0.500 & 1.000 & 1.000 & 0.750 \\
\hline (9) Drilling tech.: Managed pressure drilling w/noise suppressor & $\$ 193,500$ & & & & 94.100 & 0.750 & 0.750 & 1.000 & 1.000 \\
\hline (10) Fluid type: Water-based muds & $\$ 47,940$ & & & & & 1.000 & 1.000 & 1.000 & 1.000 \\
\hline (11) Waste mgmt.: Closed loop + containers + solid control equip.* & $\$ 27,000$ & 0.000 & & 1.000 & & 1.000 & 0.500 & 1.000 & 0.750 \\
\hline (12) Cuttings mgmt:: Cuttings injection & $\$ 45,000$ & & & 1.000 & & 1.000 & 0.500 & 1.000 & 0.750 \\
\hline \multicolumn{10}{|l|}{ (13) Noise reduction: N/A } \\
\hline Overall Attribute Scores ( $\Sigma$ or minimum value) & $\$ 991,184$ & 1.520 & 4.868 & 2.000 & 381.030 & 0.500 & 0.500 & 1.000 & 0.500 \\
\hline Single Attribute Utility Values & 0.811 & 1.000 & 0.989 & 1.000 & 0.988 & 0.500 & 0.500 & 1.000 & 0.500 \\
\hline
\end{tabular}

[Input values and single-attribute utility scores of SET 2]

\begin{tabular}{|c|c|c|c|c|c|c|c|c|c|}
\hline \multirow{2}{*}{$\begin{array}{l}\text { Selected Technologies } \\
\text { in Each Subset }\end{array}$} & \multirow{2}{*}{$\begin{array}{l}\text { Total Cost } \\
(\$)\end{array}$} & \multirow{2}{*}{$\begin{array}{c}\text { Ecological } \\
\text { Footprint } \\
\text { (Acres) }\end{array}$} & \multicolumn{3}{|c|}{ Emissions } & \multicolumn{3}{|c|}{ Perceptions } & \multirow{2}{*}{$\begin{array}{l}\text { Safety } \\
\text { Value }\end{array}$} \\
\hline & & & Air & $\begin{array}{l}\text { Solid\& } \\
\text { Liquid }\end{array}$ & $\begin{array}{l}\text { Noise } \\
\text { (TWA) }\end{array}$ & Gov. & Ind. & Public & \\
\hline $\begin{array}{l}\text { (1) Transportation: Low sulphur diesel truck w/tier III engine, } \\
\text { w/noise suppressor }\end{array}$ & & & & & & 1.000 & 0.500 & 1.000 & 0.750 \\
\hline (2) Road construction: DURA-BASE from Composite Mat (rent) & $\$ 147,840$ & 1.515 & 0.976 & & 64.696 & 1.000 & 0.500 & 1.000 & 1.000 \\
\hline (3) Site preparation: DURA-BASE from Composite Mat (rent) & $\$ 100,800$ & 1.033 & 0.984 & & 62.356 & 0.750 & 0.750 & 0.750 & 1.000 \\
\hline (4) Rig type: LOC250 (CWD) & $\$ 173,800$ & & 0.985 & & 60.366 & 1.000 & 0.500 & 1.000 & 1.000 \\
\hline $\begin{array}{l}\text { (5) Rig power (Conventional): Lean-burn natural gas engines } \\
\text { w/noise suppressor }\end{array}$ & $\$ 70,354$ & & 0.918 & & 85.603 & 1.000 & 0.500 & 1.000 & 0.750 \\
\hline (6) Fuel type: Natural gas & $\$ 25,650$ & & & & & 1.000 & 0.500 & 1.000 & 0.750 \\
\hline (7) Rig power (Unconventional): Electric power from grid (10\%) & $\$ 3,840$ & 0.000 & 1.000 & & 0.000 & 0.500 & 1.000 & 1.000 & 1.000 \\
\hline (8) Energy storage: Flywheels & $\$ 30,000$ & 0.000 & & & & 0.500 & 1.000 & 1.000 & 0.750 \\
\hline (9) Drilling tech.: Underbalanced drilling w/noise suppressor & $\$ 184,500$ & & & & 95.700 & 0.750 & 0.750 & 0.750 & 0.750 \\
\hline (10) Fluid type: Water-based muds & $\$ 47,940$ & & & & & 1.000 & 1.000 & 1.000 & 1.000 \\
\hline (11) Waste mgmt.: Closed loop + containers + solid control equip." & $\$ 27,000$ & 0.000 & & 1.000 & & 1.000 & 0.500 & 1.000 & 0.750 \\
\hline (12) Cuttings mgmt.: Cuttings injection & $\$ 45,000$ & & & 1.000 & & 1.000 & 0.500 & 1.000 & 0.750 \\
\hline \multicolumn{10}{|l|}{ (13) Noise reduction: N/A } \\
\hline Overall Attribute Scores ( $\Sigma$ or minimum value) & $\$ 856,724$ & 2.548 & 4.863 & 2.000 & 368.721 & 0.500 & 0.500 & 0.750 & 0.750 \\
\hline Single Attribute Utility Values & 0.931 & 0.764 & 0.986 & 1.000 & 0.998 & 0.500 & 0.500 & 0.750 & 0.750 \\
\hline
\end{tabular}


[Input values and single-attribute utility scores of SET 3]

\begin{tabular}{|c|c|c|c|c|c|c|c|c|c|}
\hline \multirow{2}{*}{$\begin{array}{l}\text { Selected Technologies } \\
\text { in Each Subset }\end{array}$} & \multirow{2}{*}{$\begin{array}{c}\text { Total Cost } \\
\text { (\$) }\end{array}$} & \multirow{2}{*}{$\begin{array}{c}\text { Ecological } \\
\text { Footprint } \\
\text { (Acres) }\end{array}$} & \multicolumn{3}{|c|}{ Emissions } & \multicolumn{3}{|c|}{ Perceptions } & \multirow{2}{*}{$\begin{array}{l}\text { Safety } \\
\text { Value }\end{array}$} \\
\hline & & & Air & $\begin{array}{l}\text { Solid\& } \\
\text { Liquid }\end{array}$ & $\begin{array}{l}\text { Noise } \\
\text { (TWA })\end{array}$ & Gov. & Ind. & Public & \\
\hline (1) Transportation: Coventional diesel truck & & & & & & 0.250 & 1.000 & 0.250 & 0.750 \\
\hline (2) Road construction: DURA-BASE from Composite Mat (rent) & $\$ 132,000$ & 1.515 & 0.964 & & 82.870 & 1.000 & 0.500 & 1.000 & 1.000 \\
\hline (3) Site preparation: DURA-BASE from Composite Mat (rent) & $\$ 90,000$ & 1.033 & 0.976 & & 79.945 & 0.750 & 0.750 & 0.750 & 1.000 \\
\hline (4) Rig type: LOC250 (CWD) & $\$ 167,000$ & & 0.977 & & 77.458 & 1.000 & 0.500 & 1.000 & 1.000 \\
\hline $\begin{array}{l}\text { (5) Rig power (Conventional): Lean-burn natural gas engines } \\
\text { w/noise suppressor }\end{array}$ & $\$ 78,171$ & & 0.908 & & 86.399 & 1.000 & 0.500 & 1.000 & 0.750 \\
\hline (6) Fuel type: Natural gas & $\$ 28,500$ & & & & & 1.000 & 0.500 & 1.000 & 0.750 \\
\hline (7) Rig power (Unconventional): N/A (0 \%) & $\$ 0$ & 0.000 & 1.000 & & 0.000 & 0.250 & 1.000 & 0.250 & 1.000 \\
\hline (8) Energy storage: N/A & $\$ 0$ & 0.000 & & & & 0.250 & 1.000 & 0.250 & 1.000 \\
\hline (9) Drilling tech.: Underbalanced drilling w/noise suppressor & $\$ 184,500$ & & & & 95.700 & 0.750 & 0.750 & 0.750 & 0.750 \\
\hline (10) Fluid type: Water-based muds & $\$ 47,940$ & & & & & 1.000 & 1.000 & 1.000 & 1.000 \\
\hline (11) Waste mgmt.: Closed loop + containers + solid control equip.* & $\$ 27,000$ & 0.000 & & 1.000 & & 1.000 & 0.500 & 1.000 & 0.750 \\
\hline (12) Cuttings mgmt:: Cuttings injection & $\$ 45,000$ & & & 1.000 & & 1.000 & 0.500 & 1.000 & 0.750 \\
\hline \multicolumn{10}{|l|}{ (13) Noise reduction: N/A } \\
\hline Overall Attribute Scores ( $\Sigma$ or minimum value) & $\$ 800,111$ & 2.548 & 4.825 & 2.000 & 422.371 & 0.250 & 0.500 & 0.250 & 0.750 \\
\hline Single Attribute Utility Values & 0.982 & 0.764 & 0.962 & 1.000 & 0.911 & 0.250 & 0.500 & 0.250 & 0.750 \\
\hline
\end{tabular}

[Input values and single-attribute utility scores of SET 4]

\begin{tabular}{|c|c|c|c|c|c|c|c|c|c|}
\hline \multirow{2}{*}{$\begin{array}{l}\text { Selected Technologies } \\
\text { in Each Subset }\end{array}$} & \multirow{2}{*}{$\begin{array}{c}\text { Total Cost } \\
(\$)\end{array}$} & \multirow{2}{*}{$\begin{array}{c}\text { Ecological } \\
\text { Footprint } \\
\text { (Acres) }\end{array}$} & \multicolumn{3}{|c|}{ Emissions } & \multicolumn{3}{|c|}{ Perceptions } & \multirow{2}{*}{$\begin{array}{l}\text { Safety } \\
\text { Value }\end{array}$} \\
\hline & & & Air & $\begin{array}{l}\text { Solid\& } \\
\text { Liquid }\end{array}$ & $\begin{array}{l}\text { Noise } \\
\text { (TWA) }\end{array}$ & Gov. & Ind. & Public & \\
\hline $\begin{array}{l}\text { (1) Transportation: Low sulphur diesel truck w/tier III engine, } \\
\text { w/noise suppressor }\end{array}$ & & & & & & 1.000 & 0.500 & 1.000 & 0.750 \\
\hline (2) Road construction: DURA-BASE from Composite Mat (rent) & $\$ 147,840$ & 1.515 & 0.976 & & 64.696 & 1.000 & 0.500 & 1.000 & 1.000 \\
\hline (3) Site preparation: Aluminum modules + driven piles & $\$ 226,261$ & 0.005 & 0.989 & & 76.265 & 1.000 & 0.500 & 1.000 & 0.500 \\
\hline (4) Rig type: LOC250 (CWD) & $\$ 173,800$ & & 0.985 & & 60.366 & 1.000 & 0.500 & 1.000 & 1.000 \\
\hline $\begin{array}{l}\text { (5) Rig power (Conventional): Lean-burn natural gas engines } \\
\text { w/noise suppressor }\end{array}$ & $\$ 54,720$ & & 0.936 & & 83.742 & 1.000 & 0.500 & 1.000 & 0.750 \\
\hline (6) Fuel type: Natural gas & $\$ 19,950$ & & & & & 1.000 & 0.500 & 1.000 & 0.750 \\
\hline (7) Rig power (Unconventional): Electric power from grid (30 \%) & $\$ 11,520$ & 0.000 & 1.000 & & 0.000 & 0.500 & 1.000 & 1.000 & 1.000 \\
\hline (8) Energy storage: Flywheels & $\$ 90,000$ & 0.000 & & & & 0.500 & 1.000 & 1.000 & 0.750 \\
\hline (9) Drilling tech.: Managed pressure drilling w/noise suppressor & $\$ 193,500$ & & & & 94.100 & 0.750 & 0.750 & 1.000 & 1.000 \\
\hline (10) Fluid type: Water-based muds & $\$ 47,940$ & & & & & 1.000 & 1.000 & 1.000 & 1.000 \\
\hline (11) Waste mgmt.: Closed loop + containers + solid control equip.* & $\$ 27,000$ & 0.000 & & 1.000 & & 1.000 & 0.500 & 1.000 & 0.750 \\
\hline (12) Cuttings mgmt:: Cuttings injection & $\$ 45,000$ & & & 1.000 & & 1.000 & 0.500 & 1.000 & 0.750 \\
\hline \multicolumn{10}{|l|}{ (13) Noise reduction: N/A } \\
\hline Overall Attribute Scores ( $\Sigma$ or minimum value) & $\$ 1,037,530$ & 1.520 & 4.886 & 2.000 & 379.169 & 0.500 & 0.500 & 1.000 & 0.500 \\
\hline Single Attribute Utility Values & 0.769 & 1.000 & 1.000 & 1.000 & 0.989 & 0.500 & 0.500 & 1.000 & 0.500 \\
\hline
\end{tabular}


[Input values and single-attribute utility scores of SET 5]

\begin{tabular}{|c|c|c|c|c|c|c|c|c|c|}
\hline \multirow{2}{*}{$\begin{array}{l}\text { Selected Technologies } \\
\text { in Each Subset }\end{array}$} & \multirow{2}{*}{$\begin{array}{c}\text { Total Cost } \\
(\$)\end{array}$} & \multirow{2}{*}{$\begin{array}{c}\text { Ecological } \\
\text { Footprint } \\
\text { (Acres) }\end{array}$} & \multicolumn{3}{|c|}{ Emissions } & \multicolumn{3}{|c|}{ Perceptions } & \multirow{2}{*}{$\begin{array}{l}\text { Safety } \\
\text { Value }\end{array}$} \\
\hline & & & Air & $\begin{array}{l}\text { Solid\& } \\
\text { Liquid }\end{array}$ & $\begin{array}{l}\text { Noise } \\
\text { (TWA) }\end{array}$ & Gov. & Ind. & Public & \\
\hline (1) Transportation: Coventional diesel truck & & & & & & 0.250 & 1.000 & 0.250 & 0.750 \\
\hline (2) Road construction: DURA-BASE from Composite Mat (rent) & $\$ 132,000$ & 1.515 & 0.964 & & 82.870 & 1.000 & 0.500 & 1.000 & 1.000 \\
\hline (3) Site preparation: DURA-BASE from Composite Mat (rent) & $\$ 90,000$ & 1.033 & 0.976 & & 79.945 & 0.750 & 0.750 & 0.750 & 1.000 \\
\hline (4) Rig type: LOC250 (CWD) & $\$ 167,000$ & & 0.977 & & 77.458 & 1.000 & 0.500 & 1.000 & 1.000 \\
\hline (5) Rig power (Conventional): Internal combustion engine & $\$ 50,000$ & & 0.338 & & 107.998 & 0.500 & 1.000 & 0.500 & 0.750 \\
\hline (6) Fuel type: Low sulphur diesel & $\$ 47,880$ & & & & & 0.750 & 0.750 & 1.000 & 0.750 \\
\hline (7) Rig power (Unconventional): N/A ( $0 \%)$ & $\$ 0$ & 0.000 & 1.000 & & 0.000 & 0.250 & 1.000 & 0.250 & 1.000 \\
\hline (8) Energy storage: N/A & $\$ 0$ & 0.000 & & & & 0.250 & 1.000 & 0.250 & 1.000 \\
\hline (9) Drilling tech.: Underbalanced drilling w/noise suppressor & $\$ 184,500$ & & & & 95.700 & 0.750 & 0.750 & 0.750 & 0.750 \\
\hline (10) Fluid type: Water-based muds & $\$ 47,940$ & & & & & 1.000 & 1.000 & 1.000 & 1.000 \\
\hline (11) Waste mgmt.: Closed loop + containers + solid control equip.* & $\$ 27,000$ & 0.000 & & 1.000 & & 1.000 & 0.500 & 1.000 & 0.750 \\
\hline (12) Cuttings mgmt.: Cuttings injection & $\$ 45,000$ & & & 1.000 & & 1.000 & 0.500 & 1.000 & 0.750 \\
\hline \multicolumn{10}{|l|}{ (13) Noise reduction: N/A } \\
\hline Overall Attribute Scores ( $\Sigma$ or minimum value) & $\$ 791,320$ & 2.548 & 4.254 & 2.000 & 443.971 & 0.250 & 0.500 & 0.250 & 0.750 \\
\hline Single Attribute Utility Values & 0.990 & 0.764 & 0.613 & 1.000 & 0.820 & 0.250 & 0.500 & 0.250 & 0.750 \\
\hline
\end{tabular}

[Input values and single-attribute utility scores of SET 6]

\begin{tabular}{|c|c|c|c|c|c|c|c|c|c|}
\hline \multirow{2}{*}{$\begin{array}{l}\text { Selected Technologies } \\
\text { in Each Subset }\end{array}$} & \multirow{2}{*}{$\begin{array}{c}\text { Total Cost } \\
(\$)\end{array}$} & \multirow{2}{*}{$\begin{array}{c}\text { Ecological } \\
\text { Footprint } \\
\text { (Acres) }\end{array}$} & \multicolumn{3}{|c|}{ Emissions } & \multicolumn{3}{|c|}{ Perceptions } & \multirow{2}{*}{$\begin{array}{l}\text { Safety } \\
\text { Value }\end{array}$} \\
\hline & & & Air & $\begin{array}{l}\text { Solid\& } \\
\text { Liquid }\end{array}$ & $\begin{array}{l}\text { Noise } \\
\text { (TWA) }\end{array}$ & Gov. & Ind. & Public & \\
\hline (1) Transportation: Coventional diesel truck & & & & & & 0.250 & 1.000 & 0.250 & 0.750 \\
\hline (2) Road construction: DURA-BASE from Composite Mat (rent) & $\$ 132,000$ & 1.515 & 0.964 & & 82.870 & 1.000 & 0.500 & 1.000 & 1.000 \\
\hline (3) Site preparation: DURA-BASE from Composite Mat (rent) & $\$ 90,000$ & 1.033 & 0.976 & & 79.945 & 0.750 & 0.750 & 0.750 & 1.000 \\
\hline (4) Rig type: LOC250 (CWD) & $\$ 167,000$ & & 0.977 & & 77.458 & 1.000 & 0.500 & 1.000 & 1.000 \\
\hline (5) Rig power (Conventional): Internal combustion engine & $\$ 50,000$ & & 0.338 & & 107.998 & 0.500 & 1.000 & 0.500 & 0.750 \\
\hline (6) Fuel type: Conventional diesel & $\$ 45,600$ & & & & & 0.500 & 1.000 & 0.500 & 0.500 \\
\hline (7) Rig power (Unconventional): N/A (0\%) & $\$ 0$ & 0.000 & 1.000 & & 0.000 & 0.250 & 1.000 & 0.250 & 1.000 \\
\hline (8) Energy storage: N/A & $\$ 0$ & 0.000 & & & & 0.250 & 1.000 & 0.250 & 1.000 \\
\hline (9) Drilling tech.: Underbalanced drilling w/noise suppressor & $\$ 184,500$ & & & & 95.700 & 0.750 & 0.750 & 0.750 & 0.750 \\
\hline (10) Fluid type: Water-based muds & $\$ 47,940$ & & & & & 1.000 & 1.000 & 1.000 & 1.000 \\
\hline (11) Waste mgmt.: Lined reserve pit + solid control equip.* & $\$ 18,000$ & 0.037 & & 0.500 & & 0.750 & 0.750 & 0.750 & 0.500 \\
\hline (12) Cuttings mgmt.: Cuttings injection & $\$ 45,000$ & & & 1.000 & & 1.000 & 0.500 & 1.000 & 0.750 \\
\hline \multicolumn{10}{|l|}{ (13) Noise reduction: N/A } \\
\hline Overall Attribute Scores ( $\Sigma$ or minimum value) & $\$ 780,040$ & 2.585 & 4.254 & 1.500 & 443.971 & 0.250 & 0.500 & 0.250 & 0.500 \\
\hline Single Attribute Utility Values & 1.000 & 0.756 & 0.613 & 0.600 & 0.820 & 0.250 & 0.500 & 0.250 & 0.500 \\
\hline
\end{tabular}


The knapsack optimization model for the EFD technology selection problem with nine attributes is given as follows:

$$
\operatorname{Maximize} U=\sum_{\mathrm{j}=1}^{\mathrm{J}} \mathrm{y}_{\mathrm{j}}\left[\sum_{\mathrm{i}=1}^{9} \mathrm{k}_{\mathrm{i}} \mathrm{u}_{\mathrm{i}}\left(\mathrm{X}_{\mathrm{ij}}\right)\right]
$$

where $\mathrm{j}$ is the index for systems, $\mathrm{J}$ is the number of possible systems, $\mathrm{i}$ is the index for the attributes, $\mathrm{k}_{\mathrm{i}}$ is the weight assigned to the $\mathrm{i}^{\text {th }}$ attribute ( $\mathrm{k}$ must sum to 1 ), $\mathrm{X}_{\mathrm{ij}}$ is the overall score of the $\mathrm{j}^{\text {th }}$ system on the $\mathrm{i}^{\text {th }}$ attribute, $\mathrm{u}_{\mathrm{i}}\left(\mathrm{X}_{\mathrm{ij}}\right)$ is the single-attribute utility value for system $\mathrm{j}$ on attribute $\mathrm{i}$, scaled from 0 to 1 , and $\mathrm{y}_{\mathrm{j}}$ is a binary decision variable that is one if system $\mathrm{j}$ is selected and zero if it is not. In order to calculate the overall score of a system on the $\mathrm{i}^{\text {th }}$ attribute $\left(\mathrm{X}_{\mathrm{i}}\right)$, Eq. (5-1), Eq. (5-2), and Eq. (5-3) should be considered. The other constraint required to consider for this optimization problem is:

$$
\sum_{j=1}^{J} y_{j}=1
$$

where $\mathrm{y}_{\mathrm{j}}$ is a binary decision variable. 


\section{APPENDIX C}

\section{QUESTIONNAIRES ABOUT THE WEB-BASED DECISION OPTIMIZATION TOOL [VERSION 1.1]}


1. What do you think the biggest advantage of using the Web-based decision optimization application is?

- Well guided for selecting the options

- It optimized the data for us

- It allows you to consider impacts based on factors other than cost-like environmental effects

- It allows you to weigh the options

- You can weigh the options

- Generate optimized values based on weight factor we want to assign

- Convenience

- Brings out the best combination scenario for any particulate area

- We managed to use the system efficiently and the system was able to optimize our data

- It relates the perception value to a dollar amount

- Each section is very systematic

- Easy to input $\rightarrow$ location, $\rightarrow$ multiple users

- We can keep the video as note and choose the part that we need to review

- Easy, handy, and comprehensive

- Eases the calculation

- Making the decision simple

- This will be helping to organize the input and output process of the information

- Pulling data from multiple sources, and consolidation of sources

- Time saving

- Optimizing function. Easy, quick and efficient

- Give a good idea about technologies and methods for different areas

- Ready made cost and perception

- Being impacts based on factor and not only upon cost/ environment

- It lets you grade different attractive using weighting factors appropriate for each criterion. It makes it easy to evaluate alternatives since it outputs the best combination

- There is a consistent rubric that forces every group to consider the same factors

- Makes the optimization easier

- Does weighting technique extremely well. Very easy to use

- Ready made cost and perception

2. Do you think the application is ease to use? If no, please explain what the most difficult part of using the application is.

- Yes, the options were elaborate and therefore selecting was not cumbersome

- Yes, it's fairly easy to use and understand with basic knowledge of software and optimization principles

- No, setting up the system was complicated in the beginning. Making our own 
modifications was difficult

- I did not think it was super easy to use. It was difficult to set up

- Basic design of each action item might be not independent

- Fairly easy. Requires basic knowledge

- It's easy to use but some more explanation about how the perceptions should be chosen (i.e., 1 is what, 0 is what, what your answer represents?)

- Yes, but how to input the weighting factors was a bit confusing

- It was hard to start, but the example posted by Dr. Burnett was very helpful

- Application is easy enough to use but not flexible

- Result sheet

- Yes, but it is not practical

- Yes, but it does not have "Save as" option

- Some data were hard to obtain (e.g., perception from industry and public)

- No, it was very complex. It took us a lot of time to understand how to determine the points. It is also wage

- Most difficult part was to access the values to the emissions and what the public and government would think

- Modification not easy after set up

- The application is decently easy to use. The $\log$ in and user interface need to be improved

- Was easy but only if you explored it a little

- Yes, but if possible, develop a calculation for specific scenarios

3. Which part should be modified soon for the application?

- Maybe give a "Set-up" section with instructions

- Good instructions on what to do

- Better instructions on what to do and how to use it

- Some errors found in the results

- Put more description about scoring

- Not very familiar with system

- A little more instructions

- More instructions

- It would be good to simplify the process of inputting weighting factors.

- Perceptions are hard to estimate

- A percentage of power input, or power supplied needs to be added

- Can we show every step for the calculation?

- Minimum number of technologies analyzed should be less than 4

- The weighting point section

- The optimization matrix assumes that

- The factors and weight that are inserted are difficult and cumbersome to determine. Some fixed costs (standards) should be fixed 
- Units should be mad consistent

- The result summary page

- The optimization matrix assumes that power will be constant

- The outputs generated. I am not aware if there is a tutorial and manual on how to use it

4. Any other suggestion and comments for the application?

- If you do not want to select a technology from a certain Subset, just skip it. (Do not need to fill out " 0 " for that).

- Should be more specific details about perception factors

- Make it more secure. Want some permission thing

- Can be a powerful and very useful tool

- More examples would be helpful

- If you can add an option of populating the results in a pdf file

- Give options to input and remove parameters

- Neatly charted and user friendly

- Include columns for fixed cost and daily costs and give direction on how to prorate capitalized costs. Needs to be more finance based in order to find the present net values for the wells

- Matrix versatile

- Lab view type design

- Unique and informative source 


\section{VITA}

Name: $\quad$ Ok-Youn $\mathrm{Yu}$

Address: $\quad$ Zachry Department of Civil Engineering

Texas A\&M University

College Station, TX 77843-3136, USA

Email Address: tg0918@tamu.edu

Education

2006-2009 Ph.D., Civil Engineering, Texas A\&M University, TX.

2003-2005 M.S., Construction Management, Texas A\&M University, TX.

1992-1999 B.S., Civil Engineering, KonKuk University, Korea.

\section{Experience}

2006-2009 Texas A\&M University, College Station, TX.

Research Assistant, Zachry Department of Civil Engineering.

2004-2005 Texas A\&M University, College Station, TX.

Research Assistant, Department of Construction Science.

2000-2003 Daehan Consultants Co., Ltd., Seoul, Korea.

Bridge and Tunnel Design Engineer, Structural Department II.

1998-2000 Dongshin Engineering \& Development Co., Ltd., Seoul, Korea.

Structural Engineer, Structural Department. 